\title{
Analysis and Characterization of Nonlinear Distortion due to High Power Amplifiers in Wireless Communication Systems
}

by

\author{
Tarek Helaly \\ A thesis submitted to the Faculty of Graduate and Postdoctoral Affairs \\ in partial fulfillment of the requirements for the degree of \\ Doctor of Philosophy \\ in \\ Electrical and Computer Engineering \\ Ottawa-Carleton Institute for Electrical and Computer Engineering \\ Department of Systems and Computer Engineering \\ Carleton University \\ Ottawa, Ontario
}

CCopyright

2010, Tarek Helaly 
Library and Archives Canada

Published Heritage

Branch

395 Wellington Street Ottawa ON K1A ON4 Canada
Bibliothèque et

Archives Canada

Direction du

Patrimoine de l'édition

395, rue Wellington

Ottawa ON K1A 0N4

Canada
Your file Votre référence
ISBN: $978-0-494-70523-0$
Our file Notre référence
ISBN: $978-0-494-70523-0$

\section{NOTICE:}

The author has granted a nonexclusive license allowing Library and Archives Canada to reproduce, publish, archive, preserve, conserve, communicate to the public by telecommunication or on the Internet, loan, distribute and sell theses worldwide, for commercial or noncommercial purposes, in microform, paper, electronic and/or any other formats.

The author retains copyright ownership and moral rights in this thesis. Neither the thesis nor substantial extracts from it may be printed or otherwise reproduced without the author's permission.
AVIS:

L'auteur a accordé une licence non exclusive permettant à la Bibliothèque et Archives Canada de reproduire, publier, archiver, sauvegarder, conserver, transmettre au public par télécommunication ou par l'Internet, prêter, distribuer et vendre des thèses partout dans le monde, à des fins commerciales ou autres, sur support microforme, papier, électronique et/ou autres formats.

L'auteur conserve la propriété du droit d'auteur et des droits moraux qui protège cette thèse. $\mathrm{Ni}$ la thèse ni des extraits substantiels de celle-ci ne doivent être imprimés ou autrement reproduits sans son autorisation.
In compliance with the Canadian Privacy Act some supporting forms may have been removed from this thesis.

While these forms may be included in the document page count, their removal does not represent any loss of content from the thesis.
Conformément à la loi canadienne sur la protection de la vie privée, quelques formulaires secondaires ont été enlevés de cette thèse.

Bien que ces formulaires aient inclus dans la pagination, il n'y aura aucun contenu manquant.

\section{Canadä}


The undersigned recommend to the Faculty of Graduate and Postdoctoral Affairs acceptance of the thesis

\title{
Analysis and Characterization of Nonlinear Distortion due to High Power Amplifiers in Wireless Communication Systems
}

\author{
Submitted by \\ Tarek K. Helaly, B. Sc, M.Sc. \\ In partial fulfillment of the requirements for the degree of \\ Doctor of Philosophy \\ Thesis Co-supervisor \\ Professor M. S. El-Tanany \\ Thesis Co-supervisor \\ Professor R. M. Dansereau \\ External Examiner \\ Dr. L. Morant, Nanowave Technologies Inc.
}

Chair, Professor Howard Schwartz, Department of Systems and Computer Engineering

Carleton University

July 21,2010 


\section{ABSTRACT}

Code division multiple access (CDMA) and orthogonal frequency division multiplexing (OFDM) signaling schemes are widely used nowadays in wireless communication systems. They are particularly suited for the increased and continuous demands of more capacity, higher data rates, better quality of service, and robustness against multipath fading. Unfortunately, such signals exhibit large dynamic ranges, as a result, they are more susceptible to amplifier nonlinearity. The distortion caused by nonlinearities leads to bit error rate (BER) degradation and out-of-band spectral regrowth.

In this dissertation, the nonlinear distortion effects due to power amplifiers are analyzed. In particular, we are interested in analytically quantifying the signal's nonlinear behavior in relation to the amplifier characteristics. Through analyses, the characteristics of the input signal to the amplifier that contribute to BER degradation and spectral regrowth are explored. Based on such characteristics, a new measure characterizing the nonlinear distortion is formulated. With this formulation, an efficient system in presence of nonlinear distortion can be achieved by redirecting attention to the signal at the input of the amplifier. Specifically, we investigate methods to: (1) provide a potential estimate of the nonlinear distortion effects on the system performance and (2) minimize such effects by minimizing the signal characteristics contributing to performance degradation. 
Numerical experiments are performed to confirm the theoretical analyses and show the performance improvement of the developed measure in terms of an improved BER and reduced spectral regrowth. 


\section{ACKNOWLEDGMENTS}

Thanks GOD,

I would like to express my sincere appreciation to my supervisors Professor Mohamed El-Tanany and Professor Richard Dansereau. Mohamed and Richard provided me with the guidance, advice, wisdom, and support to pursue and successfully complete this work. I will continue to be influenced by their rigorous scholarship, clarity in thinking, and professional integrity.

I would like to thank Dr. Roshdy Hafez and Dr. Jim Wight of Carleton University and Dr. Abbas Yongacoglu of University of Ottawa for their feedbacks, constructive comments, and valuable suggestions on my research work.

I would like to express my thanks to Dr. Lincoln Morant of Nanowave Technologies Inc. for his willingness to serve as my external examiner.

Finally, I would like to thank my parents, my wife, and my children for their patience, understanding and support over all these years. All my achievements would not have been possible without their constant encouragement and support. 


\section{TABLE OF CONTENTS}

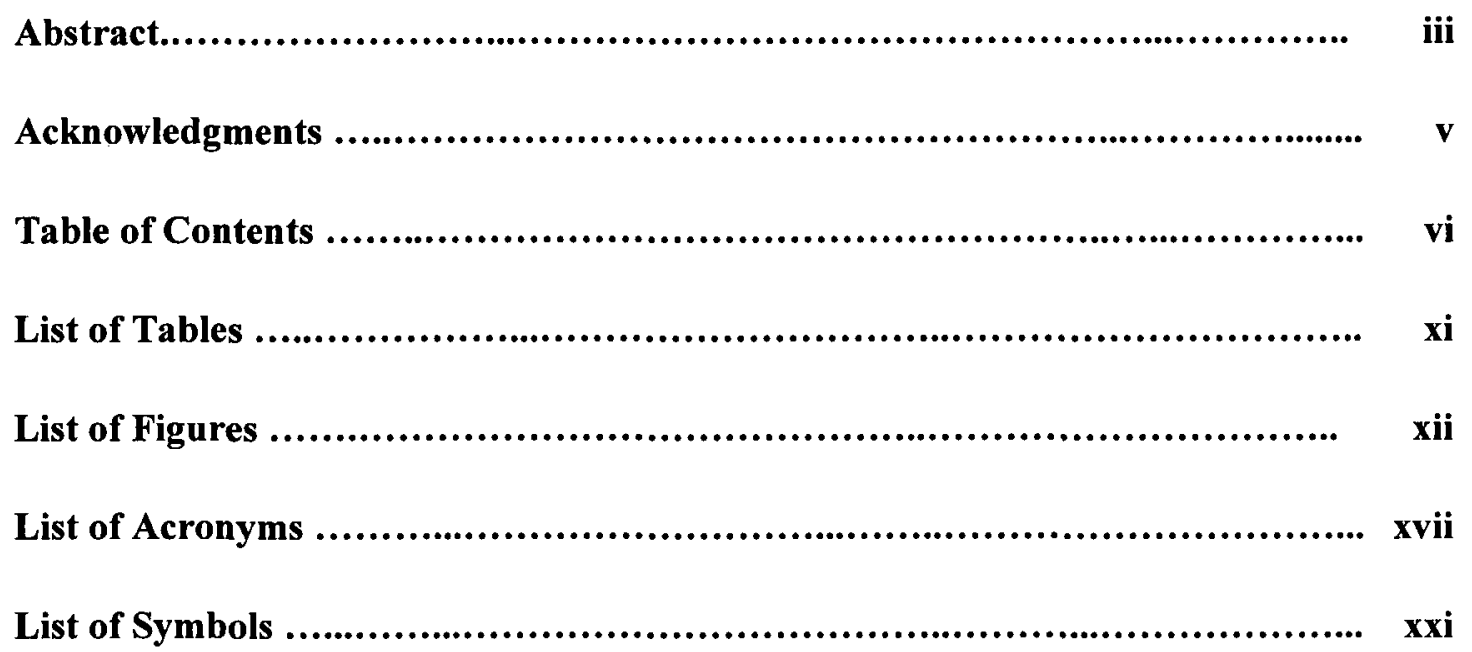

Chapter 1: Introduction ......................................................... 1

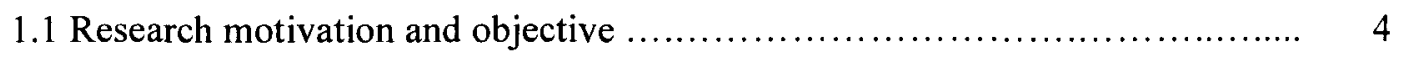

1.2 Considerations and research methodologies ............................... 5

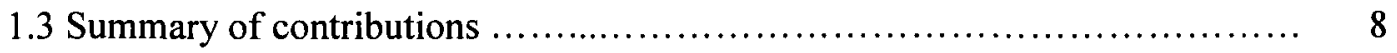

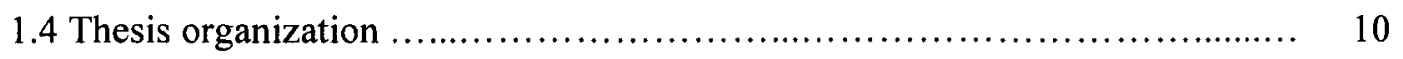

Chapter 2: Theoretical background ........................................... 13

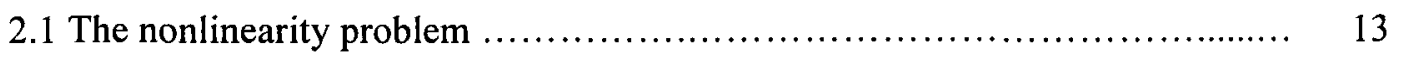


2.2 HPA characteristics.................................................. 16

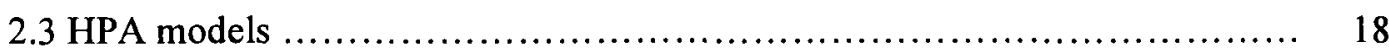

2.3.1 Saleh's model....................................................... 19

2.3.2 Rapp's model.................................................. $\quad 20$

2.3.3 Polynomial model $\ldots \ldots \ldots \ldots \ldots \ldots \ldots \ldots \ldots \ldots \ldots \ldots \ldots \ldots \ldots \ldots \ldots, 22$

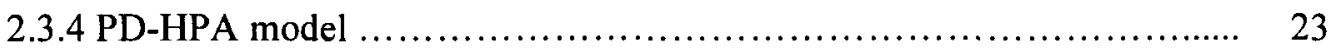

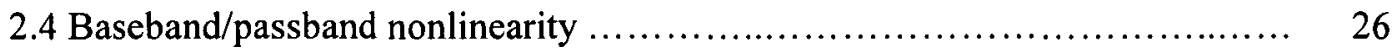

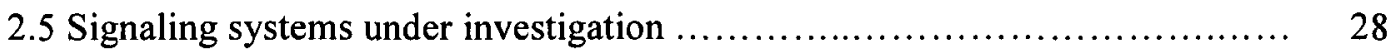

2.5.1 CDMA basics .................................................... 29

2.5.1.1 Transmitter system $\ldots \ldots \ldots \ldots \ldots \ldots \ldots \ldots \ldots \ldots \ldots \ldots \ldots \ldots \ldots . \quad 31$

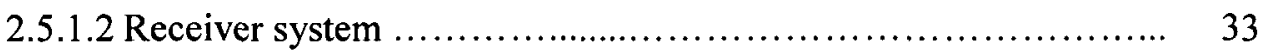

2.5.2 OFDM basics ................................................. 39

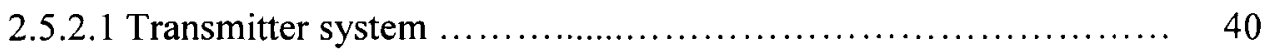

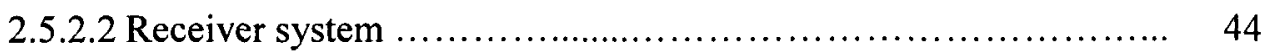

Chapter 3: Analysis of nonlinear distortion effects on BER performance........... 46

3.1 BER performance analysis for CDMA signals .......................... 48

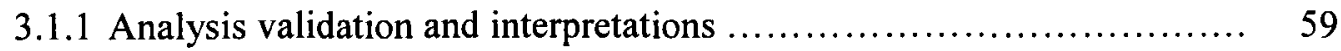

3.2 BER performance analysis for OFDM signals .......................... 66 
3.2.1 Analysis validation and interpretations ............................ 71

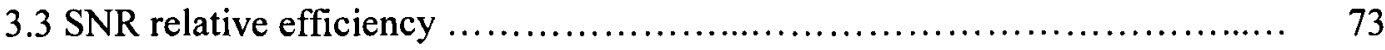

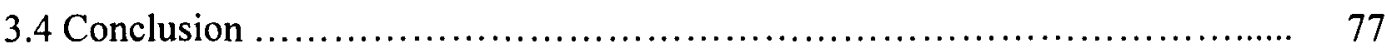

Chapter 4: Analysis of nonlinear distortion effects on spectral regrowth............. 78

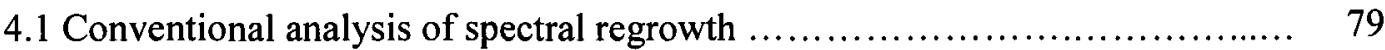

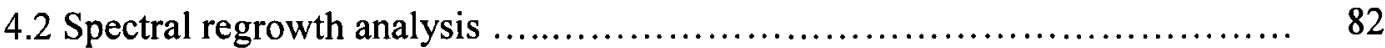

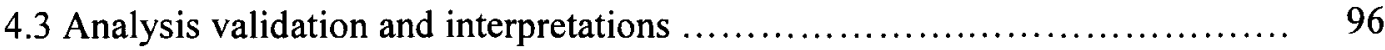

4.4 Special case: spectral regrowth reduction in CDMA systems with orthogonal

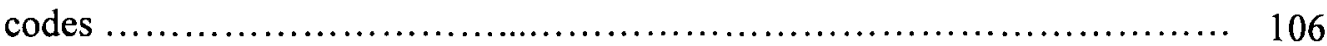

4.4.1 PSD analysis of CDMA signals with orthogonal codes............... 106

4.4.2 Walsh code properties $\ldots \ldots \ldots \ldots \ldots \ldots \ldots \ldots \ldots \ldots \ldots \ldots \ldots \ldots \ldots \ldots \ldots, 111$

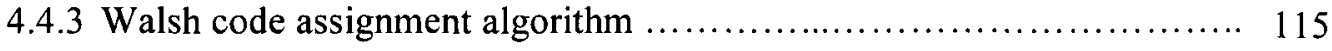

4.4.4 Performance assessment of the Walsh code assignment algorithm ........ 117

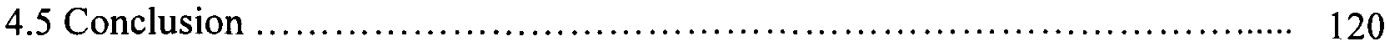

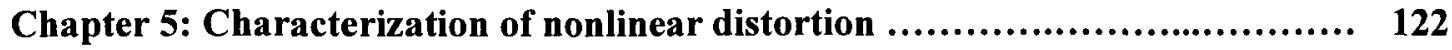

5.1 Existing measures for nonlinear distortion characterization.................... 122

5.2 Characterization of nonlinear distortion via nonlinearity severity measure...... 
Chapter 6: Performance assessment of the nonlinearity severity measure..........

6.1 Selected mapping (SLM) technique 135

6.1.1 SLM in CDMA 136

6.1.2 SLM in OFDM 144

6.2 Performance assessment of NLS in presence of PD-HPA 146

6.2.1 BER performance.

6.2.2 Spectral regrowth.

6.3 Performance assessment with different HPA models 161

6.3.1 Applicability of the NLS measure with other HPA models 162

6.4 Conclusions 167

Chapter 7: Conclusions and suggestions for future research 168

7.1 Conclusions 168

7.2 Suggestions for future research.

Appendix A: variance of the derivative of a random process with rectangular passband 
Appendix B: Joint probability density function of the envelope and its first derivative of complex Gaussian processes passband................ 177

References...................................................................... 180 


\section{LIST OF TABLES}

Table 3.1 BER performance of different modulation formats

Table 4.1 Signal characteristics for 8-user DS-CDMA signal representations......... 104

Table 4.2 Signal characteristics for 16-user DS-CDMA signal representations ..... 104

Table 4.3 Signal characteristics for 64-user DS-CDMA signal representations....... 104

Table 4.4 ACPR UPPER $_{\text {of }}$ of the three Walsh code selections.................................... 118

Table 5.1 Signal characteristics of representations R1, R2, and R3 ............. 125

Table 5.2 Signal characteristics of representations R1, R2, R3, R4, R5, and R6 ... 131

Table 6.1 CRR for the optimal and greedy algorithms ......................... 139

Table 6.2 ACPR for 32-user CDMA in presence of PD-HPA .................. 159

Table 6.3 ACPR for 16-user CDMA in presence of PD-HPA .................. 159

Table 6.4 ACPR for 8-user CDMA in presence of PD-HPA ................... 159

Table 6.5 ACPR for 128-OFDM in presence of PD-HPA $\ldots \ldots \ldots \ldots \ldots \ldots \ldots \ldots . .160$

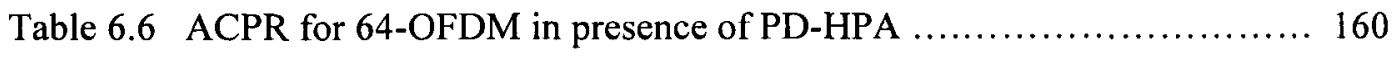

Table 6.7 ACPR for 16-OFDM in presence of PD-HPA $\ldots \ldots \ldots \ldots \ldots \ldots \ldots \ldots \ldots$ 


\section{LIST OF FIGURES}

Fig. 2.1 The AM-AM characteristic of Rapp's model for an SSPA ............ 21

Fig. 2.2 Block diagram of a typical PD-HPA, the AM-AM characteristics, and

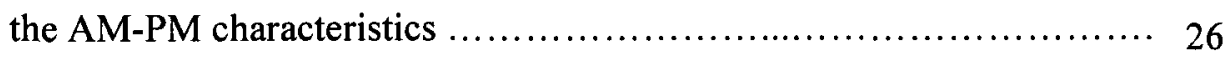

Fig. 2.3 Block diagram of a passband system ............................. 27

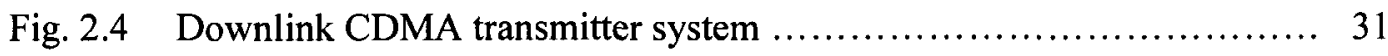

Fig. 2.5 Optimum receiver for synchronous transmission $\ldots \ldots \ldots \ldots \ldots \ldots \ldots \ldots \ldots \ldots \ldots$

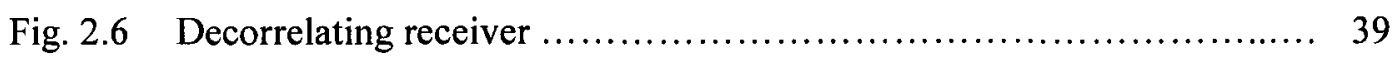

Fig. 2.7 Baseband block diagram of the OFDM transmitter ................... 43

Fig. 2.8 Baseband block diagram of the OFDM transmitter using IFFT .......... 43

Fig. 2.9 Baseband block diagram of the OFDM receiver using FFT ............ 45

Fig. 3.1 PD-HPA transfer function, (a) envelope of the input signal to PD-HPA $r(t)$, (b) envelope of the corresponding output signal $r_{d}(t)$ from PDHPA, and (c) envelope of the clipped signal portion $r_{c}(t) \ldots \ldots \ldots \ldots \ldots \ldots, 51$

Fig. 3.2 Analytic and simulated plots of $R_{E}, \sigma_{c}^{2}, \alpha_{0}$, and $\sigma_{d}^{2}$ versus IBO levels $\ldots 60$

Fig. 3.3 $\mathrm{SNR}_{\mathrm{NL}, k}$ versus $R_{E}$ and $\sigma_{c}^{2}$ at $\mathrm{SNR}_{\mathrm{AWGN}, k}=5 \mathrm{~dB} \ldots \ldots \ldots \ldots \ldots \ldots \ldots \ldots$ 
Fig. 3.4 Comparison of the analytic BER performance against measured BER from simulations for QPSK 64-user CDMA signal at different IBOs

Fig. 3.5 Comparison of the analytic BER performance against measured BER from simulations for 16QAM 64-user CDMA signal at different IBOs....

Fig. 3.6 Effect of $K / L$ on the BER performance of a 64-CDMA signal at IBO $\gamma=2 \mathrm{~dB}$

Fig. 3.7 Comparison of the analytic BER performance against measured BER from simulations for QPSK 256-OFDM signal at different IBOs

Fig. 3.8 BER versus SNR of QPSK 256-OFDM signal at different IBOs [43] .... 72

Fig. 3.9 SNR relative efficiency for a 256-OFDM signal at different IBOs ....... 75

Fig. 3.10 BER $_{\mathrm{NL}}$ versus $\mathrm{BER}_{\mathrm{AWGN}}$ for a $256-\mathrm{OFDM}$ signal at different IBOs ...... 76

Fig. 4.1 (a) Input signal $x(t)$ to PD-HPA, (b) corresponding output signal $x_{d}(t)$ from PD-HPA, and (c) clipped signal portion $x_{c}(t)$

Fig. 4.2 PSDs $S_{x_{d} x_{d}}(\omega), S_{x x}(\omega), S_{x x_{c}}(\omega), S_{x_{c} x}(\omega)$, and $S_{x_{c} x_{c}}(\omega)$ in (4.11) for:

(a) 16-user CDMA signal and (b) 64-channel OFDM signal

Fig. 4.3 Variances of the clipped signal portion

Fig. 4.4 Threshold crossing rates 98

Fig. 4.5 Average durations of fades 98 
Fig. 4.6 (a)-(d) PSDs for representations Rep. 1-4 of 8-user CDMA signal: the solid line represents input linear PSD $S_{x x}(\omega)$, the dotted line represents the output distorted PSD $S_{x_{d} x_{d}}(\omega)$, and the dashed line represents the PSD of the clipped signal portion $S_{x_{c} x_{c}}(\omega)$

Fig. 4.7 (a)-(d) PSDs for representations Rep. 5-8 of 16-user CDMA signal: the solid line represents input linear PSD $S_{x x}(\omega)$, the dotted line represents the output distorted PSD $S_{x_{d} x_{d}}(\omega)$, and the dashed line represents the PSD of the clipped signal portion $S_{x_{c} x_{c}}(\omega)$

Fig. 4.8 (a)-(d) PSDs for representations Rep. 9-12 of 64-user CDMA signal: the solid line represents input linear PSD $S_{x x}(\omega)$, the dotted line represents the output distorted PSD $S_{x_{d} x_{d}}(\omega)$, and the dashed line represents the PSD of the clipped signal portion $S_{x_{c} x_{c}}(\omega)$

Fig. 4.9 PSD plots for an 8-user CDMA signal with three different Walsh code assignments; (a) $W_{1}$, (b) $W_{2}$, and (c) $W_{3}$

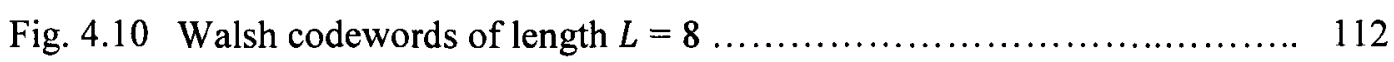

Fig. 4.11 PSD plots of different Walsh code sets with SRRC filtering .............. 114

Fig. 4.12 Walsh code assignment algorithm for $L=64 \ldots \ldots \ldots \ldots \ldots \ldots \ldots \ldots \ldots \ldots$

Fig. 4.13 PSD plots in presence of PD-HPA at different IBOs (dB): $W_{1}$ dotted curve, $W_{2}$ dashed curve and $W_{3}$ solid curve: (a) IBO $=1$, (b) $\mathrm{IBO}=2$, 
(c) $\mathrm{IBO}=3$, and (d) $\mathrm{IBO}=4$

Fig. 5.1 Instantaneous powers $r_{1}^{2}(t), r_{2}^{2}(t)$, and $r_{3}^{2}(t)$ of R1, R2, and R3, respectively

Fig. 5.2 Analytic and simulated plots of NLS versus IBO levels 130

Fig. 5.3 BER of (3.34) computed for R1-R7 versus IBO levels

Fig. 6.1 Block diagram of the SLM technique for a CDMA system

Fig. 6.2 Block diagram of the CSS technique for one subblock

Fig. 6.3 CCDFs of the normalized instantaneous power of an 8-user DS-CDMA signal

Fig. 6.4 Block diagram of the SLM technique for an OFDM system 145

Fig. 6.5 BER of 32-user CDMA signals in presence of PD-HPA 148

Fig. 6.6 BER of 16-user CDMA signals in presence of PD-HPA 148

Fig. 6.7 BER of 8-user CDMA signals in presence of PD-HPA 149

Fig. 6.8 BER of 128-OFDM signals in presence of PD-HPA 149

Fig. 6.9 BER of 64-OFDM signals in presence of PD-HPA 150

Fig. 6.10 BER of 16-OFDM signals in presence of PD-HPA 150

Fig. 6.11 PSDs of 32-user CDMA signals in presence of PD-HPA at (a) IBO = 1 
$\mathrm{dB}$, (b) IBO $=2 \mathrm{~dB}$, and (c) IBO $=3 \mathrm{~dB}$

Fig. 6.12 PSDs of 16-user CDMA signals in presence of PD-HPA at (a) IBO = 1 $\mathrm{dB}$, (b) $\mathrm{IBO}=2 \mathrm{~dB}$, and (c) $\mathrm{IBO}=3 \mathrm{~dB}$

Fig. 6.13 PSDs of 8-user CDMA signals in presence of PD-HPA at (a) IBO = 1 $\mathrm{dB}$, (b) $I B O=2 \mathrm{~dB}$, and (c) IBO $=3 \mathrm{~dB}$

Fig. 6.14 PSDs of 128-OFDM signals in presence of PD-HPA at (a) IBO $=1 \mathrm{~dB}$,

(b) IBO $=2 \mathrm{~dB}$, and (c) IBO $=3 \mathrm{~dB}$

Fig. 6.15 PSDs of 64-OFDM signals in presence of PD-HPA at (a) IBO $=1 \mathrm{~dB}$,

(b) IBO $=2 \mathrm{~dB}$, and (c) IBO $=3 \mathrm{~dB}$

Fig. 6.16 PSDs of 16-OFDM signals in presence of PD-HPA at (a) IBO $=1 \mathrm{~dB}$,

(b) IBO $=2 \mathrm{~dB}$, and (c) IBO $=3 \mathrm{~dB}$

Fig. 6.17 Typical HPA transfer functions for a TWTA and SSPA 163

Fig. 6.18 BER performance of 16-user CDMA signals in presence of TWTA 166

Fig. 6.19 BER Performance of 16-user CDMA signals in presence of SSPA 166 


\section{LIST OF ACRONYMS}

\begin{tabular}{|c|c|}
\hline $2 \mathrm{G}$ & Second generation \\
\hline 3G & third generation \\
\hline ACPR & adjacent channel power ratio \\
\hline ADSL & asymmetric digital subscriber line \\
\hline AM-AM & amplitude modulation to amplitude modulation \\
\hline AM-PM & amplitude modulation to phase modulation \\
\hline AWGN & additive white Gaussian noise \\
\hline BER & bit error rate \\
\hline BPSK & binary phase-shift keying \\
\hline CAD & computer aided design \\
\hline $\mathrm{CCDF}$ & complementary cumulative distribution function \\
\hline CDMA & code division multiple access \\
\hline CLT & Central Limit Theorem \\
\hline $\mathrm{CM}$ & cubic metric \\
\hline $\mathrm{CP}$ & cyclic prefix \\
\hline CRR & complexity reduction ratio \\
\hline CSS & cyclically shifted sequences \\
\hline $\mathrm{CW}$ & continuous wave \\
\hline
\end{tabular}




$\begin{array}{ll}\text { DFT } & \text { discrete Fourier transform } \\ \text { DPSK } & \text { differential PSK } \\ \text { DS-CDMA } & \text { direct-sequence CDMA } \\ \text { FDM } & \text { frequency division multiplexing } \\ \text { FFT } & \text { fast Fourier transform } \\ \text { FM } & \text { frequency modulation } \\ \text { FSK } & \text { frequency-shift keying } \\ \text { GSM } & \text { Global System for Mobile } \\ \text { HDSL } & \text { high bit rate digital subscriber line } \\ \text { HiperLAN } & \text { high performance radio LAN } \\ \text { HPA } & \text { high power amplifier } \\ \text { IBO } & \text { input back-off } \\ \text { ICI } & \text { interchannel interference } \\ \text { IDV } & \text { inverse DFT } \\ \text { IFF } & \text { intermediate frequency } \\ \text { instantaneous power variance }\end{array}$

IS-136 Interim Standard 136 


$\begin{array}{ll}\text { ISI } & \text { inter-symbol interference } \\ \text { ITU } & \text { international telecommunications union } \\ \text { LAN } & \text { local area network } \\ \text { LEO } & \text { low-earth orbiting } \\ \text { LPF } & \text { low pass filter } \\ \text { MEO } & \text { medium-earth orbiting } \\ \text { NLS } & \text { nonlinearity severity } \\ \text { MAI } & \text { multiple access interference } \\ \text { MPSK } & \text { M-ary PSK } \\ \text { MFSK } & \text { M-ary FSK } \\ \text { MQAM } & \text { M-level quadrature amplitude modulation } \\ \text { PSS } & \text { power spectral density } \\ \text { PSD } & \text { predinearity severity } \\ \text { OFDM } & \text { orthogonal frequency division multiplex } \\ \text { OFDMA } & \text { orthogonal frequency division multiple access } \\ \text { PAR } & \text { peak-to-average power ratio } \\ \text { PAPR } & \begin{array}{l}\text { peak-to-average power ratio } \\ \text { PCS }\end{array}\end{array}$


PTS partial transmitted sequences

QAM quadrature amplitude modulation

QPSK quadrature PSK

RF radio frequency

SLM selected mapping

SNR signal-to-noise ratio

SRRC square-root raised cosine filter

SS spread-spectrum

SSPA since solid state power amplifier

TWTA travelling wave tube amplifier

UMTS universal mobile telecommunications services

W-CDMA wideband CDMA

WiMAX worldwide interoperability for microwave access 


\section{LIST OF SYMBOLS}

\begin{tabular}{|c|c|}
\hline$A_{0}$ & limiting output amplitude of the amplifier \\
\hline$A_{k}$ & $k$ th symbol data in the in the OFDM block \\
\hline$a_{n}^{(k)}$ & $k$ th user symbol data in the $n$th symbol duration \\
\hline$B$ & bandwidth \\
\hline $\mathrm{BER}_{\mathrm{AWGN}}$ & BER in presence of $A W G N$ only \\
\hline $\mathrm{BER}_{\mathrm{NL}}$ & BER in presence of $A W G N$ and nonlinearity \\
\hline $\mathbb{C}^{(k)}$ & spreading code \\
\hline$c^{(k)}(t)$ & spreading waveform of the $k$ th user \\
\hline$c_{l}^{(k)}$ & lth chip in the spreading code \\
\hline$C(\because \cdot)$ & correlation metrics \\
\hline$C M$ & cubic metric \\
\hline$D(t)$ & domain of integration \\
\hline$E_{b}$ & signal energy per bit \\
\hline$E_{k}, E_{s}$ & signal energy per symbol \\
\hline$F[\cdot]$ & nonlinear distortion function of the amplifier chain \\
\hline$f_{k}$ & $k$ th subcarrier frequency \\
\hline$f_{x}(x)$ & probability density function \\
\hline
\end{tabular}




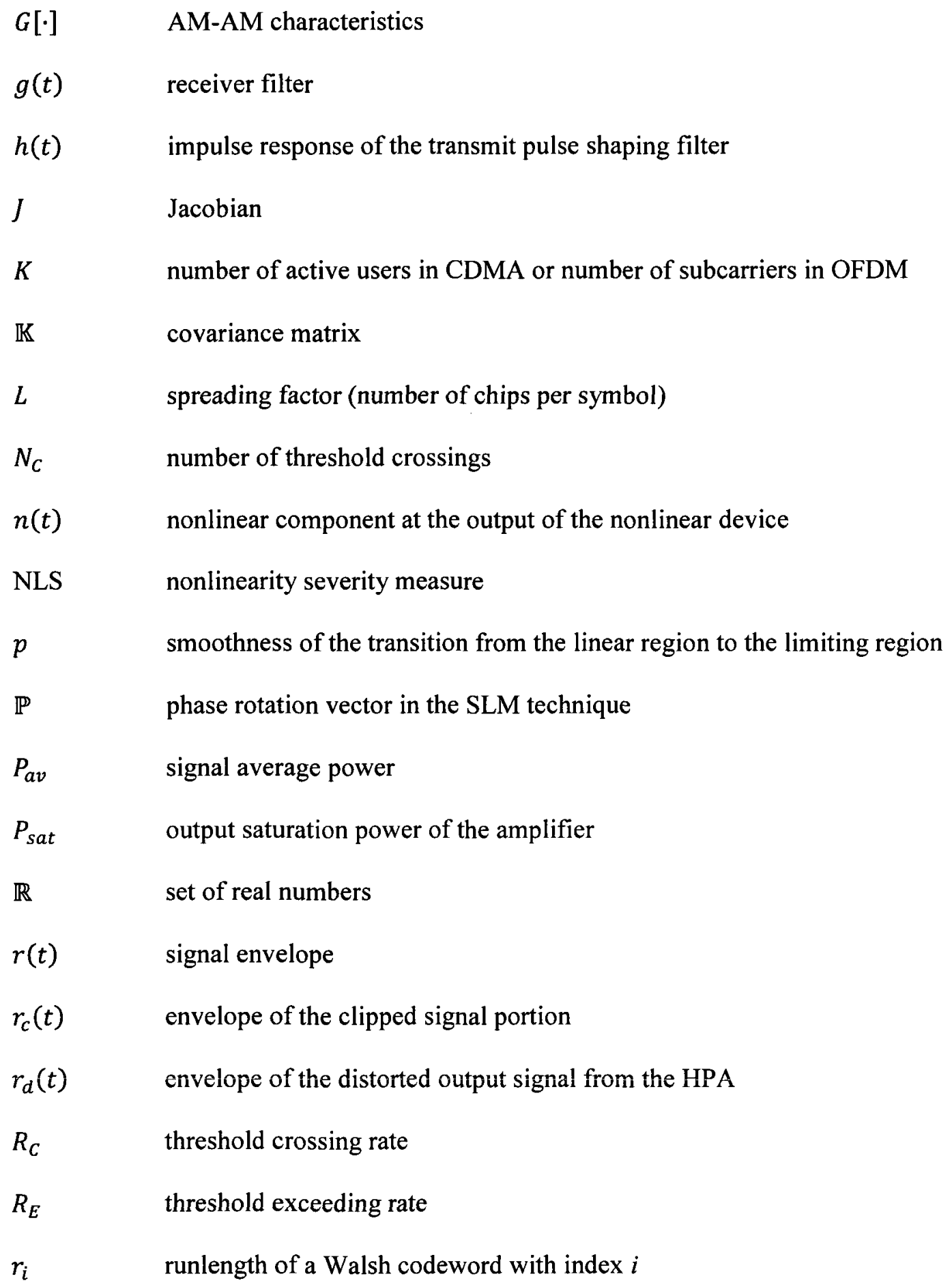


$R_{x x}(\tau) \quad$ autocorrelation function of the signal $x(t)$

$R_{x y}(\tau) \quad$ cross-correlation function of the signals $x(t)$ and $y(t)$

$\mathbb{R}_{s} \quad$ correlation matrix

$s_{k}(t) \quad$ signal component relative to the $k$ th user

SNR $_{\text {AWGN }}$ signal-to-noise ratio due to AWGN only

$\mathrm{SNR}_{\mathrm{NL}} \quad$ signal-to-noise ratio due to AWGN and nonlinearity

$S_{x x}(f) \quad$ PSD of the signal $x(t)$

$S_{x y}(f) \quad$ cross PSD function of the signals $x(t)$ and $y(t)$

T symbol duration

$T_{c} \quad$ chip duration

$T_{e} \quad$ effective symbol time

$T_{g} \quad$ guard time

$u(t) \quad$ received signal

$w(t) \quad$ AWGN

$w_{i}^{(k)} \quad$ Walsh function assigned to the $k$ th user with index $i$

$\bar{x}(t) \quad$ passband signals

$x_{c}(t) \quad$ clipped signal portion from the input signal $x(t)$ by the PD-HPA

$x_{d}(t) \quad$ distorted output signal from the HPA 


\begin{tabular}{|c|c|}
\hline$\alpha$ & small signal gain \\
\hline$\alpha_{0}$ & linear gain of the nonlinear device \\
\hline$\Delta f$ & subcarrier spacing \\
\hline$\gamma$ & IBO \\
\hline$\varepsilon_{k}$ & noise enhancement factor introduced by the decorrelating receiver \\
\hline$\zeta$ & saturation threshold of the amplifier \\
\hline$\eta(\gamma)$ & SNR relative efficiency \\
\hline$\theta(t)$ & angle (phase) of the input waveform to the amplifier \\
\hline$\theta_{d}(t)$ & angle of the distorted output signal \\
\hline$\lambda_{n}$ & $n$th spectral moment \\
\hline$\mu_{x}$ & mean of the $x(t)$ \\
\hline$\rho, \rho(t)$ & amplitude of the input waveform to the amplifier \\
\hline$\rho_{\max }$ & input voltage at the saturation point of the amplifier \\
\hline$\sigma_{|s|^{2}}^{2}$ & normalized instantaneous power variance \\
\hline$\sigma_{\dot{x}}^{2}$ & variance of the $\dot{x}(t)$ \\
\hline$\sigma_{x}^{2}$ & variance of the $x(t)$ \\
\hline $\bar{\tau}$ & mean threshold crossing duration \\
\hline$\tau_{i}$ & time duration of the crossing event \\
\hline$\tau^{(k)}$ & transmission delay for the $k$ th user \\
\hline$\varphi_{k}(t)$ & $k$ th subcarrier waveform in OFDM \\
\hline
\end{tabular}




$\begin{array}{ll}\Phi[\cdot] & \text { AM-PM characteristics } \\ \psi & \text { PAR } \\ \omega & \text { angular frequency } \\ \Lambda(\cdot) & \text { log-likelihood function }\end{array}$

$\begin{array}{ll}\delta(\cdot) & \text { delta function } \\ E\{\cdot\} & \text { statistical expectation } \\ \operatorname{erfc}(\cdot) & \text { complementary error function } \\ \operatorname{gcd}(\cdot, \cdot) & \text { greatest common divisor } \\ \operatorname{Im}\{\cdot\} & \text { imaginary } \\ Q(x) & \text { the area under the tail of the Gaussian pdf } \\ \operatorname{Re}\{\cdot\} & \text { real } \\ \operatorname{sgn}\{\cdot\} & \text { Sign function } \\ (\cdot)^{*} & \text { complex conjugate } \\ ((\cdot))_{N} & \text { the modulo- } N \text { operator } \\ {[\cdot]^{T}} & \text { transpose } \\ \otimes & \text { convolution } \\ \angle & \text { absolute } \\ |\cdot| & \end{array}$




\section{Chapter 1}

\section{INTRODUCTION}

high power amplifier (HPA) is an essential building block in wireless
communication systems. HPAs exhibit nonlinear characteristics when operated at high levels of input signal approaching the amplifier saturation.

Generally, for signals with constant envelope modulations, such as continuous wave $(\mathrm{CW})$, frequency modulation (FM), frequency-shift keying (FSK), and phase-shift keying (PSK), HPA nonlinearity is not a serious problem since the signal hits a single point in the characteristics of the HPA [1]. As the dynamic range of the input signal to the HPA increases traversing a larger region of the HPA characteristics, it is drastically affected by the inherent nonlinearity of the HPA resulting in two forms of distortion. First, in-band distortion that leads to degradation of the bit error rate (BER) performance. Second, additional out-of-band radiations known as spectral regrowth are created, which affect adjacent channels/radios.

In order to minimize the effects of such forms of distortion (BER degradation and spectral regrowth), the input signal to the HPA must be backed-off from the HPA saturation level. This input back-off (IBO) operation leads to a severe HPA inefficiency. For instance, an amplifier capable of 10 times the desired input power must be used when an IBO of $10 \mathrm{~dB}$ is required. With such a backing-off operation, the amplifier efficiency 
degrades significantly and its cost becomes a problematic issue, especially if we know that HPAs make up roughly $40 \%$ of a base station's cost [2]. This discrepancy between HPA linearity and efficiency poses a challenging design paradox for wireless systems designers.

This design paradox has generated much attention. Many efforts have been made to minimize the effects of nonlinear distortion and in turn improve the HPA efficiency. To the best of our study in the literature, two camps have achieved great contributions to minimize the effects of nonlinear distortion. The first camp has focused on the receiver side, while the second one has focused on the transmitter side. Also, collaborations between both camps have been always there.

At the receiver, nonlinear distortion can be compensated for by using channel equalization, inter-symbol interference (ISI) cancellation, or even better, maximumlikelihood sequence estimation. Equalization of channel nonlinearities first appeared in the pioneering work of Falconer [3] for voiceband data transmission and was later reconsidered by Benedetto et al. [4] for transmission of phase-modulated signals over nonlinear satellite channels. ISI cancellation attempts to identify the channel impulse response, synthesize a replica of the ISI affecting the current symbol, and subtract it from the demodulated signal sample prior to threshold detection [5]. 
Research dedicated to the transmitter side has pursued two approaches. The first approach focused on the HPA itself using linearization techniques such as feedback, feedforward, and predistortion. Adaptive linearization has been proposed, which can offer automatic compensation for amplifier nonlinearity and thus makes it possible to transmit multilevel modulated signals without incurring intolerable constellation distortions [6]. Also, analog/data predistortion has been proposed, which has become common practice in digital microwave radio systems $[5,7]$. The second approach focused on the input signal to the HPA by reducing the signal dynamic range. Decreasing the signal dynamic range allows the input signal to traverse a smaller region of the HPA characteristic, and hence less of the inherent nonlinearity of the HPA is revealed to the signal. Several techniques have been proposed to reduce the signal dynamic range such as clipping [8-10], companding [11-13], selected mapping (SLM) [14-16] and partial transmitted sequences (PTS) [17-19].

It is clear that techniques used at the receiver side attempt to fix the received signal after the nonlinear distortion has already occurred. These techniques are therefore considered as a cure for the symptoms rather than the causes of the problem itself, as Saleh and Salz observe, "since the source of nonlinearity is at the transmitter, it would appear logical to equalize the nonlinearity at the transmitter, where it occurs and where the transmitted bits are available" [6]. Moreover, improvements achieved using these techniques are usually limited by other factors such as the HPA nonlinearity severity itself, nonlinearities in the communication channel, and system complexity. Therefore, 
the work in this dissertation focuses on the transmitter side, specifically the input signal to the HPA, in order to minimize the nonlinear distortion before the signal even hits the amplifier.

\subsection{Research motivation and objective}

Over the decades, much research has been conducted to reduce the vulnerability of the amplifier input signal to nonlinearity. Such research often seeks to define a measure for the nonlinear distortion, which when reduced often allows the nonlinear distortion to also be reduced. Several measures have been proposed to quantify nonlinear distortion in relation to the input signal to the HPA such as the well known peak-toaverage power ratio (PAR) ${ }^{1}$, which is often used to characterize the signal dynamic range $[20,21]$. Also, the instantaneous power variance (IPV) $[22,23]$ and the cubic metric (CM) $[22,24]$ were proposed with the motivation of reducing the envelope fluctuations and the third-order modulation product, respectively.

While these measures have their use and have led to remarkable improvements, none of them has a close relation with the system performance. In other words, no clear mathematical relation exists between these measures and the resulting different forms of nonlinear distortion (BER degradation and spectral regrowth).

\footnotetext{
${ }^{1}$ PAR is the commonly used acronym for peak-to-average power ratio in CDMA, while in OFDM, PAPR is usually used. In this thesis, we use PAR as a unified acronym.
} 
Accordingly, the motivation behind this dissertation is to develop a new measure having a direct clear link with the system performance to characterize the nonlinear distortion. It is therefore imperative to explore which signal characteristics, at the HPA input, are responsible for performance degradation (BER degradation and spectral regrowth). Consequently, a measure formulated based on such characteristics would be more efficient for characterizing the nonlinear distortion. With this formulation, an efficient system in presence of a nonlinear amplifier chain can be achieved through the provision of:

- a potential estimate of the nonlinear distortion effects on the system performance, and

- the ability to minimize such effects by minimizing the signal characteristics contributing to performance degradation.

\subsection{Considerations and research methodologies}

The dissertation investigates two of the widely used signaling schemes nowadays; code division multiple access (CDMA) and orthogonal frequency division multiple access (OFDMA), which is based on orthogonal frequency division multiplexing (OFDM) technologies. These schemes appear to be particularly suited for the increased and continuous demands of more capacity, higher data rates, better quality of service, and robustness against multipath fading. CDMA and OFDM signals exhibit large dynamic range since they represent the sum of many contributions due to many users and many 
subcarriers, respectively, making them more susceptible to the amplifier nonlinearity. The considered CDMA system is assumed synchronous with equal users' powers.

A predistorter-HPA (PD-HPA) pair is used as a benchmark for the analyses of the nonlinear distortion effects. The PD-HPA has no nonlinear phase distortion, i.e. no phase insertion, and has an amplitude transfer function that is linear up to a certain level, after which it saturates (clips) the output signal. The amplifier chain is assumed memoryless. In a memoryless system, the output only depends on the input at the same instant of time. Memory effects affect low modulation frequencies causing scattering and/or hysteresis of the HPA transfer functions and asymmetry in the intermodulation distortion (IMD) products.

Both qualitative and quantitative research methodologies are utilized. In the quantitative research, the nonlinear distortion effects on the BER performance and spectral regrowth are mathematically analyzed.

In the BER performance analysis, an additive white Gaussian noise (AWGN) is considered as the channel impairment, where the signal-to-noise ratio (SNR) including the nonlinear distortion noise at the detector input is investigated, where the SNR represents the pivoting parameter in the BER performance of all types of receivers. Based on such analyses, new BER performance expressions (depending on the adopted 
modulation format and the receiver type) are developed that are functions in the signal characteristics at the input of the PD-HPA.

For spectral regrowth, the analysis of the power spectral density (PSD) at the PDHPA output shows that the PSD of the portion of the input signal that exceeds the PDHPA saturation threshold is primarily responsible for spectral regrowth. A piecewise analysis approach of such a signal portion is adopted to explore what other signal characteristics at the PD-HPA input contribute to spectral regrowth.

In the qualitative research, observations and logical interpretations from extensive simulations are presented to emphasize the effects of the established signal characteristics $^{2}$ on the system performance. Also, the developed analytical expressions of the established characteristics are validated through comparisons against data available in the literature and the measured values of such characteristics of the simulated signals. Finally, qualitative analysis of the transfer functions of typical nonlinear HPA models, with no predistorter involved in the amplifier chain, is conducted to show the applicability of the developed nonlinearity severity measure (NLS) measure to various HPA models.

Performance assessment of the developed NLS measure is conducted in comparison with PAR using two merits: the BER and the PSD. The SLM technique is

\footnotetext{
${ }^{2}$ The signal characteristics that contribute to nonlinear distortion effects (BER degradation and spectral regrowth).
} 
adopted as a platform for performance assessment, where several representations of the signal to be transmitted are generated and the one with the minimal PAR (or NLS) is selected for transmission. Additionally, the performance of the NLS measure is examined with different HPA models (no predistorter is involved in the amplifier chain). Two HPA models for travelling wave tube amplifier (TWTA) and solid state power amplifier (SSPA) are investigated.

\subsection{Summary of contributions}

In this dissertation, we present an analysis technique for the solution of the nonlinear distortion effects on CDMA and OFDM signals passed through a nonlinear amplifier chain. In such a technique, we explore the characteristics of the HPA input signal, associated with the HPA characteristics, which contribute to nonlinear distortion. The solution provides potential insight into how the HPA and the signal interact to produce BER degradation and spectral regrowth. Accordingly, the following contributions are achieved.

- Based on the analysis of the system SNR and the BER performance at the input of the receiver's detector, it is established that the input signal characteristics contributing to BER degradation due to HPA nonlinearity are: the rate at which the input signal exceeds the PD-HPA saturation threshold and the variance of the portion of the input signal that exceeds that threshold. 
- Based on the analysis of the PSD at the output of the amplifier, it is established that the input signal characteristics contributing to spectral regrowth due to HPA nonlinearity are: the variance of the signal portion exceeding the PD-HPA saturation threshold, the rate at which the input signal crosses that threshold, and the time durations of the crossing events.

- Based on the established signal characteristics, a new nonlinearity measure, NLS, is developed. The developed NLS parameter represents an efficient quality measure for the potential nonlinear distortion, as an alternative to the existing measures (PAR, IPV, and CM). Also, being a function in the IBO level gives the NLS measure a superior advantage as it can be adjusted to the IBO level required to work at according to design demands. Additionally, this measure can be used as a selection criterion in search and optimization techniques, like SLM and PTS, to select the best signal representation to be transmitted.

During the research work in this dissertation, other relevant contributions have been achieved that also have significant practical applications. In the following, we summarize such contributions.

- As a special case for synchronous CDMA signals with orthogonal spreading codes, it is shown that the PSD of the CDMA signal depends primarily on the PSDs of the spreading functions assigned to each user in the system as it is 
approximately a scaled sum of the PSDs of the spreading functions assigned to all users.

- For the particular case of using Walsh codes in synchronous CDMA signals, it is shown that the PSD of Walsh functions and the PSD of the sum of Walsh functions depend on the specific properties of Walsh codes. Based on such properties of Walsh codes, an effective Walsh code assignment algorithm is presented to minimize spectral regrowth.

- Finally, a low complexity technique is presented as an alternative to SLM and PTS techniques to reduce computational burden and exhaustive search. The concept of the presented technique is based on cyclic shifts of the symbol data sequences.

\subsection{Thesis organization}

This thesis consists of seven chapters as follows. Chapter 2 introduces the basic principles and the theoretical background information necessary for the rest of the work in this thesis. A simple way to understand the nonlinear effect on the input signal to the HPA is introduced. The HPA characteristics and the parameters used for HPAs modeling are explained, and examples for four of the currently known HPA models, are presented. 
Finally, the basics of the system models under investigations, CDMA and OFDM, are discussed.

Chapter 3 presents the analyses of the nonlinear distortion effects on BER performance for CDMA and OFDM. The signal characteristics contributing to BER degradation are established. Also, analytic expressions for such characteristics and the BER performance are developed. Validation of the developed analytical expressions thru comparisons versus measurements from simulations and available data in the literature is also presented.

Chapter 4 separates the PSD at the HPA output into individual components representing both the in-band distortion and the out-of-band distortion that represents spectral regrowth. Through a piecewise analysis of the signal portion that is clipped from the input signal by the PD-HPA saturation threshold, the signal characteristics contributing to spectral regrowth are determined. Also, CDMA signals with orthogonal spreading codes are studied as a special case, highlighting the effect of the specific properties of Walsh codes on spectral regrowth.

Chapter 5 presents a brief review on the existing measures used to characterize the nonlinear distortion showing that such measures are not mathematically related to the system performance. Based on the signal characteristics developed in Chapter 3 and 
Chapter 4 that represent the main contributors to BER degradation and spectral regrowth, the NLS measure is established.

Chapter 6 assesses the performance of the developed NLS measure through comparison against PAR as two selection criteria in SLM technique. Also, performance assessments of the NLS measure are conducted to show the efficiency of the NLS measure with different HPA models.

Chapter 7 concludes the dissertation with a summary of the findings of this thesis in terms of advantages and disadvantages. Future areas of research are discussed as a follow-up to the work presented in this thesis. 


\section{Chapter 2}

\section{THEORETICAL BACKGROUND}

This chapter explains the basic nonlinearity problem of HPAs. It also provides a
brief survey on HPA characteristics and modeling. Then the effect of nonlinearity in both the passband and baseband nonlinear models is introduced. Finally, the system models under investigation (CDMA and OFDM) in this dissertation are presented.

\subsection{The nonlinearity problem}

An HPA is an essential part of a transmitter, which in turn is necessary for all wireless communication systems, including wireless LAN devices, cellular phones, wireless base stations, radio and TV stations, satellites and so forth. HPAs exhibit nonlinear characteristics when operated at high levels of input signal approaching the amplifier saturation. Such a lack in the linearity of the HPA characteristics creates serious distortion in the transmitted signal. This distortion leads to poor performance in terms of BER degradation and spectral regrowth.

In order to understand the nonlinear effect of an HPA, consider the following example of an input signal to the HPA composed of two cosine waves of equal amplitude: 


$$
v_{i}(t)=v \cos \omega_{1} t+v \cos \omega_{2} t
$$

These two tones are designed in a way that the frequency spacing between them is much smaller than the radio frequency (RF) of either tone. Assume the nonlinear model of the HPA is defined by the following $n$ th-order polynomial:

$$
v_{0}(t)=a_{1} v_{i}(t)+a_{2} v_{i}^{2}(t)+a_{3} v_{i}^{3}(t)+\cdots+a_{n} v_{i}^{n}(t)
$$

By substituting the two tone input defined by (2.1) into the characteristic equation of the HPA defined by (2.2), the output signal will therefore be:

$$
\begin{aligned}
v_{0}(t)= & a_{1} v\left(\cos \omega_{1} t+\cos \omega_{2} t\right) \\
& +a_{2} v^{2}\left(\cos \omega_{1} t+\cos \omega_{2} t\right)^{2} \\
& +a_{3} v^{3}\left(\cos \omega_{1} t+\cos \omega_{2} t\right)^{3} \\
& +\cdots \\
& +a_{n} v^{n}\left(\cos \omega_{1} t+\cos \omega_{2} t\right)^{n}
\end{aligned}
$$

Some important facts should be emphasized from the expansion of (2.3)

- Each $n$ th-order term of the polynomial will produce a number of tones equal to $2 n$, and each tone will be scaled by a factor of $x_{i} a_{n} v^{n}$, where $x_{i}$ is a specific fraction that scales the tone and has been obtained from the expansion. 
- The tones from each $n$ th-order term of the polynomial are centered at frequencies that are products of that order. For example, the products of the third-order term are 6 tones centered at $3 \omega_{1}, 3 \omega_{2}, 2 \omega_{1} \pm \omega_{2}$, and $2 \omega_{2} \pm \omega_{1}$, while a fourth-order term produces 8 tones centered at $4 \omega_{1}, 4 \omega_{2}, 3 \omega_{1} \pm \omega_{2}, 3 \omega_{2} \pm \omega_{1}$ and $2 \omega_{2} \pm$ $2 \omega_{1}$.

- All tones produced from even-order terms are at frequencies distant from the main band at which the original two tones are located, which can be ignored by bandpass filtering.

Expanding (2.3), and dropping the dependency on time for simplicity, yields

$$
\begin{aligned}
v_{0}(t)=a_{2} v^{2} & +\left[a_{1} v+\frac{9}{4} a_{3} v^{3}\right] \cos \omega_{1}+\left[a_{1} v+\frac{9}{4} a_{3} v^{3}\right] \cos \omega_{2}+a_{2} v^{2} \cos \left(\omega_{1}+\omega_{2}\right) \\
& +a_{2} v^{2} \cos \left(\omega_{2}-\omega_{1}\right)+\frac{3}{4} a_{3} v^{3} \cos \left(2 \omega_{1}-\omega_{2}\right)+\frac{3}{4} a_{3} v^{3} \cos \left(2 \omega_{2}-\omega_{1}\right) \\
& +\frac{3}{4} a_{3} v^{3} \cos \left(2 \omega_{1}+\omega_{2}\right)+\frac{3}{4} a_{3} v^{3} \cos \left(2 \omega_{2}+\omega_{1}\right)+a_{2} v^{2} \cos 2 \omega_{1} \\
& +a_{2} v^{2} \cos 2 \omega_{2}+\frac{3}{4} a_{3} v^{3} \cos 3 \omega_{1}+\frac{3}{4} a_{3} v^{3} \cos 3 \omega_{2}+\cdots
\end{aligned}
$$

Clearly from the expansion in (2.4), when the input signal has a variable envelope, the output products that lie on the same frequencies of the input signal $\left(\omega_{1}\right.$ and $\omega_{2}$ ) are distorted by the generated exponents and the scaling factors produced from oddorder terms. This signal distortion leads to BER degradation at the receiver. In addition to 
the signal distortion due to these output products, there are other important products that greatly affect the original signal. These products are termed the intermodulation (IM) products. These IM products mainly emerge from the odd-order terms of the polynomial and are near the input signal band. In this fashion, each odd-order term will add to the output spectrum of the HPA. This increase in the out-of-band spectrum is called spectral regrowth. Theoretically, odd-order intermodulation products never cease to contribute to the output spectrum. In practice, however, contributions from high-order IM products are usually neglected. It is common practice to take into consideration only IM3 products, but sometimes IM5 and even IM7 products have been used for more precise and complex calculations.

In order to employ the HPA in a more efficient manner, various methods can be exploited to linearize the input/output power relationship of the HPA. These methods generally provide a better HPA efficiency along with lower levels of signal distortion. In most cases, however, in order to linearize an HPA one must first characterize and model the device. There are various ways to perform these tasks. The most common are reviewed in the following sections.

\subsection{HPA characteristics}

Consider $x(t)$ is the baseband input signal to the HPA and $y(t)$ is the baseband output signal. Experimental evidence [1 and the references therein] shows that both the 
output amplitude $|y(t)|$ and the output phase deviation $\angle y(t)-\angle x(t)$ depend on the input amplitude $|x(t)|$ alone. The relationships between the output and the input of the HPA are given by

$$
\begin{aligned}
y(t) & =F[x(t)], \\
|y(t)| & =G[|x(t)|],
\end{aligned}
$$

and

$$
\angle y(t)=\Phi[|x(t)|]+\angle x(t)
$$

where $F[\cdot]$ is the nonlinear distortion function of the amplifier, $G[\cdot]$ is the amplitude modulation to amplitude modulation (AM-AM) characteristic, and $\Phi[\cdot]$ is the amplitude modulation to phase modulation (AM-PM) characteristic.

It is worth shedding light on memory effects that arise in HPAs when wideband signals are present at the HPA input $[7,25,26]$. In a memoryless system, the output only depends on the input at the same instant of time. There are two main types of memory effects: (i) electrical memory effects and (ii) thermal memory effects. Electrical memory effects are caused by varying envelope and fundamental or second harmonic impedances at different modulation frequencies. With careful design, electrical memory effects can be limited to those caused by bias networks [27]. Thermal memory effects are caused by electro-thermal coupling, and these affect low modulation frequencies up to a few 
megahertz (MHz) [27]. Frequency dependent behavior of the HPA can often be ignored for narrowband signals, but they must be accounted for when wideband signals are amplified. Symptoms of the nonlinear memory effect include [1] (i) scattering and/or hysteresis of the AM-AM and AM-PM curves and (ii) asymmetry in the intermodulation distortion (IMD) products.

A strictly memoryless HPA has AM-AM characteristics but no AM-PM characteristics (i.e., $\Phi[|x(t)|]$ is a constant). A quasi-memoryless HPA has both AM-AM and AM-PM characteristics [28]. An ideal linear HPA would have $G[|x(t)|]=A|x(t)|$ and $\Phi[|x(t)|]=\theta$ for all $|x(t)|$ between 0 and the saturation point of the HPA, where $A$ and $\theta$ are constants.

\subsection{HPA models}

HPA characteristics and nonlinearities need to be described and represented with great accuracy. This is because through these representations, one can fully understand the behavior of any HPA and can therefore use the information to find and rectify any errors that are caused by these nonlinearities. It is common to term these representations that describe the behavior of HPAs as "models." There are two ways of modeling an HPA: either by representing its AM-AM and AM-PM characteristics, or by illustrating the behavior of its inphase and quadrature components. Since modeling the AM-AM and AM-PM characteristics of an HPA is more common and indeed a logical step following 
the characterization methods described in the previous section, these types of models will be the focus of this section.

In order to model the AM-AM and AM-PM characteristics of an HPA, it is necessary to represent the data obtained from the characterization process in a continuous form. Such forms are typically mathematical models, which can be represented as graphs for illustration purposes. There are various models that have been used in the past, as well as some that are more recent. Each model varies in complexity and accuracy and each has been used for different purposes. In the following, we briefly describe some of the well known HPA models.

\subsubsection{Saleh's model}

In 1981, Adel A. M. Saleh created a method for modeling TWTAs [7]. This method was created to describe discrete readings obtained from one of the characterization processes. Saleh's model has become very popular because of its simplicity and because it can be used to model either the AM-AM and AM-PM characteristics or the in-phase and quadrature components. The model is still used today for many modeling purposes as well as a reference of comparison for newly developed models. 
Saleh developed two simple formulas to model the AM-AM and AM-PM characteristics of a TWTA:

$$
\begin{gathered}
G(\rho)=\frac{\alpha_{a} \rho}{1+\beta_{a} \rho^{2}} \\
\Phi(\rho)=\frac{\alpha_{\phi} \rho^{2}}{1+\beta_{\phi} \rho^{2}}
\end{gathered}
$$

where $G(\rho)$ and $\Phi(\rho)$ are, respectively, the AM-AM and AM-PM characteristics of the amplifier being modeled, with reference to $\rho$, the amplitude of the input waveform. The constants $\alpha_{a}, \beta_{a}, \alpha_{\phi}$ and $\beta_{\phi}$ are uniquely defined for each amplifier and are obtained by employing standard minimum mean-square-error curve fitting procedures on the data obtained in the characterization process.

Saleh's model has proven to be accurate when modeling many TWTAs. It also provides a simple and quick method of modeling amplifiers by using the data collected when characterizing them. However, since SSPAs have different characteristics than TWTAs, this method might not always be a reliable one.

\subsubsection{Rapp's model}

The typical SSPA has a more linear behavior in the small signal region than a TWTA. For large inputs, the amplitude transfer function tends to a maximum limiting 


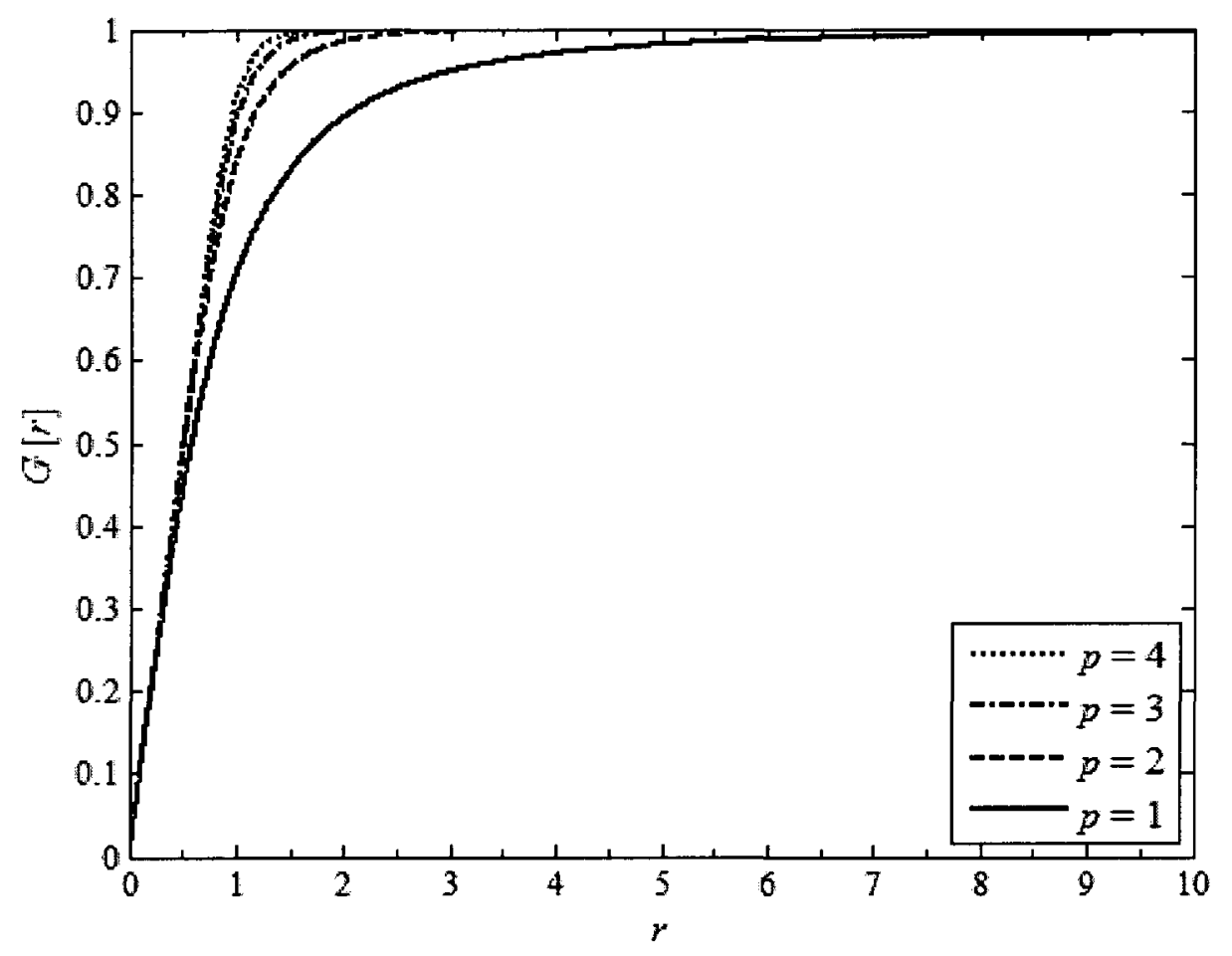

Fig. 2.1 The AM-AM characteristic of Rapp's model for SSPA.

value, produced by the current and voltage clipping. The AM-PM characteristic of the SSPA is assumed to be small enough, so that it can be neglected $(\Phi(\rho) \cong 0)[29]$. In [29], Rapp developed a simple formula to model the AM-AM characteristics of an SSPA that is near identical to Cann's model [30]:

$$
G(\rho)=\frac{\alpha \rho}{1+\left(\left(\frac{\alpha \rho}{A_{0}}\right)^{2 p}\right)^{\frac{1}{2 p}}}, \quad p>0
$$


where $A_{0} \geq 0$ is the limiting output amplitude, $\alpha \geq 0$ is the small signal gain, and the smoothness of the transition from the linear region to the limiting region is controlled by the parameter $p$. The AM-AM characteristic of such a model for SSPA is shown in Fig. 2.1, with $A_{0}=\alpha=1$.

\subsubsection{Polynomial model}

Polynomials have been used extensively to model HPAs in many applications. The polynomial model, sometimes referred to as a power series representation, creates a simple and highly effective technique for modeling HPAs. It is especially useful for HPA linearization purposes by means of predistortion.

An HPA's AM-AM and AM-PM polynomial models can be expressed as power series representations of the following form:

$$
\begin{aligned}
& G(\rho)=a_{0}+a_{1} \rho+a_{2} \rho^{2}+a_{3} \rho^{3}+a_{4} \rho^{4}+a_{5} \rho^{5}+\cdots \\
& \Phi(\rho)=b_{0}+b_{1} \rho+b_{2} \rho^{2}+b_{3} \rho^{3}+b_{4} \rho^{4}+b_{5} \rho^{5}+\cdots
\end{aligned}
$$

where $a_{i}$ and $b_{i}, i=0,1,2, \ldots$, are the coefficients of power series that relates the amplitude of the input waveform $\rho$ to the AM-AM characteristic $G(\rho)$ and the AM-PM characteristic $\Phi(\rho)$, respectively. 
Equations (2.11) and (2.12) take on a general form that can be used to model any type of nonlinear behavior including that of HPAs [31, 32]. With the help of polynomial curve fitting tools, typically computer aided design (CAD) software, the appropriate values of the $a$ and $b$ coefficients can be found so that equations (2.11) and (2.12) best fit the data obtained from the characterization process. It should be noticed, however, that since (2.11) and (2.12) are infinite series, they must be truncated at some point in order for the CAD software to compute the algorithm. Customarily, this truncation is chosen with the aim of creating a polynomial of odd-order, typically of 3rd, 5 th or 7 th order. In general, a polynomial of larger order will produce a more accurate model of the HPA but will require more resources and processing power to compute. It is customary that both the AM-AM and AM-PM polynomial models are of the same order.

The polynomial model creates an easy and quick method of modeling HPAs. It also provides the ability to achieve higher accuracy by choosing a higher-degree polynomial, or less complexity by choosing a lower-degree polynomial, according to design demands.

\subsubsection{PD-HPA model}

Predistortion can be defined, as the name implies, as the process of distorting the input signal prior to the HPA so that when the signal passes through the HPA, much of the nonlinearity introduced by the HPA is removed. This cascaded operation of the 
predistorter (PD) and the HPA is termed PD-HPA. Predistortion could be adaptive to automatically cope with any time-variations of the HPA response due to temperature variations and aging. There are two basic types of predistortion techniques: data predistortion and analog signal predistortion at the intermediate frequency (IF) (or RF) stage of the transmitter [5]. Both types require accurate knowledge of the AM-AM and AM-PM characteristics of the HPA in order to precisely model the HPA. From such a model, one would be able to derive an inverse model for the predistorter, which when used in cascade with the HPA would create a more linear system.

Based on the AM-AM and AM-PM characteristics of the HPA, data predistortion consists of appropriately modifying (distorting) the input signal constellation so as to obtain the desired constellation after the HPA nonlinearity [5]. Such a predistortion scheme was described by Saleh and Salz [6]. On the other hand, analog predistortion consists of placing a nonlinear circuit at the IF or the RF stage of the transmitter that attempts to perfectly match the inverse characteristics of the HPA response [5].

In order to view the operation of the PD-HPA, consider the input signal to the HPA that has the form

$$
x(t)=\rho(t) \cos \left(\omega_{c} t+\theta(t)\right)
$$

where $\rho(t)$ and $\theta(t)$ represent the instantaneous amplitude and phase of $x(t)$ at a carrier frequency $\omega_{c}$, respectively. Then the output of the HPA can be written as 


$$
z(t)=G[\rho(t)] \cos \left(\omega_{c} t+\theta(t)+\Phi[\rho(t)]\right)
$$

where $G[\cdot]$ and $\Phi[\cdot]$ represent the AM-AM and AM-PM characteristics (also called phase insertion) of the HPA, respectively. In the same way, if $x(t)$ was the input to the predistorter, the output of this would take the form

$$
y(t)=A[\rho(t)] \cos \left(\omega_{c} t+\theta(t)+\Psi[\rho(t)]\right)
$$

where the amplitude and phase characteristic functions of the predistorter $A[\cdot]$ and $\Psi[\cdot]$ are created to eliminate all HPA nonlinearity and create a PD-HPA cascade of the form:

$$
\begin{aligned}
G[A(\rho)]= & \left\{\begin{array}{ll}
\alpha \rho, & 0 \leq \rho<\rho_{\max } \\
\alpha \rho_{\max }, & \rho>\rho_{\max }
\end{array},\right. \\
& \Phi[A(\rho)]=-\Psi[\rho],
\end{aligned}
$$

where $\rho_{\max }$ is the value of the input voltage at the saturation point of the HPA, $\alpha$ is the voltage gain of the PD-HPA cascade and the dependency on time has been dropped for simplicity.

The above-mentioned PD-HPA creates a perfectly linear power gain and a zero phase insertion at the output of the HPA that represents the ideal predistortion. The block diagram of a typical PD-HPA, the AM-AM characteristics, and the AM-PM 
characteristics are depicted in Fig. 2.2. In practice, however, due to physical limitations at the time of implementation, only an approximation of the ideal PD can be created.
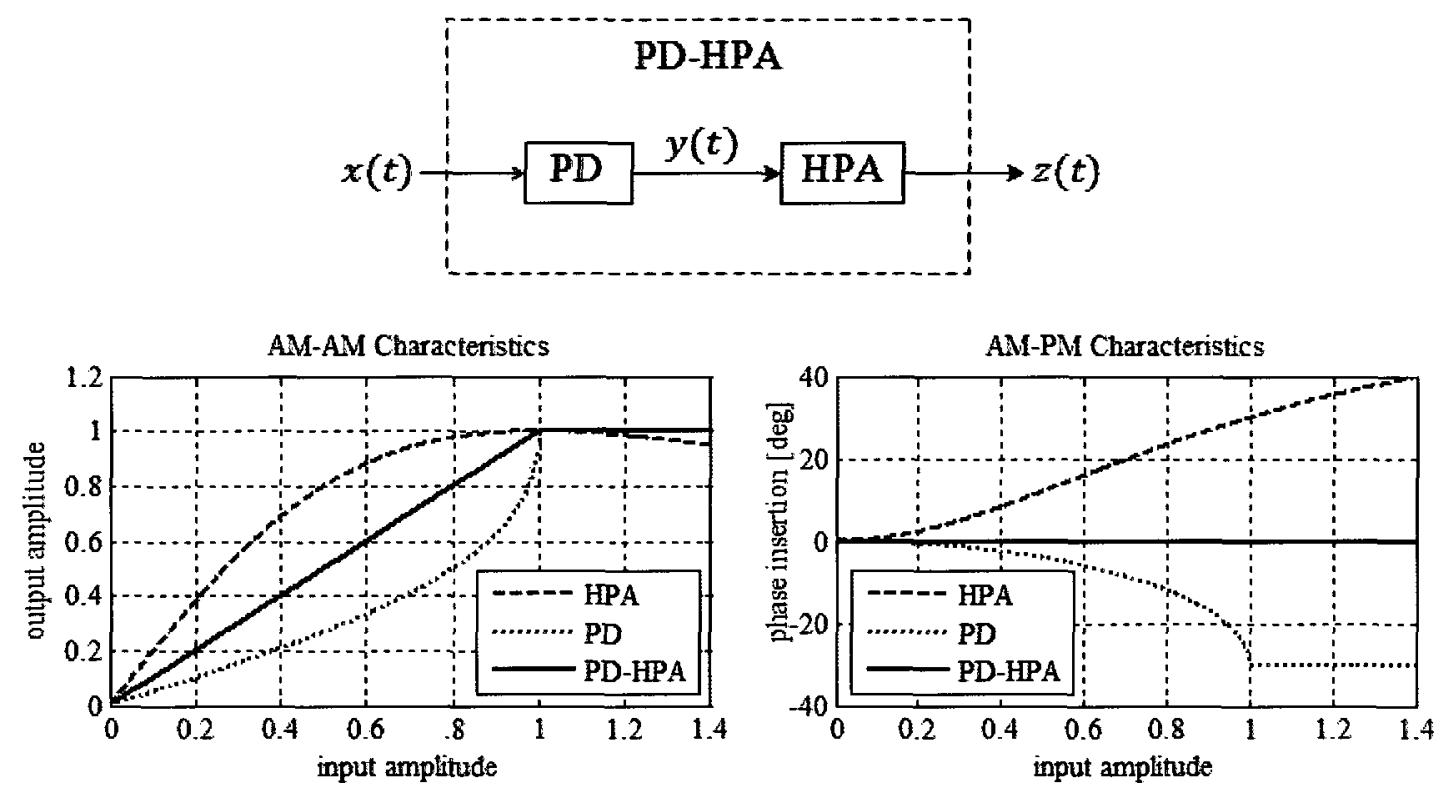

Fig. 2.2 Block diagram of a typical PD-HPA, the AM-AM characteristics, and the AMPM characteristics.

\subsection{Baseband/passband nonlinearity}

It is convenient for the analysis of the nonlinear effects to introduce both the passband and baseband nonlinear models. The block diagram in Fig. 2.3 depicts the upconversion of the complex-valued baseband signal $x(t)$ to the passband, the 
amplification of the real-valued passband input $\bar{x}(t)$, and the down-conversion of the passband output $\bar{y}(t)$ into a complex-valued baseband signal $y(t)[1]$.

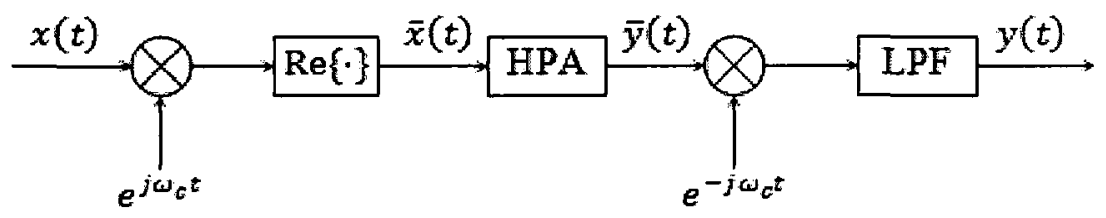

Fig. 2.3 Block diagram of a passband system.

The real-valued passband input (modulated carrier) signal $\bar{x}(t)=\operatorname{Re}\left\{x(t) e^{j \omega_{c} t}\right\}$, where $\omega_{c}$ is the carrier frequency, is applied to a nonlinear circuit with a nonlinear gain characteristic, $F[\bar{x}(t)]$. The nonlinear gain characteristic is assumed to be a bandpass nonlinearity containing no significant memory within the bandwidth of the modulation. Thus, the AM-AM and AM-PM nonlinearities respond instantaneously to amplitude changes from the modulated carrier signal. It is important to note that the AM-AM and AM-PM responses represent the transfer characteristic of the input to the desired output frequency. A complex power series expansion is used to model the instantaneous AM$\mathrm{AM}$ and $\mathrm{AM}-\mathrm{PM}$ characteristics:

$$
\begin{aligned}
\bar{y}(t) & =F[\bar{x}(t)]=\bar{a}_{1} \bar{x}(t)+\bar{a}_{3} \bar{x}^{3}(t)+\bar{a}_{5} \bar{x}^{5}(t)+\cdots+\bar{a}_{N} \bar{x}^{N}(t) \\
& =\sum_{l=0}^{(L-1) / 2} \bar{a}_{2 l+1} \bar{x}^{2 l+1}(t)
\end{aligned}
$$


It can be shown [33] that the baseband equivalent model is

$$
\begin{aligned}
y(t) & =F[x(t)]=x(t) \sum_{n=0}^{N} a_{2 n+1}|x(t)|^{2 n} \\
& =\sum_{n=0}^{N} a_{2 n+1} x^{n+1}(t) x^{* n+1}(t)
\end{aligned}
$$

where

$$
a_{2 n+1}=\frac{1}{2^{2 n}}\left(\begin{array}{c}
2 n+1 \\
n
\end{array}\right) \bar{a}_{2 n+1}
$$

In general, the power series contains all powers of the input signal; however, as mentioned previously, the even order terms generate output components at frequencies distant apart from the signal bandwidth that can be eliminated by filtering. Further analysis for nonlinear amplifiers with memory can be found in [1] and [33].

\subsection{Signaling systems under investigation}

CDMA and OFDM represent two of the most widely used signaling schemes nowadays. They appear to be most suited for the growing demands of larger capacity, higher data rates and quality of service, and robustness to multipath fading. Unfortunately, CDMA and OFDM signals exhibit large dynamic range making them 
more susceptible to amplifier nonlinearity. In this dissertation, we investigate the effect of the amplifier nonlinearity on these signals. In the following subsections, we introduce a review of the basics of CDMA and OFDM systems.

\subsubsection{CDMA basics}

CDMA is a form of spread-spectrum (SS), a family of digital communication techniques that have been used in military applications for many years. The core principle of spread spectrum is the use of noise-like carrier waves, and, as the name implies, bandwidths much wider than that required for simple point-to-point communication at the same data rate. Originally there were two motivations: immunity to interference and jamming, or hiding the fact that communication was even taking place, sometimes called low probability of intercept. It has a history that goes back to the early days of World War II. The use of CDMA for civilian mobile radio applications was proposed theoretically in the late 1940 's, but the practical application in the civilian marketplace did not take place until 40 years later. Commercial applications became possible because of two evolutionary developments. One was the availability of very low cost, high density digital integrated circuits, which reduce the size, weight, and cost of the subscriber stations to an acceptably low level. The other was the realization that optimal multiple access communication requires that all user stations regulate their transmitter powers to the lowest that will achieve adequate signal quality. In 1999, the international telecommunications union (ITU) selected direct-sequence CDMA (DS-CDMA) as the 
industry standard for new $3 G$ wireless systems. The eventual $3 G$ evolution for $2 \mathrm{G}$ cdmaOne systems led to cdma2000, while the eventual $3 \mathrm{G}$ evolution for $2 \mathrm{G}$ Global System for Mobile (GSM) and Interim Standard 136 (IS-136) systems led to wideband CDMA (W-CDMA), also called universal mobile telecommunications services (UMTS). Nevertheless in satellite systems, several personal communication systems (PCSs) based on low-earth orbiting (LEO) and medium-earth orbiting (MEO) satellite constellations have selected DS-CDMA as the access scheme. DS-CDMA is selected for these systems because of its noteworthy low-power flux density emission, time-domain signal discrimination, and interference resilience [34].

CDMA systems are multiuser communication systems in which a large number of users share a common communication channel to transmit information to a receiver. The common channel may be an uplink (reverse) channel from user station to the base station or a downlink (forward) channel from base station to the user(s) station. In CDMA, each user is assigned a distinct spreading waveform, which allows the corresponding user's receiver to demodulate the transmitted message among multiple users transmitting simultaneously. Generally, in the uplink, the presence of nonlinear devices at the transmitter represents a minor issue. On the contrary, in the downlink, the sum of many components coming from different synchronous or asynchronous users forms a signal with a large dynamic range, which could be severely distorted by the nonlinear characteristics of the HPA. In this dissertation, we are interested in investigating the effects of nonlinear distortion due to HPA; hence, we shall focus on the downlink system. 


\subsubsection{Transmitter system}

In a $\mathrm{CDMA}^{1}$ system with $K$ active users, the signal component relative to the $k$ th user can be expressed by

$$
s_{k}(t)=\sqrt{E_{k}} \sum_{n=-\infty}^{\infty} a_{n}^{(k)} c^{(k)}\left(t-n T-\tau^{(k)}\right)
$$

where $E_{k}$ is the $k$ th user's signal energy per symbol, $T$ is the symbol duration, and $\left\{\tau^{(k)}\right\}$ are the transmission delays, which satisfy the condition $0 \leq \tau^{(k)}<T$ for all $k$. In case of synchronous transmission, $\tau^{(k)}=0$ for all $k . a_{n}^{(k)}$ are the $k$ th user's symbol data in the $n$th symbol duration. The block diagram of the downlink CDMA transmitter system is depicted in Fig. 2.4 .

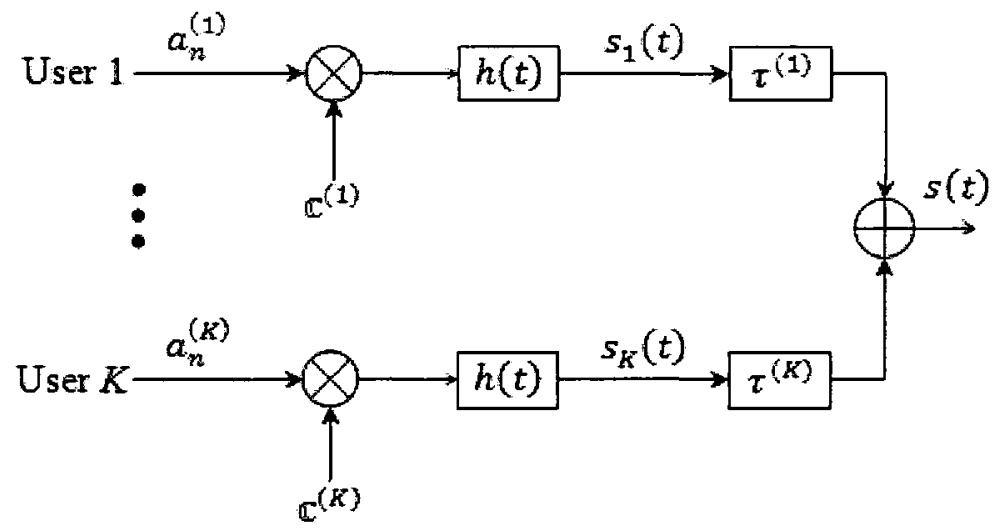

Fig. 2.4 Downlink CDMA transmitter system.

\footnotetext{
${ }^{1}$ We henceforth use the acronyms CDMA and DS-CDMA interchangeably
} 
In (2.21),

$$
c^{(k)}(t)=\sum_{l=0}^{L-1} c_{l}^{(k)} h\left(t-l T_{c}\right)
$$

is the spreading waveform obtained with the spreading code $\mathbb{C}^{(k)}=\left[c_{0}^{(k)}, \ldots, c_{L-1}^{(k)}\right]^{T}$ and with cross correlations $\rho_{i j}(\tau)=\int_{0}^{T} c^{(i)}(t) c^{(j)}(t-\tau) d t, L$ is the spreading factor (number of chips per symbol), $T_{c}=T / L$ is the chip duration, and $h(t)$ is the impulse response of the transmit pulse shaping filter. Without loss of generality, the spreading waveforms are assumed to have unit energy, i.e.,

$$
\int_{0}^{T}\left[c^{(k)}(t)\right]^{2} d t=1
$$

The composite transmitted CDMA signal is defined as $[35,36]$

$$
\begin{aligned}
s(t) & =\sum_{k=1}^{K} s_{k}(t) \\
& =\sum_{k=1}^{K} \sqrt{E_{k}} \sum_{n=-\infty}^{\infty} a_{n}^{(k)} c^{(k)}\left(t-n T-\tau^{(k)}\right) .
\end{aligned}
$$




\subsubsection{Receiver system}

In synchronous transmission, each user produces exactly one symbol that interferes with the desired $k$ th user symbol. Therefore, it is sufficient to consider only one signaling interval, that is

$$
s(t)=\sum_{k=1}^{K} \sqrt{E_{k}} a_{1}^{(k)} c^{(k)}(t), \quad 0 \leq t \leq T
$$

In asynchronous transmission, there are exactly two consecutive symbols from each user that overlap a desired $k$ th user symbol [36]. Therefore, it is convenient to consider the transmission of a block of symbols of some arbitrary length $N$, that is

$$
s(t)=\sum_{k=1}^{K} \sqrt{E_{k}} \sum_{n=1}^{N} a_{n}^{(k)} c^{(k)}\left(t-n T-\tau^{(k)}\right) .
$$

The transmitted signal is assumed to be corrupted by AWGN $w(t)$ with PSD of $N_{0} / 2$. Hence, the received signal will have the form

$$
u(t)=s(t)+w(t)
$$

In the following, we briefly discuss two types of receivers: the well known theoretical optimum receiver and the widely used sub-optimum decorrelating receiver. Further details can be found in $[36,37]$. 


\section{Optimum receiver}

The optimum receiver selects the sequence of symbols $\left\{a_{n}^{(k)}, 1 \leq k \leq K\right\}$ for synchronous transmission, or $\left\{a_{n}^{(k)}, 1 \leq n \leq N, 1 \leq k \leq K\right\}$ for asynchronous transmission, that minimizes the log-likelihood function

$$
\Lambda(\mathrm{al})=\int_{D(t)}(u(t)-s(t))^{2} d t
$$

where $D(t)=\{t: 0 \leq t \leq T\}$ and $D(t)=\{t: 0 \leq t \leq(N+2) T\}$ are the domain of integration for synchronous and asynchronous transmission, respectively. As it sufficiently serves the goal of this dissertation, we shall consider the case of synchronous transmission, where the log-likelihood function can be expressed in the form of correlation metrics as $[36 ;$ p. 907,$37 ;$ p. 162]

$$
C\left(\text { un }^{(K)} \text {, al }{ }^{(K)}\right)=2 \sum_{k=1}^{K} \sqrt{E_{k}} a_{1}^{(k)} u^{(k)}-\sum_{j=1}^{K} \sum_{k=1}^{K} \sqrt{E_{j} E_{k}} a_{1}^{(j)} a_{1}^{(k)} \rho_{j k}(0)
$$

where

$$
u^{(k)}=\int_{0}^{T} u(t) c^{(k)}(t) d t, \quad 1 \leq k \leq K
$$




$$
\mathrm{a}^{(K)}=\left[\sqrt{E_{1}} a_{1}^{(1)} \cdots \sqrt{E_{K}} a_{1}^{(K)}\right]^{T}
$$

and

$$
\mathbb{w n}^{(K)}=\left[\begin{array}{llll}
u^{(1)} & u^{(2)} & \cdots & u^{(K)}
\end{array}\right]^{T} .
$$

Therefore, the optimum receiver for synchronous transmission consists of a bank of $K$ correlators or $K$ filters matched to the spreading waveform $c^{(k)}(t)$ followed by a detector, which selects the sequence that has the maximum among $2^{K}$ correlation metrics corresponding to the $2^{K}$ possible transmitted information sequences. The block diagram of the optimum receiver is illustrated in Fig. 2.5.

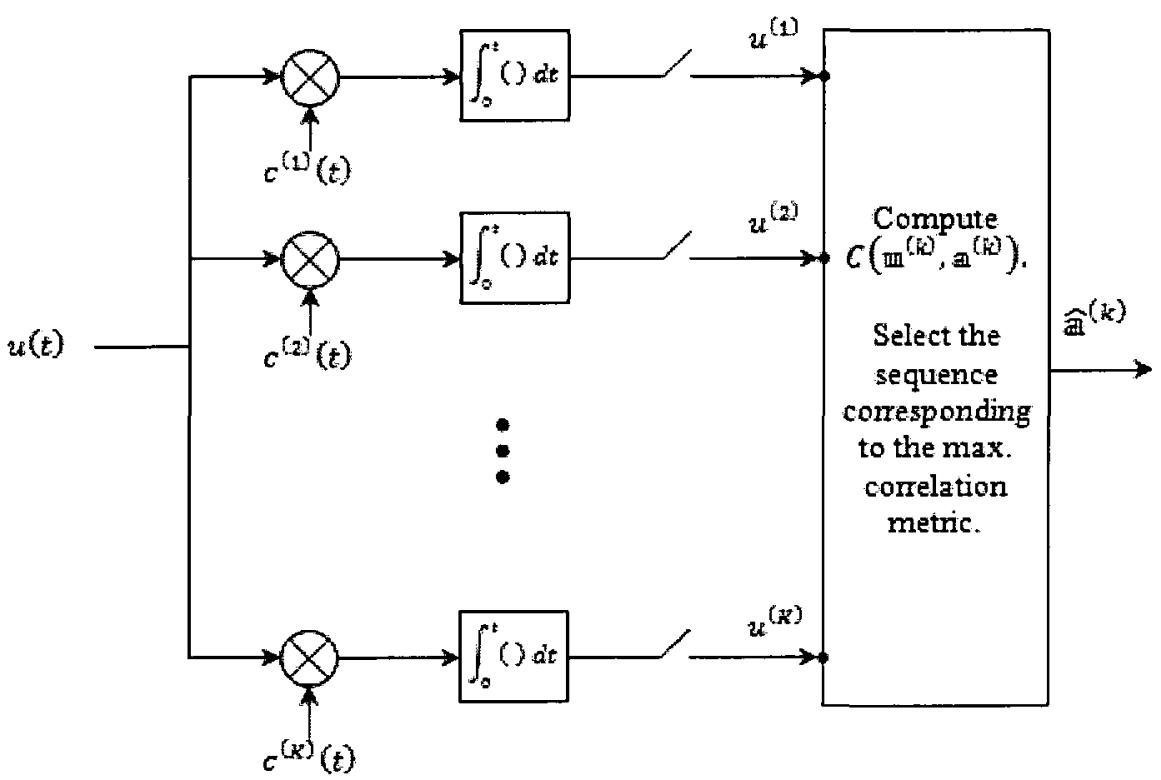

Fig. 2.5 Optimum receiver for synchronous transmission. [36] 
Such an optimum receiver for $K$ CDMA users is very difficult to implement in practice because of its high computational complexity that grows exponentially with $K$.

\section{Suboptimum receivers}

In order to counteract the computational complexity in optimum receivers, suboptimum receivers whose computational complexities grow linearly with the number of users, $K$, were introduced. Consider the conventional single-user detection in which each user's receiver consists of a demodulator that correlates (matched-filters) the received signal with the corresponding spreading waveform. The correlator output is passed to the detector to make a decision. For synchronous transmission, the correlator output for the $k$ th user in the interval $0 \leq t \leq T$ is

$$
\begin{aligned}
u^{(k)} & =\int_{0}^{T} u(t) c^{(k)}(t) d t \\
& =\sqrt{E_{k}} a_{1}^{(k)}+\sum_{\substack{j=1 \\
j \neq k}}^{K} \sqrt{E_{j}} a_{1}^{(j)} \rho_{j k}(0)+w_{1}^{(k)}
\end{aligned}
$$

where $w_{1}^{(k)}$ is the noise component with variance $\sigma_{w}^{2}=N_{0} / 2$, is given by

$$
w_{1}^{(k)}=\int_{0}^{T} w(t) c^{(k)}(t) d t
$$


The middle term in (2.33) represents the interference from other users on the $k$ th user. This term clearly vanishes if the spreading waveforms are orthogonal, otherwise this interference can be excessive, especially if one or more of the other users have significantly larger power than that of the $k$ th user. This situation is called the near-far problem in multiuser communications, which necessitates some type of power control [36]. In asynchronous transmission, other user's interference is unavoidable even with orthogonal spreading waveforms. This is because orthogonality cannot be achieved for all time offsets. Consequently, the near-far problem resulting from unequal powers of other users' signals is particularly serious.

Another type of suboptimum receivers that has been widely used is the decorrelating receiver, which also has a linear computational complexity. The advantage of the decorrelating receiver is that the multiuser interference has been eliminated. For synchronous transmission, the output of the $K$ matched filters is

$$
\mathbb{u d}^{(K)}=\mathbb{R}_{S} \mathbb{a l}^{(K)}+\mathbb{m}^{(K)}
$$

where $\mathbb{R}_{S}$ is the correlation matrix with elements $\rho_{j k}(0)$ and $\mathrm{m}^{(K)}=$ $\left[\begin{array}{llll}n_{1}^{(1)} & n_{1}^{(2)} & \cdots & n_{1}^{(K)}\end{array}\right]^{T}$ is the noise vector with a covariance

$$
E\left\{\mathbb{m}^{(K)} \mathbb{m}^{(K)^{T}}\right\}=\frac{N_{0}}{2} \mathbb{R}_{s}=\sigma_{w}^{2} \mathbb{R}_{s}
$$


Consequently, because the noise is Gaussian, $\mathbb{u}^{(K)}$ is described by a $K$-dimensional Gaussian pdf with mean $\mathbb{R}_{s}$ all ${ }^{(K)}$ and covariance $\mathbb{R}_{s}$. That is

$$
f\left(\text { un }^{(K)}\right)=\frac{1}{\sqrt{\left(\pi N_{0}\right)^{K}\left|\mathbb{R}_{s}\right|}} \exp \left(-\frac{1}{N_{0}} \Lambda\left(\text { aी }^{(K)}\right)\right)
$$

where

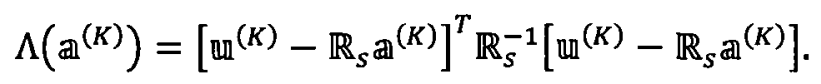

The best linear estimate of $\mathrm{al}^{(K)}$ is $\mathrm{all}_{0}^{(k)}=\mathbb{R}_{s}^{-1} \mathrm{an}^{(K)}$ that minimizes $\Lambda\left(\mathrm{al}^{(K)}\right)$. Ultimately, the detected symbols are determined by taking the sign of each element of $a_{0}^{(k)}$, i.e.,

$$
\widehat{\mathrm{a}}^{(k)}=\operatorname{sgn}\left(\mathrm{a}_{0}^{(k)}\right) \text {. }
$$

the block diagram of the decorrelating receiver is depicted in Fig. 2.6. 


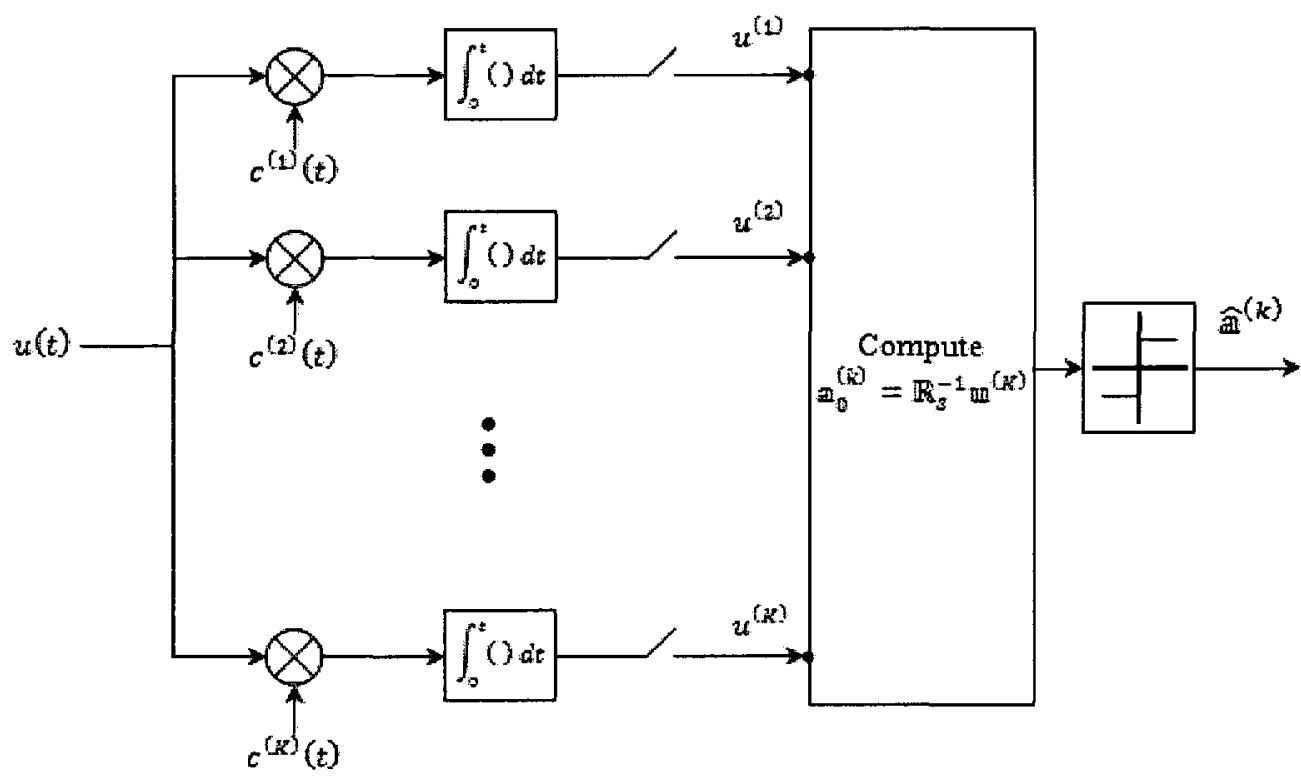

Fig. 2.6 Decorrelating receiver [36]

\subsubsection{OFDM basics}

OFDM is a special case of multicarrier transmission, which uses parallel data transmission and frequency division multiplexing (FDM). Such a concept dates back to the mid 1960's, when Chang published a paper on the synthesis of bandlimited signals for multichannel transmission [38]. He presents a principle for transmitting messages simultaneously through a linear bandlimited channel without interchannel interference (ICI) and ISI. A major contribution to OFDM was presented in 1971 by Weinstein and Ebert [39], who used the discrete Fourier transform (DFT) to perform baseband modulation and demodulation instead of the banks of subcarrier oscillators. To combat 
ISI and ICI, they used both a guard space between the symbols and raised cosine windowing in the time domain. Another important contribution was due to Peled and Ruiz in 1980 [40], who introduced the cyclic prefix (CP) to ensure perfect orthogonality. Instead of using an empty guard space, they filled the guard space with a cyclic extension of the OFDM symbol.

Nowadays, OFDM has become a key element of many modern communication systems since it fits with the increased and continuous demands of higher data rates, quality of service, and capacity. Additionally, it is spectral efficient and robust against multipath fading. Accordingly, OFDM has been selected for many applications such as: cable access networks (asymmetric digital subscriber line (ADSL), high bit rate digital subscriber line (HDSL)), digital audio/video broadcasting (DAB/DVB), wireless local area networks (LANs) (802.11, high performance radio LAN (HiperLAN)), worldwide interoperability for microwave access (WiMAX, 802.16) [41, 42, 43]. In the following, we briefly shed light on the basic concepts of the OFDM system.

\subsubsection{Transmitter system}

Let $A_{k}, k=1, \ldots, N$ denote the complex symbols to be transmitted by an OFDM system. At the OFDM transmitter, the serial bit stream with a high rate $R$ is split into $N$ parallel substreams, $k=1, \ldots, N$, each transmitting at a lower bit rate of $R / N$. After 
symbol mapping, the set of $N$ parallel complex symbols $\left\{A_{k}\right\}$ modulate $N$ subcarrier waveforms, when summed results in the following OFDM block (frame) [41, 44]

$$
s(t)=\frac{1}{N} \sum_{k=1}^{N} A_{k} \varphi_{k}(t), \quad 0 \leq t \leq T
$$

where $T$ is the symbol duration and $\varphi_{k}(t)$ is the subcarrier waveform given by

$$
\varphi_{k}(t)=\left\{\begin{aligned}
e^{j 2 \pi f_{k}\left(t-T_{g}\right)}, & 0 \leq t \leq T \\
0, & \text { otherwise }
\end{aligned}\right.
$$

where $f_{k}=k \Delta f, \Delta f$ is the subcarrier spacing, and $T_{g}$ is the guard time. To guarantee that the OFDM signal can be demodulated properly by the receiver, the orthogonality condition must be satisfied. The orthogonality condition is $\Delta f=1 / T_{e}$ with $T_{e}$ is the effective symbol time $T_{e}=T-T_{g}$, where it can be shown that

$$
\begin{aligned}
\frac{1}{T} \int_{0}^{T} \varphi_{k}(t) \varphi_{l}^{*}(t) d t & =\frac{1}{T} \int_{0}^{T} e^{j 2 \pi\left(f_{k}-f_{l}\right)\left(t-T_{g}\right)} d t \\
& =e^{j 2 \pi(k-l) \Delta f T_{g}} \frac{1}{T} \int_{0}^{T} e^{j 2 \pi(k-l) \Delta f t} d t \\
& =e^{j 2 \pi(k-l) \Delta f T_{g}} \delta[k-l],
\end{aligned}
$$

where $\delta[k-l]$ is the delta function defined as 


$$
\delta[n]= \begin{cases}1, & n=0 \\ 0, & \text { otherwise }\end{cases}
$$

With $N$ subcarriers, the total system bandwidth is $B=N \Delta f$. The guard time $T_{g}$ is usually filled with a copy of the symbol tail end to eliminate ICI, which is referred to as cyclic prefix. It is worth mentioning that in many OFDM systems with a large number of subcarriers, pulse shaping is not usually employed, since the PSD of the band-limited OFDM signal is approximately rectangular [21, 45].

It has been shown that the process of OFDM modulation can be realized via inverse DFT (IDFT), usually implemented by the inverse fast Fourier transform (IFFT). By sampling the OFDM symbol of (2.41) at an interval $T / N$, the sampled OFDM symbol becomes

$$
\begin{aligned}
s_{n} & \left.\triangleq s(t)\right|_{t=\frac{n T}{N}}=\frac{1}{N} \sum_{k=1}^{N} A_{k} e^{\frac{j 2 \pi n k}{N}} \\
& =\operatorname{IDFT}\left\{A_{k}\right\}, \quad n=1, \ldots, N .
\end{aligned}
$$

The block diagrams of the baseband OFDM transmitter and its discrete model using IFFT are illustrated in Fig. 2.7 and Fig. 2.8, respectively, where $h(t)$ in Fig. 2.8 represents the waveform adopted for digital to analog conversion and its frequency response is $H(f)$. 


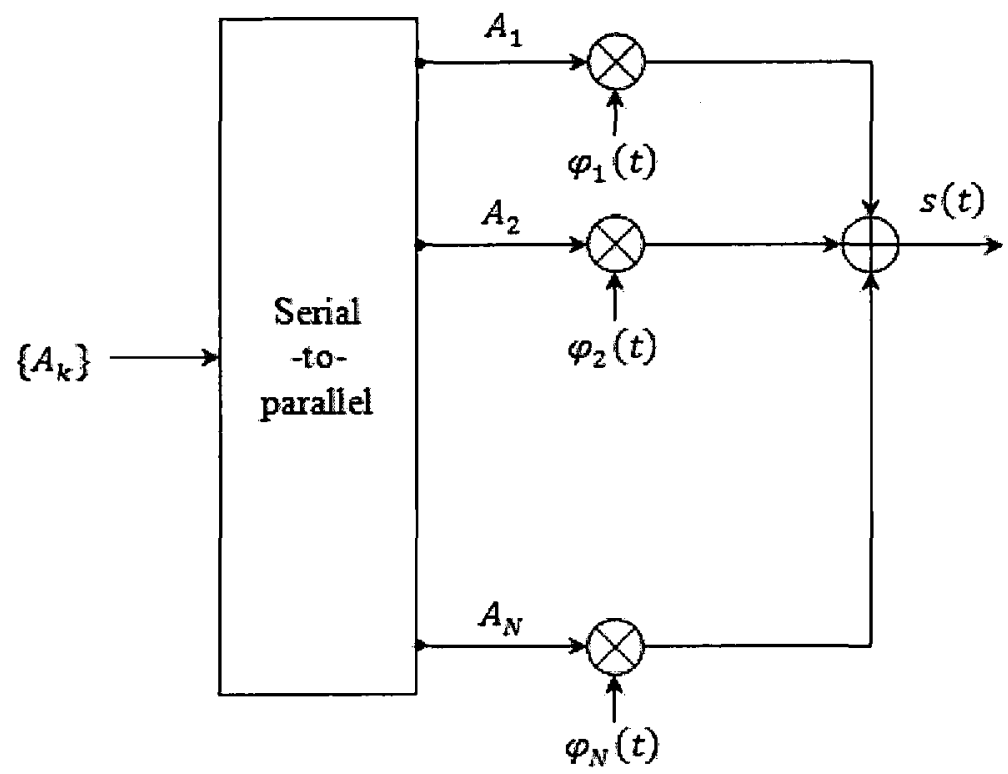

Fig. 2.7 Baseband block diagram of the OFDM transmitter [41].

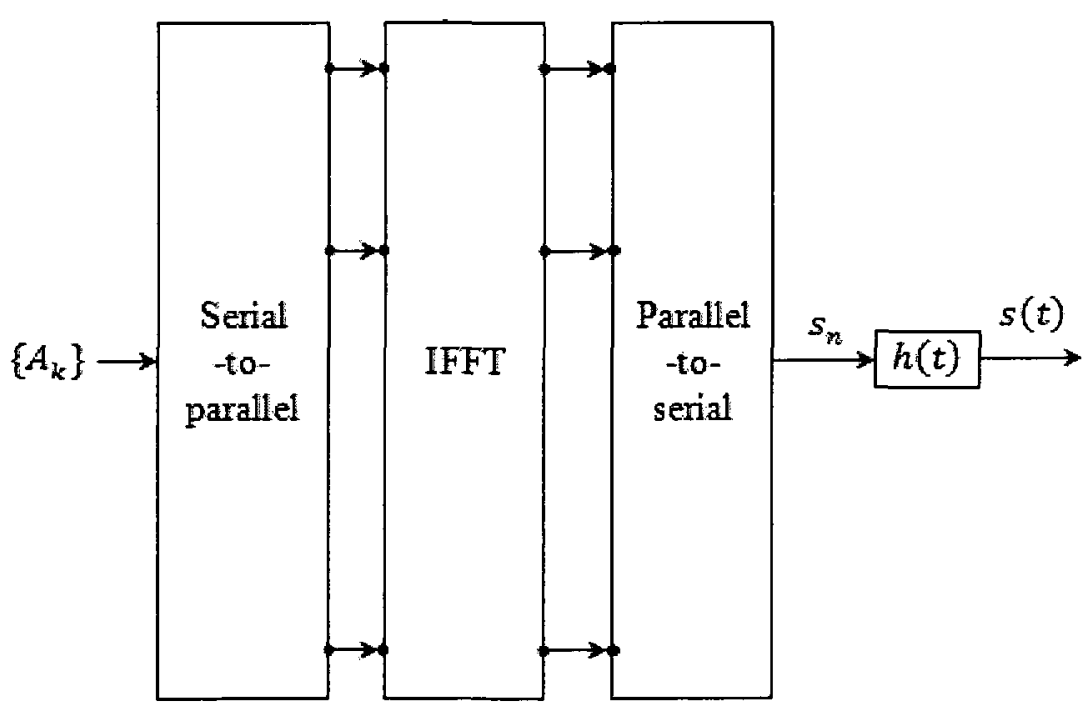

Fig. 2.8 Baseband block diagram of the OFDM transmitter using IFFT [41, 44]. 


\subsubsection{Receiver system}

At the receiver, the OFDM signal perturbed by a zero-mean AWGN $w(t)$, as expressed by $u(t)=s(t)+w(t)$ is filtered by the receiver filter $g(t)$, whose frequency response is $G(f)$. The filtered signal $v(t)$ is sampled at instants $n T$, obtaining $v_{n}=s_{n}+$ $w_{n}$. The sampled sequence is processed, frame by frame, by the FFT block giving

$$
V_{k}=\sum_{n=1}^{N} v_{n} e^{-\frac{j 2 \pi n k}{N}}=A_{k}+W_{k}
$$

where

$$
W_{k}=\sum_{n=1}^{N} w_{n} e^{-\frac{j 2 \pi n k}{N}}
$$

The discrete model of the baseband OFDM transmitter using FFT is illustrated in Fig. 2.9. 


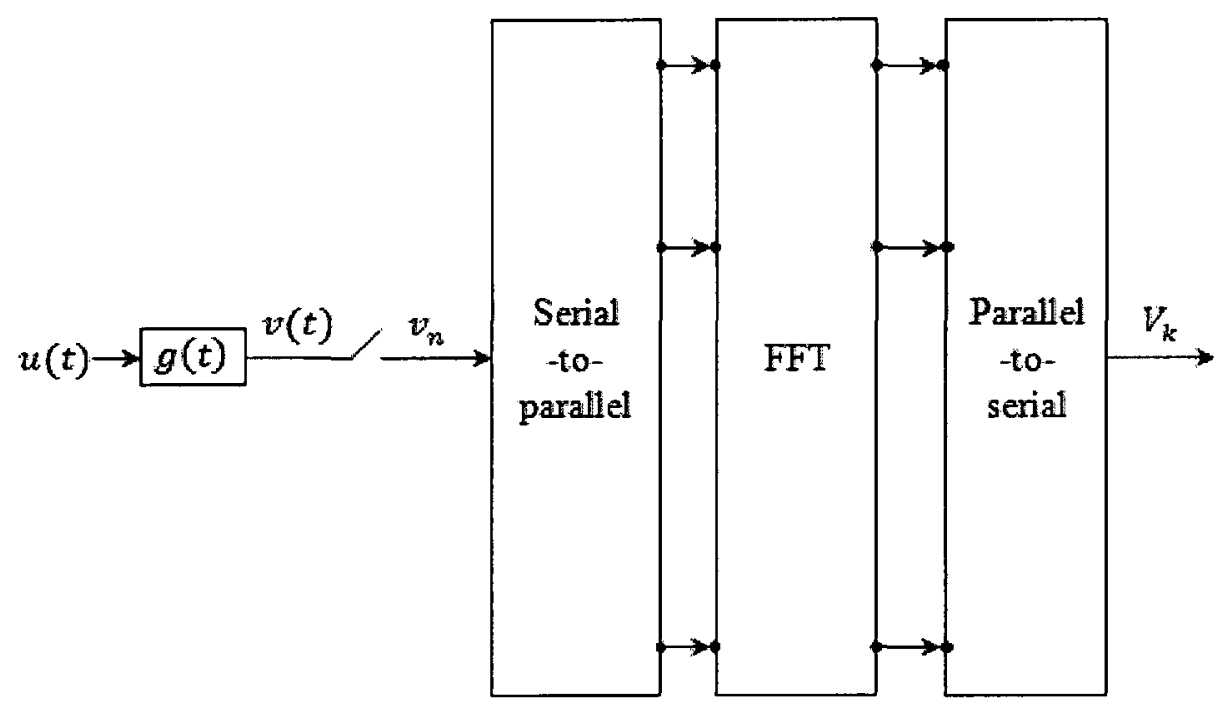

Fig. 2.9 Baseband block diagram of the OFDM receiver using FFT [41, 44]. 


\section{Chapter 3}

\section{ANALYSIS OF NONLINEAR DISTORTION EFFECTS ON BER PERFORMANCE}

B it error rate (BER) is one of the most desirable performance merits in wireless communication systems. Signals characterized by large dynamic range, when passed through an HPA, results in distortion for components falling in the highly nonlinear regions of the HPA characteristics, leading to degradation in the system BER. In this chapter, we analyze the BER performance to explore which characteristics of the input signal to the HPA, in relation to the HPA characteristics, contribute to BER degradation.

Two signal types that are widely used in modern communications systems, because of their many advantages, are investigated; CDMA and OFDM. Unfortunately, such signals typically exhibit large dynamic range, which makes them more susceptible to nonlinear distortion due to an HPA. In our analysis, we consider the AWGN as the channel impairment, where such an assumption sufficiently serves the goal of the dissertation. Also, the PD-HPA pair is considered as the nonlinear amplifier, which represents the most cost-effective technique among many HPA linearization techniques $[46,47]$ and has become a common practice in wireless communication to compensate for nonlinear distortion due to an HPA [5]. The PD-HPA has an AM-AM characteristic 
that is linear up to the HPA saturation level $\zeta$, after which the output signal is clipped, and a zero AM-PM characteristic [48], that is

$$
\begin{gathered}
r_{d}(t)=G[r(t)]=\left\{\begin{array}{lr}
r(t) & 0 \leq r(t) \leq \zeta \\
\zeta & r(t)>\zeta^{\prime}
\end{array}\right. \\
\Phi[r(t)]=0
\end{gathered}
$$

where $r(t)=|s(t)|$ is the signal envelope input to the PD-HPA.

In the analysis, we investigate the SNR including the nonlinear distortion noise at the receiver input, since it represents the pivoting parameter in the BER performance of all types of receivers depending on the adopted modulation format. That is

$$
\mathrm{BER}=f(\text { modulation, } \mathrm{SNR})
$$

Table 3.1 summarizes the BER performances of some of the most common modulation formats as functions of the SNR per bit and the number of modulation levels $M[36,49]$, in terms of the $Q$ function that represents the area under the tail of the Gaussian probability density function (pdf), i.e.,

$$
Q(x)=\frac{1}{\sqrt{2 \pi}} \int_{x}^{\infty} \exp \left(\frac{-u^{2}}{2}\right) d u
$$


Table 3.1 BER performance of different modulation formats.

\begin{tabular}{|c|c|}
\hline BER & Modulation format \\
\hline$Q(\sqrt{\mathrm{SNR}})$ & Coherent BPSK \\
\hline$Q(\sqrt{\mathrm{SNR}})$ & Coherent QPSK \\
\hline $2 Q\left(\sqrt{\mathrm{SNR}} \cdot \sin \left(\frac{\pi}{M}\right)\right)$ & Coherent MPSK \\
\hline$(M-1) Q(\sqrt{\mathrm{SNR} / 2})$ & Coherent MFSK \\
\hline $0.5 \exp (-\operatorname{SNR})$ & Non-coherent DPSK \\
\hline $0.5(M-1) \exp (-\mathrm{SNR} / 2)$ & Non-coherent MFSK \\
\hline$Q\left(\sqrt{2 \mathrm{SNR}} \cdot \sin \left(\frac{\pi}{2 M}\right)\right)$ & Non-coherent MPSK \\
\hline$\frac{4}{\log _{2} M}\left(1-\frac{1}{\sqrt{M}}\right) Q\left(\sqrt{\frac{3}{M-1} S N R}\right)$ & Coherent MQAM \\
\hline
\end{tabular}

\subsection{BER performance analysis for CDMA signals}

Consider the synchronous transmission of the CDMA signal in (2.25), where users' signals have equal power and the symbols $a_{n}^{(k)}$ are assumed equiprobable, statistically independent identically distributed (i.i.d.) with zero mean and variance of $E\left\{\left|a_{n}^{(k)}\right|^{2}\right\}$. Also, symbols $a_{n}^{(k)} \in\left\{\chi_{i}\right\}_{i=1, \ldots, M}$, where $\chi_{i} \in\left\{ \pm \alpha_{i} \pm j \beta_{i}\right\}$ with $\alpha_{i}, \beta_{i} \in \mathbb{R}$. In our analysis, we consider QPSK modulation with $\chi_{i} \in\{ \pm 1 \pm j\} / \sqrt{P}$ and 16QAM with $\chi_{i} \in\{ \pm 1 \pm j, \pm 1 \pm j 3, \pm 3 \pm j, \pm 3 \pm j 3\} / \sqrt{P}$, where $P=2(M-1) / 3$. Extensions to 
other modulation formats are straightforward. Therefore, the CDMA signal $s(t)$ in (2.24) for one symbol duration can have the form

$$
\begin{aligned}
s(t) & =\sum_{k=1}^{K} \sqrt{E_{s}} a_{n}^{(k)} c^{(k)}(t), \quad(n-1) T \leq t \leq n T \\
& =x(t)+j y(t)=r(t) e^{j \theta(t)},
\end{aligned}
$$

where $r(t)=|s(t)|=\sqrt{x^{2}(t)+y^{2}(t)}$ and $\theta(t)=\angle s(t)$ are the envelope and phase of $s(t)$, respectively.

For large number of users and assuming the pulse shaping filter corresponds to a square root raised cosine (SRRC) filter with small roll-off factor, $x(t)$ and $y(t)$ can be regarded as two uncorrelated zero-mean Gaussian random processes with equal variances, $\sigma_{x}^{2}=\sigma_{y}^{2}$, because of the Central Limit Theorem (CLT) $[35,50]$. Consequently, $s(t)$ can be regarded as a band-limited zero-mean complex Gaussian process with variance

$$
\sigma_{s}^{2}=2 \sigma_{x}^{2}=E_{s} \sum_{k=1}^{K} E\left\{\left|a_{n}^{(k)}\right|^{2}\right\}=K E_{s}
$$

since $\left|c_{l}^{(k)}\right|=1$, and its envelope $r(t)$ has a quasi-Rayleigh distribution [50], whose pdf is given by 


$$
f_{r}(r)=\frac{r}{\sigma_{x}^{2}} \exp \left(-\frac{r^{2}}{2 \sigma_{x}^{2}}\right), r \geq 0
$$

with mean $\mu_{r}=\sqrt{\pi \sigma_{x}^{2} / 2}$ and variance $\sigma_{r}^{2}=(4-\pi) \sigma_{x}^{2} / 2[36]$.

In presence of the PD-HPA, whose characteristics are given in (3.1), its output can be expressed as [48]

$$
\begin{aligned}
s_{d}(t) & =r_{d}(t) e^{j(\theta(t)+\Phi[r(t)])} \\
& =\left(r(t)-r_{c}(t)\right) e^{j \theta(t)} \\
& =s(t)-s_{c}(t)
\end{aligned}
$$

where $s_{c}(t)=r_{c}(t) e^{j \theta(t)}$ is the signal portion that is clipped from the input signal $s(t)$ by the PD-HPA, $E\left\{s_{c}(t)\right\}=0$, and its envelope $r_{c}(t)$ has the form

$$
\begin{aligned}
r_{c}(t) & =r(t)-r_{d}(t) \\
& =\left\{\begin{array}{ll}
0, & r(t) \leq \zeta \\
r(t)-\zeta, & r(t)>\zeta
\end{array} .\right.
\end{aligned}
$$

Examples of the input signal envelope $r(t)$ to the PD-HPA, the corresponding envelope of the output signal $r_{d}(t)$ of the PD-HPA, and the envelope of the clipped signal portion $r_{c}(t)$ are shown in Fig. 3.1(a), Fig. 3.1(b), and Fig. 3.1(c), respectively. 


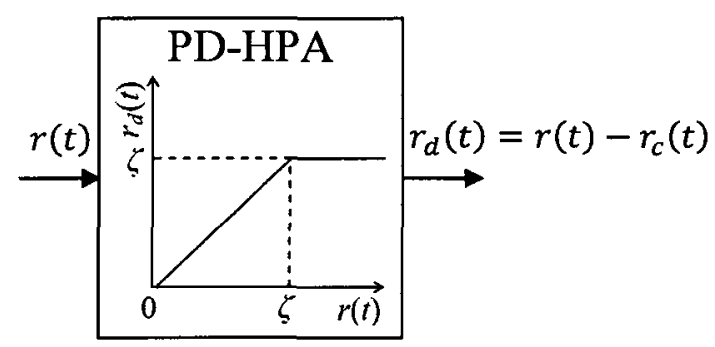

Fig. 3.1 PD-HPA transfer function, (a) envelope of the input signal to PD-HPA $r(t)$, (b) envelope of the corresponding output signal $r_{d}(t)$ from PD-HPA, and (c) envelope of the clipped signal portion $r_{c}(t)$.

Using the Bussgang theorem extension for a complex Gaussian input to a memoryless nonlinear device, the output of the PD-HPA can be represented as the sum of two uncorrelated components: a scaled linear component and a nonlinear component, $n(t),[35,50,51,52]$, that is 


$$
s_{d}(t)=\alpha_{0} s(t)+n(t)
$$

where $E\{n(t)\}=0, E\left\{s(t) n^{*}(t+\tau)\right\}=0, \forall t, \tau$, and $\alpha_{0}$ is the linear gain of the amplifier given by

$$
\begin{aligned}
\alpha_{0} & =\frac{E\left\{s_{d}^{*}(t) s(t)\right\}}{E\left\{|s(t)|^{2}\right\}}=\frac{E\left\{r_{d}(t) r(t)\right\}}{E\left\{r^{2}(t)\right\}} \\
& =\frac{1}{2 \sigma_{x}^{2}}\left(\int_{0}^{\zeta} r^{2} f_{r}(r) d r+\zeta \int_{\zeta}^{\infty} r f_{r}(r) d r\right) \\
& =1-\exp \left(-\frac{\zeta^{2}}{2 \sigma_{x}^{2}}\right)+\frac{1}{2} \sqrt{\frac{\pi \zeta^{2}}{2 \sigma_{x}^{2}}} \operatorname{erfc}\left(\sqrt{\frac{\zeta^{2}}{2 \sigma_{x}^{2}}}\right)
\end{aligned}
$$

Consequently, the variance of the distorted output signal $s_{d}(t)$ is given by

$$
\sigma_{d}^{2}=\left|\alpha_{0}\right|^{2} \sigma_{s}^{2}+\sigma_{n}^{2}
$$

where $\sigma_{n}^{2}$ is the variance of the nonlinear component $n(t)$. As far as $s(t)$ is considered as a zero-mean complex Gaussian process and using the transfer function of the considered nonlinear PD-HPA in (3.1), the variance of the distorted output signal $\sigma_{d}^{2}$ can be calculated as

$$
\sigma_{d}^{2}=E\left\{\left|s_{d}(t)\right|^{2}\right\}=E\left\{r_{d}^{2}(t)\right\}
$$




$$
\begin{aligned}
& =\int_{0}^{\zeta} r^{2} f_{r}(r) d r+\int_{\zeta}^{\infty} \zeta^{2} f_{r}(r) d r \\
& =2 \sigma_{x}^{2}\left(1-\exp \left(-\frac{\zeta^{2}}{2 \sigma_{x}^{2}}\right)\right) \\
& =\sigma_{s}^{2}\left(1-\exp \left(-\frac{\zeta^{2}}{2 \sigma_{x}^{2}}\right)\right) .
\end{aligned}
$$

At the $k$ th user's receiver, complex zero-mean AWGN $w(t)$ with power spectral density of $N_{0} / 2$ is introduced and the received signal is expressed as

$$
\begin{aligned}
u(t) & =s_{d}(t)+w(t) \\
& =\alpha_{0} s(t)+n(t)+w(t) .
\end{aligned}
$$

Assuming perfect synchronization, after coherent demodulation and phase recovery, the received signal is match-filtered with the $k$ th user's spreading waveform $c^{(k)}(t)$ and passed to the detector. Thus, for the signal in the interval $(n-1) T \leq t \leq n T$, the input to the detector of the $k$ th user, with setting $n=1$ without loss of generality, is given by

$$
\begin{aligned}
u_{1}^{(k)} & =\int_{0}^{T} u(t) c^{(k)}(t) d t \\
& =\alpha_{0}\left(s_{1}^{(k)}+\mathrm{MAI}\right)+n_{1}^{(k)}+w_{1}^{(k)},
\end{aligned}
$$


where

$$
s_{1}^{(k)}=\sqrt{E_{s}} a_{1}^{(k)} \int_{0}^{T}\left[c^{(k)}(t)\right]^{2} d t=\sqrt{E_{s}} a_{1}^{(k)}
$$

and

$$
\begin{aligned}
\mathrm{MAI} & =\sum_{\substack{j=1 \\
j \neq k}}^{K} \sqrt{E_{s}} a_{1}^{(j)} \int_{0}^{T} c^{(j)}(t) c^{(k)}(t) d t \\
& =\sum_{\substack{j=1 \\
j \neq k}}^{K} \sqrt{E_{s}} a_{1}^{(j)} \rho_{j k}(0)
\end{aligned}
$$

is the multiple access interference (MAI) component due to users $j \neq k$, which vanishes for CDMA with orthogonal codes. For nonorthogonal CDMA, MAI can be assumed a Gaussian distributed random variable when the number of users $K$ and spreading factor $L$ are sufficiently large [35]. The variance of the MAI for synchronous CDMA can be easily evaluated $[53,54]$, leading to

$$
\sigma_{\mathrm{MAI}}^{2}=\frac{\sigma_{s}^{2}}{L} \frac{K-1}{K}
$$

In (3.14),

$$
n_{1}^{(k)}=\int_{0}^{T} n(t) c^{(k)}(t) d t
$$


is the nonlinear component due to the nonlinear device and

$$
w_{1}^{(k)}=\int_{0}^{T} w(t) c^{(k)}(t) d t
$$

is the additive Gaussian noise component with variance $\sigma_{W}^{2}=E\left\{\left|n_{1}^{(k)}\right|^{2}\right\}=$ $\left(N_{0} / 2\right) \int_{0}^{T}\left[c^{(k)}(t)\right]^{2} d t=N_{0} / 2[36$, eq. $(15.3-29)]$

Evaluating the BER in the case of an optimum receiver is extremely difficult and tedious. Hence, in the presence of AWGN only, the BER for a single-user receiver in the absence of other users in the channel can be used as a lower bound, where the SNR per bit in this case is given by

$$
\mathrm{SNR}_{k}=\frac{2 E_{b}}{N_{0}}
$$

where $E_{b}$ is the $k$ th user's signal energy per bit. The BER for a suboptimum receiver can be used as an upper bound [36]. In our analysis, we adopt one of the well known suboptimum receivers; the decorrelating receiver, where the other-user interference is completely eliminated at the expense of noise enhancement $[36,51]$. For the decorrelating receiver, in presence of AWGN only (without nonlinearity), the $\mathrm{SNR}_{k}$ at the $k$ th user's receiver is given by [36, eq. $(15.3-67)]$

$$
\mathrm{SNR}_{k}=\mathrm{SNR}_{\mathrm{AWGN}, k}=\frac{\sigma_{s, k}^{2} / \log _{2} M}{\sigma_{W, k}^{2}}=\frac{2 E_{b}}{\varepsilon_{k} N_{0}}
$$


where $\mathrm{SNR}_{\mathrm{AWGN}}$ is the SNR per bit due to the AWGN only and $\varepsilon_{k}=\left(\mathbb{R}_{s}^{-1}\right)_{k, k} \geq 1$ is the noise enhancement factor introduced by the decorrelating receiver [36], where $\mathbb{R}_{s}$ is the correlation matrix with elements $\rho_{j k}(0)=\int_{0}^{T} c^{(i)}(t) c^{(j)}(t) d t$. Clearly, for orthogonal CDMA systems, $\varepsilon_{k}=1$ since $\mathbb{R}_{s}$ becomes an identity matrix.

In the presence of a nonlinear amplifier, the nonlinear distortion noise component $n_{1}^{(k)}$ and the AWGN component $w_{1}^{(k)}$ in (3.14) are two uncorrelated noise contributions that distort the received symbols. For large $K$ and consequently large $L$, the nonlinear distortion noise $n_{1}^{(k)}$ can be modeled as a zero-mean Gaussian random process, because of the CLT $[35,51,52]$, with variance

$$
\begin{aligned}
\sigma_{N}^{2} & =\frac{1}{L} E\left\{\left|n_{1}^{(k)}\right|^{2}\right\} \\
& =\frac{1}{L} E\left\{\left|n_{1}^{(k)}\right|^{2}\right\} \int_{0}^{T}\left[c^{(k)}(t)\right]^{2} d t=\frac{\sigma_{n}^{2}}{L} .
\end{aligned}
$$

In this sense, the distortion affects the constellation mapping, in the same way as the Gaussian noise term. As a consequence, the system performance of a CDMA system can be derived, making use of the same relation used for AWGN channels but with redefining the SNR at the detector input taking into account the nonlinear distortion noise contribution. The nonlinear distortion noise is uncorrelated with the thermal noise; they can be added in power and therefore, the SNR at the $k$ th detector input can be defined as 


$$
\begin{aligned}
\mathrm{SNR}_{k} & =\mathrm{SNR}_{\mathrm{NL}, k}=\frac{\left|\alpha_{0}\right|^{2} \sigma_{s, k}^{2} / \log _{2} M}{\sigma_{W, k}^{2}+\sigma_{N, k}^{2} / \log _{2} M} \\
& =\frac{\left|\alpha_{0}\right|^{2} E_{b}}{\varepsilon_{k}\left(\sigma_{W}^{2}+\sigma_{N}^{2} / \log _{2} M\right)} \\
& =\frac{\left|\alpha_{0}\right|^{2} E_{b}}{\varepsilon_{k}\left(\sigma_{W}^{2}+\frac{\sigma_{n}^{2} / L}{\log _{2} M}\right)} \\
& =\frac{\left|\alpha_{0}\right|^{2} E_{b}}{\varepsilon_{k} \frac{N_{0}}{2}+\frac{\varepsilon_{k}}{L \log _{2} M}\left(\sigma_{d}^{2}-\left|\alpha_{0}\right|^{2} \sigma_{s}^{2}\right)} .
\end{aligned}
$$

Let us define two important characteristics of the input signal in relation to the PD-HPA. First, the threshold exceeding rate $R_{E}$ is defined as the total time intervals where the signal exceeds the PD-HPA threshold, or equivalently the probability that the signal exceeds the threshold $\zeta$, as

$$
\begin{aligned}
R_{E} & =P(r>\zeta)=\int_{\zeta}^{\infty} f_{r}(r) d r \\
& =\exp \left(-\frac{\zeta^{2}}{2 \sigma_{x}^{2}}\right) .
\end{aligned}
$$

Second, the variance of the signal portion that exceeds the PD-HPA threshold $s_{c}(t)$, or equivalently the variance of the clipped signal portion $\sigma_{c}^{2}$, which is given as 


$$
\begin{aligned}
\sigma_{c}^{2} & =E\left\{\left|s_{c}(t)\right|^{2}\right\} \\
& =\int_{\zeta}^{\infty}(r-\zeta)^{2} f_{r}(r) d r \\
& =2 \sigma_{x}^{2} \exp \left(-\frac{\zeta^{2}}{2 \sigma_{x}^{2}}\right)-\sqrt{2 \pi \sigma_{x}^{2}} \zeta \operatorname{erfc}\left(\frac{\zeta}{\sqrt{2 \sigma_{x}^{2}}}\right) .
\end{aligned}
$$

Rearranging (3.24) and (3.25), and substituting in (3.10) and (3.12), respectively, gives

$$
\begin{gathered}
\alpha_{0}=1-\frac{R_{E}}{2}-\frac{\sigma_{c}^{2}}{2 \sigma_{s}^{2}}, \\
\sigma_{d}^{2}=\sigma_{s}^{2}\left(1-R_{E}\right) .
\end{gathered}
$$

Substituting (3.26) and (3.27) in the SNR of (3.23), we obtain

$$
\begin{aligned}
\mathrm{SNR}_{\mathrm{NL}, k} & =\frac{\left(1-R_{E} / 2-\sigma_{c}^{2} / 2 \sigma_{s}^{2}\right)^{2} E_{b}}{\varepsilon_{k} \frac{N_{0}}{2}+\frac{\varepsilon_{k}}{L \log _{2} M} \sigma_{s}^{2}\left(1-R_{E}-\left(1-R_{E} / 2-\sigma_{c}^{2} / 2 \sigma_{s}^{2}\right)^{2}\right)} \\
& =\frac{1}{4} \frac{\left(2-R_{E}-\sigma_{c}^{2} / \sigma_{s}^{2}\right)^{2} E_{b}}{\varepsilon_{k} \frac{N_{0}}{2}+\frac{\varepsilon_{k}}{L \log _{2} M} K E_{s}\left(\sigma_{c}^{2} / \sigma_{s}^{2}-\frac{1}{4}\left(R_{E}+\sigma_{c}^{2} / \sigma_{s}^{2}\right)^{2}\right)} \\
& =\frac{1}{4} \frac{\left(2-R_{E}-\sigma_{c}^{2} / \sigma_{s}^{2}\right)^{2}}{\left(\mathrm{SNR}_{\mathrm{AWGN}, k}\right)^{-1}+\frac{K}{L} \varepsilon_{k}\left(\sigma_{c}^{2} / \sigma_{s}^{2}-\frac{1}{4}\left(R_{E}+\sigma_{c}^{2} / \sigma_{s}^{2}\right)^{2}\right)} .
\end{aligned}
$$


It is concluded from (3.28) that the effect of the nonlinear distortion caused by the amplifier can be represented by the signal characteristics $R_{E}$ and $\sigma_{c}^{2}$. This new characterization opens new avenues to minimize the nonlinear distortion via controlling such characteristics as will be demonstrated later.

\subsubsection{Analysis validations and interpretations}

It is more convenient in the context of this dissertation to relate the signal parameters to the IBO level instead of the threshold $\zeta$. Such a relation allows the system operator to determine the appropriate IBO level required to work at, according to the design demands. The IBO is defined as the ratio of the input power at which the HPA start to saturate, $P_{\text {sat }}$, to the signal average power $P_{a v}$. For the considered PD-HPA, the IBO $\gamma$ is defined as

$$
\gamma=\frac{P_{s a t}}{P_{a v}}=\zeta^{2} / \sigma_{s}^{2}=\zeta^{2} / 2 \sigma_{x}^{2}
$$

Hence, the parameters $\alpha_{0}, \sigma_{d}^{2}, R_{E}$, and $\sigma_{c}^{2}$ are, respectively, expressed as

$$
\begin{gathered}
\alpha_{0}=1-\exp (-\gamma)+\frac{1}{2} \sqrt{\pi \gamma} \operatorname{erfc}(\sqrt{\gamma}), \\
\sigma_{d}^{2}=\sigma_{s}^{2}(1-\exp (-\gamma)), \\
R_{E}=\exp (-\gamma),
\end{gathered}
$$


and

$$
\sigma_{c}^{2}=\sigma_{s}^{2}(\exp (-\gamma)-\sqrt{\pi \gamma} \operatorname{erfc}(\sqrt{\gamma}))
$$

To verify the analytic expressions of $\alpha_{0}, \sigma_{d}^{2}, R_{E}$, and $\sigma_{c}^{2}, 8,16$, and 64-user CDMA signals filtered using an SRRC filter with Walsh codes of length $L=64$ are simulated. The signals are normalized such that $\sigma_{s}^{2}=1 . R_{E}, \sigma_{c}^{2}, \alpha_{0}$, and $\sigma_{d}^{2}$ are measured for the simulated signals at different IBO levels and compared against the analytic expressions in (3.30), (3.31), (3.32), and (3.33), respectively. The relations of such factors versus IBO are shown in Fig. 3.2, where the agreement between the analytic curves and simulated points confirms the accuracy of the developed analytic expressions and the assumptions taken during their derivations for $K \geq 8$.

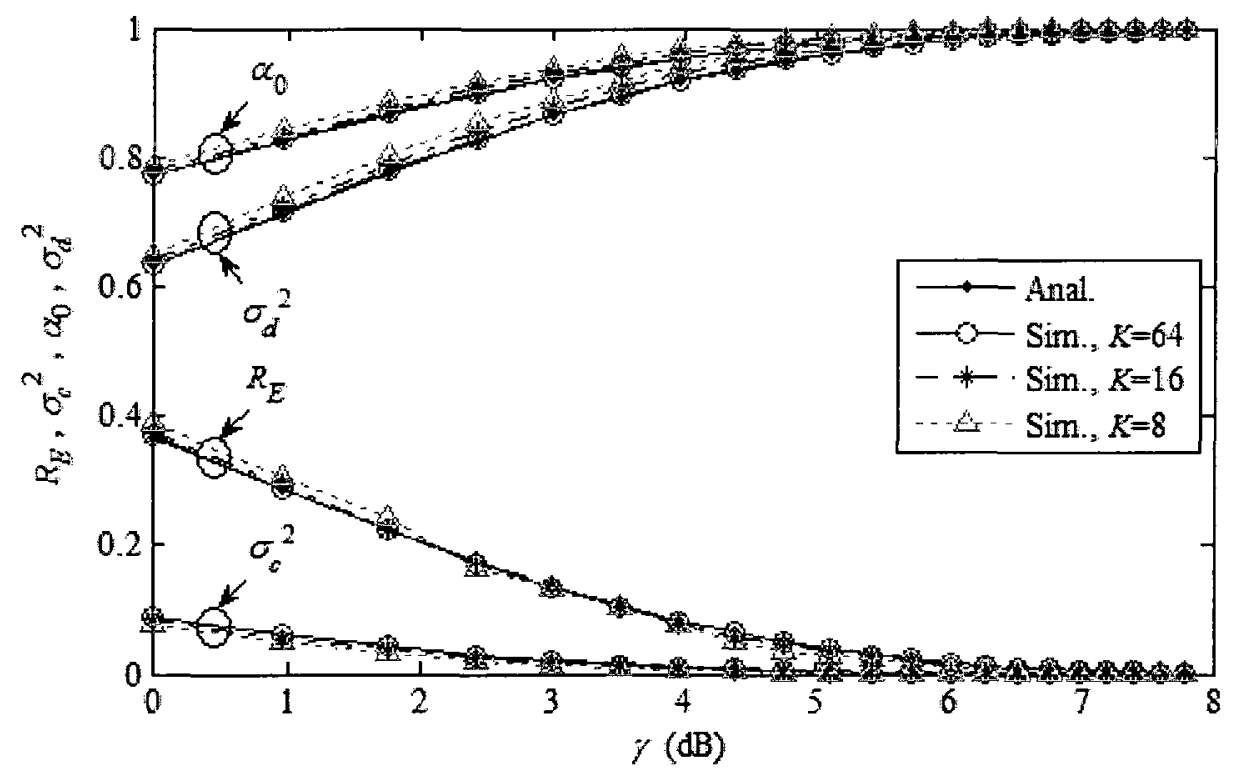

Fig. 3.2 Analytic and simulated plots of $R_{E}, \sigma_{c}^{2}, \alpha_{0}$, and $\sigma_{d}^{2}$ versus IBO levels. 
In order to visualize the impact of the CDMA signal characteristics $R_{E}$ and $\sigma_{c}^{2}$ on the system performance, the SNR in (3.28) is computed at $\mathrm{SNR}_{\mathrm{AWGN}, k}$ of $5 \mathrm{~dB}$ and plotted against $R_{E}$ and $\sigma_{c}^{2}$ in Fig. 3.3. Computations are done at different IBO levels ranging from $0 \mathrm{~dB}$ up to the level that passes almost all of the signal without clipping, i.e., $R_{E} \cong \sigma_{c}^{2} \cong 0$. From (3.28) and Fig. 3.3, it is clear that $R_{E}$ and $\sigma_{c}^{2}$ represent the main contributors to the system performance in presence of the nonlinear distortion due to the PD-HPA; as $R_{E}$ and $\sigma_{c}^{2}$ increase, the overall $\mathrm{SNR}, \mathrm{SNR}_{\mathrm{NL}, k}$, decreases.

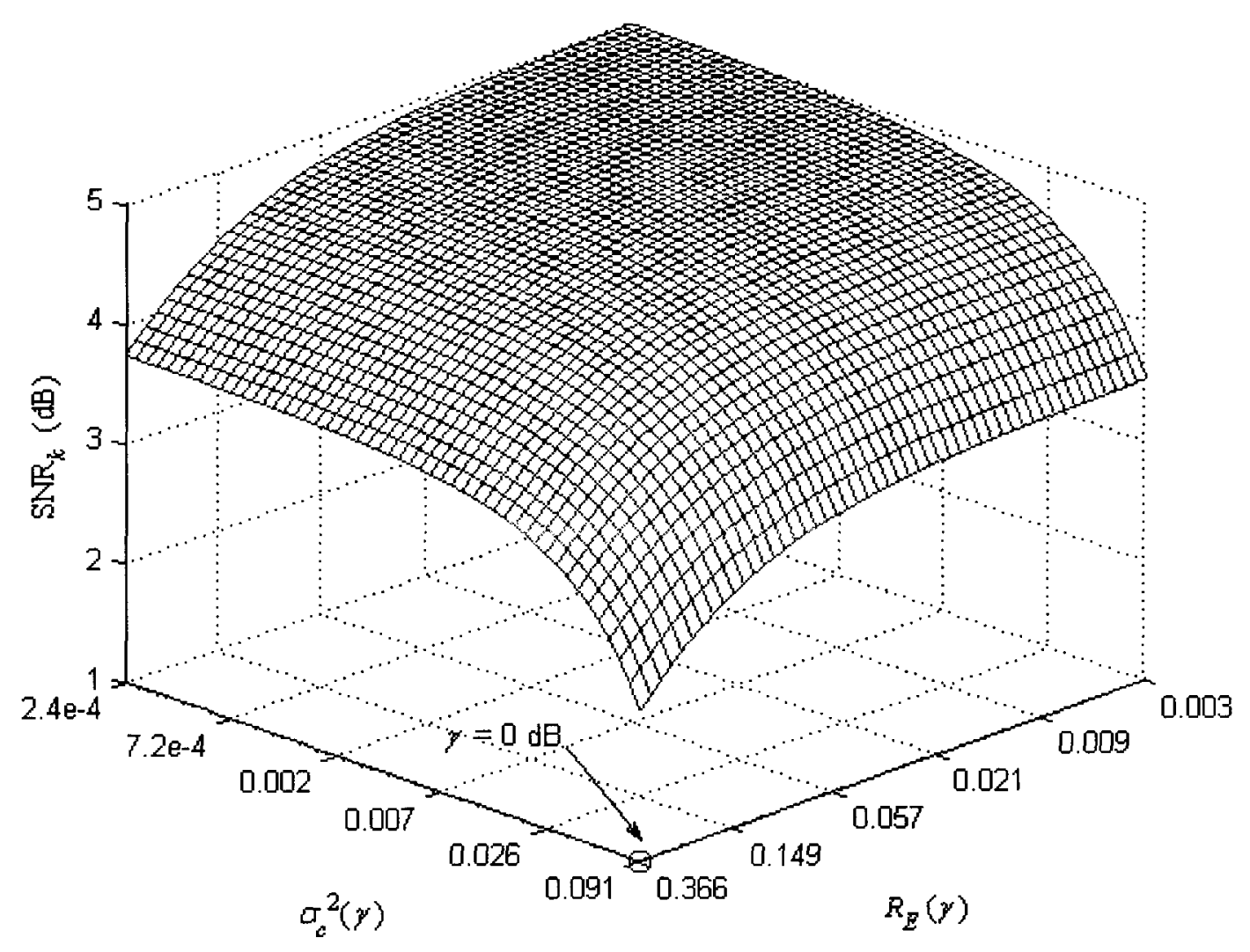

Fig. 3.3 $\mathrm{SNR}_{\mathrm{NL}, k}$ versus $R_{E}$ and $\sigma_{c}^{2}$ at $\mathrm{SNR}_{\mathrm{AWGN}, k}=5 \mathrm{~dB}$. 
Finally, the BER performance using the expressions in Table 3.2 for the considered QPSK and 16QAM modulations are, respectively, given by

$$
\left.\mathrm{BER}_{\mathrm{NL}, k}\right|_{\mathrm{QPSK}}=Q\left(\frac{1}{2} \sqrt{\frac{\left(2-R_{E}-\sigma_{c}^{2} / \sigma_{s}^{2}\right)^{2}}{\left(\mathrm{SNR}_{\mathrm{AWGN}, k}\right)^{-1}+\frac{K}{L} \varepsilon_{k}\left(\sigma_{c}^{2} / \sigma_{s}^{2}-\frac{1}{4}\left(R_{E}+\sigma_{c}^{2} / \sigma_{s}^{2}\right)^{2}\right)}}\right)
$$

and

$$
\left.\mathrm{BER}_{\mathrm{NL}, k}\right|_{\mathrm{QAM}}=\frac{3}{4} Q\left(\frac{1}{2} \sqrt{\frac{\frac{1}{5}\left(2-R_{E}-\sigma_{c}^{2} / \sigma_{s}^{2}\right)^{2}}{\left(\mathrm{SNR}_{\mathrm{AWGN}, k}\right)^{-1}+\frac{K}{L} \varepsilon_{k}\left(\sigma_{c}^{2} / \sigma_{s}^{2}-\frac{1}{4}\left(R_{E}+\sigma_{c}^{2} / \sigma_{s}^{2}\right)^{2}\right)}}\right)
$$

The importance of such a BER expression is that it is formulated based on the signal characteristics in relation to the PD-HPA characteristics. Accordingly, knowing such signal characteristics provides an efficient tool that gives potential insight into the effects of nonlinear distortion before the signal even hits the amplifier. Moreover, these characteristics can be adopted as key features in several techniques in the literature; such as precoding, SLM, or PTS, where the input signal to the HPA is modified to have the minimum of these characteristics as will be demonstrated later in Chapters 5 and 6 . Thus, an improved performance of the system is expected.

The "apparent" SNR per bit measured at the $k$ th receiver input is the ratio of the power of the transmitted signal including nonlinearity, $\sigma_{d, k}^{2}=\left|\alpha_{0}\right|^{2} \sigma_{s, k}^{2}+\sigma_{n, k}^{2}$, to the noise power $\sigma_{W}^{2}$, that is 


$$
\begin{aligned}
\left.\mathrm{SNR}_{\mathrm{NL}, k}\right|_{\mathrm{app}} & =\frac{1}{\log _{2} M} \frac{\sigma_{d, k}^{2}}{\sigma_{W}^{2}}=\frac{1}{\log _{2} M} \frac{\sigma_{s, k}^{2}\left(1-R_{E}\right)}{\frac{N_{0}}{2}} \\
& =\frac{2 E_{b}\left(1-R_{E}\right)}{N_{0}}=\frac{\left.2 E_{b}\right|_{\mathrm{app}}}{N_{0}}
\end{aligned}
$$

where

$$
\left.E_{b}\right|_{\text {app }}=E_{b}\left(1-R_{E}\right)
$$

and the relation between $\mathrm{SNR}_{k} l_{\text {app }}$ and $\mathrm{SNR}_{\mathrm{AWGN}, k}$ is given by

$$
\left.\mathrm{SNR}_{\mathrm{NL}, k}\right|_{\mathrm{app}}=\mathrm{SNR}_{\mathrm{AWGN}, k}\left(1-R_{E}\right)
$$

The derived BER expressions in (3.34) and (3.35) are computed and compared against the BER measured for simulated 64-user CDMA signals with Walsh codes of length $L=64$. Two modulation formats are considered in the validation of the derived BER performance: QPSK and 16QAM. The measured BER presented in this section represent the average BER of 100 frames. Each frame consists in 10,000 random data symbols. Fig. 3.4 and Fig. 3.5 show the BER curves versus $\left.E_{b}\right|_{\text {app }} / N_{0}$, where it is evident that good agreement exists between the analytic curves and the measured points for all IBO levels. 


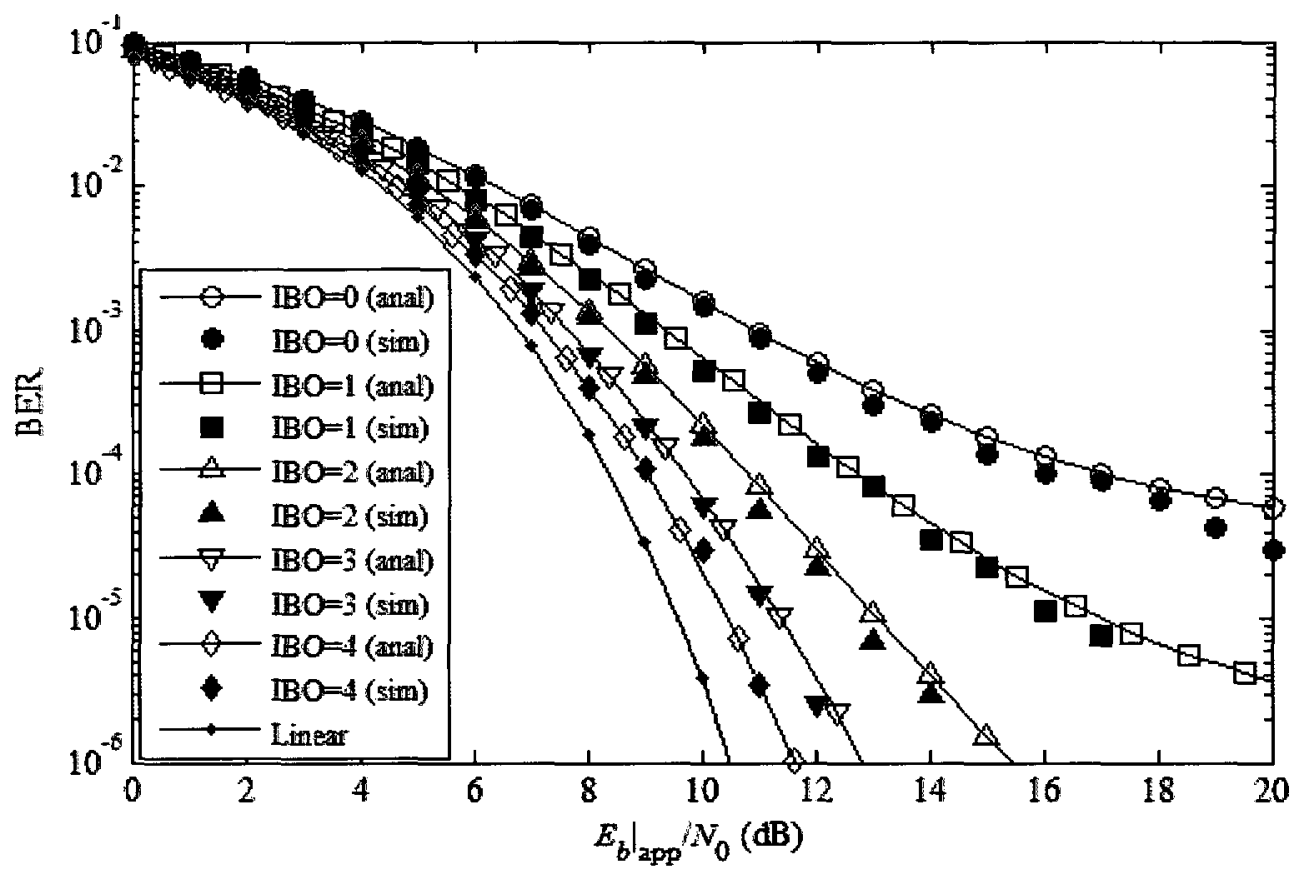

Fig. 3.4 Comparison of the analytic BER performance against measured BER from simulations for QPSK 64-user CDMA signal at different IBOs.

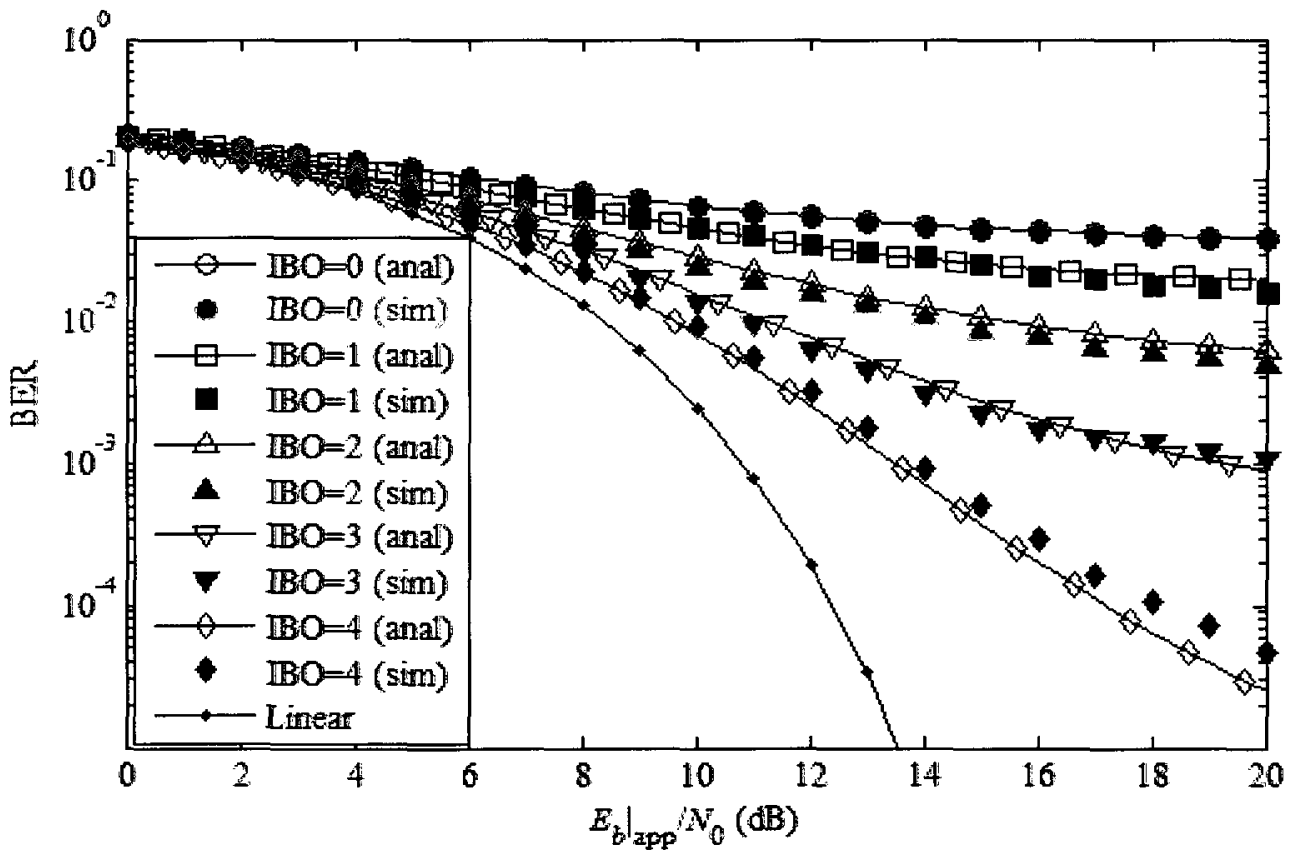

Fig. 3.5 Comparison of the analytic BER performance against measured BER from simulations for 16QAM 64-user CDMA signal at different IBOs. 
It is also worth mentioning the impact of the ratio of the number of users to the spreading factor $(K / L)$ on the BER performance. Fig. 3.6 shows the BER performance for a 64-user CDMA signal modulated using QPSK modulation at IBO of $2 \mathrm{~dB}$ and spreading factors of $L=64,128$, and 256 , where it is clear that the lower this ratio the better the performance. Such an interpretation is also consistent with the findings reported in [50].

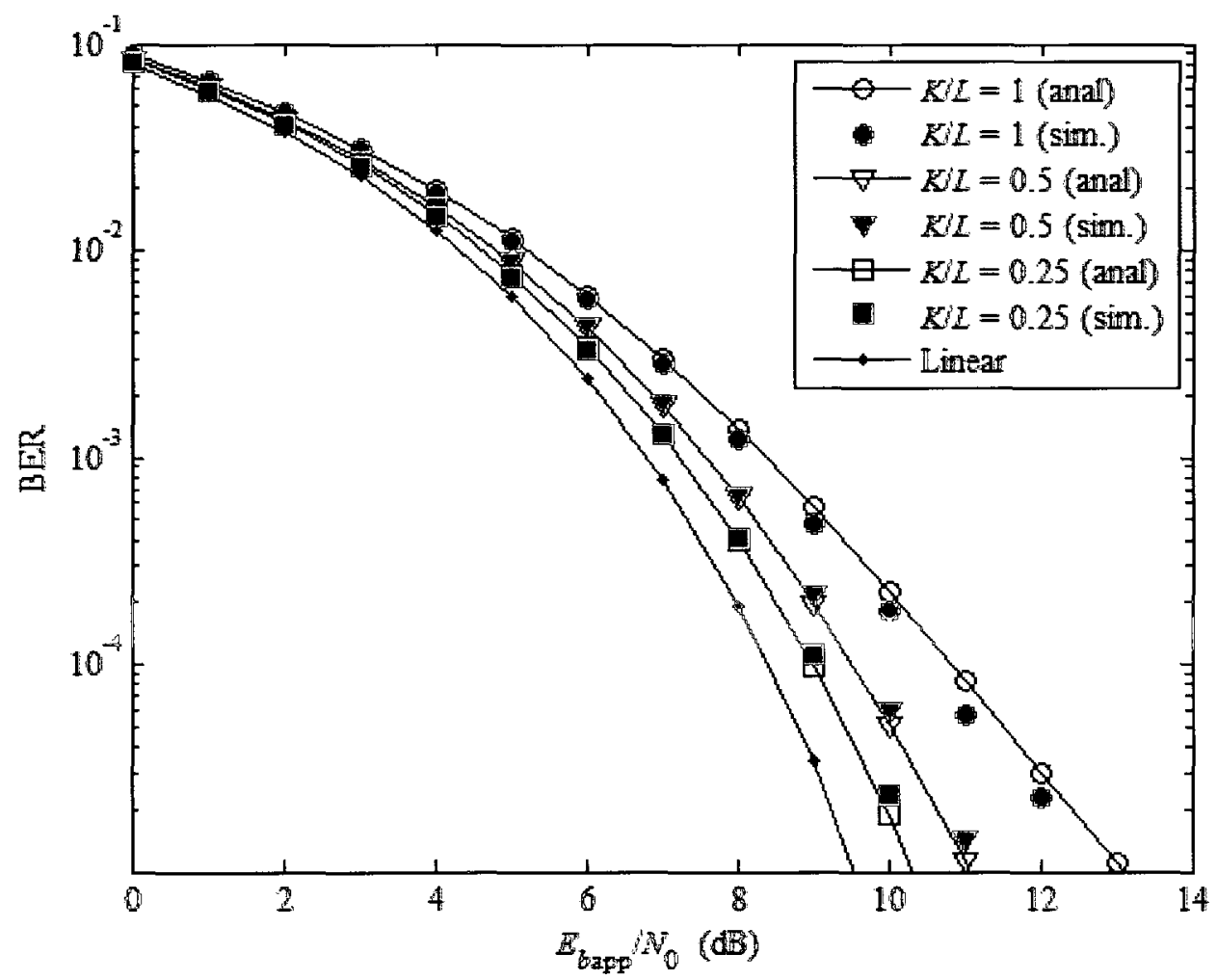

Fig. 3.6 Effect of the ratio $K / L$ on the BER performance of a 64-CDMA signal at IBO $\gamma=2 \mathrm{~dB}$. 


\subsection{BER performance analysis for OFDM signals}

Since it does not impact the goal of the analysis approach in this dissertation, the effect of the guard interval required to alleviate the ISI is not considered for brevity. Consider the OFDM signal in (2.41), where the complex data symbols $A_{k}$ are assumed equiprobable, i.i.d. with zero mean and variance of $E\left\{\left|A_{k}\right|^{2}\right\}$. Also, symbols $A_{k} \in$ $\left\{\chi_{i}\right\}_{i=1, \ldots, M}$, where $\chi_{i} \in\{ \pm 1 \pm j\} / \sqrt{2}$ assuming QPSK modulation. Therefore, the OFDM signal $s(t)$ can be expressed as

$$
\begin{aligned}
s(t) & =\frac{\sqrt{E_{s}}}{N} \sum_{k=1}^{N} A_{k} e^{j \frac{2 \pi k t}{T}}, \quad 0 \leq t \leq T \\
& =x(t)+j y(t)=r(t) e^{j \theta(t)}
\end{aligned}
$$

where $r(t)=|s(t)|=\sqrt{x^{2}(t)+y^{2}(t)}$ and $\theta(t)=\angle s(t)$ are the envelope and phase of $s(t)$, respectively.

In order to proceed in the theoretical analysis of OFDM signals, some assumptions are necessary. It is assumed that the baseband OFDM signal of (3.39) converges to a complex Gaussian random process for large number of subcarrier $N$. Such an assumption can be justified as follows. At any given time instant, the two signal components $x(t)$ and $y(t)$ are given by the sum of i.i.d. zero mean random variables, therefore, $x(t)$ and $y(t)$ can be regarded as two uncorrelated zero-mean Gaussian 
random processes with equal variances, $\sigma_{x}^{2}=\sigma_{y}^{2}$ because of the Central Limit Theorem $[21,44]$. Consequently, $s(t)$ can be regarded as a zero-mean complex Gaussian process with variance

$$
\sigma_{s}^{2}=2 \sigma_{x}^{2}=\frac{E_{s}}{N^{2}} \sum_{k=1}^{N} E\left\{\left|A_{k}\right|^{2}\right\}=\frac{E_{s}}{N},
$$

and its envelope $r(t)$ has a quasi-Rayleigh distribution, whose pdf is given in (3.6).

In presence of the PD-HPA, whose characteristics are given in (3.1), its output can be expressed as

$$
s_{d}(t)=s(t)-s_{c}(t)=r_{d}(t) e^{j \theta(t)},
$$

where $s_{c}(t)=r_{c}(t) e^{j \theta(t)}$ is the signal portion that is clipped from the input signal $s(t)$ by the PD-HPA and $E\left\{s_{c}(t)\right\}=0$.

It is clear that the OFDM signal has a similar statistical characterization to that of the CDMA signal, provided that the number of users and subcarriers are large. In turn, we can apply the same analysis approach pursued for the CDMA signals to OFDM signals. Since the OFDM signal $s(t)$ has been modeled as a zero-mean Gaussian process, we can invoke the complex extension of the Bussgang Theorem in order to express the HPA output as the sum of two uncorrelated components: a scaled linear component and a nonlinear component, $n(t),[35,50,51,52]$, that is 


$$
s_{d}(t)=\alpha_{0} s(t)+n(t)
$$

where $E\{n(t)\}=0, E\left\{s(t) n^{*}(t+\tau)\right\}=0, \forall t, \tau$, and $\alpha_{0}$ is the linear gain given by (3.10). Consequently, the variance of the distorted output signal $s_{d}(t)$ is given by

$$
\sigma_{d}^{2}=\left|\alpha_{0}\right|^{2} \sigma_{s}^{2}+\sigma_{n}^{2}
$$

where $\sigma_{n}^{2}$ is the variance of the nonlinear component $n(t)$. The variance of the distorted output signal $\sigma_{d}^{2}$ for the zero-mean complex Gaussian process $s(t)$ can be expressed as

$$
\sigma_{d}^{2}=\sigma_{s}^{2}\left(1-\exp \left(-\frac{\zeta^{2}}{2 \sigma_{x}^{2}}\right)\right)
$$

At the receiver, the signal is perturbed by a complex zero-mean AWGN $w(t)$ with power spectral density of $N_{0} / 2$, as expressed by

$$
u(t)=s_{d}(t)+w(t)=\alpha_{0} s(t)+n(t)+w(t)
$$

The received signal $u(t)$ is filtered by the receiver filter $g(t)$, which is assumed to be matched to the transmitter filter $h(t)$, i.e., $G(f)=H^{*}(f) / T$. A practical example to ensure the time invariability of $\alpha_{0}$ is the use of a raised-cosine functions with small rolloff factor $\alpha$, i.e., $H(f) G(f)=C G(f)$. The filtered signal $v(t)$ is sampled at instants $n T$, giving

$$
v_{n}=\alpha_{0} s_{n}+n_{n}+w_{n} .
$$


The sampled sequence after the FFT block is given by

$$
V_{k}=\alpha_{0} \sqrt{E_{s}} A_{k}+N_{k}+W_{k}
$$

where

$$
W_{k}=\sum_{n=1}^{N} w_{n} e^{-\frac{j 2 \pi n k}{N}}
$$

is the additive Gaussian noise component with variance $\sigma_{W}^{2}=N \sigma_{W}^{2}=N N_{0} / 2$ and

$$
N_{k}=\sum_{n=1}^{N} n_{n} e^{-\frac{j 2 \pi n k}{N}}
$$

is the nonlinear component due to the nonlinear device, which represents the sum of $N$ uncorrelated random variables. Therefore, for large $N, N_{k}$ can be assumed to be complex Gaussian because of the central limit theorem and its variance is given by $\sigma_{N}^{2}=N \sigma_{n}^{2}$. Consequently, in presence of AWGN only (without nonlinearity), the SNR per bit at the decision device input is given by

$$
\mathrm{SNR}=\mathrm{SNR}_{\mathrm{AWGN}}=\frac{E_{s} / \log _{2} M}{\sigma_{W}^{2}}=\frac{2 E_{b}}{N N_{0}} .
$$

The SNR per bit in presence of both nonlinear distortion and AWGN at the input of the decision device can be evaluated as (see (3.43)) 


$$
\begin{aligned}
\mathrm{SNR}=\mathrm{SNR}_{\mathrm{NL}} & =\frac{\left|\alpha_{0}\right|^{2} E_{s} / \log _{2} M}{\sigma_{W}^{2}+\sigma_{N}^{2} / \log _{2} M}=\frac{\left|\alpha_{0}\right|^{2} E_{b}}{N \frac{N_{0}}{2}+N \sigma_{n}^{2} / \log _{2} M} \\
& =\frac{\left|\alpha_{0}\right|^{2} E_{b}}{N \frac{N_{0}}{2}+\frac{N}{\log _{2} M}\left(\sigma_{d}^{2}-\left|\alpha_{0}\right|^{2} \sigma_{s}^{2}\right)} .
\end{aligned}
$$

Substituting (3.26) and (3.27) in (3.51), we obtain

$$
\begin{aligned}
\mathrm{SNR}_{\mathrm{NL}} & =\frac{\left(1-R_{E} / 2-\sigma_{c}^{2} / 2 \sigma_{s}^{2}\right)^{2} E_{b}}{N \frac{N_{0}}{2}+\frac{N}{\log _{2} M} \sigma_{s}^{2}\left(1-R_{E}-\left(1-R_{E} / 2-\sigma_{c}^{2} / 2 \sigma_{s}^{2}\right)^{2}\right)} \\
& =\frac{1}{4} \frac{\left(2-R_{E}-\sigma_{c}^{2} / \sigma_{s}^{2}\right)^{2} E_{b}}{N \frac{N_{0}}{2}+E_{b}\left(\sigma_{c}^{2} / \sigma_{s}^{2}-\frac{1}{4}\left(R_{E}+\sigma_{c}^{2} / \sigma_{s}^{2}\right)^{2}\right)} \\
& =\frac{1}{4} \frac{\left(2-R_{E}-\sigma_{c}^{2} / \sigma_{s}^{2}\right)^{2}}{\left(\mathrm{SNR}_{\mathrm{AWGN}}\right)^{-1}+\sigma_{c}^{2} / \sigma_{s}^{2}-\frac{1}{4}\left(R_{E}+\sigma_{c}^{2} / \sigma_{s}^{2}\right)^{2}} .
\end{aligned}
$$

It is concluded from (3.52) that, for OFDM signals, the effect of the nonlinear distortion due to the amplifier can be characterized by the signal characteristics $R_{E}$ and $\sigma_{c}^{2}$. Such a finding is expected since OFDM and CDMA have the same statistical characterization as both can be modeled as complex Gaussian processes, provided large number of subcarriers and users, respectively. In turn, the amplifier nonlinearity affects both signals in almost the same manner. 


\subsubsection{Analysis validations and interpretations}

In order to verify the analytic approach pursued to characterize the effect of the nonlinear distortion on OFDM signals, the BER for the QPSK modulation is given by

$$
\left.\mathrm{BER}_{\mathrm{NL}}\right|_{\mathrm{QPSK}}=Q\left(\frac{1}{2} \sqrt{\frac{\left(2-R_{E}-\sigma_{c}^{2} / \sigma_{s}^{2}\right)^{2}}{\left(\mathrm{SNR}_{\mathrm{AWGN}, k}\right)^{-1}+\sigma_{c}^{2} / \sigma_{s}^{2}-\frac{1}{4}\left(R_{E}+\sigma_{c}^{2} / \sigma_{s}^{2}\right)^{2}}}\right)
$$

The analytic BER expressions in (3.53) is computed and compared against the BER measured for a simulated QPSK modulated OFDM signals with $N=256$ subcarriers. The measured BER represent the average BER of 1,000,000 OFDM blocks. Each block consists of 256 random data symbols. Results are obtained at three IBO levels $\gamma=1 \mathrm{~dB}, 2 \mathrm{~dB}$, and $3 \mathrm{~dB}$.

Fig. 3.7 shows the BER curves versus $\left.E_{b}\right|_{\text {app }} / N_{0}$, where it is evident that good agreement exists between the analytic curves and the measured points for all IBO levels. Also, the curves plotted based on the derived BER performance at the IBO levels of interest seem consistent with the measurements in [43, Figure 3.4 (b)] as shown in Fig. 3.8 , with keeping in mind that $E_{b} / N_{0}(\mathrm{~dB})=\mathrm{SNR}(\mathrm{dB})-10 \log _{10}\left(\log _{2} M\right)$. 


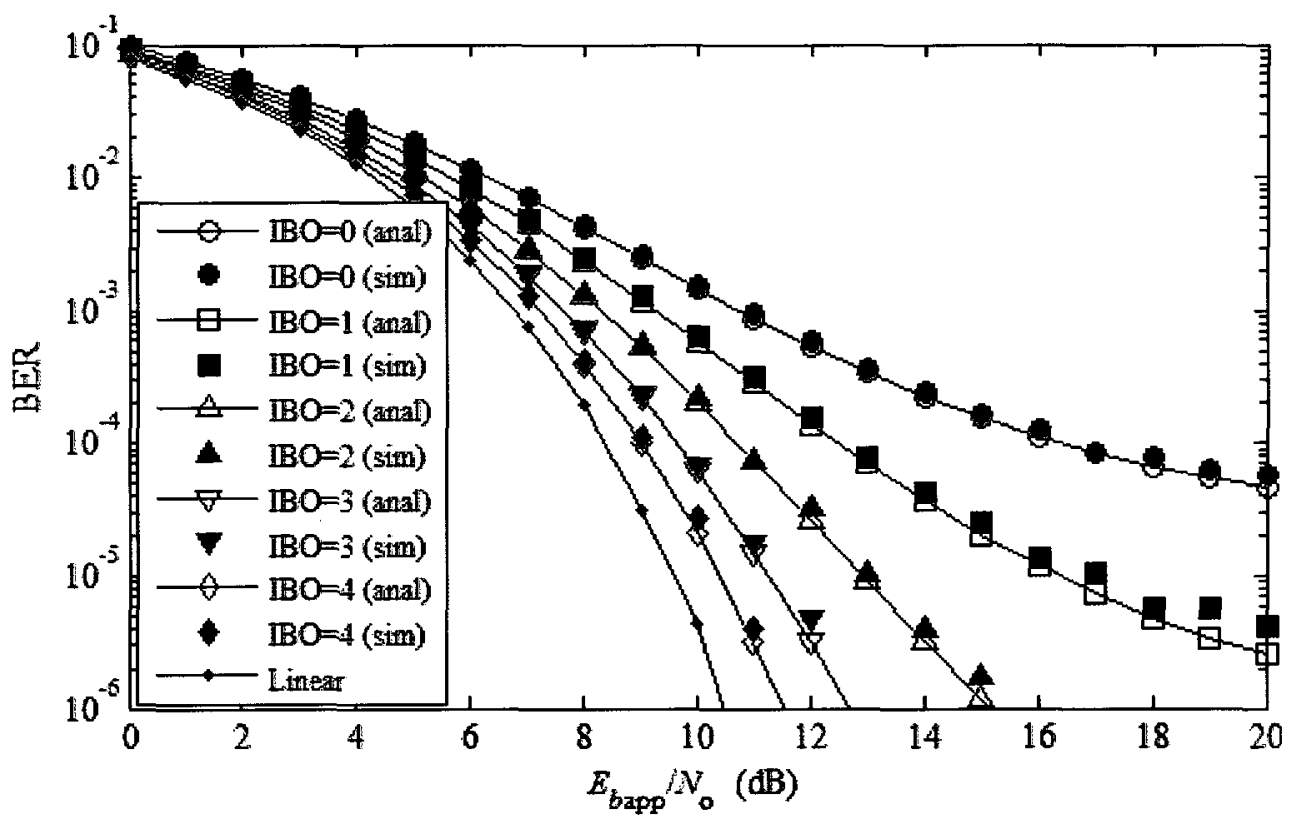

Fig. 3.7 Comparison of the analytic BER performance against measured BER from simulations for QPSK 256-OFDM signal at different IBOs.

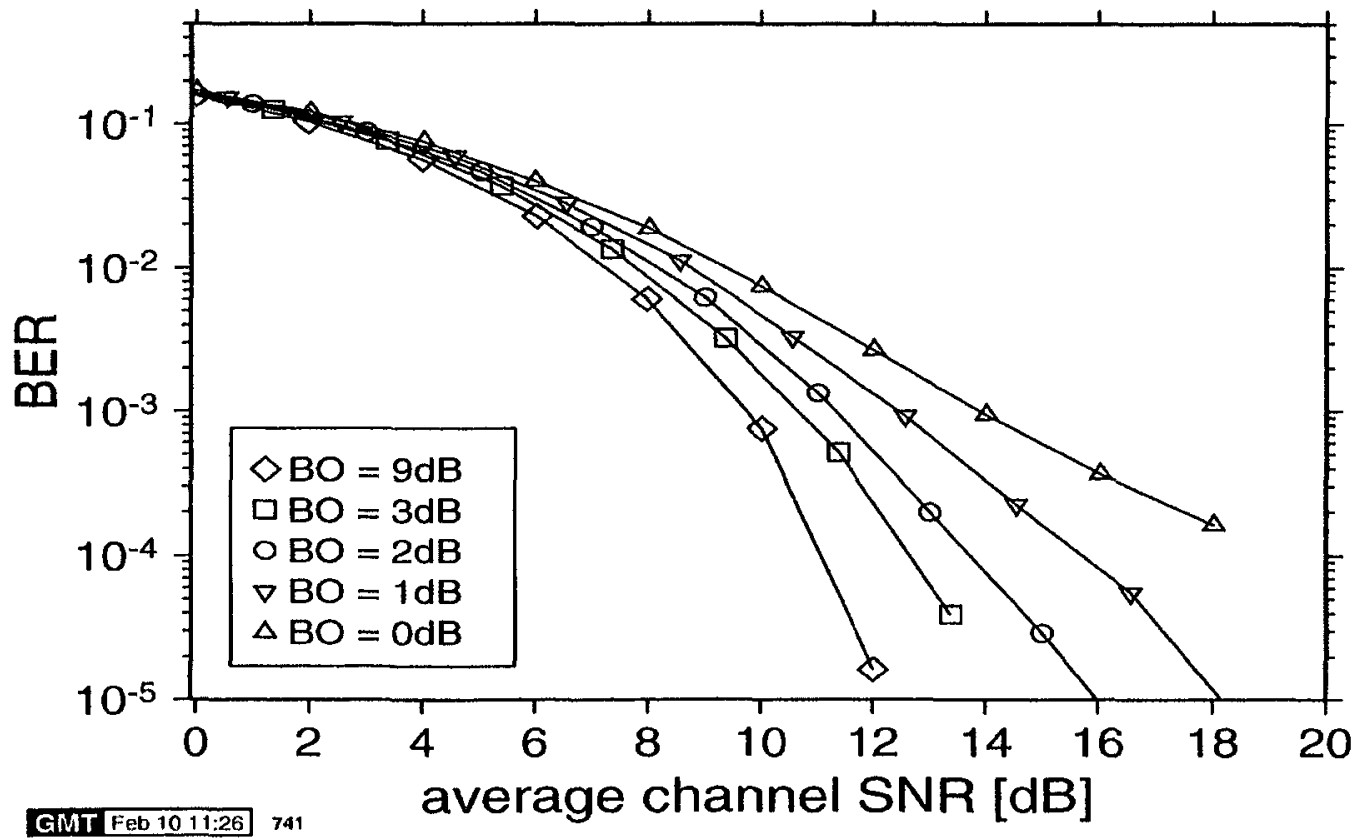

Fig. 3.8 BER versus SNR of QPSK 256-OFDM signal at different IBOs [43]. 


\subsection{SNR relative efficiency}

In the previous analysis, we focused on the nonlinear distortion effects on the BER, where the BER represents the main desirable performance merit in communication systems [36]. In this section, we additionally investigate another performance merit derived from the BER, namely SNR relative efficiency, that turns out to be useful in the analysis, design, and further understanding of the effects of the amplifier nonlinearities.

The efficiency was originally defined for multiuser communication systems as ratio of effective to actual SNR of each user-the effective SNR being that required to achieve the same BER in the absence of interfering users [36, 37]. In the context of this dissertation, the relative efficiency $\eta$ is defined as the ratio of SNRs with and without the presence of nonlinear distortion. Accordingly, the SNR in (3.28) can be rewritten as

$$
\mathrm{SNR}_{\mathrm{NL}, k}=\mathrm{SNR}_{\mathrm{AWGN}, k} \cdot \eta(\gamma)
$$

where

$$
\begin{aligned}
\eta(\gamma) & =\frac{\left|\alpha_{0}\right|^{2}}{1+\frac{\sigma_{N}^{2}}{\sigma_{W}^{2} \log _{2} M}} \\
& =\frac{\left(1-e^{-\gamma}+\frac{1}{2} \sqrt{\pi \gamma} \operatorname{erfc}(\sqrt{\gamma})\right)^{2}}{1+\frac{2 K E_{b}}{N_{0} L}\left(e^{-\gamma}-\sqrt{\pi \gamma} \operatorname{erfc}(\sqrt{\gamma})-\left(e^{-\gamma}-\frac{1}{2} \sqrt{\pi \gamma} \operatorname{erfc}(\sqrt{\gamma})\right)^{2}\right)} \leq 1
\end{aligned}
$$


is the SNR relative efficiency, which represents the SNR attenuation factor (performance loss) due to the amplifier nonlinear distortion. A similar relation can be developed for OFDM signals using (3.52) as

$$
\mathrm{SNR}_{\mathrm{NL}}=\mathrm{SNR}_{\mathrm{AWGN}} \cdot \eta(\gamma),
$$

where

$$
\begin{aligned}
\eta(\gamma) & =\frac{\left|\alpha_{0}\right|^{2}}{1+\frac{\sigma_{N}^{2}}{\sigma_{W}^{2} \log _{2} M}}=\frac{\left|\alpha_{0}\right|^{2}}{1+\frac{\sigma_{n}^{2}}{\sigma_{w}^{2} \log _{2} M}} \\
& =\frac{\left(1-e^{-\gamma}-\frac{1}{2} \sqrt{\pi \gamma} \operatorname{erfc}(\sqrt{\gamma})\right)^{2}}{1+\frac{2 E_{b}}{N N_{0}}\left(e^{-\gamma}-\sqrt{\pi \gamma} \operatorname{erfc}(\sqrt{\gamma})-\left(e^{-\gamma}-\frac{1}{2} \sqrt{\pi \gamma} \operatorname{erfc}(\sqrt{\gamma})\right)^{2}\right)} \leq 1
\end{aligned}
$$

The relation of $\mathrm{SNR}_{\mathrm{NL}}$ versus IBO $\gamma$ at different values of $\mathrm{SNR}_{\mathrm{AWGN}}$, in (3.56), is shown in Fig. 3.9 for an example 256-OFDM signal, at $\mathrm{SNR}_{\mathrm{AWGN}}=4,6, \ldots, 30 \mathrm{~dB}$, where it is clear that as the IBO increases, the relative efficiency increases approaching unity and the difference between $\mathrm{SNR}_{\mathrm{NL}}$ and $\mathrm{SNR}_{\mathrm{AWGN}}$ vanishes. Also, at a fixed IBO level, it can be observed that as the $\mathrm{SNR}_{\mathrm{AWGN}}$ increases, the relative efficiency deteriorates, since $\sigma_{w}^{2}$ decreases and in turn the nonlinear distortion becomes dominant. 


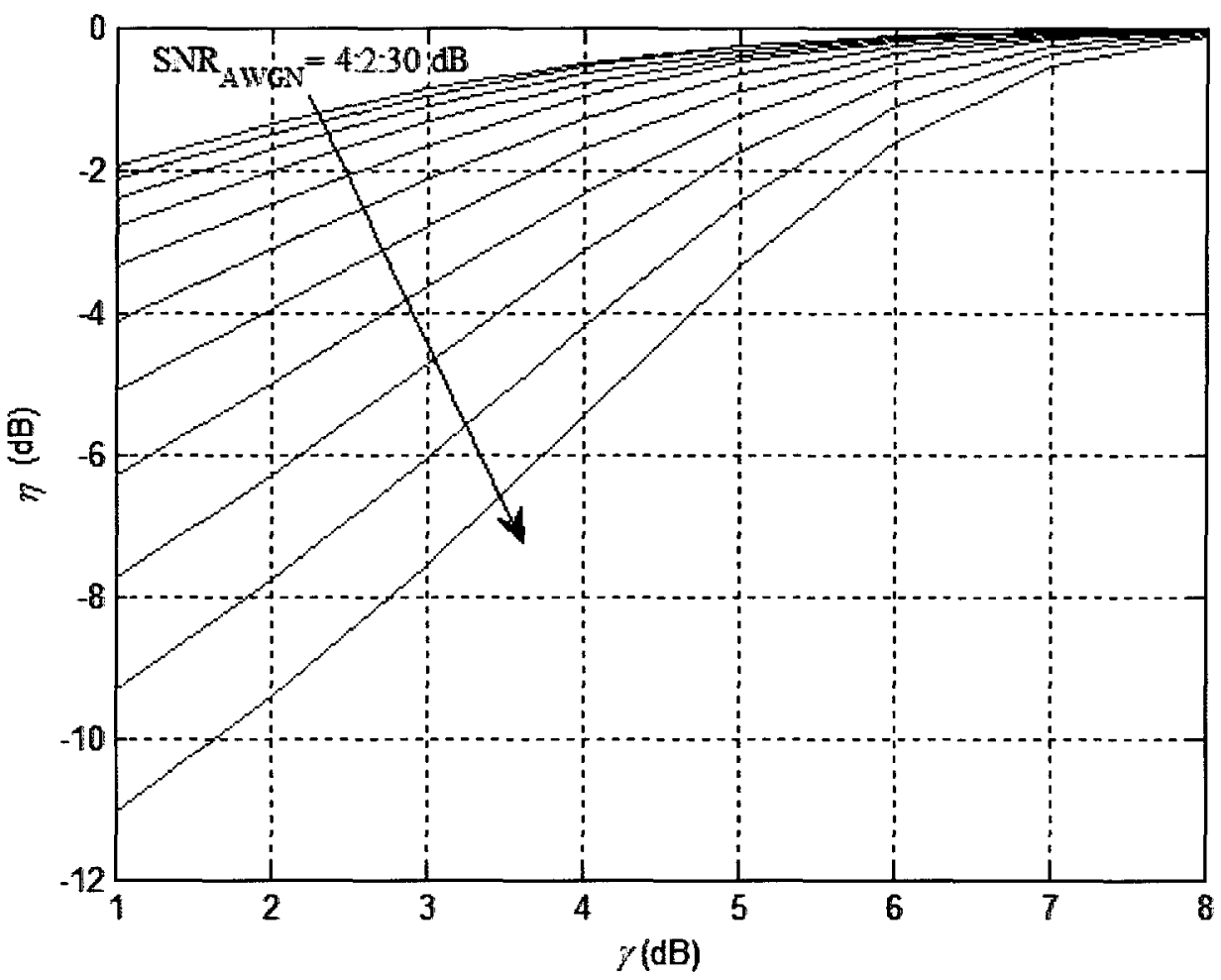

Fig. 3.9 SNR relative efficiency for a 256-OFDM signal at different IBOs.

Finally, based on the reformulation of the relation between $\mathrm{SNR}_{\mathrm{NL}}$ and $\mathrm{SNR}_{\mathrm{AWGN}}$ using the relative efficiency $\eta$ that is function in the IBO level, the IBO required to achieve a certain error rate can be determined through developing a direct relation between the BER in presence of $A W G N$ and nonlinear distortion, $B E R_{N L}$, and the $B E R$ in presence of $\mathrm{AWGN}$ only, $\mathrm{BER}_{\mathrm{AWGN}}=Q\left(\sqrt{\mathrm{SNR}_{\mathrm{AWGN}}}\right)$, assuming QPSK modulation, as follows

$$
\mathrm{BER}_{\mathrm{NL}}^{(\gamma)}=Q\left(\sqrt{\mathrm{SNR}_{\mathrm{NL}}}\right)
$$




$$
=Q\left(\sqrt{Q^{-1}\left(\sqrt{\mathrm{BER}_{\mathrm{AWGN}}}\right) \cdot \eta(\gamma)}\right) .
$$

The relation in (3.58) is shown in Fig. 3.10 for a 256-OFDM signal at different IBO levels. By knowing the BER in the presence of AWGN only, the required IBO level can be determined directly from the curves in Fig. 3.10 as the one that achieves certain required $\mathrm{BER}$ in presence of the nonlinear distortion. Again, it can be observed that as the BER $_{\mathrm{AWGN}}$ decreases, the thermal noise becomes more dominant and, in turn, the difference between $B E R_{N L}$ and $B E R_{A W G N}$ tends to vanish.

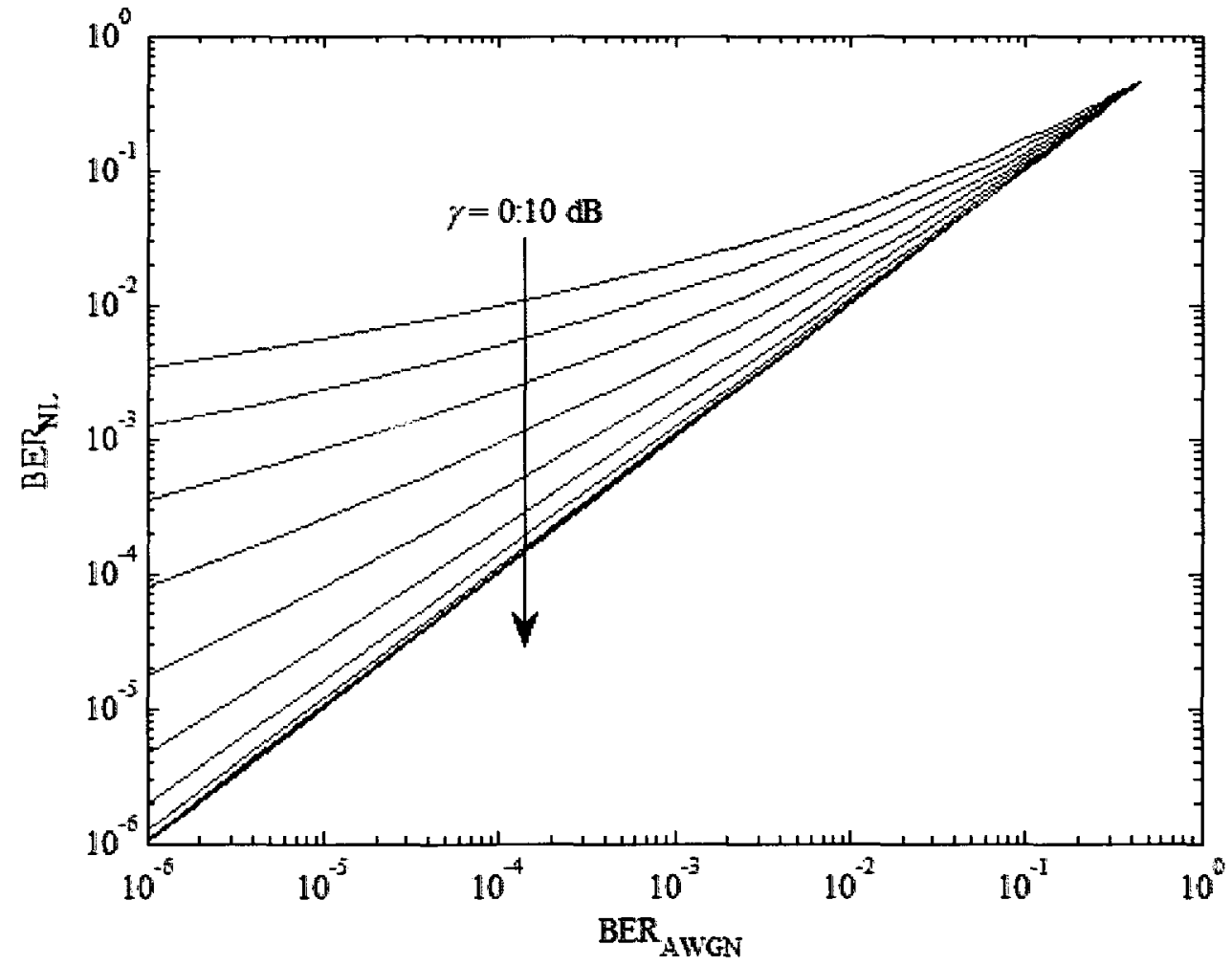

Fig. 3.10 BER $_{\mathrm{NL}}$ versus $\mathrm{BER}_{\mathrm{AWGN}}$ for a QPSK 256-OFDM signal at different IBOs. 


\subsection{Conclusion}

In this chapter, we first analyzed the nonlinear distortion effects caused by a PDHPA on BER degradation for the CDMA and OFDM signals. Based on this analysis, we then established which signal characteristics at the PD-HPA input represent the main contributors to BER degradation. Such characteristics are the threshold exceeding rate of the input signal $R_{E}$ and the variance of the clipped portion of the input signal $\sigma_{c}^{2}$. We developed new BER performance expressions for CDMA and OFDM signals. The importance of such BER expressions is that they are formulated based on the signal characteristics in relation to the PD-HPA characteristics. Accordingly, knowing such signal characteristics provides an efficient tool that gives potential insight into the effects of nonlinear distortion before the signal even hits the amplifier. Moreover, these characteristics can be adopted as key features in several techniques in the literature; such as precoding, SLM, or PTS, where the input signal to the HPA is modified to have the minimum of these characteristics as will be demonstrated later in Chapters 5 and 6. Verification of the derived analytic expressions through simulations shows good agreement and confirms the validity of the assumptions taken during the derivations. Finally, defining the SNR relative efficiency leads to further understanding of the amplifier nonlinear distortion and provides a useful way to determine the IBO level required to achieve a certain BER in presence of both AWGN and amplifier nonlinearity. 


\section{Chapter 4}

\section{ANALYSIS OF NONLINEAR DISTORTION EFFECTS ON SPECTRAL REGROWTH}

$\mathrm{T}$

The RF spectrum is an precious component in wireless communications systems. Service providers have to pay annual fees for the RF spectrum usage according to national and international regulations. These fees represent a substantial part of system cost. For instance, in the US administration budget for 2009, service providers are required to pay as much as $\$ 550$ million per year for the right to hold a spectrum license. Accordingly, efficient spectrum utilization has grabbed great attention of service providers. As we mentioned previously, nonlinear distortion due to HPA creates additional emissions outside the input signal's bandwidth, known as spectral regrowth. This spectral regrowth could have a negative effect on nearby channels and in turn negatively affects the overall system capacity.

In this chapter, we first briefly review the conventional analysis of spectral regrowth due HPA nonlinear distortion. Then, we study the effects of the nonlinear distortion due to PD-HPA on the output PSD. We pursue a piecewise analysis approach to explore which signal characteristics at the PD-HPA input represent the main contributors to spectral regrowth. Finally, we study the special case of synchronous 
CDMA signals with orthogonal spreading codes, where the relation between the Walsh code properties and the resulting spectral regrowth is shown.

\subsection{Conventional analysis of spectral regrowth}

Spectral regrowth has been extensively analyzed in the literature [55-59], where the HPA is modeled by an $n$ th-order polynomial. Using higher-order statistics as a tool for the analysis of spectral regrowth, Zhou [55] developed a closed form expression for the covariance function of the HPA output, which upon taking the Fourier transform gives the PSD, assuming a 3rd-order polynomial HPA model. Then [56] extended the results of [55] to up to 7 th-order nonlinearities and showed how spectral regrowth can be predicted based on measured HPA data.

In [57], Aparin studied the spectral regrowth in the reverse-link CDMA signal. The advantage of [57] is the separation of the components responsible for different forms of nonlinear distortion (in-band distortion and spectral regrowth) in the PSD at the HPA output. For a weakly nonlinear HPA modeled by a 3rd-order polynomial with an input $s(t)$, the distorted output signal $s_{d}(t)$ is given by

$$
s_{d}(t)=a_{1} s(t)+a_{3} s^{3}(t)=a_{1} s(t)+a_{3} s_{3}(t)
$$

where the second-order term is omitted since it contributes to components distant from the signal bandwidth. The autocorrelation function of the output signal $s_{d}(t)$ is 


$$
\begin{aligned}
R_{s_{d} s_{d}}(\tau)= & E\left\{s_{d}(t) s_{d}^{*}(t+\tau)\right\} \\
= & E\left\{\left(a_{1} s(t)+a_{3} s_{3}(t)\right)\left(a_{1}^{*} s^{*}(t+\tau)-a_{3}^{*} s_{3}^{*}(t+\tau)\right)\right\} \\
= & \left|a_{1}\right|^{2} E\left\{s(t) s^{*}(t+\tau)\right\}+a_{1} a_{3}^{*} E\left\{s(t) s_{3}^{*}(t+\tau)\right\} \\
& +a_{1}^{*} a_{3} E\left\{s_{3}(t) s^{*}(t+\tau)\right\}+\left|a_{3}\right|^{2} E\left\{s_{3}(t) s_{3}^{*}(t+\tau)\right\} \\
= & \left|a_{1}\right|^{2} R_{s s}(\tau)+a_{1} a_{3}^{*} R_{s s_{3}}(\tau)+a_{1}^{*} a_{3} R_{s_{3} s}(\tau)+\left|a_{3}\right|^{2} R_{s_{3} s_{3}}(\tau)
\end{aligned}
$$

where $R_{i j}(\tau)$ represents the cross-correlation function of the arbitrary signals $i(t)$ and $j(t)$. The Fourier transform of the autocorrelation function in (4.2) gives the PSD of the output signal, that is

$$
S_{s_{d} s_{d}}(f)=\left|a_{1}\right|^{2} S_{s s}(f)+a_{1} a_{3}^{*} S_{s s_{3}}(f)+a_{1}^{*} a_{3} S_{s_{3} s}(f)+\left|a_{3}\right|^{2} S_{s_{3} s_{3}}(f)
$$

The first summand in (4.3) is the PSD of the linear response of the input signal $s(t)$. The second and third summands are the cross PSDs between the input signal $s(t)$ and the third-order response $s_{3}(t)$, which are responsible for the in-band gain compression or expansion. The last summand is the PSD of the third-order response $s_{3}(t)$ that gives the spectral regrowth. Additionally, [57] gives interesting analytic expressions for such forms of distortion. In [58], Banelli and Cacopardi have introduced a closed form expression for the output spectrum from a nonlinear device, when the OFDM signal is modeled by a complex Gaussian process, as 


$$
S_{s_{d} s_{d}}(f)=\frac{c_{0}}{2 \sigma^{2}} S_{s s}(f)+\sum_{n=1}^{\infty} \frac{c_{n}}{\left(2 \sigma^{2}\right)^{2 n+1}} \cdot\left[S_{s s}(f) \otimes_{1} \cdots \otimes_{2 n+1} S_{s s}(f)\right]
$$

with $c_{n}$ calculated as in [57, eq. (7)], $2 \sigma^{2}$ is the input signal power, and $\otimes$ is the convolution operator. In [58], Gard et al. has also provided general expressions for inband distortion and spectral regrowth, but for a generalized $n$ th-order polynomial model. The advantage of such an expression is the separation of the output power spectrum into logical contributions to both the in-band gain compression/expansion and the out-of-band distortion (spectral regrowth), respectively, as

$$
S_{s_{d} s_{d}}(f)=\left.S_{s_{d} s_{d}}\right|_{\text {Gain }}(f)+\left.S_{s_{d} s_{d}}\right|_{\text {Distorion }}(f)
$$

where

$$
\begin{aligned}
\left.S_{s_{d} s_{d}}\right|_{\text {Gain }}(f)= & \left|a_{1}\right|^{2} S_{s s}(f)+\sum_{n=1}^{\frac{N-1}{2}} \frac{1}{2^{2(n+1)}}\left(\begin{array}{c}
2 n+1 \\
n+1
\end{array}\right) \\
& \cdot\left(a_{1} a_{2 n+1}^{*} S_{s s_{(2 n+1)}}(f)+a_{2 n+1} a_{1}^{*} S_{s_{(2 n+1)}}(f)\right)
\end{aligned}
$$

are the spectral terms correlated to the input signal that represent the gain expansion or compression of the desired signal at the output and 


$$
\left.S_{s_{d} s_{d}}\right|_{\text {Distorion }}(f)=\sum_{n=1}^{\frac{N-1}{2}} \sum_{m=1}^{\frac{N-1}{2}} \frac{a_{2 n+1} a_{2 m+1}^{*}}{2^{2(n+m)}}\left(\begin{array}{c}
2 n+1 \\
n+1
\end{array}\right)\left(\begin{array}{c}
2 m+1 \\
m+1
\end{array}\right) S_{s_{(2 n+1)} s_{(2 m+1)}}(f)
$$

are the cross-correlations of the higher-order components that represent the spectral regrowth.

\subsection{Spectral regrowth analysis}

The approaches in [55-59 and the references therein] primarily look at modeling spectral regrowth due to the HPA nonlinearities, while in this dissertation, the main goal is to explore which signal characteristics at the PD-HPA input represent the main contributors to spectral regrowth. The motivation of such goal is to provide the system designer/operator with an efficient tool to estimate the severity of the spectral regrowth before the signal hits the amplifier chain. Accordingly, one would be able to control such input signal characteristics in order to minimize spectral regrowth.

Consider the input signal $x(t)$ to the nonlinear PD-HPA with clipping threshold $\zeta$, the output from the PD-HPA can be expressed as

$$
x_{d}(t)=x(t)-x_{c}(t)
$$

where $x_{c}(t)$ is the signal portion that is clipped from the input signal $x(t)$ by the PDHPA, which is given by 


$$
x_{c}(t)=\left\{\begin{array}{cc}
x(t)+\zeta & x(t)<-\zeta \\
0 & |x(t)| \leq \zeta \\
x(t)-\zeta & x(t)>\zeta
\end{array}\right.
$$

Examples of the input signal $x(t)$ to the PD-HPA, the corresponding output signal $x_{d}(t)$ of the PD-HPA, and the clipped signal portion $x_{c}(t)$ are shown in Fig. 4.1(a), Fig. 4.1(b), and Fig. 4.1(c), respectively.

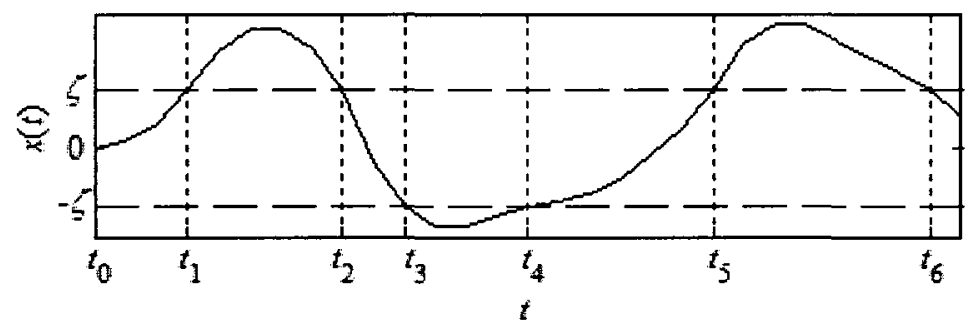

(a)

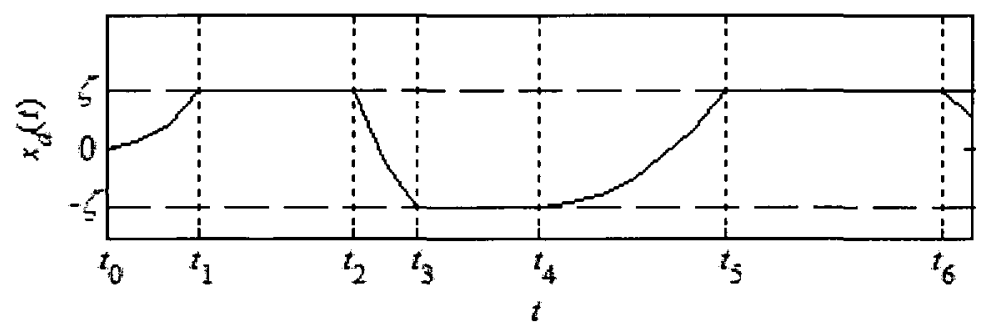

(b)

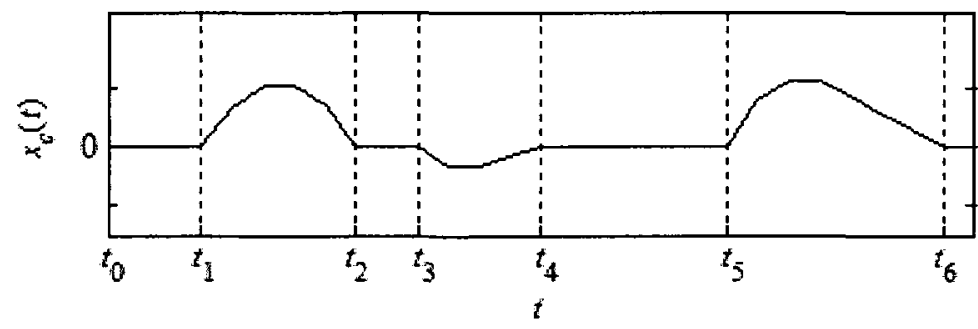

(c)

Fig. 4.1 (a) Input signal $x(t)$ to PD-HPA, (b) corresponding output signal $x_{d}(t)$ from PD-HPA, and (c) clipped signal portion $x_{c}(t)$. 
In investigating the main contributors to spectral regrowth due to the considered nonlinear device, it is convenient to study the PSD at the output of the PD-HPA. The autocorrelation function of the output $x_{d}(t)$ is

$$
\begin{aligned}
R_{x_{d} x_{d}}(\tau) & =E\left\{x_{d}(t) x_{d}(t+\tau)\right\} \\
& =E\left\{\left(x(t)-x_{c}(t)\right)\left(x(t+\tau)-x_{c}(t+\tau)\right)\right\} \\
& =R_{x x}(\tau)-R_{x x_{c}}(\tau)-R_{x_{c} x}(\tau)+R_{x_{c} x_{c}}(\tau)
\end{aligned}
$$

where the first summand in (4.10) is the autocorrelation of the linear response of the input signal $x(t)$ the second and third summands are the cross-correlations between $x(t)$ and $x_{c}(t)$, and the fourth summand is the autocorrelation of the clipped signal portion $x_{c}(t)$. The Fourier transform of the autocorrelation function in (4.10) gives the PSD of the output signal, that is

$$
S_{x_{d} x_{d}}(\omega)=S_{x x}(\omega)-S_{x x_{c}}(\omega)-S_{x_{c} x}(\omega)+S_{x_{c} x_{c}}(\omega),
$$

where $S_{x x}(\omega)$ is the PSD of the input signal to the PD-HPA, $S_{x x_{c}}(\omega)$ and $S_{x_{c} x}(\omega)$ are the cross PSDs of the input signal and the clipped signal portion, and $S_{x_{c} x_{c}}(\omega)$ is the PSD of the clipped signal portion. This relation can be seen in Fig. 4.2 (a) and Fig. 4.2 (b) that show the PSDs in (4.11) for two examples of a 16-user DS-CDMA signal and a 64channel OFDM signal, both filtered by an SRRC filter with roll-off of 0.22 . The PSDs are calculated using the Welch estimation method with $50 \%$ overlap. 

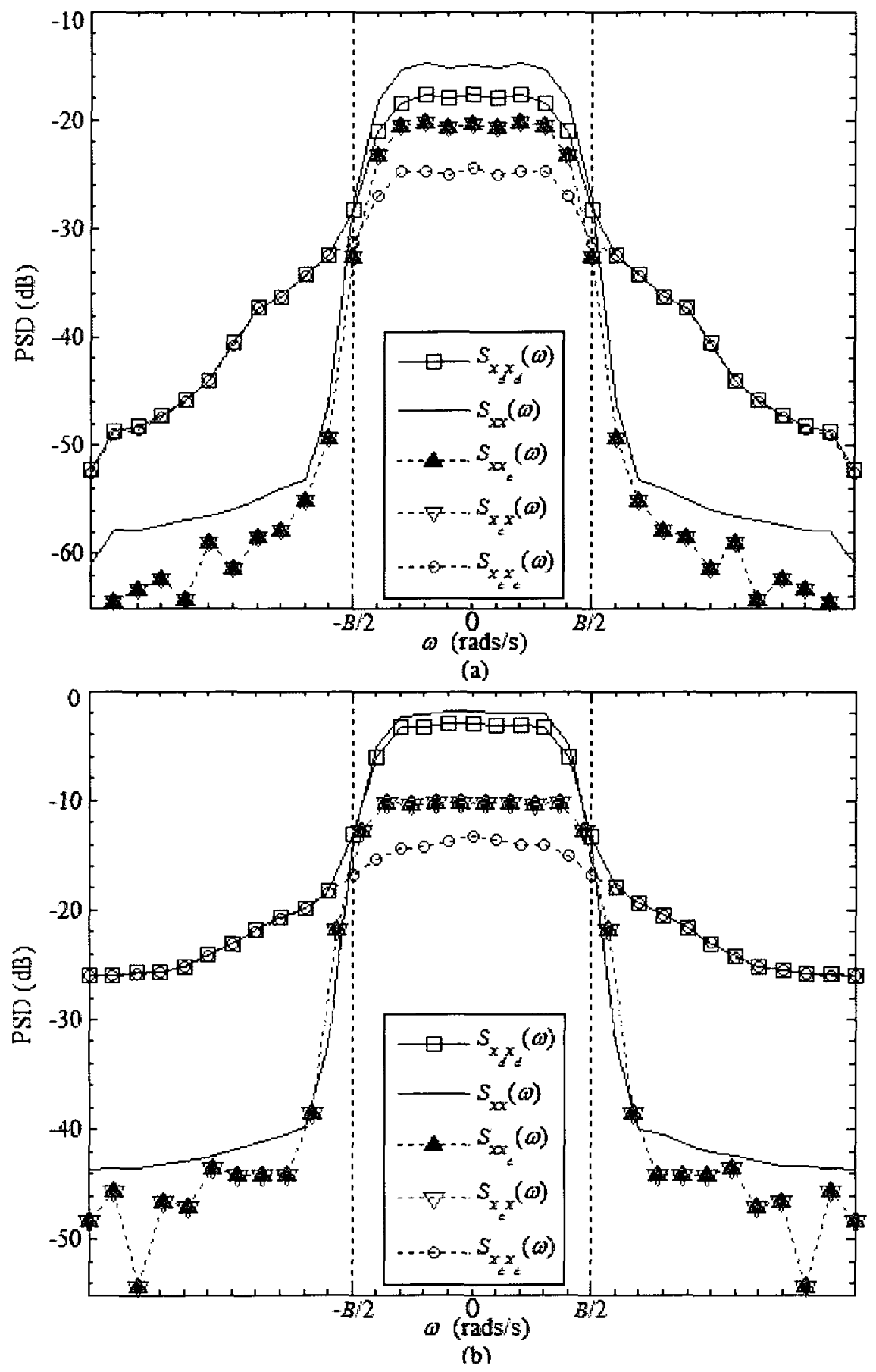

Fig. 4.2 PSDs $S_{x_{d} x_{d}}(\omega), S_{x x}(\omega), S_{x x_{c}}(\omega), S_{x_{c} x}(\omega)$, and $S_{x_{c} x_{c}}(\omega)$ in (4.11) for: (a) 16user CDMA signal and (b) 64-channel OFDM signal. 
It is clear that the cross PSDs $\left(S_{x x_{c}}(\omega), S_{x_{c} x}(\omega)\right)$ between the input signal $x(t)$ and the clipped signal portion $x_{c}(t)$ have very low energies outside the signal bandwidth $(|\omega|>B / 2)$, and most of the energy is concentrated inside the signal bandwidth. This implies that these cross PSDs have a negligible effect on the out-of-band portion of the output spectrum, and mainly affect the in-band gain compression or expansion. On the other hand, the PSD of the clipped signal portion, $S_{x_{c} x_{c}}(\omega)$, has a high energy outside the signal bandwidth compared to the input PSD, resulting in a significant increase in the out-of-band of the output PSD. Spectral regrowth can be defined for the upper channel as the additional out-of-band power at the output of the HPA, i.e.,

$$
P_{\mathrm{SR}}=\int_{B / 2}^{\infty} S_{x_{d} x_{d}}(\omega)-S_{x x}(\omega) d \omega
$$

As shown in Fig 4.2, the PSD of the clipped signal portion almost coincides with the output distorted PSD outside the signal bandwidth, which means that the energy of the clipped signal portion outside the signal bandwidth represents almost all of the out-ofband energy of the output distorted signal, i.e.

$$
S_{x_{d} x_{d}}(\omega) \cong S_{x_{c} x_{c}}(\omega), \quad|\omega|>\frac{B}{2}
$$

Since the PSDs of the baseband bandlimited CDMA and OFDM signals are almost rectangular for large number of users and large number of subcarriers, respectively [21], 
the input PSD can be assumed constant over the entire bandwidth $B$ and zero elsewhere, i.e.,

$$
S_{x x}(\omega)=0, \quad|\omega|>\frac{B}{2}
$$

Substituting (4.13) and (4.14) in (4.12), gives

$$
P_{\mathrm{SR}} \cong \int_{B / 2}^{\infty} S_{x_{c} x_{c}}(\omega) d \omega
$$

Therefore, it can be concluded that spectral regrowth depends primarily on the PSD of the clipped signal portion and, in turn, as the variance of the clipped signal portion $\sigma_{c}^{2}=\int_{-\infty}^{\infty} S_{x_{c} x_{c}}(\omega) d \omega$ increases, spectral regrowth increases.

Since the signal $x_{c}(t)$ and its PSD are responsible for spectral regrowth, we adopt a piecewise analysis approach for such a signal to determine what other signal characteristics at the PD-HPA input, in addition to $\sigma_{c}^{2}$, would contribute to spectral regrowth. In such an approach, we consider $x_{c}(t)$ as a piecewise signal, following the example in Fig. 4.1(c), where each piecewise segment $x_{c, i}(t)$ is realized in the intervals $\left[t_{i-1}, t_{i}\right)$ with $i=1, \ldots, I$ to accommodate $I$ piecewise segments, where $t_{i}>t_{i-1}$ and with $t_{0}$ arbitrarily assigned as $t_{0}=0 \mathrm{sec}$. Therefore, $x_{c}(t)$ can be represented as a piecewise signal, based on its definition in (4.9), as 


$$
x_{c}(t)=\sum_{i=1}^{I} x_{c, i}(t)
$$

where

$$
x_{c, i}(t)=x(t)\left|w_{\text {rect }, i}\left(t ; \tau_{i}\right)\right|-\zeta w_{\text {rect }, i}\left(t ; \tau_{i}\right)
$$

For our purposes, $w_{\text {rect }, i}\left(t ; \tau_{i}\right)$ are represented by rectangular windows as

$$
w_{\text {rect }, i}\left(t ; \tau_{i}\right)=\left\{\begin{aligned}
\operatorname{sgn}\{x(t)\}, & t_{i}-\tau_{i} \leq t<t_{i} \\
0, & \text { otherwise }
\end{aligned}\right.
$$

where

$$
\operatorname{sgn}\{x(t)\}=\frac{x(t)}{|x(t)|}
$$

and $\tau_{i}=t_{i}-t_{i-1}$ is the duration of the $i$ th piecewise segment.

In our scenario, the piecewise signal is restricted to segments that alternate, as in (4.9), between zero corresponding to $x(t)$ for unclipped regions and $x_{c}(t)$ for the clipped regions, as shown in Fig. 4.1(c). Without loss of generality, we assume that even values of $i$ correspond to time intervals with clipping and odd values of $i$ correspond to time 
intervals with no clipping (i.e., $x_{c}(t)=0$ ). The CDMA signal and the OFDM signal can be represented as in [50, eq. (1)] and [44, eq. (1)], respectively, as

$$
x(t)=\sum_{n=0}^{N-1} x_{n} h(t-n T)
$$

where $h(t)$ is the pulse shaping filter. Following this convention, $x_{c}(t)$ can be rewritten as

$$
x_{c}(t)=\sum_{\substack{i=1 \\ i \text { even }}}^{I} \sum_{n=0}^{N-1} x_{n} h(t-n T)\left|w_{r e c t, i}\left(t ; \tau_{i}\right)\right|-\zeta w_{r e c t, i}\left(t ; \tau_{i}\right)
$$

Taking the Fourier transform of (4.21), the power spectrum of the clipped signal portion can be written as

$$
\begin{aligned}
S_{x_{c} x_{c}}(\omega) & =\left|X_{c}(\omega)\right|^{2} \\
& =\left|\sum_{\substack{i=1 \\
i \text { even }}}^{I} \sum_{k=1}^{K} \sqrt{E_{k}} a_{1}^{(k)} \sum_{l=0}^{L-1} c_{l}^{(k)} e^{-j \omega l T_{c}} G_{i}(\omega)-\zeta W_{\text {rect }, i}(\omega)\right|^{2},
\end{aligned}
$$

where 


$$
G_{i}(\omega)=H(\omega) *\left|w_{\text {rect }, i}(\omega)\right|
$$

and where $*$ is the convolution operator.

It should be clear that in the frequency domain, the rectangular windows $w_{\text {rect }, i}\left(t ; \tau_{i}\right), i=1, \ldots, I$, form a set of sinc functions with main lobe widths that are inversely proportional to the durations $\tau_{i}$ 's of the clipped subintervals in the time domain. At each new clipping event $i$, the sinc function $W_{\text {rect }, i}(\omega)$, when convolved with the frequency response of the pulse shaping filter $H(\omega)$ with arbitrary bandwidth $B$ as in (4.23), results in $G_{i}(\omega)$ with bandwidth $B_{i}>B$. Consequently, given the power spectrum of the clipped signal portion in (4.22), it is concluded that as the number of segments $I$ increases, additional windowing with slower fall-off are introduced causing an increase in the out-of-band power of the clipped signal, and so increases the spectral regrowth. In fact, the number of segments $I$ in a fixed time interval determines the number of zerodepartures and zero-arrivals in the clipped signal portion. These zero-departures/arrivals in the clipped signal portion represent the threshold crossings $N_{C}$ in the input signal to the PD-HPA.

Therefore, it is deduced that the signal characteristics at the input of the PD-HPA that mainly contribute to spectral regrowth in relation to the PD-HPA clipping threshold are: 
1) the variance of the signal portion exceeding the PD-HPA clipping threshold $\sigma_{c}^{2}$,

2) the threshold crossing rate $R_{C}=N_{C} / L$, and

3) the time durations of the crossing events $\tau_{i}$ 's.

The above signal characteristics can be developed for both CDMA and OFDM signals as far as both are characterized as band-limited zero-mean Gaussian processes, provided that the number of users in CDMA signals and the number of subcarriers in OFDM signals are large. In the following, we investigate two cases; when the input signal $s(t)$, with variance $\sigma_{s}^{2}$, to the PD-HPA is purely real and is complex. It is convenient to relate the signal characteristics contributing to spectral regrowth to the IBO $\gamma$ instead of the threshold $\zeta$, where $\gamma$ is related to $\zeta$ as $\gamma=\zeta^{2} / \sigma_{s}^{2}$. When the input signal $s(t)=x(t)$ is purely real with variance $\sigma_{x}^{2}$, it has a pdf of

$$
f_{x}(x)=\frac{1}{\sqrt{2 \pi \sigma_{x}^{2}}} \exp \left(-\frac{x^{2}}{2 \sigma_{x}^{2}}\right)
$$

When the input signal to the PD-HPA is given by $s(t)=x(t)+j y(t)$, where $x(t)$ and $y(t)$ are characterized as band-limited zero-mean Gaussian processes with variances $\sigma_{x}^{2}=\sigma_{y}^{2}, s(t)$ is characterized as a band-limited complex zero-mean Gaussian process with variance of $\sigma_{s}^{2}=2 \sigma_{x}^{2}$ and its envelope $r(t)$ can be regarded as a Rayleigh random process with the pdf in (3.6). 
The variance $\sigma_{c}^{2}$ of the clipped signal portion $s_{c}(t)$, where $E\left\{s_{c}(t)\right\}=0$, for both real and complex signals can be, respectively, calculated using (4.9) and (4.24) as

$$
\begin{aligned}
\left.\sigma_{c}^{2}\right|_{\text {real }} & =E\left\{\left|s_{c}(t)\right|^{2}\right\}=E\left\{\left|x_{c}\right|^{2}\right\} \\
& =2 \int_{\zeta}^{\infty}(x-\zeta)^{2} f_{x}(x) d x \\
& =\left(\sigma_{x}^{2}+\zeta^{2}\right) \operatorname{erfc}\left(\sqrt{\frac{\zeta^{2}}{2 \sigma_{x}^{2}}}\right)-\sqrt{\frac{2 \sigma_{x}^{2} \zeta^{2}}{\pi}} \exp \left(-\frac{\zeta^{2}}{2 \sigma_{x}^{2}}\right) \\
& =\sigma_{x}^{2}\left((1+\gamma) \operatorname{erfc}\left(\sqrt{\frac{\gamma}{2}}\right)-\sqrt{\frac{2 \gamma}{\pi}} \exp \left(-\frac{\gamma}{2}\right)\right)
\end{aligned}
$$

and using (3.6) and (3.8)

$$
\begin{aligned}
\left.\sigma_{c}^{2}\right|_{\text {complex }} & =E\left\{\left|s_{c}(t)\right|^{2}\right\}=E\left\{r_{c}^{2}(t)\right\} \\
& =\int_{\zeta}^{\infty}(r-\zeta)^{2} f_{r}(r) d r \\
& =2 \sigma_{x}^{2} \exp \left(-\frac{\zeta^{2}}{2 \sigma_{x}^{2}}\right)-\sqrt{2 \pi \sigma_{x}^{2} \zeta^{2}} \operatorname{erfc}\left(\sqrt{\frac{\zeta^{2}}{2 \sigma_{x}^{2}}}\right) \\
& =2 \sigma_{x}^{2}(\exp (-\gamma)-\sqrt{\pi \gamma} \operatorname{erfc}(\sqrt{\gamma})) .
\end{aligned}
$$


Threshold crossing rate for real Gaussian random process is readily available in the literature $[60,61]$ as

$$
\left.R_{C}\right|_{\text {real }}=\frac{1}{\pi} \sqrt{\frac{\sigma_{\dot{x}}^{2}}{\sigma_{x}^{2}}} \exp \left(-\frac{\zeta^{2}}{2 \sigma_{x}^{2}}\right)
$$

where $\sigma_{\dot{x}}^{2}=E\left\{\dot{x}^{2}(t)\right\}$ and the dot denotes the derivative operator.

Substituting $\sigma_{\dot{x}}^{2}$ from (A.3) into (4.27) (see appendix A for determining the variance of the derivative of a random process with rectangular passband), the threshold crossing rate $\left.R_{C}\right|_{\text {real }}$ can be written as

$$
\begin{aligned}
\left.R_{C}\right|_{\text {real }} & =\frac{B}{\pi \sqrt{12}} \exp \left(-\frac{\zeta^{2}}{2 \sigma_{x}^{2}}\right) \\
& =\frac{1}{\pi \sqrt{12}} B \exp \left(-\frac{\gamma}{2}\right)
\end{aligned}
$$

In the derivation of the threshold crossing rate for the case of the complex Gaussian process, we are not only interested in the probability distribution of the envelope $r(t)$, but also in the joint probability distribution of the envelope $r(t)$ and its first derivative $\dot{r}(t)$. The joint pdf of $r$ and $\dot{r}$ is given by (see appendix B)

$$
f_{r, \dot{r}}(r, \dot{r})=\frac{r}{\sqrt{2 \pi \sigma_{x}^{4} \sigma_{\dot{x}}^{2}}} \exp \left(-\frac{1}{2}\left(\frac{r^{2}}{\sigma_{x}^{2}}+\frac{\dot{r}^{2}}{\sigma_{\dot{x}}^{2}}\right)\right)
$$


For a random process $r(t)$, the expected number of points $\tau_{i}$ in an interval of length $T$ that cross a threshold $\zeta$, is given by [60, ch. 16]

$$
\begin{aligned}
E\left\{n_{\zeta}(T)\right\} & =T f_{r}(\zeta) E\{|\dot{r}(t)| \mid r(t)=\zeta\} \\
& =T f_{r}(\zeta) \int_{-\infty}^{\infty}|\dot{r}| f_{\dot{r} \mid r}(\dot{r} \mid \zeta) d \dot{r} \\
& =T \int_{-\infty}^{\infty}|\dot{r}| f_{r, \dot{r}}(\zeta, \dot{r}) d \dot{r} .
\end{aligned}
$$

Substituting (4.29) into (4.30), and integrating with respect to $\dot{r}$, the threshold crossing rate $R_{C} l_{\text {complex }}$ for the signal envelope can be written as

$$
\left.R_{C}\right|_{\text {complex }}=\frac{E\left\{n_{\zeta}(T)\right\}}{T}=\sqrt{\frac{2 \sigma_{\dot{x}}^{2}}{\pi \sigma_{x}^{4}}} \zeta \exp \left(-\frac{\zeta^{2}}{2 \sigma_{x}^{2}}\right)
$$

Substituting $\sigma_{\dot{x}}^{2}$ from (A.3) into (4.31), the threshold crossing rate $R_{C} l_{\text {complex }}$ can be written as

$$
\begin{aligned}
\left.R_{C}\right|_{\text {complex }} & =\sqrt{\frac{\zeta^{2}}{6 \pi \sigma_{x}^{2}}} B \exp \left(-\frac{\zeta^{2}}{2 \sigma_{x}^{2}}\right) \\
& =\sqrt{\frac{\gamma}{3 \pi}} B \exp (-\gamma) .
\end{aligned}
$$


The time durations of the crossing events or sojourn intervals have been addressed in the literature $[61,63]$. Rice [61] has introduced approximate expressions for the distribution of such intervals for both Gaussian and Rayleigh random processes [61, p. 605, and p. 615]. However, these expressions are only valid for very high threshold levels and small values of $\tau_{i}$ 's. Unfortunately, no exact expressions have been developed yet. In 1958 , Rice [61, p. 582] has commented that "although it is generally accepted that the probability of a longer fade interval decreases exponentially, no convenient method of exact calculation is available as yet". In 2007, Morgan [63] comments, "sadly, that still seems to be the state of affairs even today". Therefore, in our approach, we use the mean threshold crossing duration $\bar{\tau}$, as an indicator to the durations of crossing events. Expressions for the mean threshold crossing duration $\bar{\tau}$ of both the real and complex random processes can be found in [61, 63-66], which are given by

$$
\left.\bar{\tau}\right|_{\text {real }}=\pi \sqrt{\frac{\sigma_{x}^{2}}{\sigma_{\dot{x}}^{2}}} \exp \left(\frac{\zeta^{2}}{2 \sigma_{x}^{2}}\right) \cdot \operatorname{erfc}\left(\sqrt{\frac{\zeta^{2}}{2 \sigma_{x}^{2}}}\right)
$$

and

$$
\left.\bar{\tau}\right|_{\text {complex }}=\frac{1}{\zeta} \sqrt{\frac{\pi \sigma_{x}^{4}}{2 \sigma_{\dot{x}}^{2}}}
$$


Substituting $\sigma_{\dot{x}}^{2}$ from (A.3) into (4.33) and (4.34), the mean threshold crossing duration $\bar{\tau}$ of both the real and complex random processes can be respectively expressed by

$$
\begin{aligned}
\left.\bar{\tau}\right|_{\text {real }} & =\frac{\pi \sqrt{12}}{B} \exp \left(\frac{\zeta^{2}}{2 \sigma_{x}^{2}}\right) \cdot \operatorname{erfc}\left(\sqrt{\frac{\zeta^{2}}{2 \sigma_{x}^{2}}}\right) \\
& =\frac{\pi \sqrt{12}}{B} \exp \left(\frac{\gamma}{2}\right) \cdot \operatorname{erfc}\left(\sqrt{\frac{\gamma}{2}}\right)
\end{aligned}
$$

and

$$
\left.\bar{\tau}\right|_{\text {complex }}=\frac{1}{B} \sqrt{\frac{6 \pi \sigma_{x}^{2}}{\zeta^{2}}}=\frac{1}{B} \sqrt{\frac{3 \pi}{\gamma}} .
$$

\subsection{Analysis validation and interpretations}

In order to assess the validity of the developed analytical expressions for the signal characteristics contributing to spectral regrowth in the cases of real and complex processes, two 64-user CDMA signals are simulated as an example for the signal types of interest in this dissertation. The signals are filtered using an SRRC filter with roll-off of 0.22 and oversampled by 4 . The data symbols for the two signals are $a_{n}^{(k)}= \pm 1$ as representative for real signals and $a_{n}^{(k)}=( \pm 1 \pm j) / \sqrt{2}$ as representative for complex signals. Then, $\left.\sigma_{c}^{2}\right|_{\text {real }},\left.R_{c}\right|_{\text {real }}$, and $\left.\bar{\tau}\right|_{\text {real }}$ are measured for the first simulated signal, 
while $\left.\sigma_{c}^{2}\right|_{\text {complex }},\left.R_{c}\right|_{\text {complex }}$, and $\left.\bar{\tau}\right|_{\text {complex }}$ are measured for the second signal, at different values of IBO $\gamma$. The measured results are compared against the computed results using the analytic expressions in (4.25), (4.28), (4.35), (4.26), (4.32), and (4.36). Fig. 4.3, Fig. 4.4, and Fig. 4.5 show the curves of such signal characteristics, where it is evident that good agreement exists between the analytic curves and the measured points for all IBO levels. This agreement confirms the analytical expressions of the signal characteristics developed for CDMA signals and the validity of the assumptions taken during their derivation.

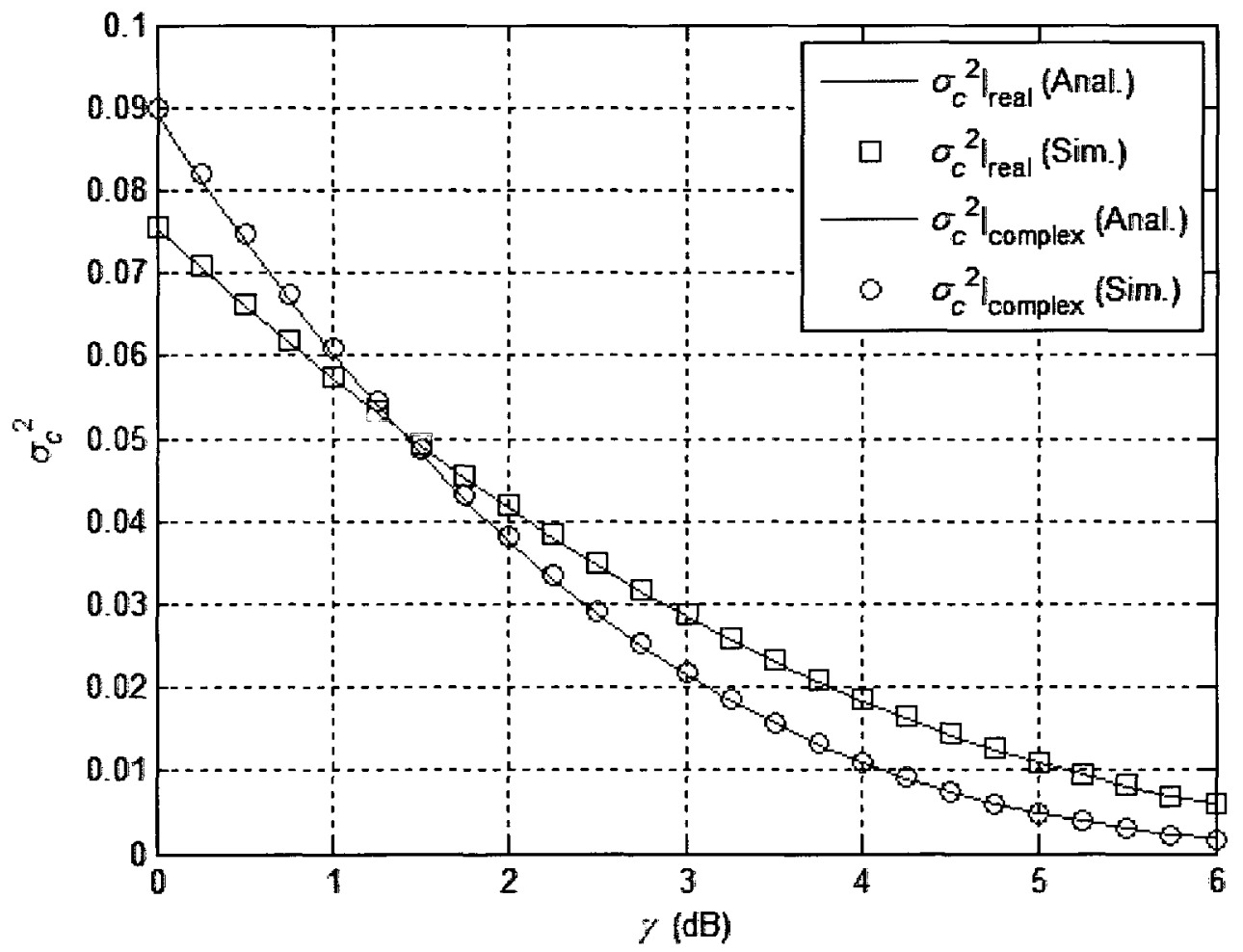

Fig. 4.3 Variances of the clipped signal portion. 


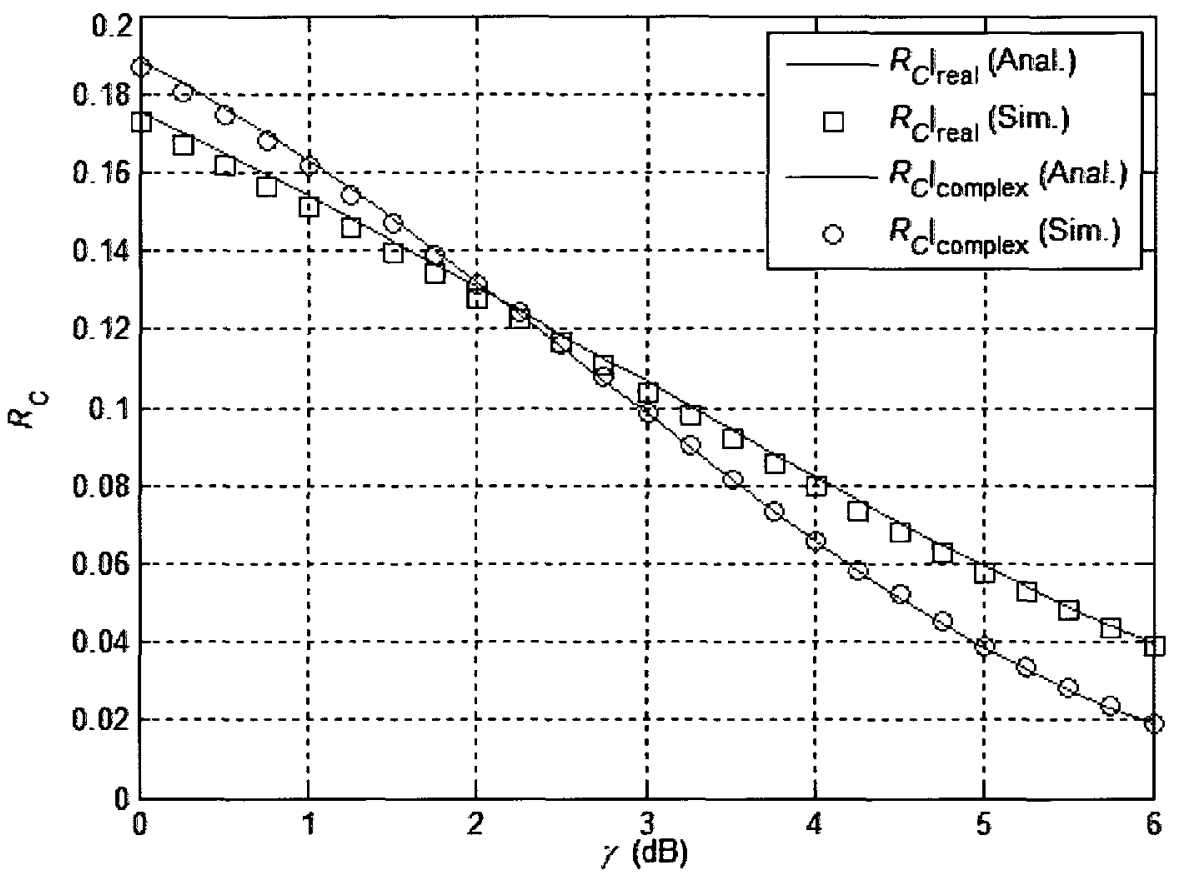

Fig. 4.4 Threshold crossing rates.

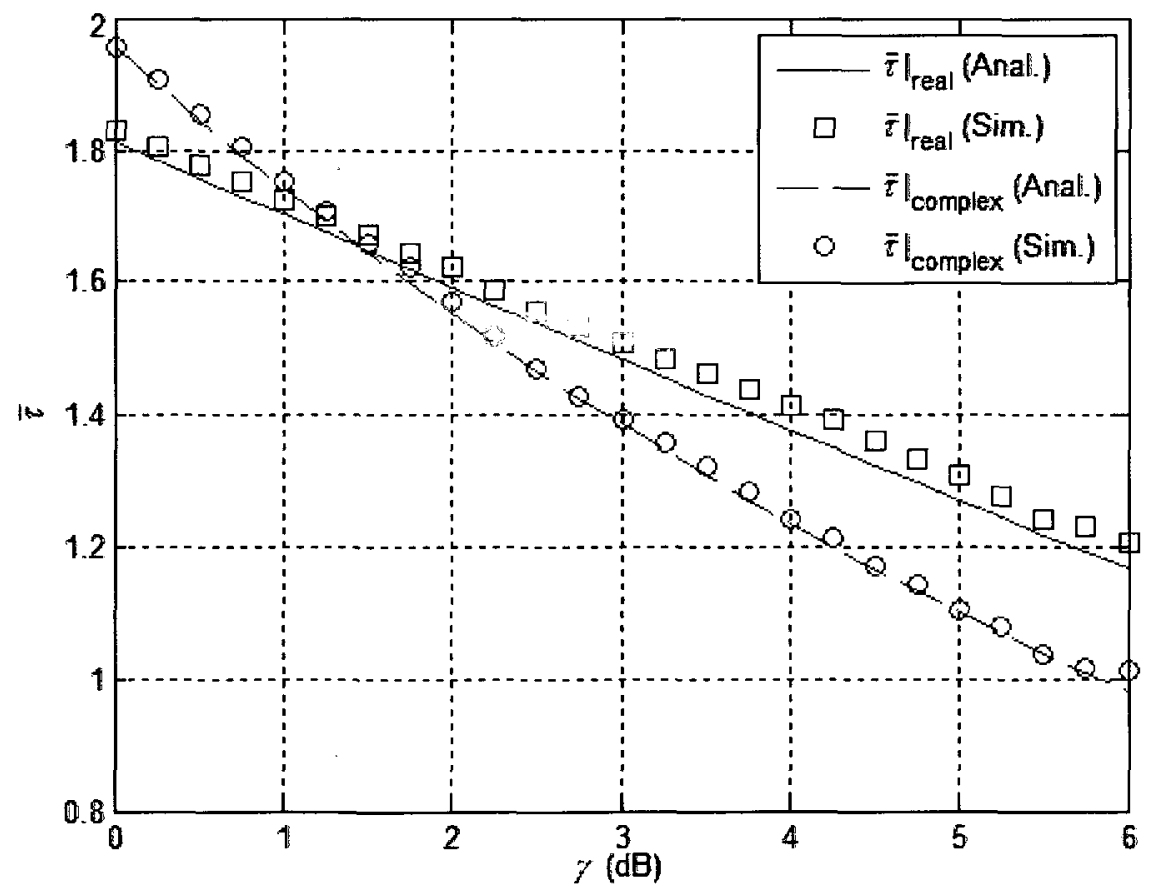

Fig. 4.5 Mean threshold crossing duration. 
To demonstrate the effect of the above-mentioned signal characteristics on spectral regrowth, different representations of 8-, 16-, and 64-user CDMA signals with the same variance $\left(\sigma_{x}^{2}=1\right)$ and Walsh codes of length $L=64$ are generated. An SRRC filter is used with roll-off and upsampling factor of 0.22 and 4, respectively. It is worth investigating whether such characteristics have the same effect on spectral regrowth, or is there one that is more dominant than the others. Actually, no mathematical clue is available as yet; however, extensive simulations have shown that the threshold crossing rate is the most dominant as will be demonstrated shortly. This seems to be logical and acceptable, since for other characteristics (clipped portion variance and mean duration of threshold crossing events) to occur, there should already be a corresponding threshold crossing.

Spectral regrowth itself has no unique definition. Spectral regrowth can have different definitions depending on the system requirements and the application's specifications. Such specifications could be the range of frequencies that are considered as out-of-band based on the channel separation and the guard bands, or the allowable amount of out-of-band power to ensure no impact to neighboring devices/channels. Hence, the adjacent channel power ratio (ACPR) is often used as one possible measure for spectral regrowth. Since the PSD of the baseband CDMA signal is symmetric about the origin, we use the ACPR in the upper channel, which is defined as the ratio of the power in the main channel to the power in the upper adjacent channel [67] 


$$
A C P R_{\mathrm{UPPER}}(\mathrm{dB})=10 \log _{10}\left(\frac{\int_{B / 2}^{\infty} S(\omega) d \omega}{\int_{-B / 2}^{B / 2} S(\omega) d \omega}\right)
$$

Spectral regrowth due to the nonlinear amplifier chain is not the only cause of adjacent channel interference, since the input signal to the amplifier could also have different amounts of adjacent channel interference caused by other factors such as filtering and modulation. Thus, to have a fair comparison, the signal representations are generated such that they have the same $A C P R_{i}$ before the amplifier.

The PSDs of the input, output, and clipped portions for all representations are evaluated at different IBO values and plotted in Fig. 4.6, Fig. 4.7, and Fig. 4.8. Fig. 4.6 shows the PSDs of the representations R1-R4 of an 8-user CDMA signal, Fig. 4.7 shows the PSDs of the representations R5-R8 of a 16-user CDMA signal, and Fig. 4.8 shows the PSDs of the representations R9-R12 of a 64-user CDMA signal. 


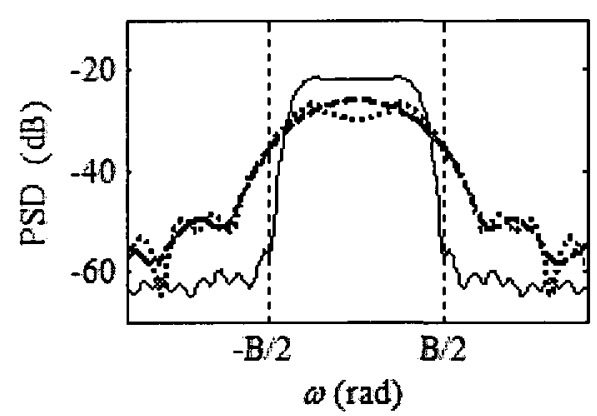

(a)

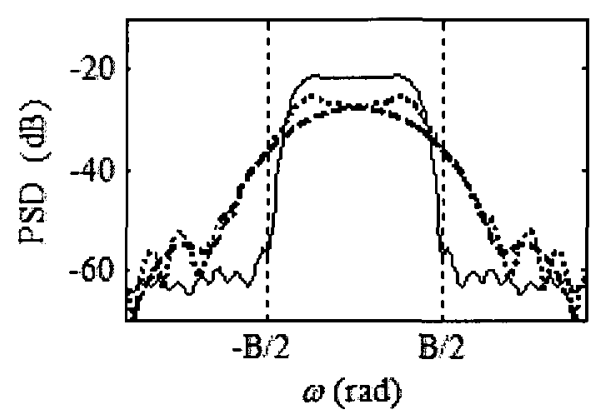

(c)

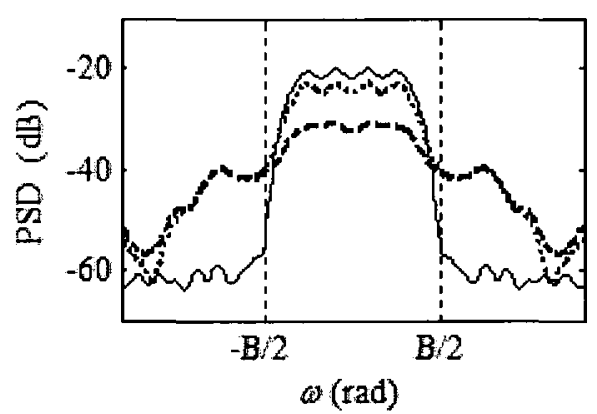

(b)

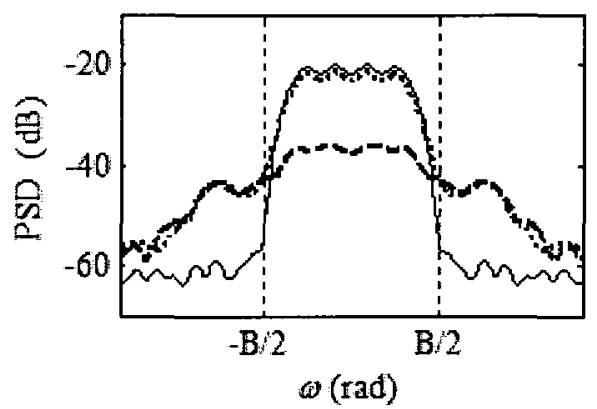

(d)

Fig. 4.6 (a)-(d) PSDs for representations R1-R4 of 8-user CDMA signal: the solid line represents input linear PSD $S_{x x}(\omega)$, the dotted line represents the output distorted PSD $S_{x_{d} x_{d}}(\omega)$, and the dashed line represents the PSD of the clipped signal portion $S_{x_{c} x_{c}}(\omega)$. 


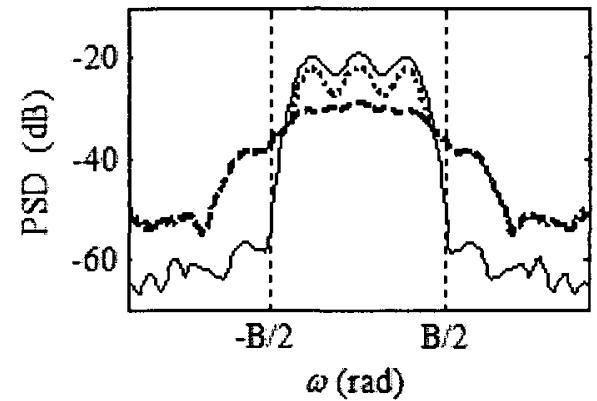

(a)

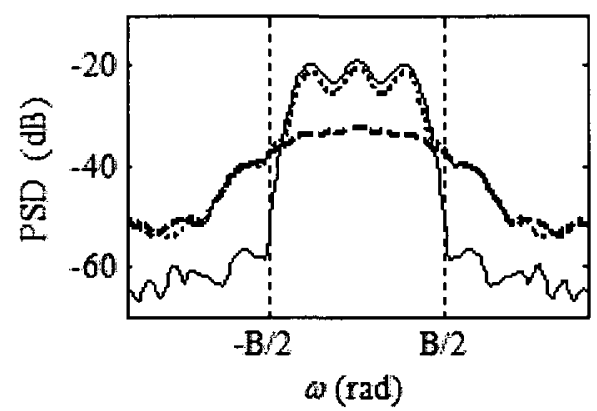

(c)

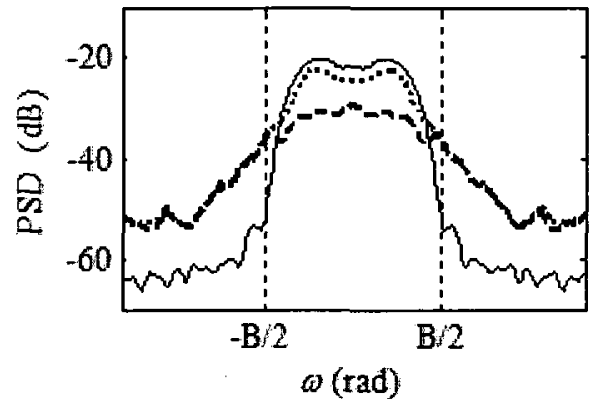

(b)

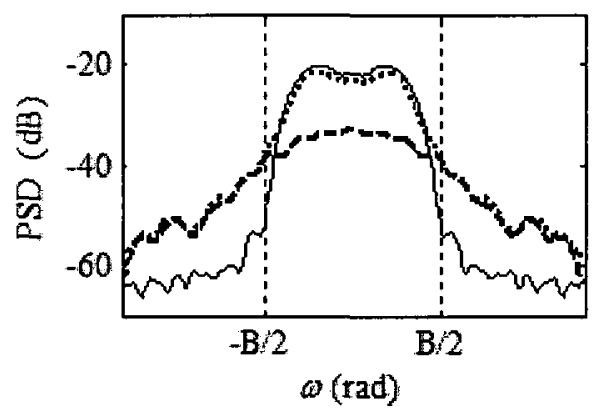

(d)

Fig. 4.7 (a)-(d) PSDs for representations R5-R8 of 16-user CDMA signal: the solid line represents input linear PSD $S_{x x}(\omega)$, the dotted line represents the output distorted PSD $S_{x_{d} x_{d}}(\omega)$, and the dashed line represents the PSD of the clipped signal portion $S_{x_{c} x_{c}}(\omega)$. 


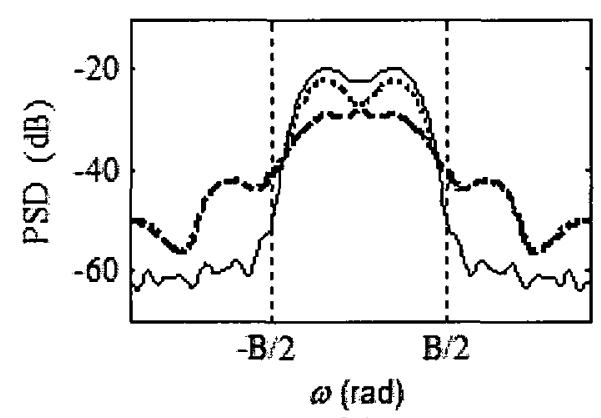

(a)

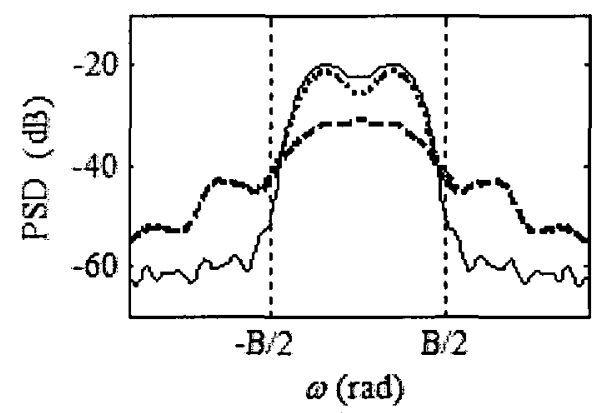

(c)

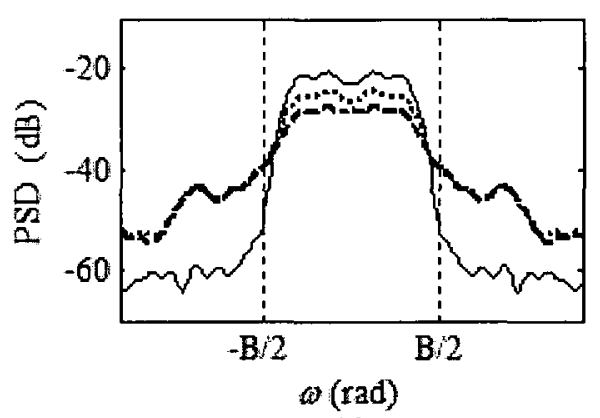

(b)

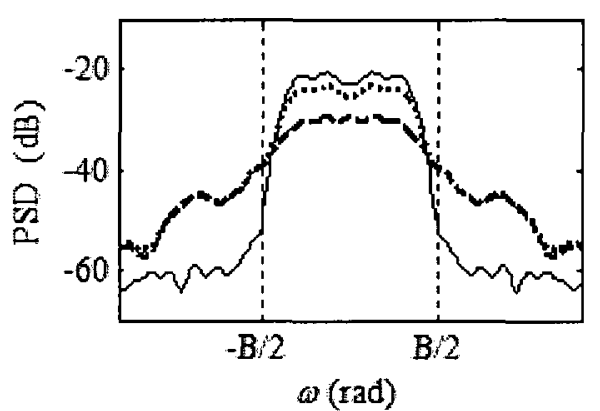

(d)

Fig. 4.8 (a)-(d) PSDs for representations R9-R12 of 64-user CDMA signal: the solid line represents input linear PSD $S_{x x}(\omega)$, the dotted line represents the output distorted PSD $S_{x_{d} x_{d}}(\omega)$, and the dashed line represents the PSD of the clipped signal portion $S_{x_{c} x_{c}}(\omega)$.

Looking to Fig. 4.6-4.8, it should be clear that the PSD of the distorted output signal almost coincides with the PSD of the clipped signal portions in the adjacent channels for all representations. This agrees with (4.13) that the PSD of the clipped signal portion is the primary component responsible for the additional out-of-band emissions due to the PD-HPA, i.e., spectral regrowth. 
The threshold crossing rate $R_{C}$, the variance of the clipped signal portion $\sigma_{c}^{2}$, the mean threshold crossing duration $\bar{\tau}$, and the ACPR of the distorted output signal $A C P R_{0}$ are calculated at different IBO levels $\gamma$ for each representation and tabulated in Tables $4.1-4.3$, respectively.

Table 4.1 Signal characteristics for 8-user DS-CDMA signal representations.

\begin{tabular}{|l|c|c|c|c|c|c|}
\hline & $A C P R_{i}(\mathrm{~dB})$ & $\gamma(\mathrm{dB})$ & $R_{C}$ & $\sigma_{c}^{2}$ & $\bar{\tau}(\mathrm{s})$ & $A C P R_{o}(\mathrm{~dB})$ \\
\hline $\mathrm{R} 1$ & -40.86 & 1 & 0.06 & 0.162 & 2.5 & -24.81 \\
\hline R2 & -40.86 & 1 & 0.17 & 0.056 & 2.3 & -21.75 \\
\hline R3 & -40.86 & 3 & 0.06 & 0.114 & 2.4 & -27.79 \\
\hline R4 & -40.86 & 3 & 0.17 & 0.019 & 1.5 & -26.08 \\
\hline
\end{tabular}

Table 4.2 Signal characteristics for 16-user DS-CDMA signal representations.

\begin{tabular}{|l|c|c|c|c|c|c|}
\hline & $A C P R_{i}(\mathrm{~dB})$ & $\gamma(\mathrm{dB})$ & $R_{C}$ & $\sigma_{c}^{2}$ & $\bar{\tau}(\mathrm{s})$ & $A C P R_{o}(\mathrm{~dB})$ \\
\hline R5 & -41.58 & 1 & 0.14 & 0.144 & 2.0 & -24.00 \\
\hline R6 & -41.58 & 1 & 0.14 & 0.139 & 1.8 & -24.36 \\
\hline R7 & -41.58 & 3 & 0.14 & 0.076 & 1.3 & -25.66 \\
\hline R8 & -41.58 & 3 & 0.11 & 0.078 & 1.5 & -28.31 \\
\hline
\end{tabular}

Table 4.3 Signal characteristics for 64-user DS-CDMA signal representations.

\begin{tabular}{|l|c|c|c|c|c|c|}
\hline & $A C P R_{i}(\mathrm{~dB})$ & $\gamma(\mathrm{dB})$ & $R_{C}$ & $\sigma_{c}^{2}$ & $\bar{\tau}(\mathrm{s})$ & $A C P R_{o}(\mathrm{~dB})$ \\
\hline R9 & -40.00 & 1 & 0.13 & 0.080 & 1.85 & -23.23 \\
\hline R10 & -40.00 & 1 & 0.15 & 0.115 & 1.85 & -22.39 \\
\hline R11 & -40.00 & 3 & 0.08 & 0.045 & 1.65 & -24.81 \\
\hline R12 & -40.00 & 3 & 0.09 & 0.075 & 1.55 & -24.62 \\
\hline
\end{tabular}


From the results in Tables 4.1-4.3, it can be concluded that the threshold crossing rate $R_{C}$ is the main contributor to spectral regrowth and that reducing $R_{C}$ reduces the output ACPR. Also, if two or more representations have almost the same number of threshold crossings, other characteristics start to play more of a role in controlling the out-of-band emissions. For instance, (R1, R3), (R2, R4), (R5, R6, R7), (R9, R10), and (R11, R12) have almost the same number of threshold crossings. In this case, the representation with the smaller clipped signal portion variance has a lower $A C P R_{o}$. Therefore, the results of the simulation experiments agree with our analysis that the main contributors to spectral regrowth due to the nonlinear PD-HPA are the threshold crossings rate $R_{C}$, the variance of the signal portion exceeding the PD-HPA clipping threshold $\sigma_{c}^{2}$, and the mean threshold crossing duration $\bar{\tau}$. Moreover, the usefulness of establishing such contributors clearly appears in the cases of small number of users. For instance, in (R1, R2) and (R7, R8), an attenuation of about $3 \mathrm{~dB}$ in $A C P R_{o}$ is achieved just by finding other representations with different signal characteristics. Also, it is clear that when the number of users is high, the signal characteristics are very close, since the CDMA signal approaches a Gaussian distribution, which is fixed. In this case, finding different representations with different noticeable characteristics is difficult. Therefore, the suggested solution to have different characteristics that lead to lower spectral regrowth is to increase the IBO level thru the developed expressions in (4.25), (4.28), (4.35), (4.26), (4.32), and (4.36). 


\subsection{Special case: spectral regrowth reduction in CDMA systems with orthogonal codes}

In our previous analysis, we were dealing with spreading codes in general. In this section, we study CDMA signals with orthogonal spreading codes as a special case. It will be shown that the PSD of orthogonal CDMA signals depends primarily on the PSDs of the assigned codes to each user in the system. And hence, based on the specific properties of the spreading codes used, we would be able to reduce the amount of the outof-band emissions and, in turn, reduce the spectral regrowth at the HPA output. The orthogonal spreading codes considered herein are the Walsh codes.

\subsubsection{PSD analysis for CDMA signals with orthogonal codes}

In this subsection, we study the dependence of the CDMA signal, particularly the PSD, on the spreading function assigned to each user in the system. For a synchronous CDMA system with equal users' power, the complex envelope of the CDMA symbol is given by

$$
s(t)=\sum_{k=1}^{K} s_{k}(t)=\sum_{k=1}^{K} \sqrt{E_{k}} a_{n}^{(k)} c^{(k)}(t)
$$


Since $s(t)$ is assumed to be stationary, the notation can be simplified by introducing subscripts to indicate quantities at different times as follows.

$$
\begin{gathered}
s_{\tau}(t)=s(t+\tau) \\
c_{\tau}^{(k)}(t)=c^{(k)}(t+\tau) .
\end{gathered}
$$

Using (4.38) and the simplified notations, the autocorrelation function of the CDMA signal can be expanded as

$$
\begin{aligned}
R_{s s}(\tau)= & E\left\{s(t) s^{*}(t+\tau)\right\}=E\left\{s_{0} s_{\tau}^{*}\right\} \\
=E & \left\{\left(\sum_{k=1}^{K} \sqrt{E_{k}} a_{n}^{(k)} c_{0}^{(k)}\right) \cdot\left(\sum_{m=1}^{K} \sqrt{E_{m}} a_{n}^{(m)} c_{\tau}^{(m)}\right)^{*}\right\} \\
=E_{k} \cdot E\left\{\left|a_{n}^{(1)}\right|^{2} c_{0}^{(1)} c_{\tau}^{(1)}+a_{n}^{(1)} a_{n}^{(2)^{*}} c_{0}^{(1)} c_{\tau}^{(2)}+\cdots+a_{n}^{(1)} a_{n}^{(K)^{*}} c_{0}^{(1)} c_{\tau}^{(K)}\right. & \\
& +a_{n}^{(2)} a_{n}^{(1)^{*}} c_{0}^{(2)} c_{\tau}^{(1)}+\left|a_{n}^{(2)}\right|^{2} c_{0}^{(2)} c_{\tau}^{(2)}+\cdots+a_{n}^{(2)} a_{n}^{(K)^{*}} c_{0}^{(2)} c_{\tau}^{(K)} \\
& +\cdots \\
& \left.+a_{n}^{(K)} a_{n}^{(1)^{*}} c_{0}^{(K)} c_{\tau}^{(1)}+a_{n}^{(K)} a_{n}^{(2)}{ }^{*} c_{0}^{(K)} c_{\tau}^{(2)}+\cdots+\left|a_{n}^{(K)}\right|^{2} c_{0}^{(K)} c_{\tau}^{(K)}\right\} .
\end{aligned}
$$

Assuming perfect synchronization and neglecting the partial cross-correlation for simplicity, the Walsh code functions have approximately zero cross-correlation [68]. That is 


$$
R_{c^{(k)} c^{(m)}}(\tau)=E\left\{c_{0}^{(k)} c_{\tau}^{(m)}\right\} \cong\left\{\begin{aligned}
R_{c c}^{(k)}(\tau), & k=m \\
0, & k \neq m
\end{aligned}\right.
$$

With such zero cross-correlations between the Walsh code functions and using the fact that the data symbols $a_{n}^{(k)}$ are independent of the Walsh code functions, (4.40) reduces to

$$
\begin{aligned}
R_{s s}(\tau) \cong E_{k} \cdot( & E\left\{\left|a_{n}^{(1)}\right|^{2}\right\} R_{c c}^{(1)}(\tau) \\
& +E\left\{\left|a_{n}^{(2)}\right|^{2}\right\} R_{c c}^{(2)}(\tau)+\cdots \\
& \left.+E\left\{\left|a_{n}^{(K)}\right|^{2}\right\} R_{c c}^{(K)}(\tau)\right)
\end{aligned}
$$

Moreover, with the assumption that the symbols are $a_{n}^{(k)}$ are statistically i.i.d with zero mean and variance $E\left\{\left|a_{n}^{(k)}\right|^{2}\right\}=P, \forall k, P$ is constant depending on the modulation format adopted, the autocorrelation function of the CDMA signal can be written as

$$
R_{s s}(\tau) \cong E_{k} \cdot P \sum_{k=1}^{K} R_{c c}^{(k)}(\tau)
$$

Finally, the PSD of the CDMA signal is obtained as the Fourier transform of the autocorrelation function,

$$
S_{s s}(f)=\int_{-\infty}^{\infty} R_{s s}(\tau) e^{-j 2 \pi f \tau} d \tau
$$




$$
\begin{aligned}
& \cong E_{k} \cdot P \sum_{k=1}^{K} \int_{-\infty}^{\infty} R_{c c}^{(k)}(\tau) e^{-j 2 \pi f \tau} d \tau \\
& \cong E_{k} \cdot P \sum_{k=1}^{K} S_{c c}^{(k)}(f) .
\end{aligned}
$$

From (4.44), it is clear that the PSD of a synchronous CDMA signal, with orthogonal spreading codes, is approximately a scaled sum of the PSDs of the Walsh codes assigned to each user in the system. This relation is demonstrated in Fig. 4.9 for three different Walsh code assignments, where three representations of an 8-user CDMA signal with three different Walsh code sets of length $L=64$ are generated. An SRRC filter with an upsampling factor of 4 and 0.22 roll-off factor is used. Let $W_{m}=$ $\left\{w_{i}^{(k)}\right\}, i=0, \ldots, L-1, k=1, \ldots, K$ denote the Walsh set for the $m$ th representation where $w_{i}^{(k)}$ is the Walsh function assigned to the $k$ th user, which corresponds to the $i$ th row of a Hadamard matrix of size $L \times L$. For the examples in Fig. 4.9, the three different Walsh code sets are as follows:

$$
\begin{aligned}
& W_{1}=\left\{w_{0}, w_{1}, w_{2}, w_{3}, w_{4}, w_{5}, w_{6}, w_{7}\right\} \\
& W_{2}=\left\{w_{0}, w_{3}, w_{8}, w_{9}, w_{11}, w_{16}, w_{24}, w_{30}\right\} \\
& W_{3}=\left\{w_{0}, w_{6}, w_{10}, w_{24}, w_{36}, w_{42}, w_{49}, w_{59}\right\}
\end{aligned}
$$




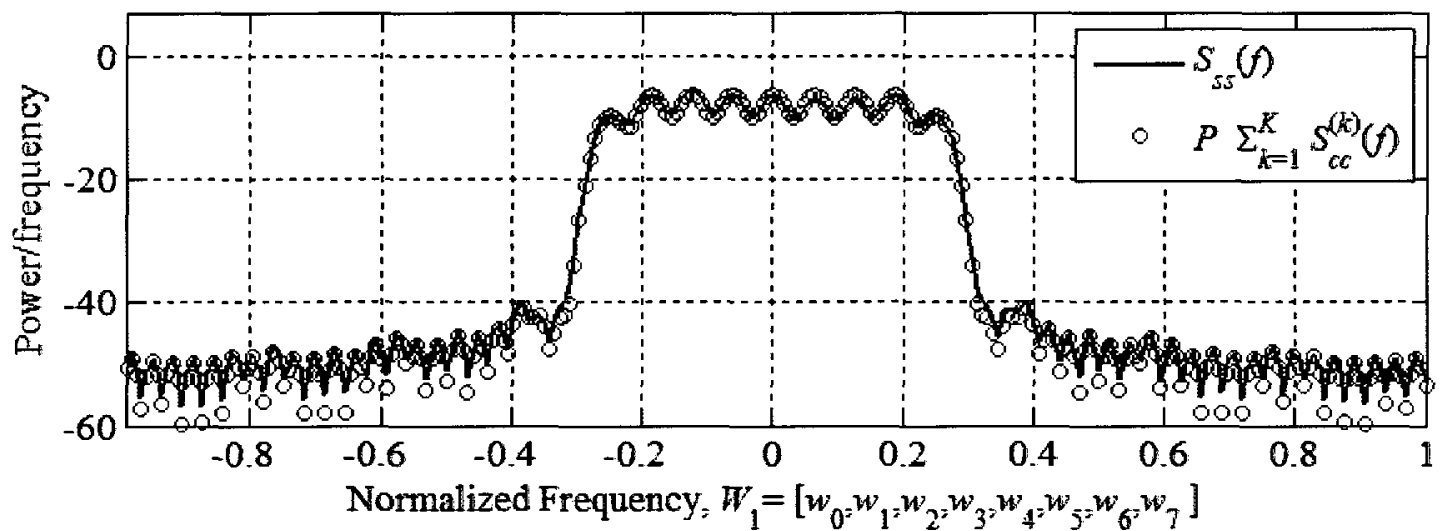

(a)

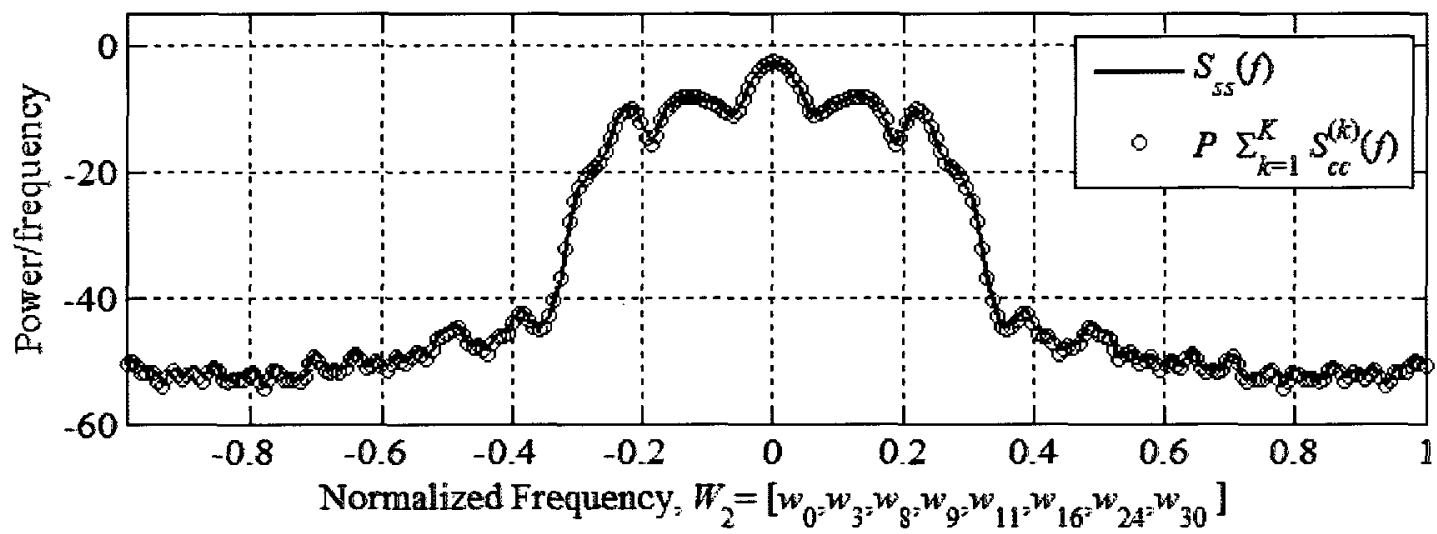

(b)

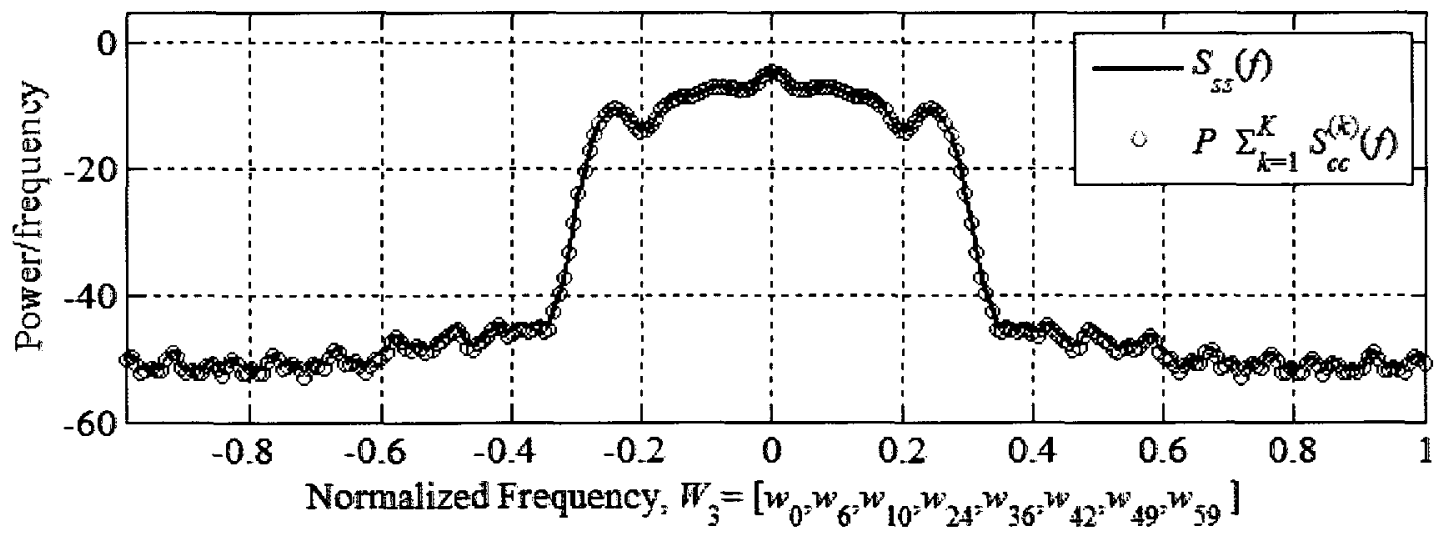

(c)

Fig. 4.9 PSD plots for an 8-user CDMA signal with three different Walsh code assignments; (a) $W_{1}$, (b) $W_{2}$, and (c) $W_{3}$. 
The solid lines in Fig. 4.9 (a)-(c) show the PSDs of the three representations with the code sets $W_{1}, W_{2}$, and $W_{3}$, respectively. The sums of the PSDs of the Walsh functions $\left\{w_{i}\right\}$ in each Walsh code set $\left(W_{1}, W_{2}\right.$, and $\left.W_{3}\right)$ are also plotted with circle markers. From the curves, it is clear that the PSDs of the CDMA signals are nearly identical to the scaled sum of the PSDs of the Walsh codes assigned to each user, which agrees with (4.44).

\subsubsection{Walsh code properties}

In the previous section, we have shown that the PSD of a synchronous CDMA signal with orthogonal Walsh spreading codes depends primarily on the PSDs of the Walsh functions assigned to the users in the system. In this section, we study the dependency of the PSD of the Walsh functions on the Walsh code properties. Through the investigation of Walsh codes, we find that each Walsh codeword has its own PSD shape depending on the maximum runlength. The maximum runlength is defined as the maximum number of successive similar elements in the codeword, i.e., the maximum number of continuous +1 's or -1 's. The set of all Walsh codewords of length $L=8$ are shown in Fig. 4.10. An important property regarding the relation between the runlength $r_{i}$ of a Walsh codeword of length $L$ and its corresponding index $i$ is

$$
r_{i}= \begin{cases}L, & i=0 \\ 1, & i=1 \\ 2^{b+1}, & i>1\end{cases}
$$



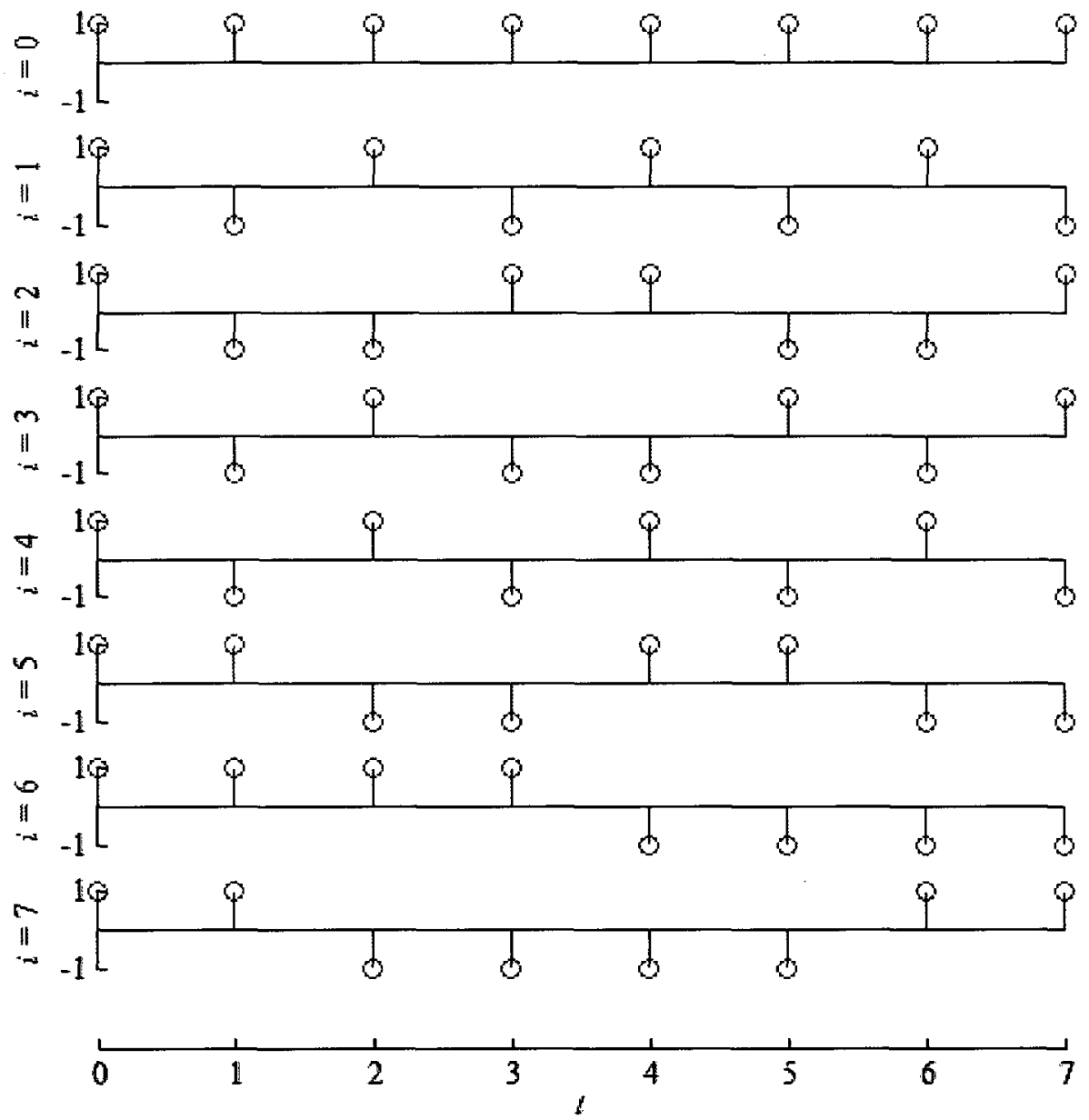

Fig. 4.10 Walsh codewords of length $L=8$.

In (4.46),

$$
b=\log _{2}\left(\max _{\beta \in\{0, \cdots, M-1\}} \operatorname{gcd}\left(i, 2^{\beta}\right)\right),
$$

where

$$
M=\max \left(1,\left[\log _{2} i\right]\right) .
$$


and $\operatorname{gcd}(\cdot, \cdot)$ determines the greatest common divisor.

Based on (4.46), two useful rules regarding the PSD of Walsh codewords and their sums are provided:

1. The higher the value of $b$ in a Walsh codeword with index a multiple of $2^{b}$, the more the power is concentrated around the centre frequency in its PSD.

2. In a set of Walsh codewords, the higher the number of Walsh codewords whose index is a multiple of $2^{b}$, especially for $b \geq 3$, the more the power is concentrated around the centre frequency in the PSD of the set sum.

To illustrate the above rules, the PSDs of three different sets of Walsh codes after using the SRRC filter with roll-off of 0.22 and upsampling factor of 4 , are plotted in Fig. 4.11. The following sets of Walsh codes are used:

$$
\begin{aligned}
& W_{1}=\left\{w_{0}, w_{1}, w_{2}, w_{3}, w_{4}, w_{5}, w_{6}, w_{7}\right\}, \\
& W_{2}=\left\{w_{0}, w_{1}, w_{4}, w_{8}, w_{11}, w_{16}, w_{24}, w_{48}\right\}, \text { and } \\
& W_{3}=\left\{w_{0}, w_{8}, w_{16}, w_{24}, w_{32}, w_{40}, w_{48}, w_{56}\right\} .
\end{aligned}
$$

The PSD is calculated using Welch's method with parameters of a Hamming window with segment length of 40 samples and $50 \%$ overlap between segments. 


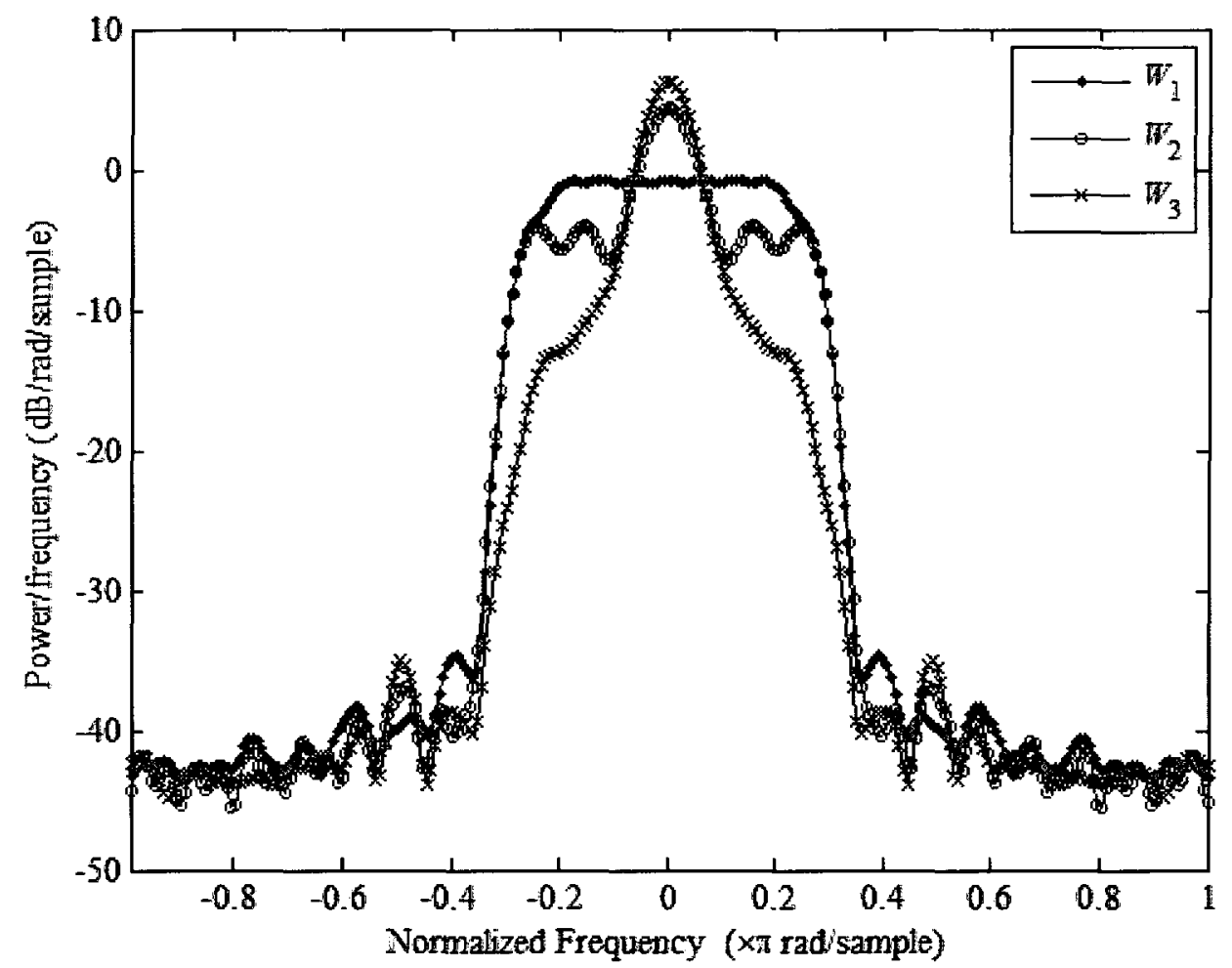

Fig. 4.11 PSD plots of different Walsh code sets with SRRC filtering.

From the above discussion, it is clear that by selecting certain Walsh code sets having Walsh functions of indices equal to a multiple of $2^{b}$ ( $b$ is as large as possible), we achieve a narrower bandwidth. For the example shown in Fig. 4.11, we see that all the codewords in the third code set are multiple of $2^{b}$ compared to one codeword $\left(w_{0}\right)$ and six codewords $\left(w_{0}, w_{4}, w_{8}, w_{16}, w_{24}, w_{48}\right)$ in the first and the second code sets, respectively. Consequently, as can be seen in Fig. 4.11, as the number of the codewords of multiple of $2^{b}$ in the code set increases, the signal bandwidth decreases. This decrease in bandwidth 
allows more room in the main channel for power leakage when the signal is applied to a nonlinear amplifier chain.

\subsubsection{Walsh code assignment algorithm}

Walsh codes assignment have grabbed much attention as a tool for reducing the effect of HPA nonlinearity in CDMA systems such as $[20,69,70]$. Different parameters have been adopted as measures of the signal's sensitivity to the nonlinear amplifications, upon which the assignment is performed.

In [20], PAR was adopted as the nonlinearity measure, where the author described that within the set of Walsh codes in the system, there exists some Walsh codes that lead to minimum PARs and others that lead to higher PARs. So, as new users are added to the system, they are assigned Walsh codes so as to keep a minimal PAR. In [69], based on the properties of Walsh codes and the relation between Walsh codes' runlengths and their indices, the authors introduced an algorithm for Walsh code assignment to reduce the PAR. However, as Lau [20] observed, the PAR cannot always be taken as the nonlinearity measure since, in cases of large number of users, the pdf of the CDMA signal approaches a Gaussian distribution, which is fixed, and hence the differences between PARs among different representations tend to vanish. Therefore, the introduced techniques of Walsh codes assignment for PAR reduction work primarily for small number of users. 
The power variance was proposed as a measure of the signal's sensitivity to the HPA nonlinearity in [70]. In [70], it was shown that each code of an $L$-Walsh code set can be represented as the product of $\log _{2} L$ (or fewer) Hadamard basis functions, where, for instance, the basis functions of a $64-$ Walsh codes are: $w_{0}, w_{1}, w_{2}, w_{4}, w_{8}, w_{16}, w_{32}$. The author established, based on such a fact, that as long as the selected Walsh codes have a larger representation of the basis functions, especially $w_{1}$ and $w_{2}$, the power variance will be small.

In our scenario, our focus is to reduce spectral regrowth caused by the amplifier nonlinearity. Hence, we present an effective Walsh code assignment algorithm to reduce spectral regrowth, based on two facts. First, the PSD of the synchronous CDMA signal depends primarily on the sum of individual PSDs of the Walsh codes assigned to the users. Second, the Walsh code properties given in Rules 1 and 2 described in Section 4.4.2.

The base station maintains the bins $\left\{v_{b}\right\}, b=0, \ldots, M-1$, where $M=\log _{2} L$ and $L$ is the Walsh code length. First, bin $v_{M-1}$ is filled with $w_{0}$ then each bin, $v_{b}$, is filled with every Walsh code with index $i$ according to (4.47) and (4.48). Then, the base station starts assigning Walsh functions for traffic and overhead channels starting from the bin with the highest value of $b$. At each new call origination, the base station looks at the remaining highest bin that has an available Walsh code to assign to the new user. Fig. 4.12 illustrates the setup of the Walsh code assignment algorithm for $L=64$. 


\begin{tabular}{|c|c|c|c|c|c|c|c|c|c|c|c|c|c|c|c|c|}
\hline$v_{5}$ & \multicolumn{16}{|c|}{$w_{0}$} \\
\hline$v_{4}$ & \multicolumn{8}{|c|}{$w_{32}$} & \multicolumn{8}{|c|}{$w_{48}$} \\
\hline$v_{3}$ & \multicolumn{4}{|c|}{$w_{16}$} & \multicolumn{4}{|c|}{$w_{24}$} & \multicolumn{4}{|c|}{$w_{40}$} & \multicolumn{4}{|c|}{$w_{56}$} \\
\hline$v_{2}$ & \multicolumn{2}{|c|}{$w_{8}$} & \multicolumn{2}{|c|}{$w_{12}$} & \multicolumn{2}{|c|}{$w_{20}$} & \multicolumn{2}{|c|}{$w_{28}$} & \multicolumn{2}{|c|}{$w_{36}$} & \multicolumn{2}{|c|}{$w_{44}$} & \multicolumn{2}{|c|}{$w_{52}$} & \multicolumn{2}{|c|}{$w_{60}$} \\
\hline$v_{I}$ & $w_{4}$ & $w_{6}$ & $w_{10}$ & $w_{14}$ & $w_{18}$ & $w_{22}$ & $w_{26}$ & $w_{30}$ & $w_{34}$ & $w_{38}$ & $w_{42}$ & $w_{46}$ & $w_{50}$ & $w_{54}$ & $w_{58}$ & $w_{62}$ \\
\hline$v_{0}$ & $w_{2}$ & $w_{1}$ & $w_{3}$ & $w_{5}$ & $w_{7}$ & $w_{9}$ & & & 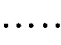 & $\ldots$ & D & 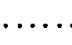 & & & $w_{61}$ & $w_{63}$ \\
\hline
\end{tabular}

Fig. 4.12 Walsh code assignment algorithm for $L=64$.

\subsubsection{Performance assessment of the Walsh code assignment algorithm}

In this section, we assess the performance of the Walsh code assignment algorithm to effectively reduce spectral regrowth due to the nonlinear amplifier chain. The ACPR is used as a measure for spectral regrowth following the expression of the upper ACPR in (4.37).

In assessing the performance of the Walsh code assignment algorithm, we consider the same scenario as in [69] with 14 active traffic channels and 3 overhead channels. The overhead channels are the pilot, paging and sync channels. Three representations for a CDMA signal with Walsh code length of $L=64$ are generated. An SRRC pulse shaping filter is used with roll-off factor 0.22 and upsampling factor of 4. The Walsh code sets used for three representations are as follows: 


$$
\begin{aligned}
& W_{1}=\left\{w_{0}, w_{1}, w_{2}, w_{3}, w_{4}, w_{5}, w_{6}, w_{7} w_{8}, w_{9}, w_{10}, w_{11}, w_{12}, w_{13}, w_{14}, w_{15}, w_{32}\right\} \\
& W_{2}=\left\{w_{0}, w_{1}, w_{2}, w_{6}, w_{10}, w_{16}, w_{20}, w_{28}, w_{30}, w_{32}, w_{34}, w_{38}, w_{42}, w_{48}, w_{52}, w_{54} w_{60}\right\}
\end{aligned}
$$

and the third set is built using the Walsh code assignment algorithm as

$$
W_{3}=\left\{w_{0}, w_{4}, w_{8}, w_{12}, w_{14}, w_{16}, w_{20}, w_{24}, w_{28}, w_{32}, w_{36}, w_{40}, w_{44}, w_{46}, w_{48}, w_{52}, w_{56}\right\}
$$

The ACPRs are calculated for the CDMA signal representations with these Walsh code sets in the presence of a PD-HPA at different IBOs. The results for the ACPR in the upper adjacent channels, in $\mathrm{dB}$, are tabulated in Table 4.4. From the table, it is clear that the CDMA signal representation whose Walsh codes are assigned according to the proposed Walsh assignment algorithm has the lowest $A C P R_{U P P E R}$ at all values of IBO,

\begin{tabular}{|c|c|c|c|c|}
\hline & \multicolumn{3}{|c|}{$\mathrm{ACPR}_{\text {UPPER }}$} \\
\hline & & $\overline{W_{1}}$ & $W_{2}$ & $\overline{W_{3}}$ \\
\hline \multirow{4}{*}{ 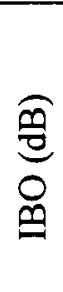 } & 1 & -24.4334 & -25.1743 & -30.4360 \\
\hline & 2 & -28.6523 & -29.4131 & -33.1910 \\
\hline & 3 & -33.6196 & -33.4546 & -36.1319 \\
\hline & 4 & -36.0161 & -37.1007 & -38.1273 \\
\hline \multicolumn{2}{|c|}{ Linear } & -36.3235 & -38.1975 & -38.4938 \\
\hline
\end{tabular}
particularly at lower IBOs.

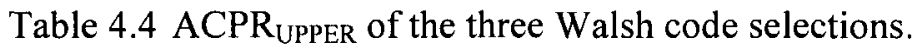


From the results depicted in Table 4.4, it is clear that the proposed Walsh code assignment algorithm achieves lower spectral regrowth in terms of ACPR at all values of IBO. It is worth noting that, at lower values of IBO, the spectral regrowth reduction is really remarkable. Also by looking to the results in Table 4.4 , one can observe that using the proposed algorithm offers a $1 \mathrm{~dB}$ gain in the IBO to achieve the same ACPR. This implies that using this algorithm the HPA can operate at high values of input power approaching the saturation level with minimal spectral regrowth and, in turn, the HPA efficiency is improved.

Fig. 4.13 shows the PSD for the CDMA signal with the three Walsh code sets in the presence of a PD-HPA at different values of IBOs. The PSD is calculated using Welch's method using a Hamming window with segment length of 64 samples and $50 \%$ overlap between segments. From the curves in Fig. 4.13, it is clear that the representation with the Walsh code set selected using the proposed Walsh assignment algorithm has lower spectral regrowth as compared to the other two representations with different Walsh code sets. 


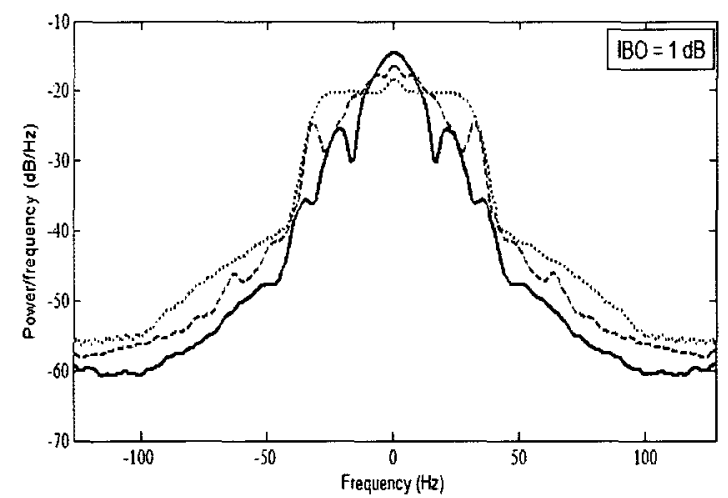

(a)

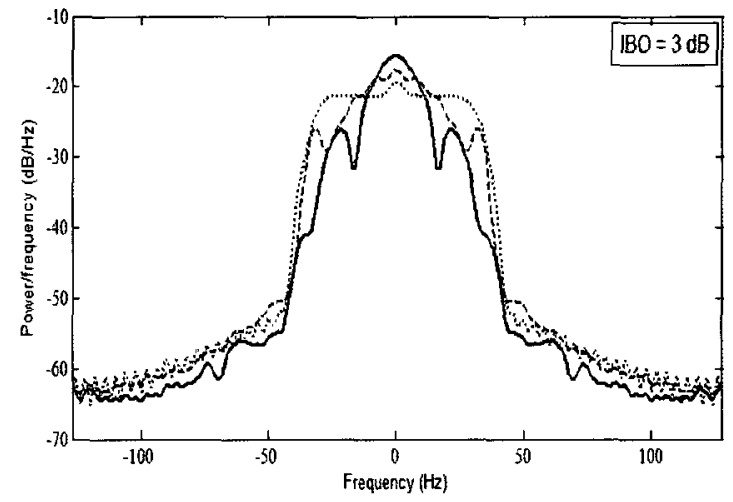

(c)

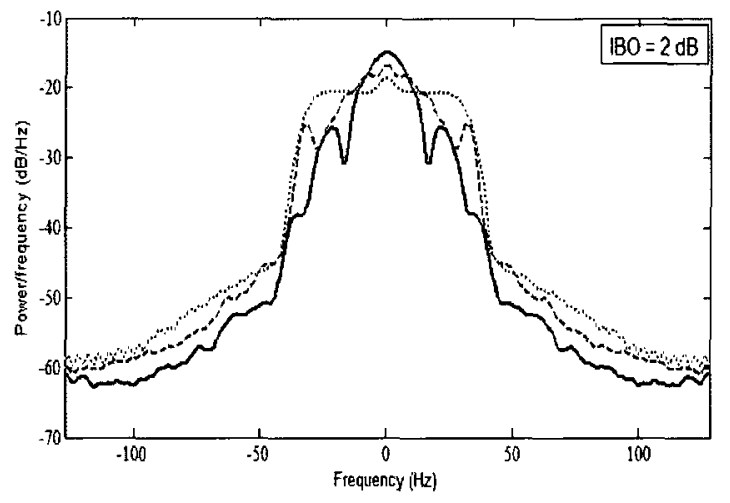

(b)

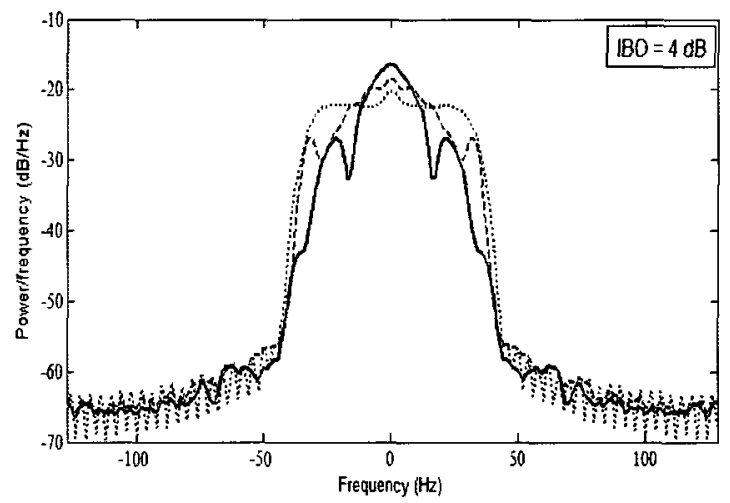

(d)

Fig. 4.13 PSD plots in presence of PD-HPA at different IBOs (dB): $W_{1}$ dotted curve, $W_{2}$ dashed curve and $W_{3}$ solid curve: (a) $\mathrm{IBO}=1$, (b) $\mathrm{IBO}=2$, (c) $\mathrm{IBO}=3$, and (d) $\mathrm{IBO}=4$.

\subsection{Conclusions}

In this chapter, we analyzed the spectral regrowth in the transmitted signals caused by nonlinear amplification. Based on this analysis, we established which signal characteristics at the PD-HPA input mainly contribute to spectral regrowth. In relation to the PD-HPA saturation level, the input signal's threshold crossing rate $R_{C}$ and clipped signal variance $\sigma_{c}^{2}$ are correlated well with the expected spectral regrowth, with lower 
values in $R_{C}$ and $\sigma_{c}^{2}$ resulting in a lower $\mathrm{ACPR}_{o}$. In cases of input representations with similar $R_{C}$ and $\sigma_{c}^{2}$, the mean threshold crossing duration $\bar{\tau}$ provides additional discrimination between input representations to select the one with a lower $A C P R_{o}$. Based on the assumption that CDMA and OFDM signals are characterized as Gaussian processes for large number of users and subcarriers, respectively, analytical expressions for such characteristics were developed. Such an approach provides potential insight into how the CDMA signal characteristics and the HPA characteristics interact to produce spectral regrowth. Accordingly, one would be able to control such characteristics in order to minimize spectral regrowth before the signal hits the HPA using any of the available techniques such as SLM, PTS, precoding, and so forth.

Then, we investigated the CDMA signals with orthogonal spreading codes as a special case. It was shown that the PSD of orthogonal CDMA signals depends primarily on the PSDs of the assigned codes to each user in the system. Also, it was established that the PSD of a Walsh codeword and the PSD of the sum of Walsh codewords depend on the specific properties of the Walsh codes used, maximum runlength and indices. Based on such a trilateral relation among the PSD, maximum runlength, and indices, an efficient Walsh code assignment algorithm was proposed, with which Walsh code functions are assigned to the users so as to keep the spectral regrowth at the HPA output minimal. 


\section{Chapter 5}

\section{CHARACTERIZATION}

\section{OF NONLINEAR DISTORTION}

ver the decades, much research has been conducted trying to reduce the
vulnerability of the amplifier input signal to nonlinearity. Such research often seeks to define a measure for the nonlinear distortion, which when reduced often allows the nonlinear distortion to be also reduced. Several measures were adopted to quantify nonlinear distortion in relation to the input signal to the HPA. In this chapter, we first present a brief survey on the most currently known nonlinearity measures highlighting their advantages and their disadvantages. Then, we introduce an effective measure for characterizing the nonlinear distortion as an alternative to the existing measures, which is characterized by having a direct link to the system performance.

\subsection{Existing measures for nonlinear distortion characterization}

Several measures were adopted to quantify nonlinear distortion in relation to the input signal to the HPA. PAR is the most commonly used measure of the potential nonlinearity due to HPAs, which when reduced decreases the dynamic range of the input signal to the HPA $[20,21]$. In turn, the signal traverses a smaller range of the inherent nonlinearity of the HPA transfer function. Also, PAR allows the system designer to 
determine the required amount of IBO to achieve a certain acceptable performance. For a signal $s(t)$ of duration $T, E\{s(t)\}=0$, the PAR $\psi$ is defined as

$$
\psi=\frac{\max _{0 \leq t \leq T}|s(t)|^{2}}{E\left\{|s(t)|^{2}\right\}} .
$$

Recently, other measures have been adopted such as the instantaneous power variance (IPV) $[22,23,69,71,73]$ and the cubic metric (CM) $[22,24,72,73]$. The motivation for the IPV measure is to reduce the envelope fluctuations [73]. In order to remove the dependence on the average power, The IPV $\sigma_{|s|^{2}}^{2}$ is defined normalized to the average power as $[22,69]$

$$
\sigma_{|s|^{2}}^{2}=\frac{\operatorname{var}\left\{|s(t)|^{2}\right\}}{[\operatorname{var}\{s(t)\}]^{2}}=\frac{E\left\{|s(t)|^{4}\right\}}{\left[E\left\{|s(t)|^{2}\right\}\right]^{2}}
$$

The CM measure was proposed with the motivation of reducing the third-order modulation product as it is the cause of the major distortion [24]. The CM is defined as

$$
C M=\sqrt{E\left\{\left(\left|s_{\mathrm{rms}}(t)\right|^{3}\right)^{2}\right\}}
$$

While the existing measures have their use and have led to remarkable improvements, none of them has a close relation with the system performance. In other words, no clear mathematical relation exists between these measures and the resulting different forms of nonlinear distortion (BER degradation and spectral regrowth). 
Accordingly, their reduction may not lead to optimum performance. An example to illustrate the above idea is presented, where the BER is considered as the performance merit. Consider an example 16-user CDMA signal of duration $T$ with Walsh codes of length $L=64$ and filtered with an SRRC filter. Many representations of this signal have been generated. Three of them were selected in order to emphasize that the abovementioned measures do not always lead to optimum performance. The three representations $(\mathrm{R} 1, \mathrm{R} 2$, and $\mathrm{R} 3$ ) of such a signal are generated with the same average power $P_{a v}$ using the SLM technique [14]. Fig. 5.1 shows the instantaneous power of the three representations $r_{1}^{2}(t), r_{2}^{2}(t)$, and $r_{3}^{2}(t)$.
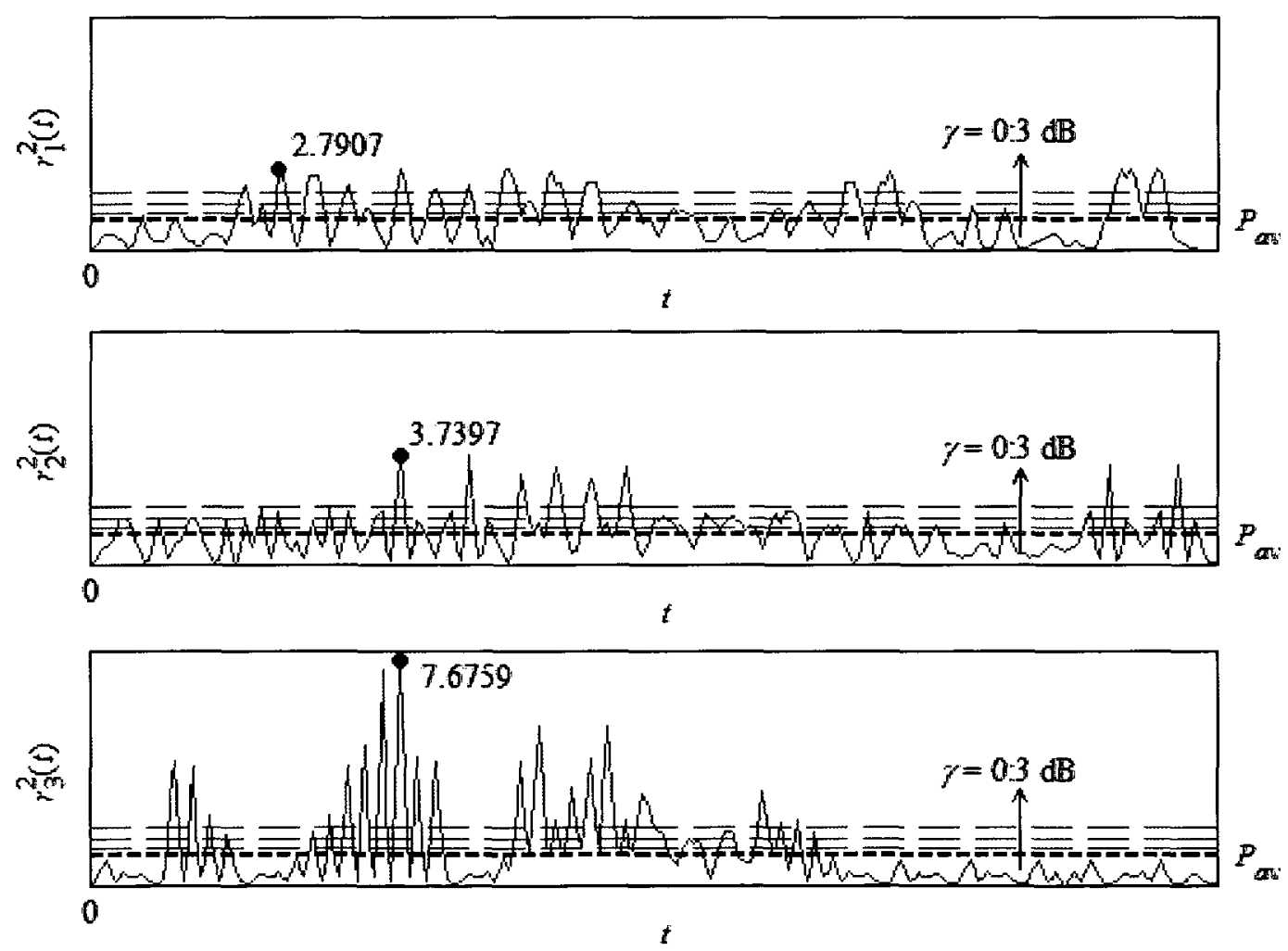

Fig. 5.1 Instantaneous powers $r_{1}^{2}(t), r_{2}^{2}(t)$, and $r_{3}^{2}(t)$ of $\mathrm{R} 1, \mathrm{R} 2$, and $\mathrm{R} 3$, respectively. 
The reader is reminded that it was established in Chapter 3 that the signal characteristics contributing to BER degradation caused by amplifier nonlinearity are: the threshold exceeding rate $R_{E}$ and the variance of the clipped signal portion $\sigma_{c}^{2}$. The PAR, IPV, and CM are calculated for each representation. Also $R_{E}$, and $\sigma_{c}^{2}$ are measured for each representation at different values of IBO $\gamma$ and tabulated in Table 5.1.

Table 5.1 Signal characteristics of representations R1, R2, and R3.

\begin{tabular}{|c|c|c|c|c|c|}
\hline & $\mathbf{R} 1$ & $\mathbf{R 2}$ & R3 & & \\
\hline $\operatorname{PAR}(\mathrm{dB})$ & 4.46 & 5.73 & 8.85 & & \\
\hline IPV & 0.61 & 0.51 & 1.51 & & \\
\hline $\mathrm{CM}$ & 0.36 & 0.36 & 0.12 & & \\
\hline \multirow{4}{*}{$R_{E}$} & 0.432 & 0.489 & 0.337 & 0 & \multirow{12}{*}{ 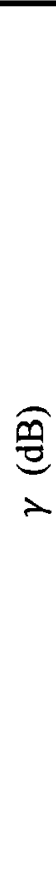 } \\
\hline & 0.280 & 0.341 & 0.322 & 1 & \\
\hline & 0.246 & 0.164 & 0.212 & 2 & \\
\hline & 0.140 & 0.083 & 0.155 & 3 & \\
\hline \multirow{4}{*}{$\sigma_{c}^{2}$} & 0.058 & 0.125 & 0.048 & 0 & \\
\hline & 0.027 & 0.033 & 0.142 & 1 & \\
\hline & 0.015 & 0.015 & 0.097 & 2 & \\
\hline & 0.006 & 0.004 & 0.063 & 3 & \\
\hline \multirow{4}{*}{ BER } & 0.137 & 0.146 & 0.129 & 0 & \\
\hline & 0.124 & 0.129 & 0.131 & 1 & \\
\hline & 0.121 & 0.115 & 0.121 & 2 & \\
\hline & 0.113 & 0.109 & 0.116 & 3 & \\
\hline
\end{tabular}


It is clear from Table 5.1 that, among the three representations, $\mathrm{R} 1$ has the minimum PAR, R2 has the minimum IPV, and R3 has the minimum $\mathrm{CM}$. Looking to the calculated parameters in Table 5.1, it can be observed that a representation with a minimum of one of the considered measures might have higher values of $R_{E}$ and $\sigma_{c}^{2}$ at certain IBO values. Hence, such a representation, according to the analysis in Chapter 3, will be more vulnerable to nonlinear distortion leading to greater BER degradation. For instance, $\mathrm{R} 1$ that has the lowest PAR, has the lowest values of $R_{E}$ and $\sigma_{c}^{2}$ only at $\mathrm{IBO}=1$ $\mathrm{dB}$, while has higher values than those of R2 and R3 at other IBO values. Similarly, R2 that has the lowest IPV, has the lowest values of $R_{E}$ and $\sigma_{c}^{2}$ at IBO $=3 \mathrm{~dB}$ and $4 \mathrm{~dB}$, while has higher values than those of $\mathrm{R} 1$ and $\mathrm{R} 3$ at other IBO values. Also, R3 that has the lowest $\mathrm{CM}$, has the lowest values of $R_{E}$ and $\sigma_{c}^{2}$ at $\mathrm{IBO}=0 \mathrm{~dB}$, while has higher values at other IBO values. Moreover, the BER for all representations at the considered IBO levels are computed based on the developed BER performance in (3.34), which is a function in the measured signal parameters $R_{E}$ and $\sigma_{c}^{2}$ and given by

$$
\mathrm{BER}=Q\left(\frac{1}{2} \sqrt{\frac{\left(2-R_{E}-\sigma_{c}^{2} / \sigma_{s}^{2}\right)^{2}}{\left(\mathrm{SNR}_{\mathrm{AWGN}}\right)^{-1}+\frac{K}{L}\left(\sigma_{c}^{2} / \sigma_{s}^{2}-\frac{1}{4}\left(R_{E}+\sigma_{c}^{2} / \sigma_{s}^{2}\right)^{2}\right)}}\right)
$$

The BERs are computed at $\mathrm{SNR}_{\mathrm{AWGN}}$ of $5 \mathrm{~dB}$ and tabulated in Table 5.1, where it is evident that a representation with a minimum value of one of the nonlinearity measures of interest may achieve the best performance at certain IBO threshold values and fails at others. Therefore, it is concluded that the considered measures are not closely related to 
the system performance and do not always lead to the optimum performance. They also lack an important parameter, which is the IBO level that must be borne in mind when drafting any nonlinearity measure..

Finally, regarding the PAR issue particularly, it can be justified as follows; in the presence of a PD-HPA, the signal dynamic range is already determined by its threshold, after which the output signal is clipped (distorted). Accordingly, using the dynamic range in terms of peak power as a measure for the nonlinear distortion loses its importance, and in this case, it is better to sacrifice high peaks by letting them be clipped in favor of keeping larger portions of the signal in the linear region below the PD-HPA threshold.

\subsection{Characterization of nonlinear distortion via nonlinearity severity measure (NLS)}

In Chapter 3 and Chapter 4, we explored which signal characteristics at the HPA input are responsible for performance degradation (BER degradation and spectral regrowth). Based on such characteristics, we introduce an efficient measure to quantify the severity of the nonlinear distortion, as an alternative to the existing measures, namely nonlinearity severity (NLS) measure.

In Chapter 3, it is established that the signal characteristics contributing to BER degradation are the threshold exceeding rate $R_{E}$ and the variance of the clipped signal 
portion $\sigma_{c}^{2}$. In Chapter 4 , it is established that the signal characteristics contributing to spectral regrowth are the threshold crossing rate $R_{C}$, the variance of the clipped signal portion $\sigma_{c}^{2}$, and the mean duration of crossing event $\bar{\tau}$. For the sake of having a simplified measure, it is useful to use the interesting relation between $R_{C}$ and $\bar{\tau}$, where their product gives $R_{E}[63,64]$. Recalling the definitions of $\left.R_{C}\right|_{\text {real }}$ and $\left.R_{C}\right|_{\text {complex }}$ in (4.31) and (4.44), respectively, and the definitions of $\left.\bar{\tau}\right|_{\text {real }}$ and $\left.\bar{\tau}\right|_{\text {complex }}$ in (4.47) and (4.48), respectively, it can be shown that

$$
\begin{aligned}
\left.\left.R_{C}\right|_{\text {real }} \cdot \bar{\tau}\right|_{\text {real }} & =\frac{1}{\pi \sqrt{12}} B \exp \left(-\frac{\gamma}{2}\right) \cdot \frac{\pi \sqrt{12}}{B} \exp \left(\frac{\gamma}{2}\right) \cdot \operatorname{erfc}\left(\sqrt{\frac{\gamma}{2}}\right) \\
& =\operatorname{erfc}\left(\sqrt{\frac{\gamma}{2}}\right)=\left.R_{E}\right|_{\text {real }}
\end{aligned}
$$

and

$$
\begin{aligned}
\left.\left.R_{C}\right|_{\text {complex }} \cdot \bar{\tau}\right|_{\text {complex }} & =\sqrt{\frac{\gamma}{3 \pi}} B \exp (-\gamma) \cdot \frac{1}{B} \sqrt{\frac{3 \pi}{\gamma}} \\
& =\exp (-\gamma)=\left.R_{E}\right|_{\text {complex }}
\end{aligned}
$$

Thus, it can be argued that the signal characteristics affecting system performance, in relation to the nonlinear distortion, are limited to $R_{E}$ and $\sigma_{c}^{2}$. Thus, we define the NLS measure in a manner with similarities to the threshold crossing intensity parameter of 
[74], but explicitly using the signal characteristics that are directly linked to BER performance and spectral regrowth as

$$
\operatorname{NLS}(s(t) ; \gamma)=\frac{R_{E}(\gamma) \sigma_{c}^{2}(\gamma)}{\sigma_{s}^{2}}
$$

where a mathematical expression for NLS can be developed under the assumption that the signal (CDMA or OFDM) is characterized as a zero-mean Gaussian process as

$$
\left.\mathrm{NLS}\right|_{\text {real }}(s(t) ; \gamma)=\operatorname{erfc}\left(\sqrt{\frac{\gamma}{2}}\right)\left((1+\gamma) \operatorname{erfc}\left(\sqrt{\frac{\gamma}{2}}\right)-\sqrt{\frac{2 \gamma}{\pi}} \exp \left(-\frac{\gamma}{2}\right)\right)
$$

and

$$
\left.\mathrm{NLS}\right|_{\text {complex }}(s(t) ; \gamma)=\exp (-2 \gamma)-\sqrt{\pi \gamma} \exp (-\gamma) \operatorname{erfc}(\sqrt{\gamma})
$$

The above expressions for NLS can be verified through simulation of two example 64-user CDMA signals. The signals are filtered using an SRRC filter with rolloff of 0.22 and oversampled by 4 . The data symbols for the two signals are $a_{n}^{(k)}= \pm 1$ as representative for real signals and $a_{n}^{(k)}=( \pm 1 \pm j) / \sqrt{2}$ as representative for complex signals. Then, NLS is measured for both signals, at different values of IBO $\gamma$. The measured results are compared against the computed results using the analytic expressions in (5.7), and (5.8). Fig. 5.2 shows the curves of NLS, where it is evident that 
good agreement exists between the analytic curves and the measured points for all IBO levels.

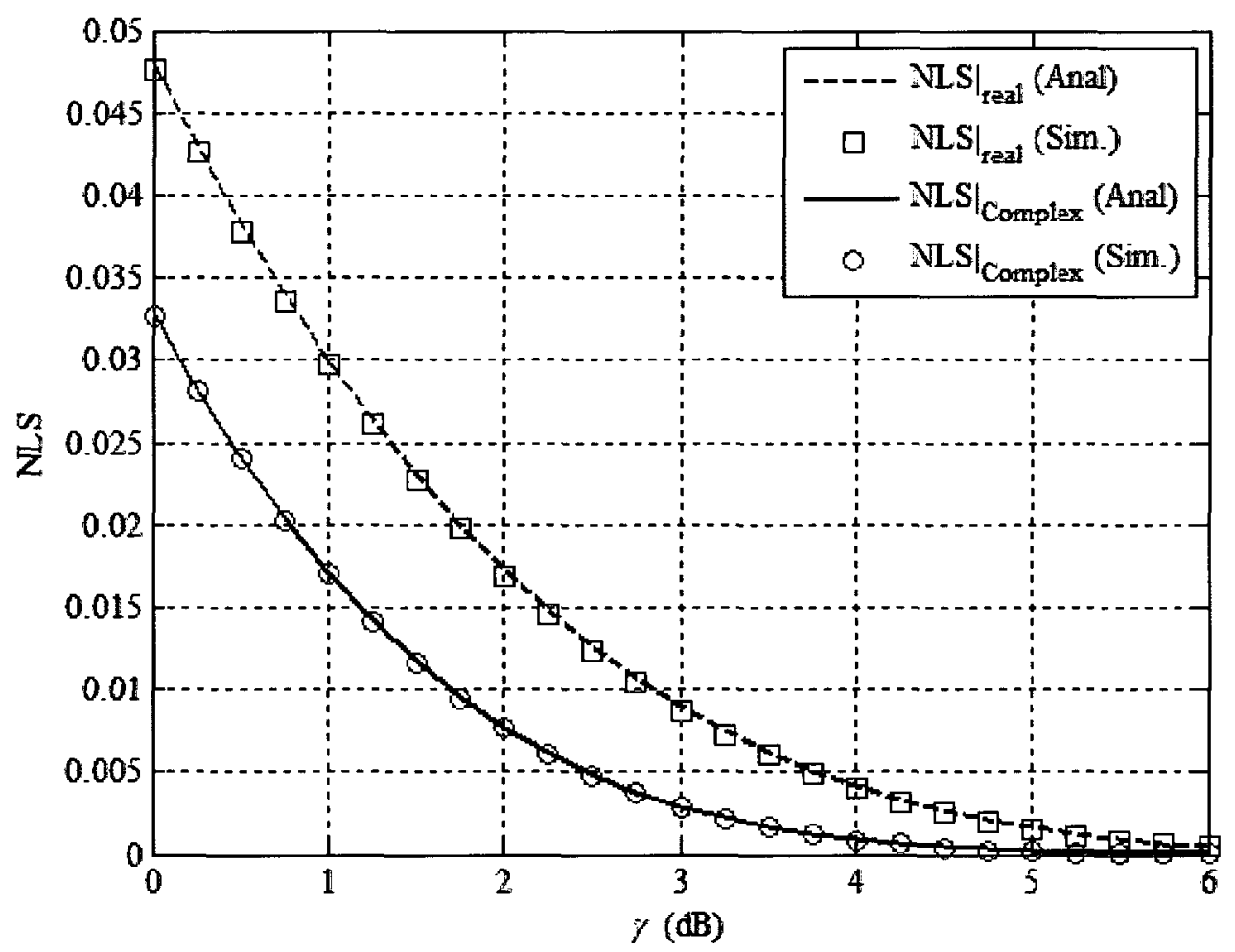

Fig. 5.2 Analytic and simulated plots of NLS versus IBO levels.

In order to emphasize the relation of the NLS measure and the signal characteristics contributing to BER degradation, recall the example drawn in Section 5.1 and adding four other representations R4, R5, R6 and R7, which are selected such that they have a minimum NLS at IBO $=0 \mathrm{~dB}, 1 \mathrm{~dB}, 2 \mathrm{~dB}$, and $3 \mathrm{~dB}$, respectively. The NLS measure is measured for all representations and shown along with the other parameters of interest in Table 5.2. 
Table 5.2 Signal characteristics of representations R1, R2, R3, R4, R5, R6, and R7.

\begin{tabular}{|c|c|c|c|c|c|c|c|c|c|}
\hline & $\mathbf{R 1}$ & $\mathbf{R 2}$ & R3 & R4 & R5 & R6 & $\mathbf{R} 7$ & & \\
\hline $\begin{array}{l}\text { PAR } \\
\text { (dB) }\end{array}$ & 4.46 & 5.73 & 8.85 & 5.59 & 5.52 & 5.28 & 5.37 & & \\
\hline IPV & 0.61 & 0.51 & 1.51 & 0.56 & 0.59 & 0.55 & 0.51 & & \\
\hline $\mathrm{CM}$ & 0.36 & 0.36 & 0.12 & 0.36 & 0.34 & 0.29 & 0.32 & & \\
\hline \multirow{4}{*}{$R_{E}$} & 0.432 & 0.489 & 0.337 & 0.323 & 0.451 & 0.447 & 0.496 & 0 & \multirow{12}{*}{$\begin{array}{l}\widehat{\theta} \\
\frac{0}{2}\end{array}$} \\
\hline & 0.280 & 0.341 & 0.322 & 0.281 & 0.285 & 0.360 & 0.386 & 1 & \\
\hline & 0.246 & 0.164 & 0.212 & 0.193 & 0.189 & 0.170 & 0.193 & 2 & \\
\hline & 0.140 & 0.083 & 0.155 & 0.076 & 0.095 & 0.061 & 0.057 & 3 & \\
\hline \multirow{4}{*}{$\sigma_{c}^{2}$} & 0.058 & 0.125 & 0.048 & 0.050 & 0.058 & 0.052 & 0.047 & 0 & \\
\hline & 0.027 & 0.033 & 0.142 & 0.034 & 0.024 & 0.028 & 0.035 & 1 & \\
\hline & 0.015 & 0.015 & 0.097 & 0.022 & 0.018 & 0.011 & 0.014 & 2 & \\
\hline & 0.006 & 0.004 & 0.063 & 0.010 & 0.008 & 0.006 & 0.004 & 3 & \\
\hline \multirow{4}{*}{$\begin{array}{l}\text { NLS } \\
(\mathrm{dB})\end{array}$} & -15.98 & -12.15 & -17.90 & -17.92 & -15.85 & -16.35 & -16.30 & 0 & \\
\hline & -21.24 & -19.43 & -13.41 & -20.39 & -21.59 & -19.89 & -18.67 & 1 & \\
\hline & -24.23 & -26.16 & -16.87 & -23.81 & -24.64 & -27.51 & -25.83 & 2 & \\
\hline & -30.47 & -35.07 & -20.08 & -31.18 & -31.29 & -34.55 & -36.52 & 3 & \\
\hline
\end{tabular}


As can be seen in Table 5.2, the values of $R_{E}$ and $\sigma_{c}^{2}$ measured for the representations with the minimum NLS adjusted to the considered IBO levels are very close to the minimum values of $R_{E}$ and $\sigma_{c}^{2}$ measured for the other representation at the corresponding IBO level. Also, the BER for all representations at all IBO levels are computed at $\mathrm{SNR}_{\mathrm{AWGN}}$ of $5 \mathrm{~dB}$ and shown in Fig. 5.3 , where it is clear that the representations with minimum NLS adjusted to a particular IBO level lead to the best BER performance at that level.

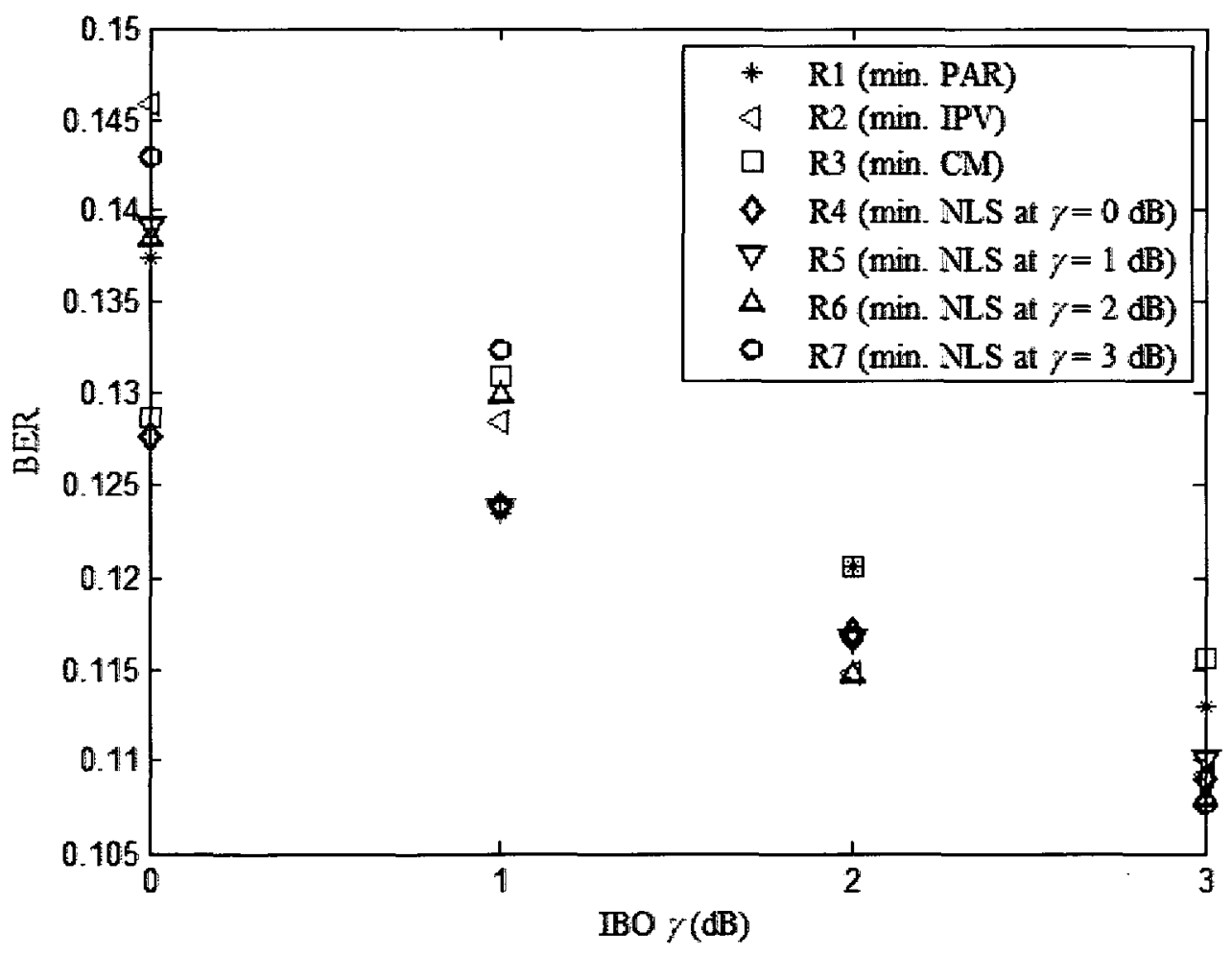

Fig. 5.3 BER of (3.34) computed for R1-R7 versus IBO levels. 
It appears evident here is another advantage of the NLS measure, in addition to being directly linked to the BER performance and spectral regrowth, which is the correlation between the NLS measure and the IBO level.

\subsection{Conclusions}

In this chapter, we presented a brief review of the existing measures used to characterize the nonlinear distortion showing that such measures are not mathematically related to the system performance and do not always lead to optimum performance. Based on the signal characteristics developed in Chapter 3 and Chapter 4 that represent the main contributors to BER degradation and spectral regrowth, a new measure, NLS, which better characterizes the nonlinear distortion, is developed. This parameter is characterized by having a direct link to the system performance. Also, being a function in the IBO level gives the NLS measure a superior advantage as it can be adjusted to the IBO level required to optimize performance according to design demands, which is not possible with the existing measures. Additionally, this measure can be used as a selection criterion in search and optimization techniques, like SLM and PTS, to select the best signal representation to be transmitted. 


\section{Chapter 6}

\section{PERFORMANCE ASSESSMENT}

\section{OF THE NONLINEARITY SEVERITY MEASURE}

I $\mathrm{n}$ this chapter, the performance of the NLS is assessed. Two performance merits are used in the assessment: BER and PSD as a measure for spectral regrowth. The selected mapping (SLM) technique is adopted as a platform for performance assessment since it represents one of the well known search and optimization techniques that lead to improved performance in both CDMA and OFDM. In SLM, several representations of the signal to be transmitted are generated, where there should be a selection criterion, upon which the best representation is selected for transmission.

Although any of the nonlinearity measures (PAR, IPV, and CM) described in Chapter 5 can be used as a selection criterion in SLM, PAR is the most commonly used one. This may be because of its simpler form and the numerous investigations and discussions conducted on it in the literature. Accordingly, in this dissertation, the performance of the NLS is assessed in comparison with PAR as selection criteria in SLM. 
Then, the performance of the NLS measure is examined with different HPA models ${ }^{1}$ to show that it can also lead to an improved performance. In these experiments, Saleh's model and Rapp's model are used as examples for TWTAs and SSPAs, respectively.

\subsection{Selected mapping (SLM) technique}

Various techniques have been proposed in the literature to reduce the effects of amplifier nonlinearities on CDMA and OFDM signals, where the PAR is often used as the nonlinearity measure. Clipping of large signal peaks is the most straightforward technique $[8-10,75-78]$. However, this technique results in performance degradation in terms of BER degradation and spectral regrowth since it distorts the input signal to the amplifier $[89,80]$. Companding techniques have been proposed for OFDM systems [1113, 81-85]. Companding achieves improvement in the BER; however, expansion of the compressed signal at the receiver yields noise amplification which can counteract any of the performance gains that would otherwise accrue from the increased SNR at the receiver [86-89]. Also, search and optimization techniques such as PTS and SLM have been introduced and implemented to select the best signal for transmission and, in turn, improve the system performance. Such techniques usually adopt the minimum PAR as the selection criterion. In PTS [17-19, 90-93], the signal is partitioned into multiple

\footnotetext{
${ }^{1}$ no predistorter is involved in the amplifier chain
} 
disjoint subblocks, where the lowest PAR signal is selected by optimally phase combining the signal subblocks.

In this dissertation, we use the SLM technique as the platform for assessing the performance of the NLS measure, which was originally proposed for OFDM and then applied to CDMA [14-16, 47, 94-100]. In the following subsections, we present the basics of the SLM technique used in both the CDMA and OFDM systems.

\subsubsection{SLM in CDMA}

The concept behind the existing SLM technique is based on creating $Q$ equivalent representations of the same signal $s(t)$ by rotating the phases of the symbols. Then, the signal representation that has the minimum PAR is selected for transmission. The block diagram of the SLM technique in a typical CDMA system is depicted in Fig. 6.1, where the branches $k=1, \ldots, K$ represent the number of users.

A phase rotation vector $\mathbb{P}=\left[P_{1}, \ldots, P_{M}\right]$ with $P_{m}=e^{j \varphi_{m}}, \varphi_{m}=2 \pi m / M$, $m=1, \ldots, M$ is defined. Each $k$ th user's data symbol $a^{(k)}$ is element-wise multiplied by the vector $\mathbb{P}$, resulting in a set of $M$ different symbols. These $M$ symbols are then multiplied by the corresponding spreading waveform $c^{(k)}(t)$ forming $M$ different representations for each user as

$$
s_{k}^{(m)}(t)=a^{(k)} P_{m} c^{(k)}(t), \quad 0 \leq t \leq T
$$




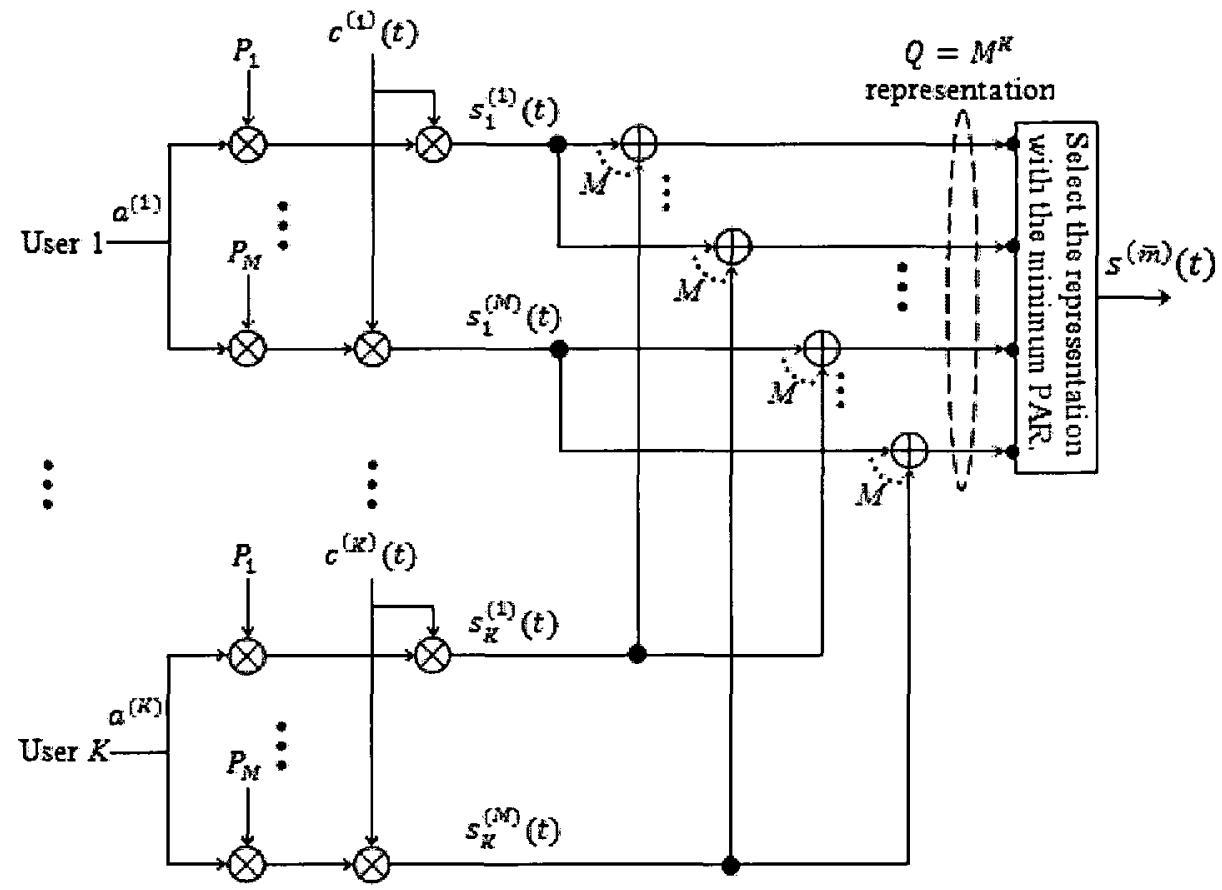

Fig. 6.1 Block diagram of the SLM technique for a CDMA system.

Consequently, $Q=M^{K}$ signal representations are generated by performing all possible combinations of the $M$ representations for each $k$ th user signal such that $s(t) \in\left\{s^{(q)}(t)\right\}_{q=1}^{Q}$. Among the $Q$ signal representations, the representation $\bar{m}$ that has the minimum PAR is selected for transmission as follows

$$
\begin{aligned}
\bar{m} & =\arg \min _{1 \leq q \leq Q} \psi^{(q)} \\
& =\arg \min _{1 \leq q \leq Q} \frac{\max _{0 \leq t \leq T}\left|s^{(q)}(t)\right|^{2}}{\sigma_{s}^{2}} .
\end{aligned}
$$


The SLM technique remarkably improves the system performance, but at the expense of an increased system complexity and computational burden. The system in this case performs a large number of computations since the number of the generated representations grows exponentially with the number of users $\left(Q=M^{K}\right.$ representations). For instance, for an 8-user CDMA system with a phase rotation vector $\mathbb{P}$ consisting of 4 elements, the total number of possible representations is 65536 . In this case, the system suffers a tremendous computational burden through performing an increased number of multiplications, in addition to the search and optimization procedures that follow. The complexity reduction issue in the SLM technique was often raised in the literature and various proposals have been presented [94-97, 100]. For the sake of reducing the number of generated representations, we showed in [101] that the optimization procedure can be performed in a greedy manner. Adopting a greedy algorithm in the search and optimization procedure yields a suboptimal selection of the signal to be transmitted. A greedy algorithm is an algorithm that always takes the best immediate, or local, solution while finding an answer at each stage with the hope of finding the global optimum [102]. Using such a greedy algorithm strongly reduces the number of representations among which the system selects the optimum one to be transmitted to $Q=K(M+1)-M$. Clearly, by applying the greedy algorithm, the number of the generated representations grows linearly with the number of users. 
A complexity reduction ratio (CRR) can be defined in a manner with similarities to that of [103], but using the number of representations generated in each of the optimal and greedy procedures as

$$
\operatorname{CRR}(\%)=\left(1-\frac{\left.Q\right|_{G R D}}{\left.Q\right|_{O P T}}\right) \times 100 \%
$$

Table 6.1 CRR for the optimal and greedy algorithms.

\begin{tabular}{|c|c|c|c|c|}
\hline$K$ & $M$ & $\left.Q\right|_{O P T}$ & $\left.Q\right|_{G R D}$ & CRR (\%) \\
\hline \multirow{3}{*}{2} & 2 & 4 & 4 & 0 \\
\cline { 2 - 5 } & 4 & 16 & 6 & 62.5 \\
\cline { 2 - 5 } & 8 & 64 & 10 & 84.4 \\
\hline \multirow{3}{*}{4} & 2 & 16 & 10 & 37.5 \\
\cline { 2 - 5 } & 4 & 256 & 16 & 93.8 \\
\cline { 2 - 5 } & 8 & 4096 & 28 & 99.3 \\
\hline \multirow{3}{*}{8} & 2 & 256 & 22 & 91.4 \\
\cline { 2 - 5 } & 4 & 65536 & 36 & 99.94 \\
\cline { 2 - 5 } & 8 & 16777216 & 64 & 99.99 \\
\hline
\end{tabular}

Table 6.1 shows the number of representations generated using both the optimal full search procedure $\left.Q\right|_{O P T}$ and the greedy algorithm $\left.Q\right|_{G R D}$ for different number of users $K$ and different number of representations generated for each user in the system $M$. 
The CRR for both procedures is shown as well, where it is evident that a remarkable improvement in complexity reduction in terms of reduced number of representations exists.

The issue of computational complexity has been extensively discussed and many approaches have been proposed to reduce the complexity for multiuser systems [96-101]. It was shown that the multiplication by the phase rotation vector to obtain different representations of the same signal represents the main contributor to the system complexity, especially in multicarrier and OFDM systems, where complexity becomes even more severe since they require several IFFT operations to generate different signal representations. For the purpose of reducing such computational burden, we proposed in [101] a low complexity technique, namely cyclically shifted sequences (CSS). In the CSS technique, the multiplication operations are replaced by cyclic shifting of the users' data sequences, which greatly reduces the system complexity. Consider the signal block to be transmitted consisting of $N$ symbols, where the $k$ th user signal has the form

$$
s_{k}(t)=\sum_{n=0}^{N-1} a_{n}^{(k)} c^{(k)}(t-n T)
$$

The CSS technique is based on creating $(M+1)$ representations for every user's data sequence $\left\{a_{n}^{(k)}\right\}_{n=0}^{N-1}$ by applying $M$ cyclic shifts to the symbols of that sequence. Hence, the $m$ th representation of the $k$ th user signal can be expressed as 


$$
s_{k}^{\left(m_{k}\right)}(t)=\sqrt{E_{k}} \sum_{n=0}^{N-1} a_{\left(\left(n-m_{k}\right)\right)_{N}}^{(k)} c^{(k)}(t-n T),
$$

where $m_{k}=0, \ldots, M$ denotes the $m$ th shift assigned to the $k$ th user data sequence and $((\cdot))_{N}$ is the modulo- $N$ operator.

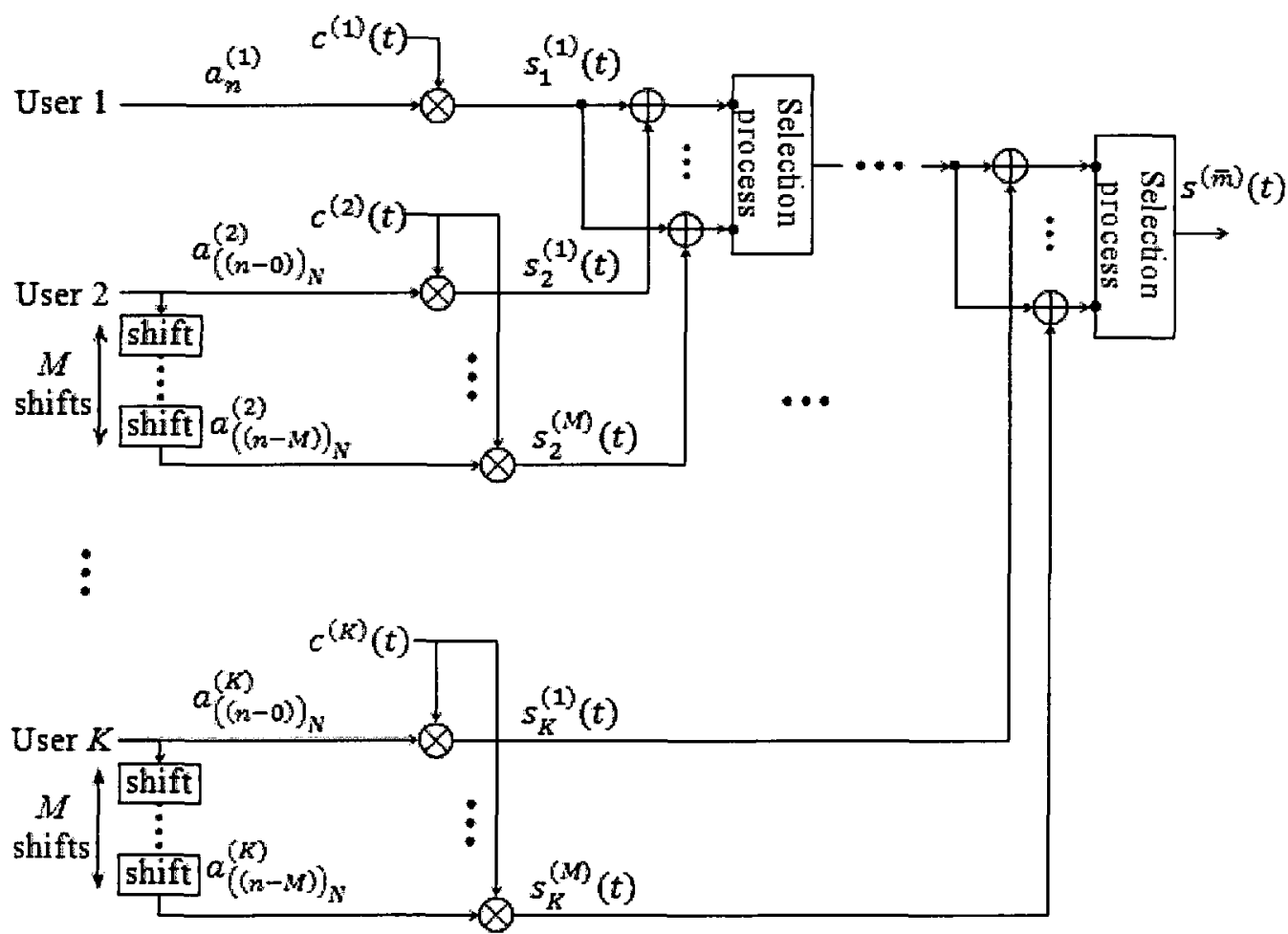

Fig. 6.2 Block diagram of the CSS technique for one subblock.

In essence, we have 


$$
\begin{aligned}
s^{(q)}(t) & =\sum_{k=1}^{K} s_{k}^{\left(m_{k}\right)}(t) \\
& =\sum_{k=1}^{K} \sqrt{E_{k}} \sum_{n=0}^{N-1} a_{\left(\left(n-m_{k}\right)\right)_{N}}^{(k)} c^{(k)}(t-n T),
\end{aligned}
$$

where $q \in\{1, \ldots, Q\}$ and $Q$ denotes the total number of signal representations according to the number of shifts assigned to each user data sequence. Among the $Q$ signal representations $\left\{s^{(q)}(t)\right\}_{q=1}^{Q}$, the best representation $\bar{m}$ is selected for transmission. The CSS technique using a greedy algorithm for one block is depicted in Fig. 6.2.

In order to assess the performance of the CSS technique in comparison with the SLM technique, four different representations of an 8-user CDMA signal with Walsh codes of length $L=64$ and SRRC filtered are generated using: 1) SLM with optimal algorithm, 2) SLM with greedy algorithm, 3) CSS with optimal algorithm, and 4) CSS with greedy algorithm, with $M=4$ for the number of elements in the phase rotation vector and the number of shifts in the SLM and CSS, respectively. For each representation, the complementary cumulative distribution function (CCDF) of the instantaneous power normalized to the average power is computed and plotted in Fig. 6.3. It is clear from the figure that the PAR reduction achieved by the conventional SLM technique and the proposed CSS technique are similar. Also, it can be seen that adopting a greedy algorithm shows a close performance to that of the optimal algorithm up to an 
IBO of $4 \mathrm{~dB}$, which is quite enough to linearly pass most of the signal in presence of a PD-HPA in the cases of fewer users $(K \leq 12)$.

It is worth mentioning that the $k$ th receiver must know the number of shifts $m_{k}$ in order to undo the shifting process for the received data sequence, and hence, the number of shifts should be transmitted as side information. Transmission of the side information is beyond the scope of this dissertation. However, there are various ways to manage the side information issue, one of which is transmitting the side information about $m_{k}$ over an overhead channel. Also, a few publications have addressed that issue and proposed different ways of transmission without (or with embedded) side information $[18,100$, $104]$.

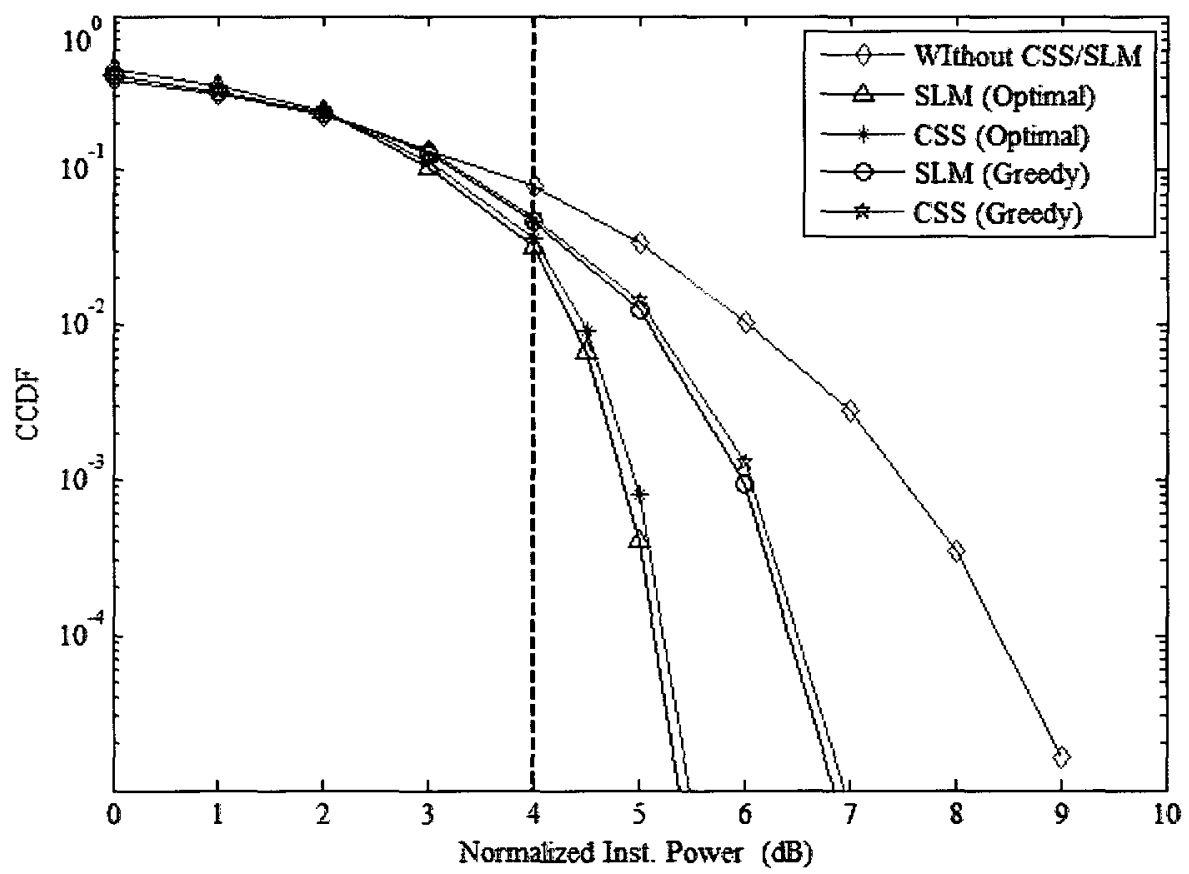

Fig. 6.3 CCDFs of the normalized instantaneous power of an 8-user DS-CDMA signal. 


\subsubsection{SLM in OFDM}

SLM was first introduced for OFDM systems by Bauml et al. [14]. In OFDM transmission schemes, a block of $N$ distinct complex-valued subcarriers (OFDM frame) is transformed into the time-domain using IFFT. The phase of each data block is rotated before the IFFT by $M$ phase vectors resulting in $M$ equivalent distortionless representations. The one with the lowest PAR of these representations is retained and transmitted instead of the original OFDM signal. The complex baseband OFDM signal can be represented as

$$
s(t)=\frac{1}{N} \sum_{k=1}^{N} A_{k} e^{j 2 \pi f_{k} t}, \quad 0 \leq t \leq T
$$

where $A_{k}$ is the data symbol carried by the $k$ th subcarrier, $T$ is the OFDM symbol duration, and $N$ is the number of subcarriers. The OFDM signal can be expressed in discrete time by sampling $s(t)$ at a sampling rate of $1 / T$ as

$$
\left.s_{n} \triangleq s(t)\right|_{t=\frac{n T}{N}}=\frac{1}{N} \sum_{k=1}^{N} A_{k} e^{\frac{j 2 \pi n k}{N}}, \quad n=1, \ldots, N
$$

Let the data sequence in the OFDM block be expressed as an $N$-dimensional vector $\mathbb{A}=\left[A_{1}, A_{2}, \ldots, A_{N}\right]^{T}$ and define the $m$ th phase rotation vector as $\mathbb{P}_{m}=$ $\left[P_{1}^{(m)}, P_{2}^{(m)}, \ldots, P_{N}^{(m)}\right]^{T}$, where $P_{k}^{(m)}=e^{j \varphi_{k}^{(m)}}$ with $\varphi_{k}^{(m)}=2 \pi m / M, m=1, \ldots, M$. Then, 
the frequency-domain version $\mathbb{S}_{m}$ of the $m$ th candidate signal can be generated by performing array multiplication (carrier-wise multiplication) of the data vector $\mathbb{A}$ and $m$ th phase rotation vector $\mathbb{P}_{m}$ as follows

$$
\mathbb{S}_{m}=\left[P_{1}^{(m)} A_{1}, P_{2}^{(m)} A_{2}, \ldots, P_{N}^{(m)} A_{N}\right]^{T}
$$

A set of transmission candidate signals $\mathbb{s}_{m}$ can be generated by performing the IFFTs of the frequency-domain vectors $\mathbb{S}_{m}$. The candidate signal that has the lowest PAR is selected for transmission. The block diagram of the SLM technique in a typical OFDM system is depicted in Fig. 6.4.

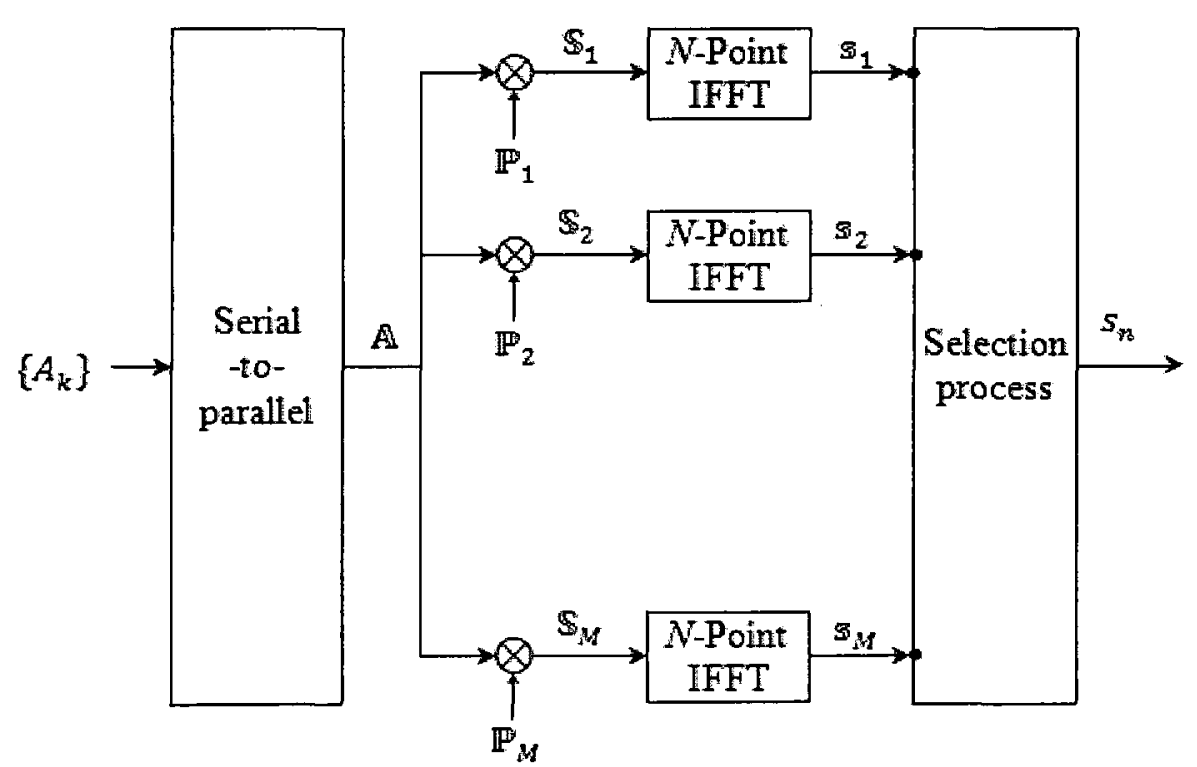

Fig. 6.4 Block diagram of the SLM technique for an OFDM system. 


\subsection{Performance assessment of NLS in presence of PD-HPA}

In our approach to assess the performance of the NLS measure using the SLM technique, the best representation $\bar{m}$ is selected for transmission among the $Q$ equivalent representations such that it has the minimal NLS at every IBO level of interest as follows

$$
\bar{m}=\arg \min _{1 \leq q \leq Q} \operatorname{NLS}(q)(s(t) ; \gamma)
$$

Performance assessment is conducted through computer simulations for both CDMA and OFDM systems. Three CDMA signals with Walsh orthogonal codes of length $L=64$ are considered with different number of users $K=32,16$, and 8 . For OFDM, three signals with block lengths (number of symbols per OFDM block) of $N=$ 128,64 , and 16 (without cyclic prefix) subjected to $N=128,64$, and 16 subcarriers, respectively, are considered. Data symbols are QPSK modulated. An SRRC pulse shaping filter is used with roll-off factor of $\rho=0.22$ and upsampling factor of 4 in order to obtain an adequate signal representation in a nonlinear environment.

The performance of the NLS measure is assessed through a comparison against PAR as two selection criteria in the SLM technique to determine the best signal to be transmitted. For such a purpose, three different representations for each of the six signals are generated as follows: 1) with no SLM optimization, 2) with SLM using the minimum PAR as the selection criterion, and 3) with SLM using the minimum NLS adjusted to the 
IBO level of interest as the selection criterion. All representations are subjected to a PDHPA amplifier chain. Two performance merits are used in the assessment: BER and $\mathrm{ACPR}$ as a measure for spectral regrowth. Numerical results are given in the following subsections.

\subsubsection{BER performance}

The BER performance results presented in this section for CDMA represent the average BER of 100 frames of each CDMA signal representation of interest. Each frame consists in 10,000 random QPSK data symbols. The average BER for OFDM is carried out over 1,000,000 OFDM blocks for each signal representation; each block is of length $N$ random QPSK data symbols. Results are obtained at three IBO levels $\gamma=1 \mathrm{~dB}, 2 \mathrm{~dB}$, and $3 \mathrm{~dB}$.

The BER performance curves for each signal representation in the presence of a PD-HPA at the considered IBO levels are plotted in Fig. 6.5-6.10. Fig. 6.5, Fig 6.6, and Fig. 6.7 show the BER curves for the 32-, 16-, and 8-user CDMA signals, respectively. While the BER curves for the 128-, 64-, and 16- OFDM signals are plotted in Fig. 6.8, Fig 6.9, and Fig. 6.10, respectively. 


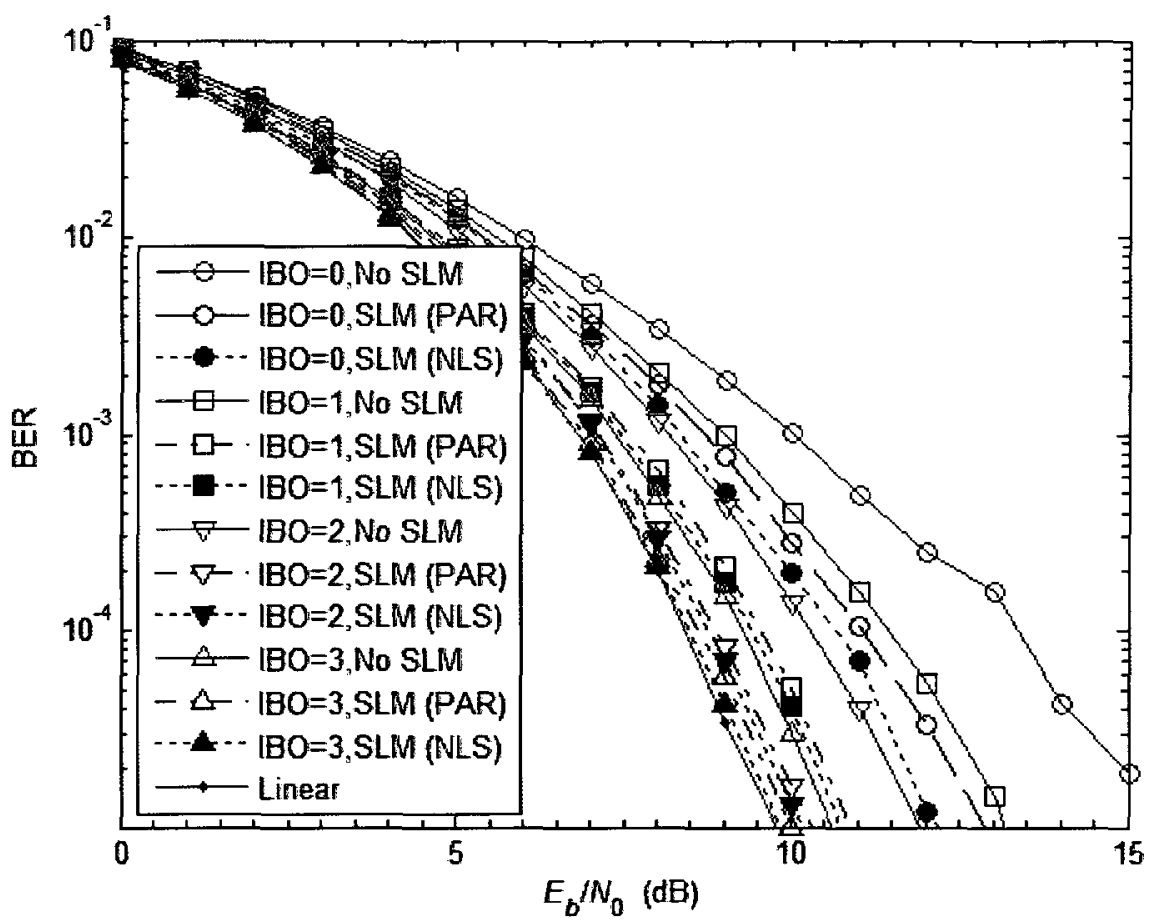

Fig. 6.5 BER of 32-user CDMA signals in presence of PD-HPA.

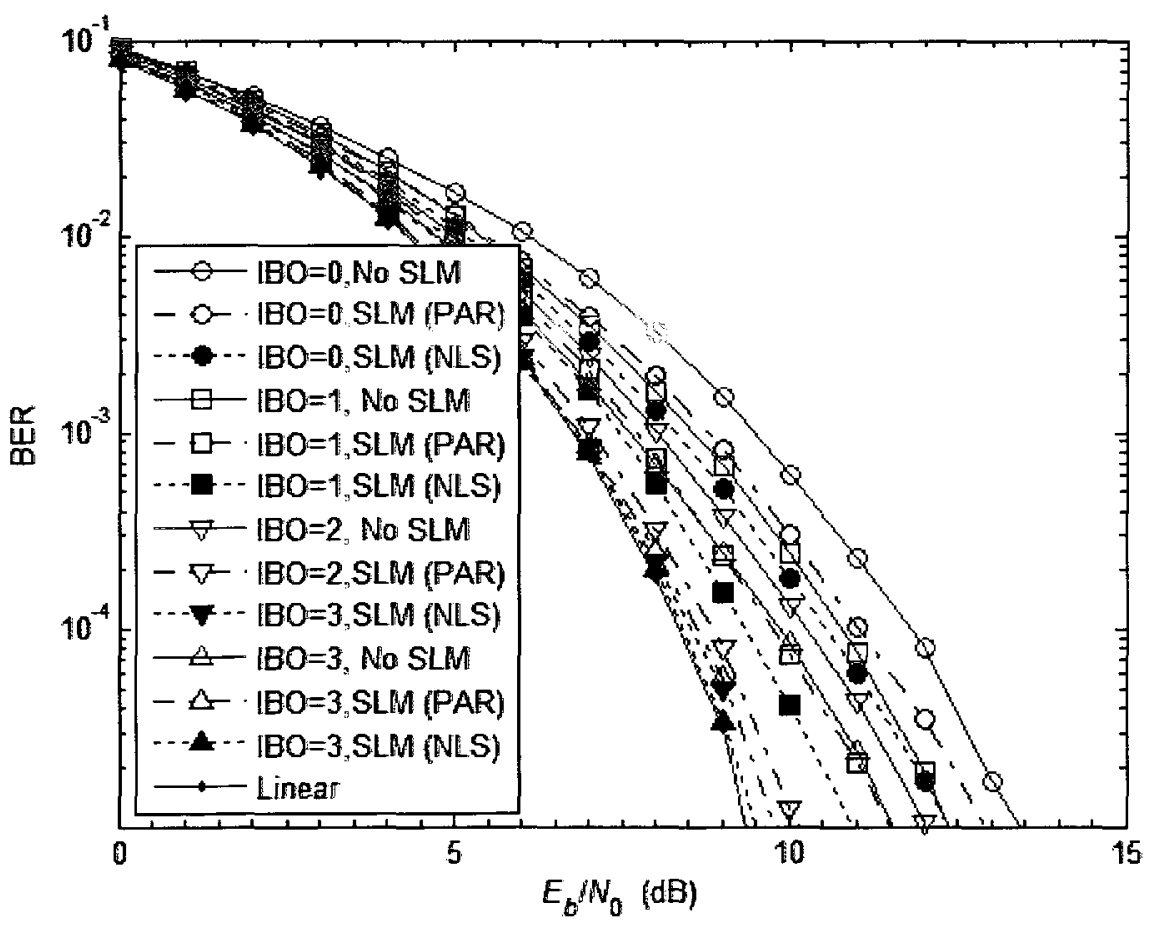

Fig. 6.6 BER of 16-user CDMA signals in presence of PD-HPA. 


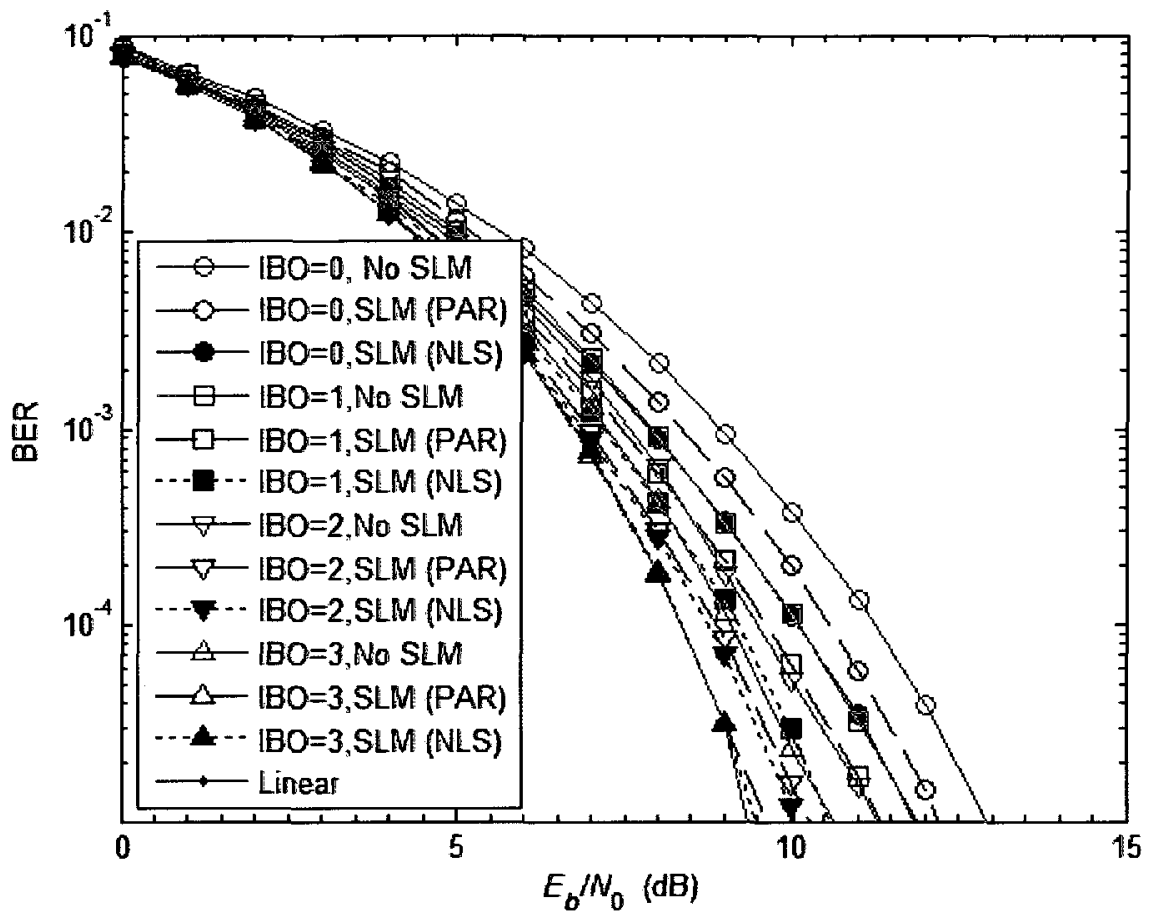

Fig. 6.7 BER of 8-user CDMA signals in presence of PD-HPA.

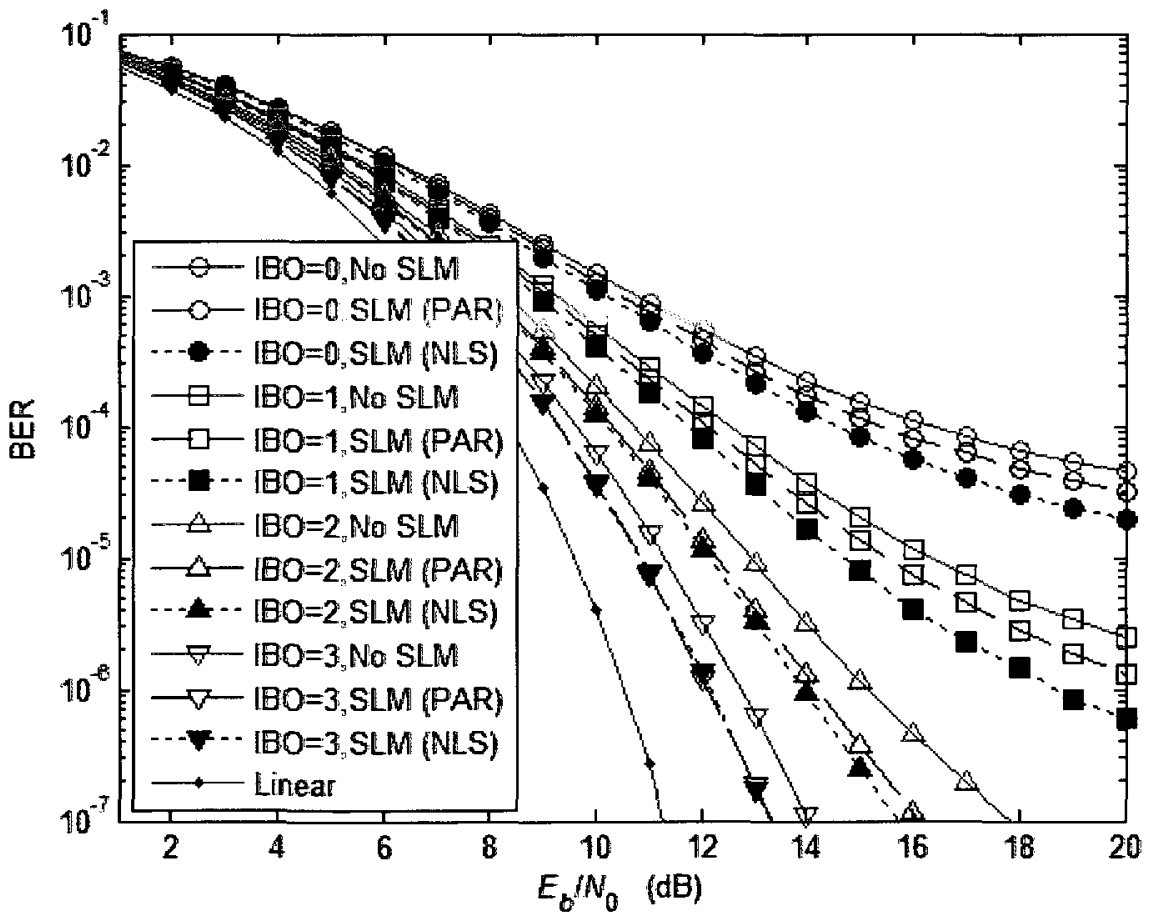

Fig. 6.8 BER of 128-OFDM signals in presence of PD-HPA. 


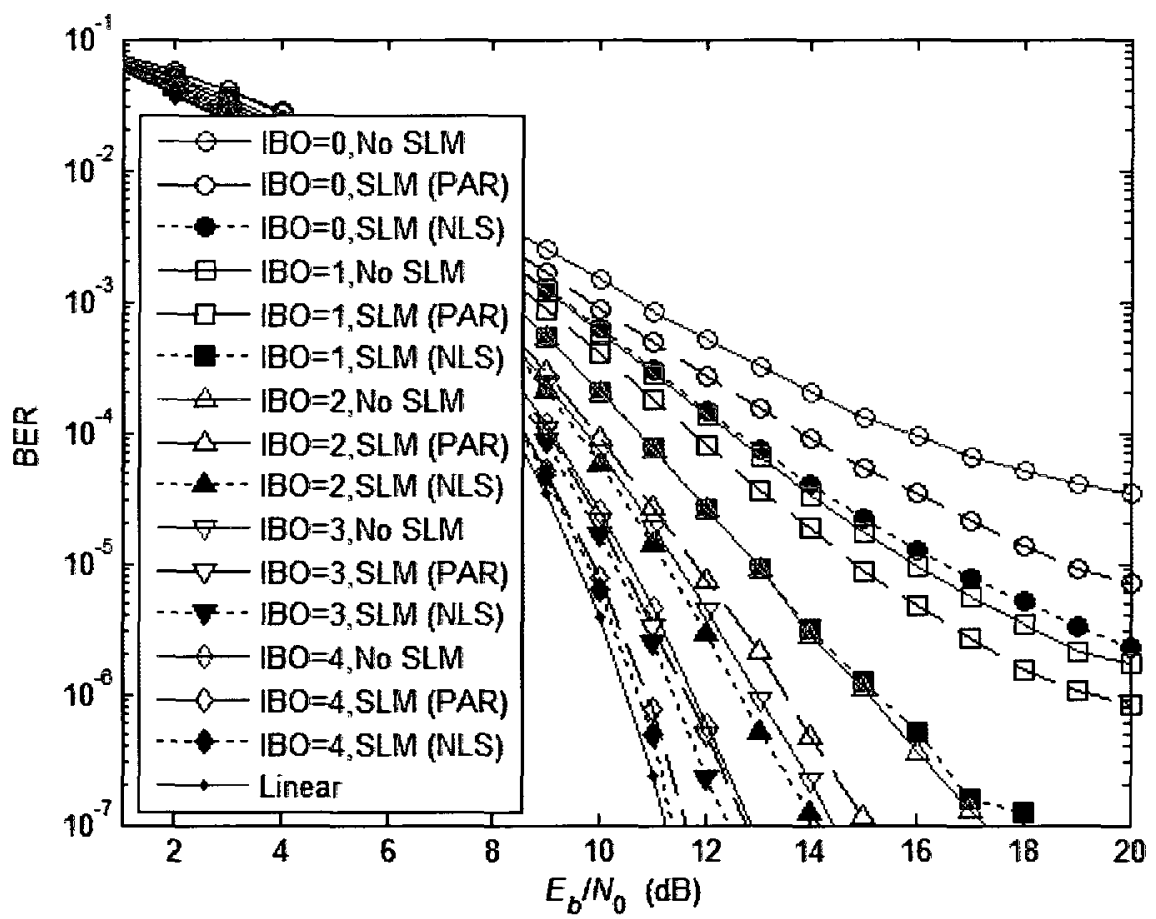

Fig. 6.9 BER of 64-OFDM signals in presence of PD-HPA.

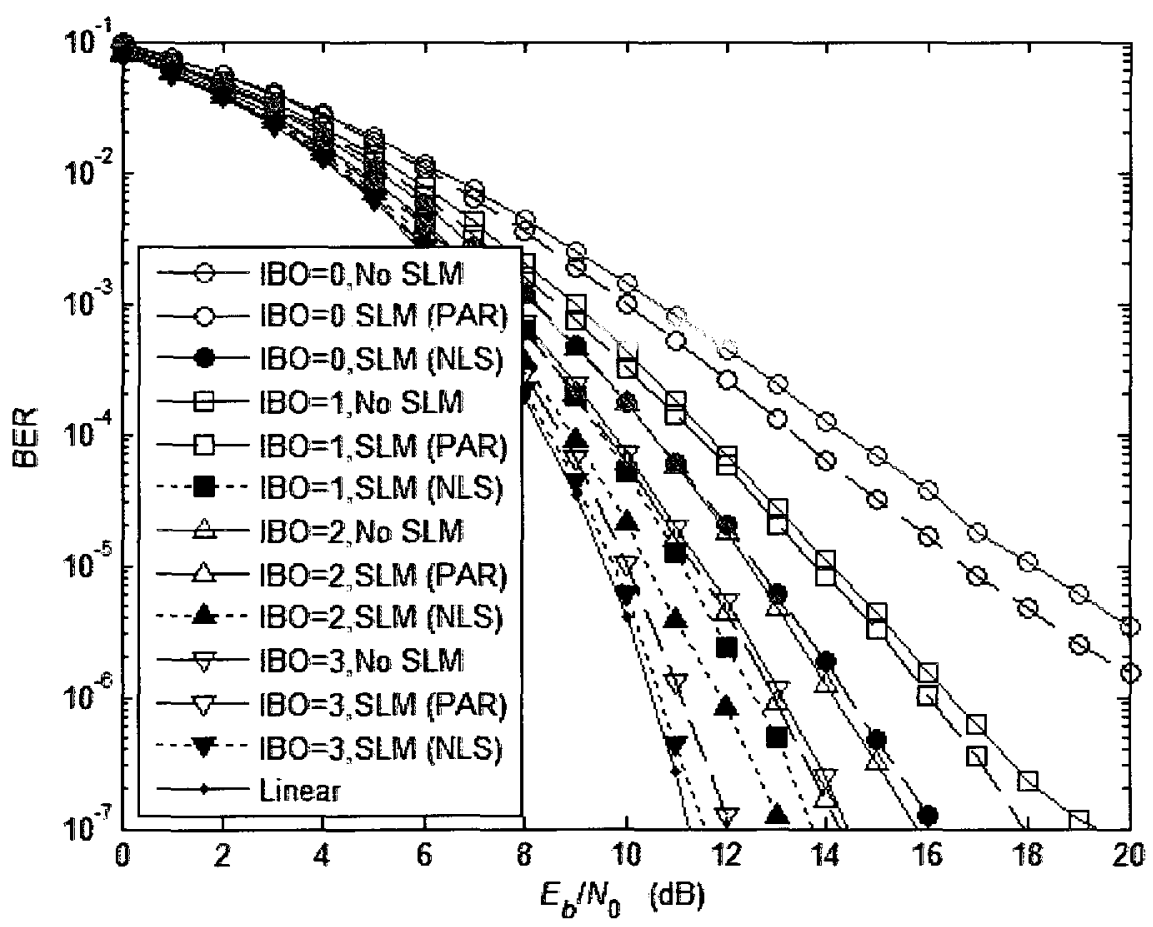

Fig. 6.10 BER of 16-OFDM signals in presence of PD-HPA. 
It is clear from Fig. $6.5-6.10$ that using the minimum NLS measure adjusted to the IBO level of interest, compared to the minimum PAR as selection criteria to select the signal to be transmitted, improves the BER performance. This was expected since the NLS measure is based on factors linked directly to the BER performance, $R_{E}$ and $\sigma_{c}^{2}$, as we showed in (3.34). Also, it is worth mentioning that a representation selected based on the minimum NLS criterion may also have the minimum PAR. In this case, both criteria will lead to the same performance.

\subsubsection{Spectral regrowth}

In this subsection, we use the upper ACPR as a measure to assess the performance of using the NLS measure as a selection criterion in the SLM technique in comparison with PAR. To compute the ACPR, the PSD of each of the three representations (no SLM optimization, SLM using NLS, and SLM using PAR) for each of the six signals under test (32-, 16-, and 8-user CDMA and 128-, 64-, and 16- OFDM) is computed. The PSDs for time domain signal representations of length $10 \mathrm{~L}$ (Walsh code length) and $10 \mathrm{~N}$ for CDMA and OFDM, respectively, are evaluated using the Welch estimation method in MATLAB $^{\circledR}$ (version R2009b) with the following parameters: Hamming window and $50 \%$ overlap between segments. In order to have an adequate trade-off between estimate reliability and frequency resolution, the segment length is set to 32 . 
The PSDs for the signal representation in the presence of a PD-HPA at three IBO levels $\gamma=1 \mathrm{~dB}, 2 \mathrm{~dB}$, and $3 \mathrm{~dB}$, are plotted in Fig. 6.11-6.16. Fig. 6.11-6.13 shows the PSDs for the 32-, 16-, and 8-user CDMA signals, respectively. While the PSDs for the 128-, 64-, and 16-channel OFDM signals are plotted in Fig. 6.14-6.16, respectively. The PSDs of the signal representations with no SLM optimization are omitted from the plots to allow for clearer comparisons between the two representations of interest in; SLM using NLS and SLM using PAR. However, the results of the computed ACPRs for all representations are tabulated in Table $6.2-6.7$. 


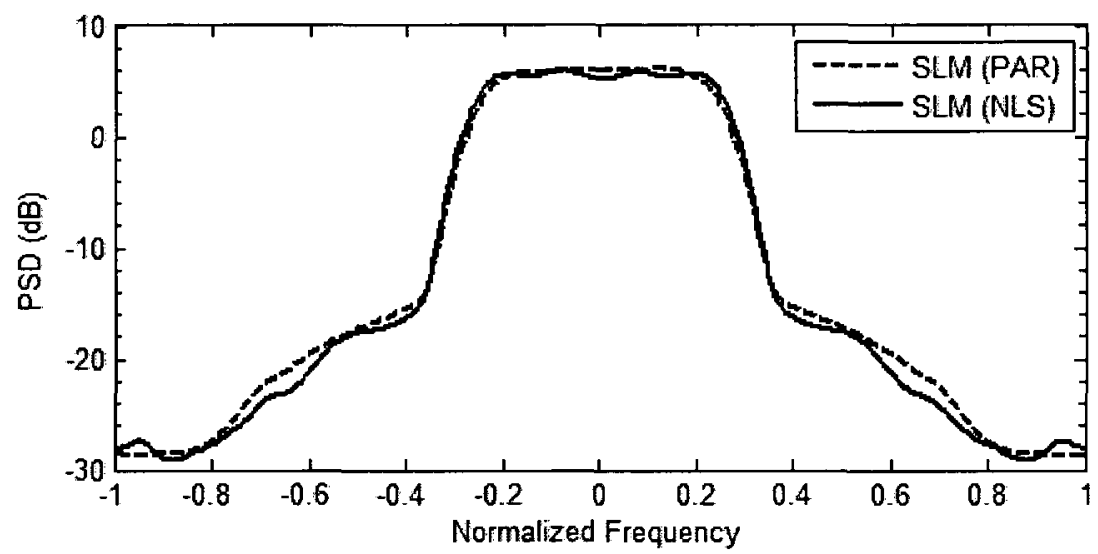

(a)

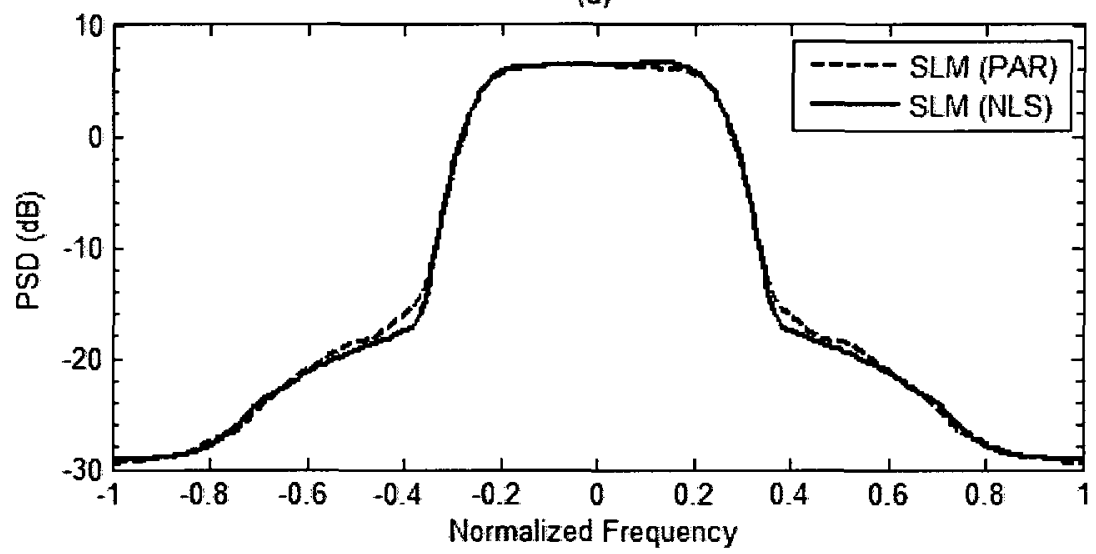

(b)

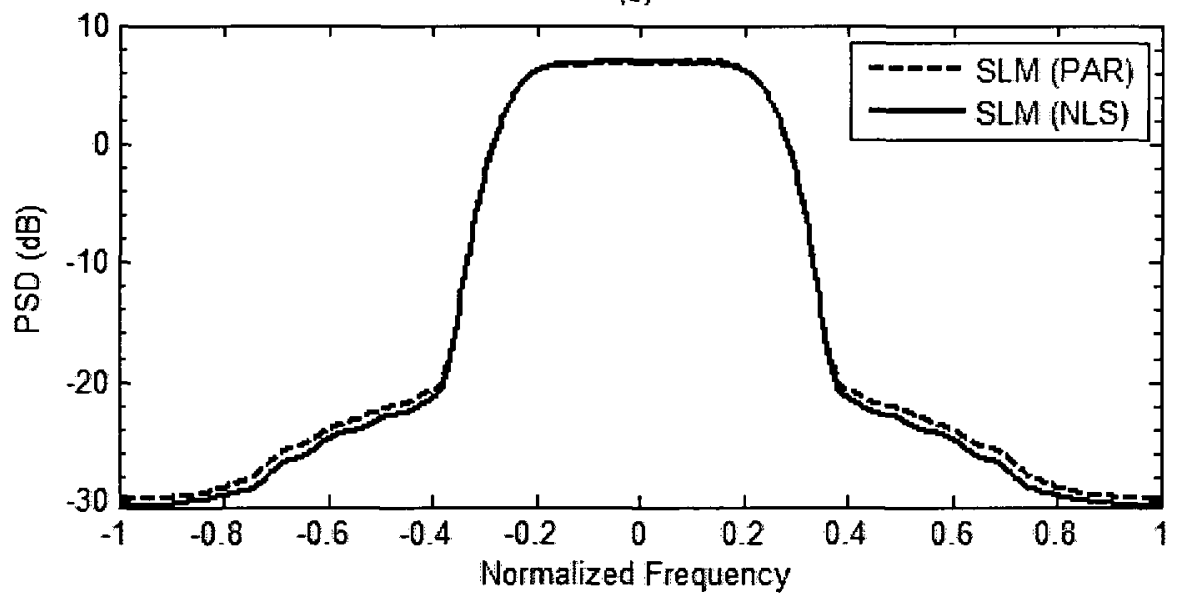

(c)

Fig. 6.11 PSDs of 32-user CDMA signals in presence of PD-HPA at (a) IBO = 1 $\mathrm{dB}$, (b) $\mathrm{IBO}=2 \mathrm{~dB}$, and (c) $\mathrm{IBO}=3 \mathrm{~dB}$. 


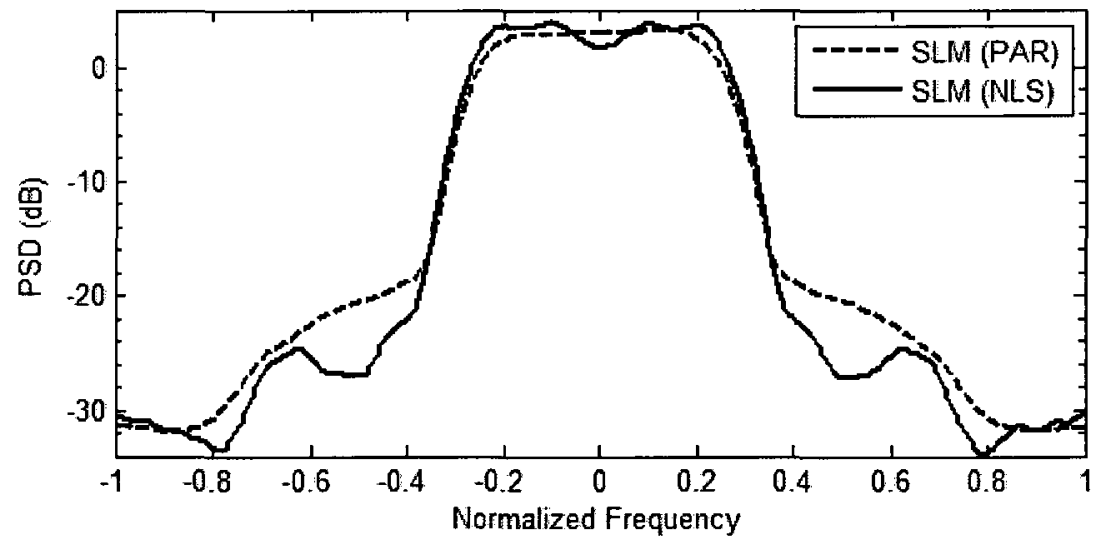

(a)

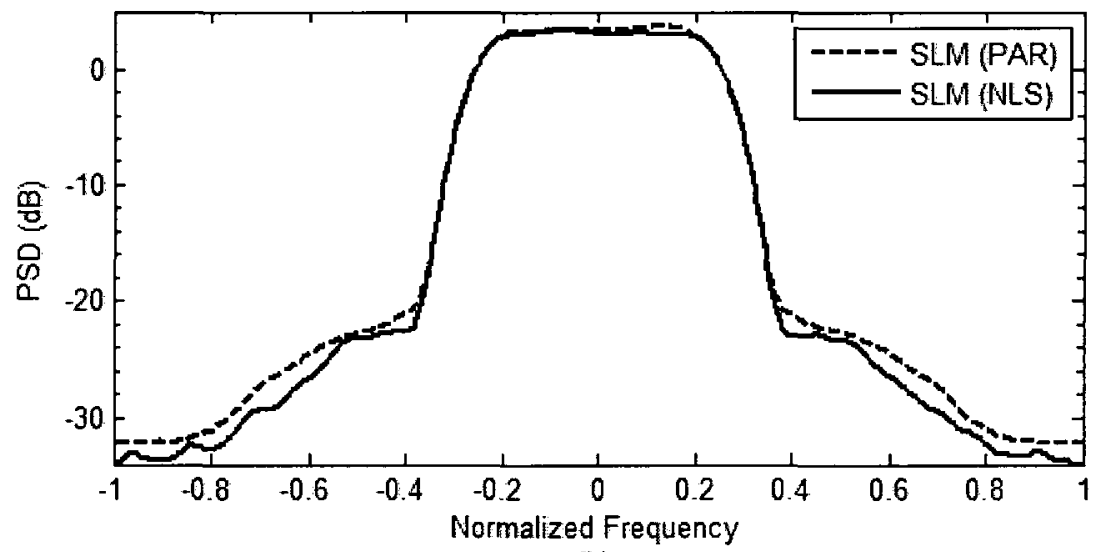

(b)

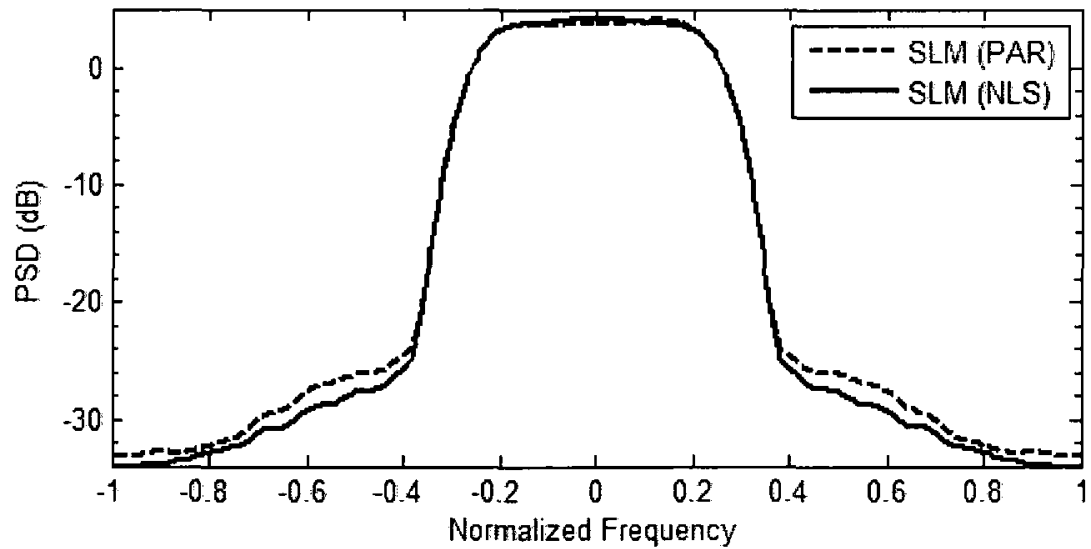

(c)

Fig. 6.12 PSDs of 16-user CDMA signals in presence of PD-HPA at (a) IBO $=1$ $\mathrm{dB}$, (b) IBO $=2 \mathrm{~dB}$, and (c) IBO $=3 \mathrm{~dB}$. 


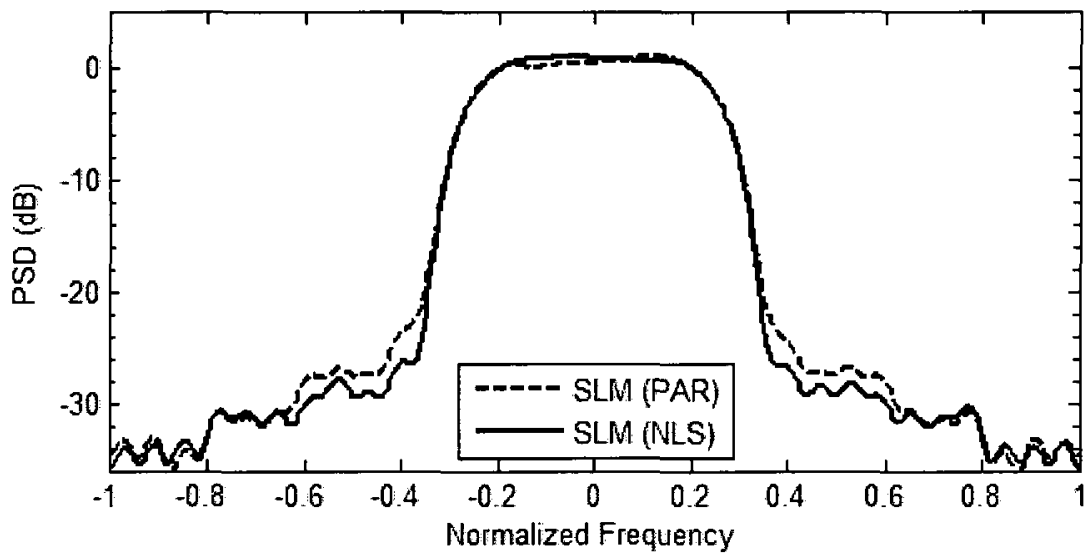

(a)

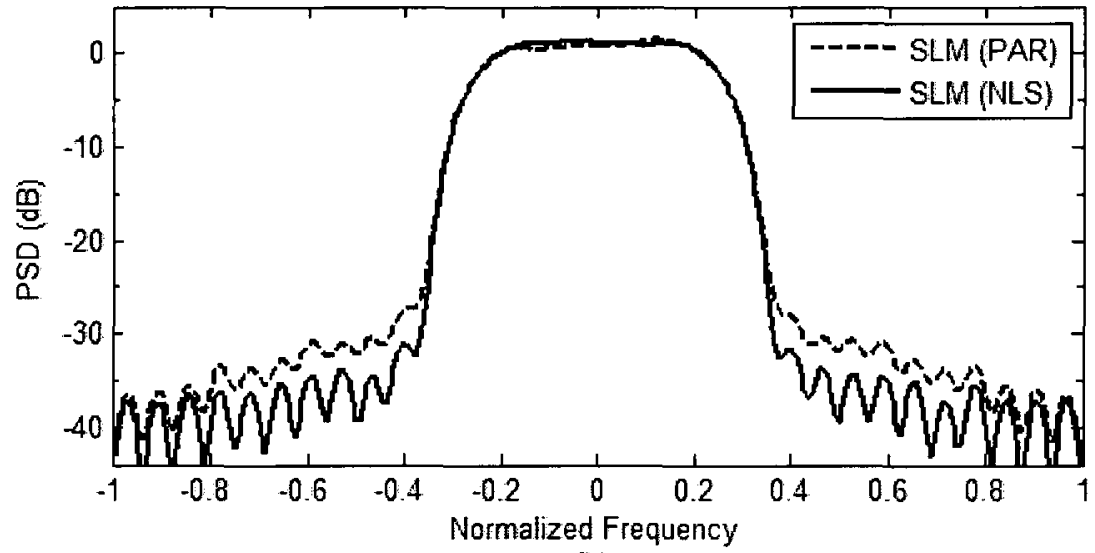

(b)

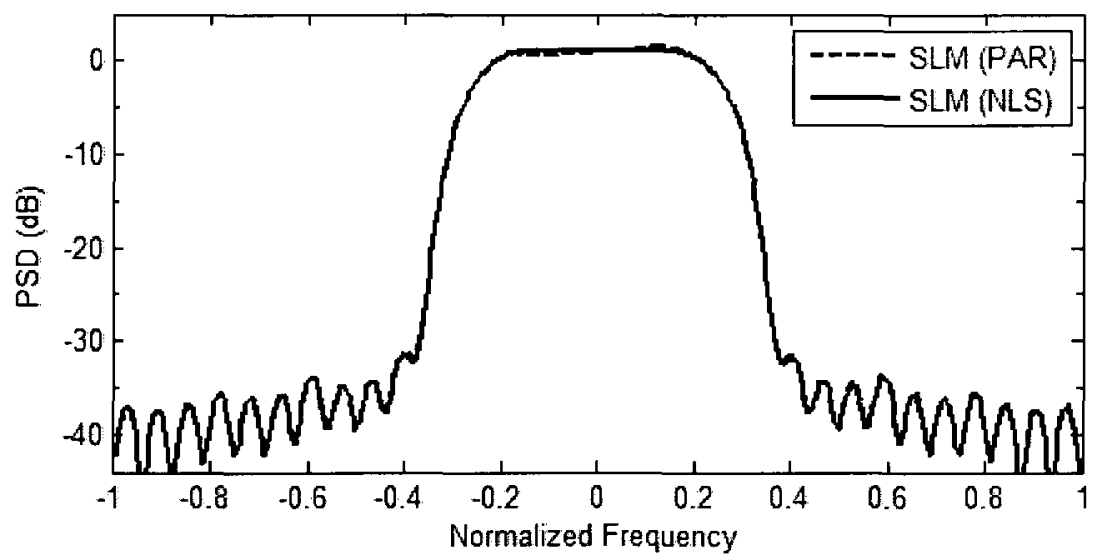

(c)

Fig. 6.13 PSDs of 8-user CDMA signals in presence of PD-HPA at (a) IBO $=1 \mathrm{~dB}$, (b) $\mathrm{IBO}=2 \mathrm{~dB}$, and (c) IBO $=3 \mathrm{~dB}$. 


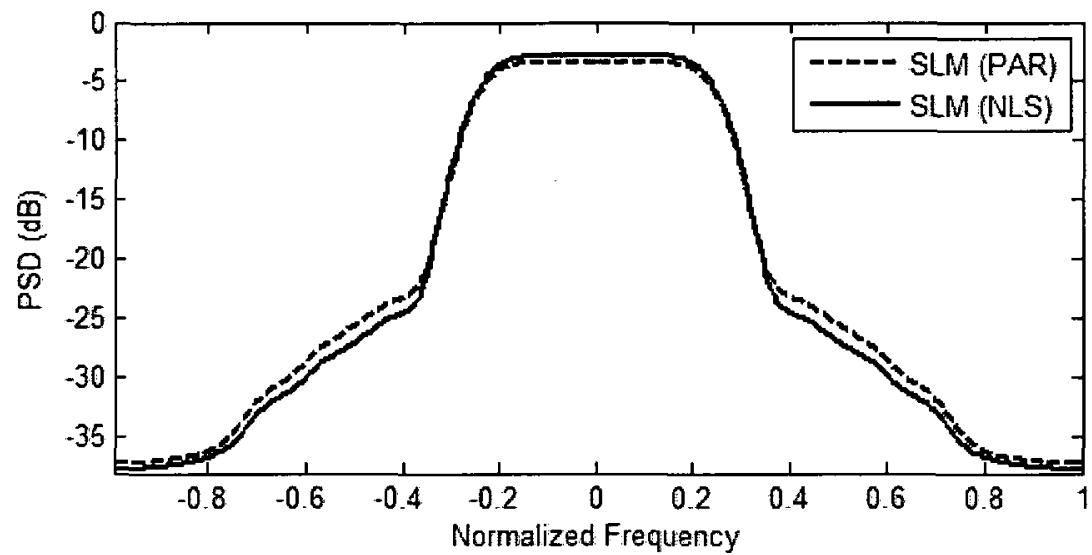

(a)

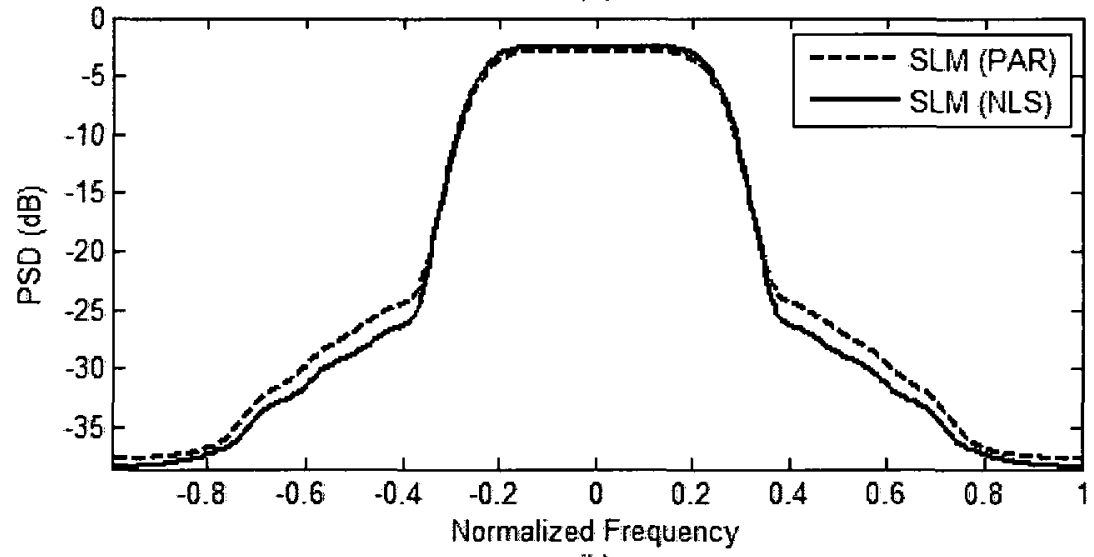

(b)

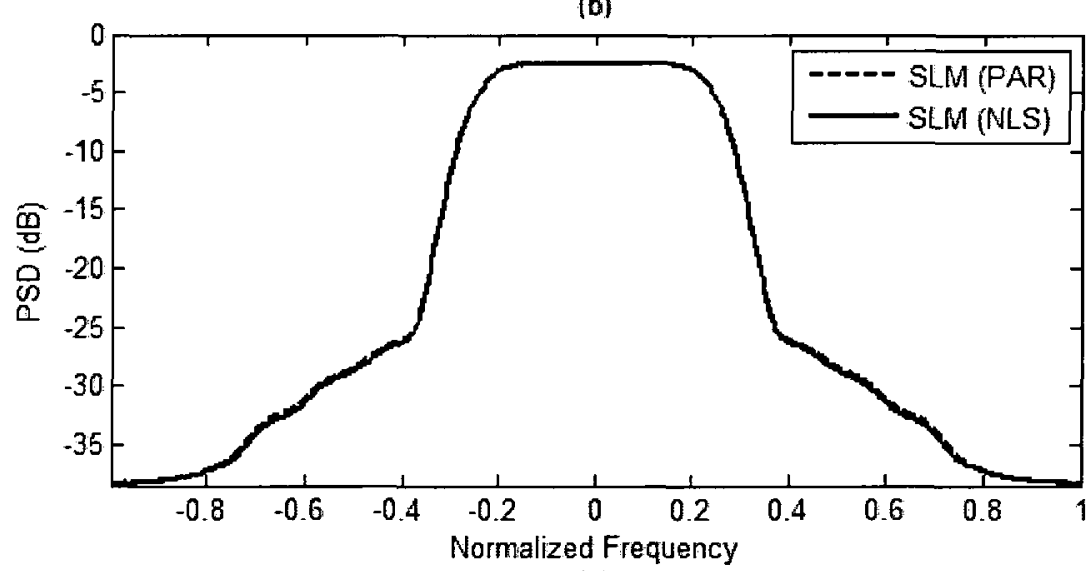

(c)

Fig. 6.14 PSDs of 128-OFDM signals in presence of PD-HPA at (a) IBO $=1 \mathrm{~dB}$, (b) $\mathrm{IBO}=2 \mathrm{~dB}$, and (c) $\mathrm{IBO}=3 \mathrm{~dB}$. 


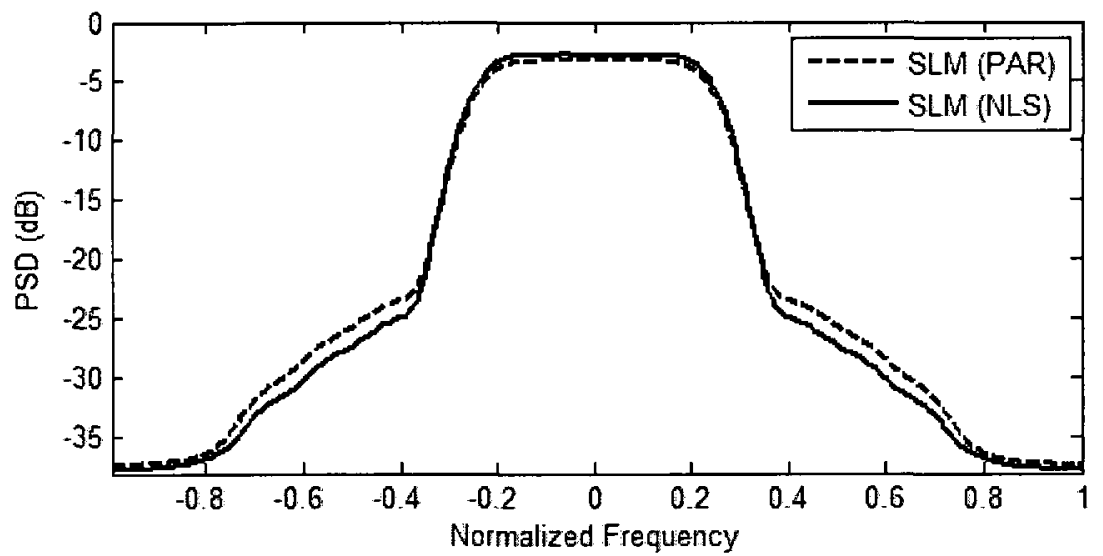

(a)

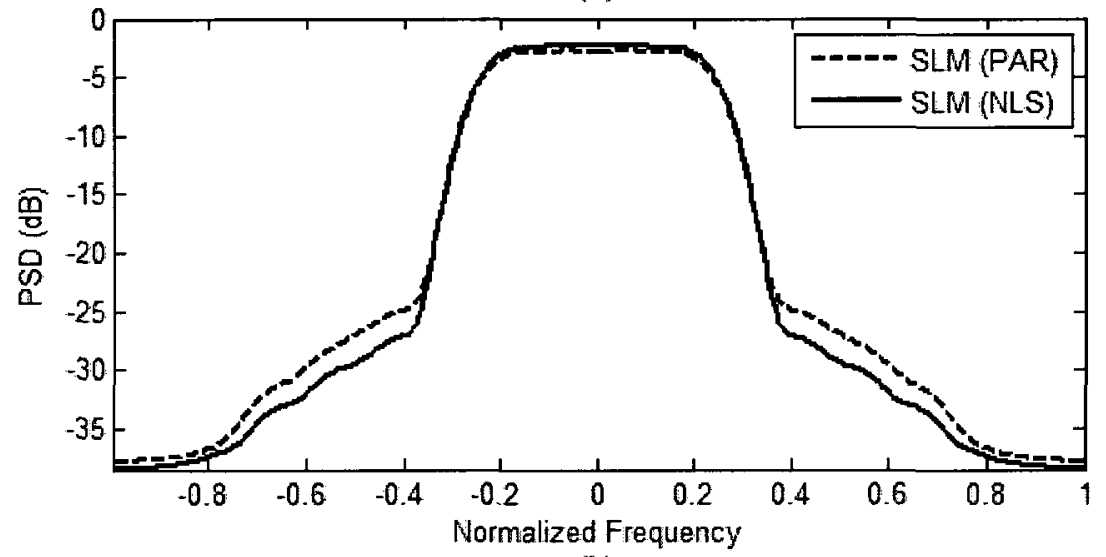

(b)

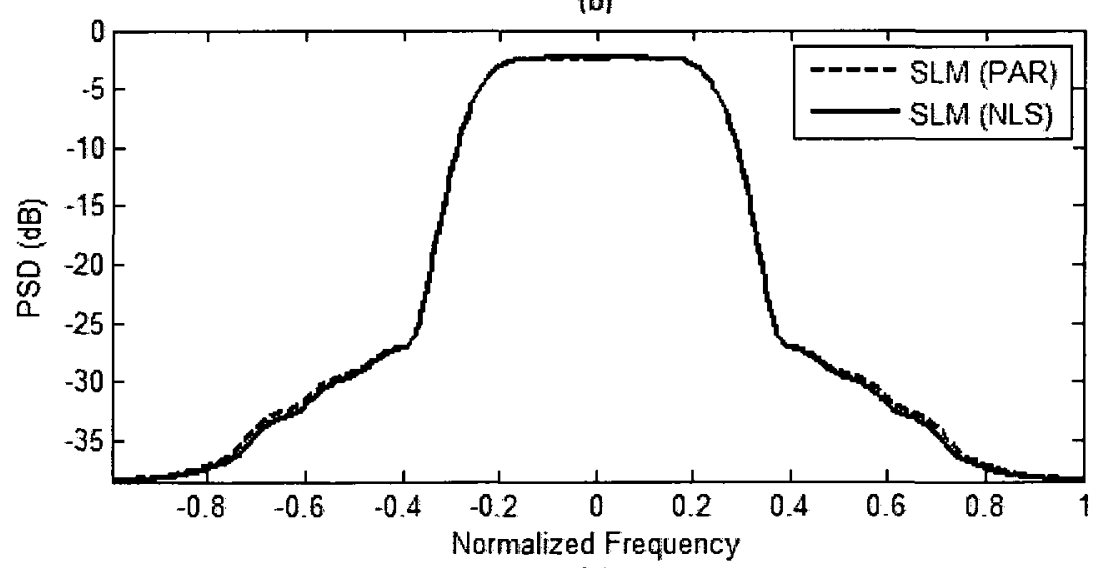

(c)

Fig. 6.15 PSDs of 64-OFDM signals in presence of PD-HPA at (a) IBO $=1 \mathrm{~dB}$, (b) $\mathrm{IBO}=2 \mathrm{~dB}$, and (c) IBO $=3 \mathrm{~dB}$. 


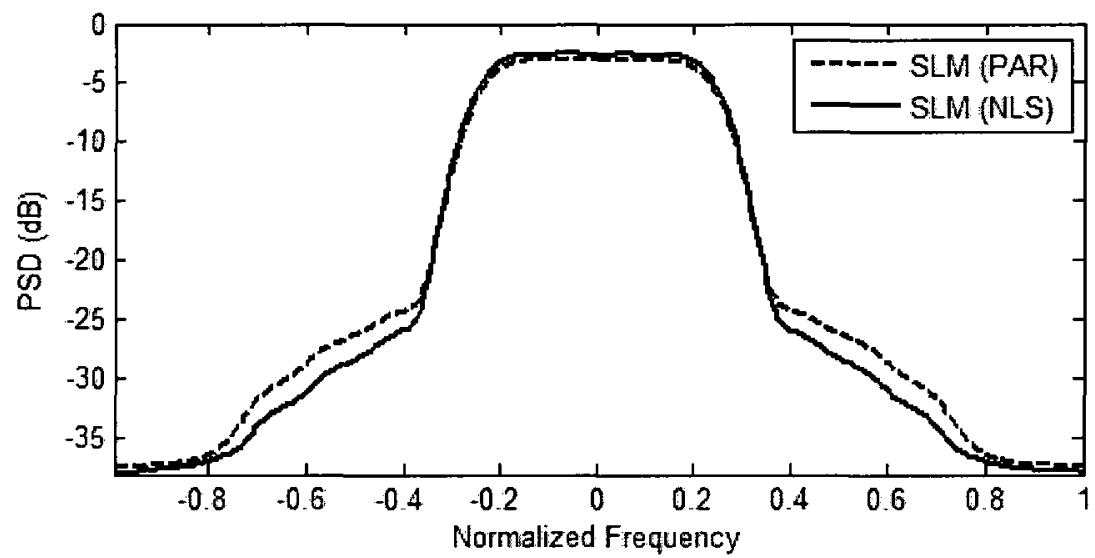

(a)

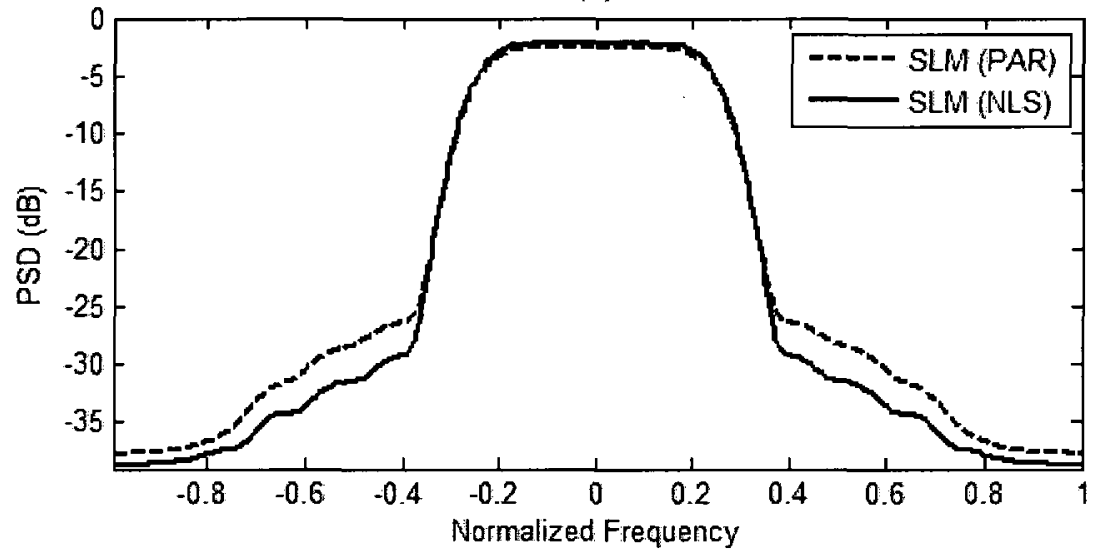

(b)

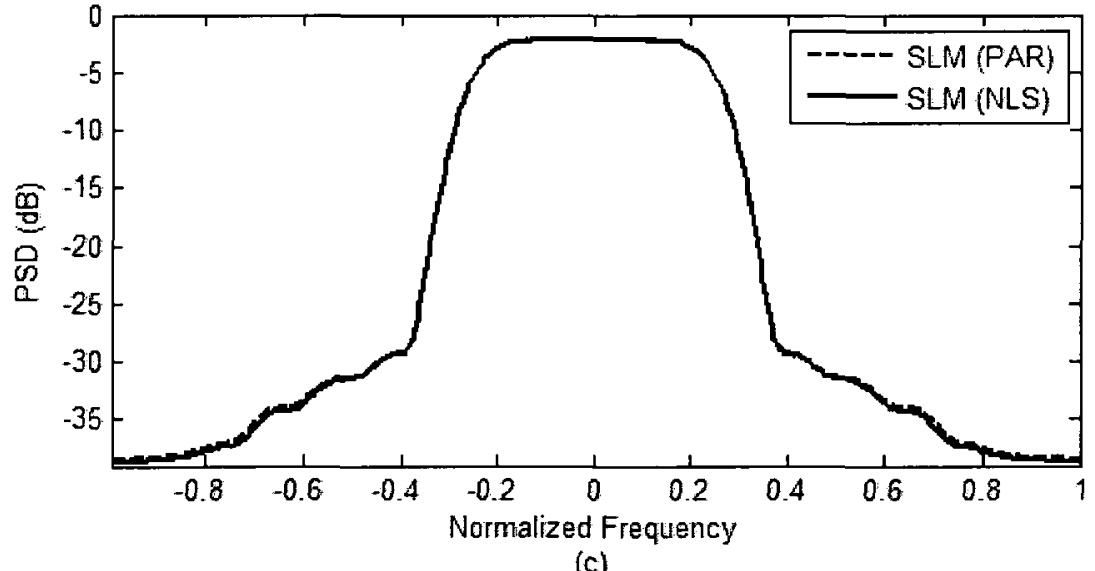

(c)

Fig. 6.16 PSDs of 16-OFDM signals in presence of PD-HPA at (a) IBO $=1 \mathrm{~dB}$, (b) IBO $=2 \mathrm{~dB}$, and (c) $\mathrm{IBO}=3 \mathrm{~dB}$. 
Table 6.2 ACPR for 32-user CDMA in presence of PD-HPA.

\begin{tabular}{|c|c|c|c|}
\hline & \multicolumn{3}{|c|}{ ACPR (dB) } \\
\hline IBO $\gamma(\mathrm{dB})$ & 1 & 2 & 3 \\
\hline No SLM & -25.10 & -26.66 & -28.48 \\
\hline SLM (PAR) & -25.86 & -27.97 & -30.53 \\
\hline SLM (NLS) & $\mathbf{- 2 7 . 3 1}$ & -27.98 & -32.14 \\
\hline Linear & \multicolumn{3}{|c|}{-37.22} \\
\hline
\end{tabular}

Table 6.3 ACPR for 16-user CDMA in presence of PD-HPA.

\begin{tabular}{|c|c|c|c|}
\hline & \multicolumn{3}{|c|}{ ACPR (dB) } \\
\hline IBO $\gamma(\mathrm{dB})$ & 1 & 2 & 3 \\
\hline No SLM & -25.23 & -26.82 & -28.69 \\
\hline SLM (PAR) & -26.18 & -28.18 & -31.18 \\
\hline SLM (NLS) & $-\mathbf{2 7 . 4 2}$ & -29.46 & $-\mathbf{3 2 . 7 1}$ \\
\hline Linear & \multicolumn{3}{|c|}{-37.12} \\
\hline
\end{tabular}

Table 6.4 ACPR for 8-user CDMA in presence of PD-HPA.

\begin{tabular}{|c|c|c|c|}
\hline & \multicolumn{3}{|c|}{ ACPR (dB) } \\
\hline IBO $\gamma(\mathrm{dB})$ & 1 & 2 & 3 \\
\hline No SLM & -25.64 & -26.96 & -28.90 \\
\hline SLM (PAR) & -29.37 & -32.76 & -36.28 \\
\hline SLM (NLS) & $\mathbf{- 3 0 . 8 9}$ & -36.59 & $-\mathbf{3 6 . 3 6}$ \\
\hline Linear & \multicolumn{3}{|c|}{-37.40} \\
\hline
\end{tabular}


Table 6.5 ACPR for 128-OFDM in presence of PD-HPA.

\begin{tabular}{|c|c|c|c|}
\hline & \multicolumn{3}{|c|}{ ACPR (dB) } \\
\hline IBO $\gamma(\mathrm{dB})$ & 1 & 2 & 3 \\
\hline No SLM & -24.93 & -26.42 & -28.13 \\
\hline SLM (PAR) & -25.01 & -26.56 & -28.39 \\
\hline SLM (NLS) & $\mathbf{- 2 5 . 2 2}$ & $\mathbf{- 2 6 . 7 8}$ & $\mathbf{- 2 8 . 6 8}$ \\
\hline Linear & \multicolumn{3}{|c|}{-37.13} \\
\hline
\end{tabular}

Table 6.6 ACPR for 64-OFDM in presence of PD-HPA.

\begin{tabular}{|c|c|c|c|}
\hline & \multicolumn{3}{|c|}{ ACPR (dB) } \\
\hline IBO $\gamma(\mathrm{dB})$ & 1 & 2 & 3 \\
\hline No SLM & -24.94 & -26.44 & -28.17 \\
\hline SLM (PAR) & -24.64 & -26.35 & -28.29 \\
\hline SLM (NLS) & $\mathbf{- 2 5 . 3 6}$ & $\mathbf{- 2 7 . 1 9}$ & $\mathbf{- 2 9 . 3 6}$ \\
\hline Linear & \multicolumn{3}{|c|}{-37.12} \\
\hline
\end{tabular}

Table 6.7 ACPR for 16-OFDM in presence of PD-HPA.

\begin{tabular}{|c|c|c|c|}
\hline & \multicolumn{3}{|c|}{ ACPR (dB) } \\
\hline IBO $\gamma(\mathrm{dB})$ & 1 & 2 & 3 \\
\hline No SLM & -24.97 & -26.50 & -28.31 \\
\hline SLM (PAR) & -25.82 & -27.41 & -30.50 \\
\hline SLM (NLS) & $\mathbf{- 2 5 . 9 8}$ & $\mathbf{- 2 8 . 2 2}$ & $\mathbf{- 3 1 . 2 0}$ \\
\hline Linear & \multicolumn{3}{|c|}{-37.12} \\
\hline
\end{tabular}


It is clear from the PSDs plotted in Fig. 6.11-6.16 and the computed ACPRs tabulated in Table 6.2-6.7 that using the minimum NLS measure adjusted to the IBO level of interest, compared to the minimum PAR as selection criteria to select the signal to be transmitted, leads to less out-of-band emissions. In turn, lower spectral regrowth is achieved. Also, it can be observed that as the IBO level increases, the differences between the performances of the representations selected based on the minimum NLS measure and those selected based on the minimum PAR tend to vanish.

Finally, an important concluding remark regarding the performance of the NLS measure in comparison with other measures as selection criteria in SLM (or any search and optimization technique) should be mentioned here. It is difficult to determine a certain value for the outperformance of the NLS measure over other measures, since the selection process itself may lead to similar representations, depending on the input signal characteristics, resulting in the same performance. Hence, the outperformance can be one in a range of values. For instance, in the BER performance, an SNR reduction from $0 \mathrm{~dB}$ to $x \mathrm{~dB}$ at a certain BER can be achieved and similarly for the ACPR.

\subsection{Performance assessment with different HPA models}

In our approach for developing the NLS measure, we have assumed the nonlinear amplifier chain is a PD-HPA. Such an assumption is quite realistic since a PD-HPA pair has become a common practice in wireless communication to compensate for nonlinear 
distortion due to an HPA and it also represents a cost-effective technique among many HPA linearization techniques $[46,47]$. However, we claim that the NLS measure would also be effective with other amplifier models. Such a claim can be justified through understanding the nonlinear behavior of the HPA in the context of this dissertation in the following subsection.

\subsubsection{Applicability of the NLS measure with other HPA models}

Consider the three primary regions of the AM-AM transfer function of typical HPA models: the linear (or near linear) region, the nonlinear region, and the inversion/saturation region. These three regions for a typical traveling wave tube amplifier (TWTA) and solid state power amplifier (SSPA) are illustrated in Fig. 6.17.

In the near linear region, the output signal from the HPA has minimal distortion that will not negatively affect recovery of the information sent by the $k$ th user at the receiver, unlike in the nonlinear region, where the output signal experiences more nonlinear distortion. In the nonlinear region, the distortion is more severe and the signal values are clustered at the HPA output and become more vulnerable to noise and other channel impairments. However, there is still a one-to-one mapping between the input signal values and the output values. Based on this one-to-one mapping, the nonlinear region can be linearized using any of the linearization schemes available in the literature, such as predistortion. An existing solution is the use of equalization techniques at the 
receiver to compensate for such a nonlinear distortion. On the other hand, for the inversion/saturation region, where the input signal exceeds the threshold $\left(\zeta_{1}\right.$ or $\left.\zeta_{2}\right)$, an output signal value has a many-to-one mapping to the input values, as demonstrated in Fig. 6.17 for the values with the square and circle markers in the case of the TWTA model, and the values with the diamond markers in the case of the SSPA model. In this case, uniquely discriminating between these input values would be challenging, resulting in additional expected errors at the receiver due to erroneous symbol decoding. From this discussion, it is apparent that the severest nonlinear distortion arises when the input signal exceeds the HPA inversion/saturation threshold.

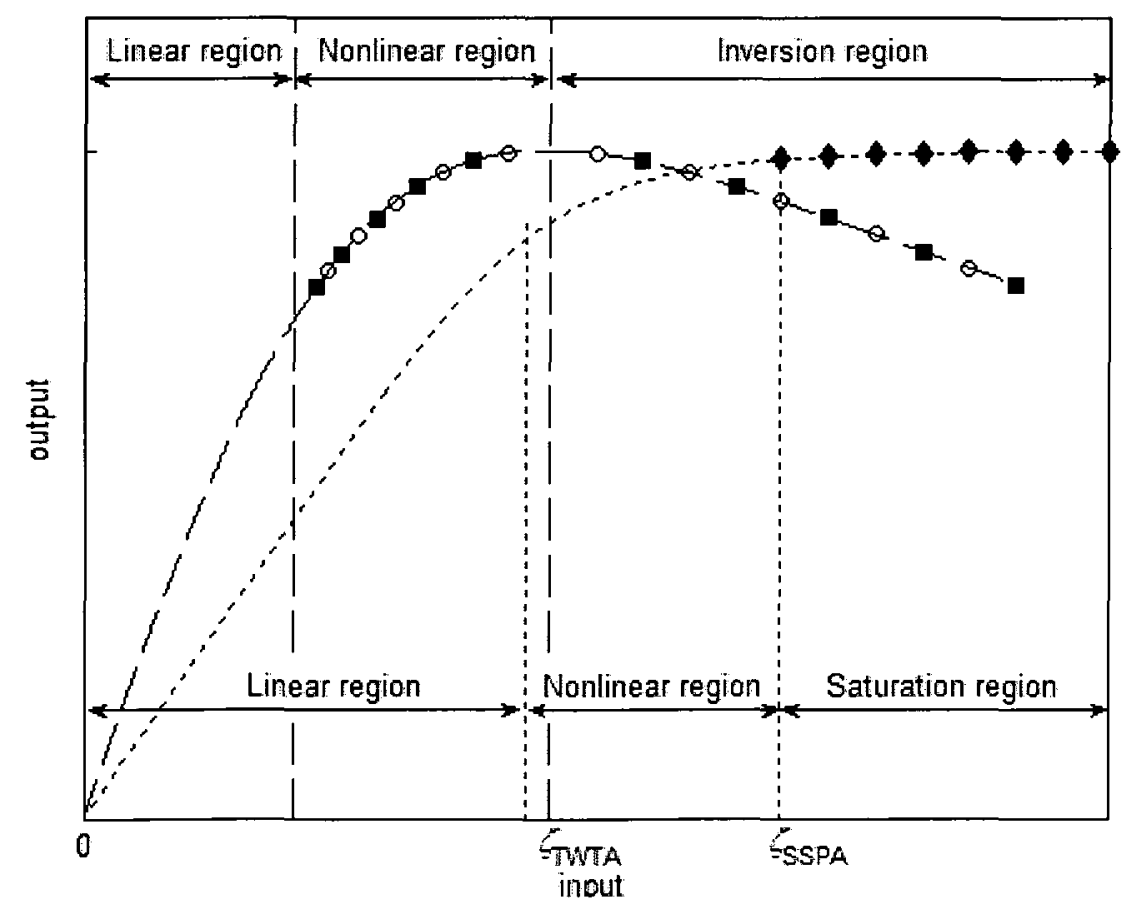

Fig. 6.17 Typical HPA transfer functions for a TWTA and SSPA. 
Accordingly, the NLS measure, combined with a suitable choice of threshold, can be used as an indicator of the severest nonlinear distortion as it quantifies the amount of the signal portion located in the inversion/saturation region, where the data in the transmitted signal become unrecoverable at the receiver. In other words, the reduction of NLS in the input signal to the HPA means trying to grab as much as possible of the signal away from the inversion/saturation region.

In order to examine the performance of the NLS measure with different HPA models, we have considered an example 16-user CDMA signal. Using the SLM technique, two equivalent representations of such a signal are generated based on NLS and PAR as the selection criteria. The TWTA and SSPA are represented by Saleh's model [7] and Rapp's model [29], respectively. Saleh's model is identified by the following AM-AM and AM-PM characteristics:

$$
G[r]=\frac{2 r}{1+r^{2}}
$$

and

$$
\Phi[r]=\frac{\pi}{3} \frac{r^{2}}{1+r^{2}}
$$

While the AM-AM and AM-PM characteristics for Rapp's model are, respectively, given by 


$$
G[r]=\frac{r}{\left(1+r^{4}\right)^{1 / 4}}
$$

and

$$
\Phi[r]=0
$$

For the TWTA model, a reasonable choice for the threshold is the inversion point. For the SSPA model, a reasonable choice for the threshold is the 3-dB compression point, which is defined as the input power at which the gain of the HPA falls by $3 \mathrm{~dB}$ from its linear value. Fig. 6.18 and Fig. 6.19 show the BER performance of a 16-user DS-CDMA system in presence of nonlinearity due to TWTA and SSPA, respectively, where it is clear that SLM based on the minimum NLS shows an improved performance over the minimum PAR at the considered IBO levels. In this case, using the NLS measure as a selection criterion can be considered as a generic suboptimum solution. An optimum solution likely requires the selection criterion to incorporate characteristics of the specific HPA model. 


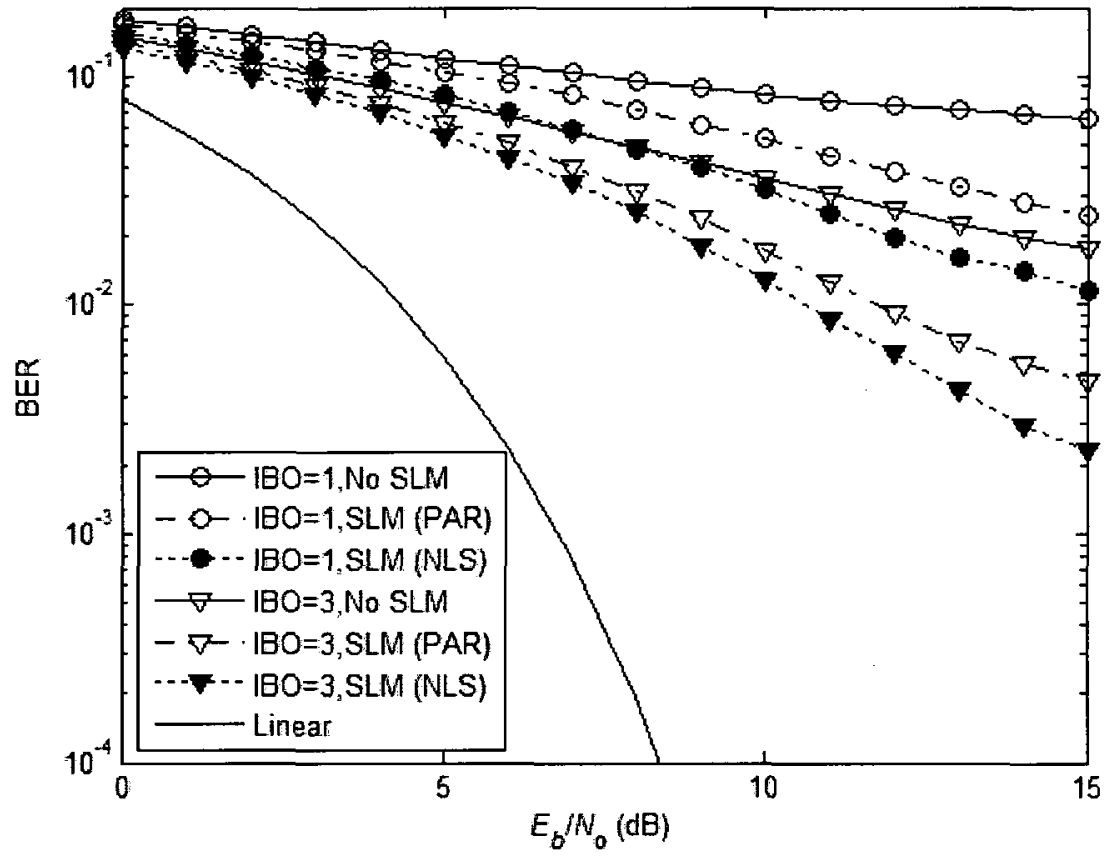

Fig. 6.18 BER performance of 16-user CDMA signals in presence of TWTA.

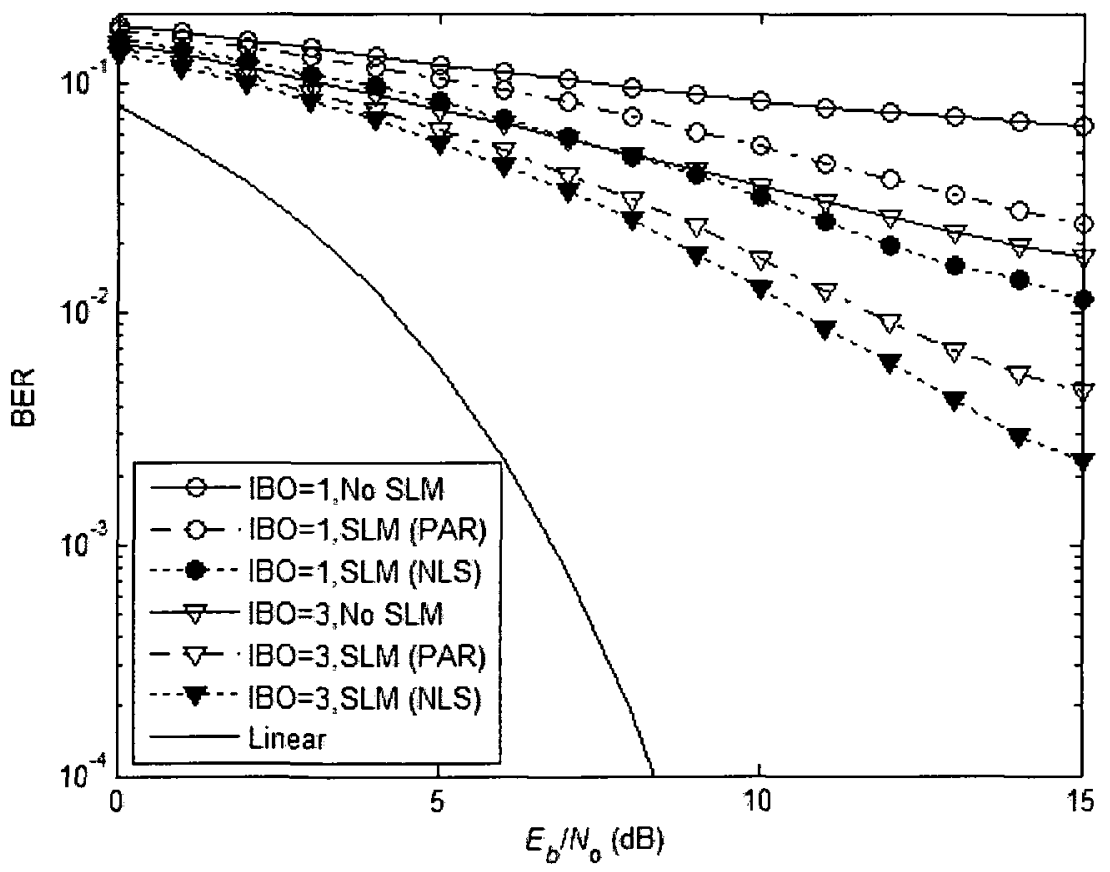

Fig. 6.19 BER performance of 16-user CDMA signals in presence of SSPA. 


\subsection{Conclusions}

In this chapter, the performance of the NLS measure was assessed by applying it as a selection criterion in the SLM technique in comparison with the PAR, where simulation results have shown better performance at all IBO levels of interest. Also, performance assessments have also shown the efficiency of the NLS measure with TWTA and SSPA models. This was justified through the investigation of the transfer function of a typical HPA, where it was apparent that the severest nonlinear distortion arises when the input signal exceeds the inversion/saturation threshold of the HPA. Accordingly, the NLS measure can be used as an indicator of the severest nonlinear distortion as it quantifies the amount of the signal portion that falls in the inversion/saturation region of the HPA's transfer function, where the data in the transmitted signal become unrecoverable at the receiver. 


\section{Chapter 7}

\section{CONCLUSIONS}

\section{AND SUGGESTIONS FOR FUTURE RESEARCH}

$\mathrm{n}$ this chapter, we conclude the dissertation with a summary of the presented
analyses, the established results, and the significance of the contributions drawn from the dissertation. Future areas of research as a follow-up to the work presented in this thesis are also discussed.

\subsection{Conclusions}

In this dissertation, we investigated the nonlinear distortion effects on communication signals passing through a nonlinear amplifier chain. We have considered two of the current most widely used signaling schemes; CDMA and OFDM. These nonlinear distortion effects represent degradation in the overall system performance in terms of BER degradation and additional out-of-band emission (spectral regrowth). The goal behind this investigation was to determine which signal characteristics at the amplifier input represent the main contributors to such effects of nonlinear distortion. We have seen that such an action-oriented research in the input signal to the power amplifier is worthwhile as it seeks to minimize the impact of the nonlinear distortion before the signal even hits the amplifier. Based on the analysis of the nonlinear distortion effects 
(BER degradation and spectral regrowth), we were able to characterize the signal's nonlinear behavior through its statistical characteristics in relation to the amplifier characteristics. In our approach to the analysis and characterization of the nonlinear distortion, some assumptions were considered. The considered CDMA system is assumed synchronous with equal users' powers. A memoryless PD-HPA pair is considered as the amplifier chain, where it has no AM-PM conversion, and has an amplitude transfer function that is linear up to a certain level, after which it saturates (clips) the output signal. An AWGN is considered as the channel impairment. In the following, we summarize the results established in this dissertation.

- The SNR at the detector input of both CDMA and OFDM systems in presence of nonlinear distortion was investigated. SNR expressions were developed as functions of characteristics of the amplifier input signal in relation to the PD-HPA. Through these expressions, the signal characteristics contributing to BER degradation are established. These characteristics are: the rate at which the signal exceeds the saturation threshold and the variance of the signal portion above the threshold [48].

- An SNR relative efficiency was defined in a manner with similarities to the efficiency defined for multiuser communications systems [36, 37]. Defining the relative efficiency leads to further understanding of the amplifier nonlinear distortion and provides a useful way to determine the IBO level required to achieve a certain BER in presence of both AWGN and amplifier nonlinearity. 
- Based on the analysis of the PSD at the PD-HPA output and the piecewise analysis of the clipped signal portion, it was established that the signal characteristics contributing to spectral regrowth are: the variance of the clipped signal portion, the threshold crossing rate, and the mean duration of crossings [105].

- Based on the established signal characteristics that represent the main contributors to BER degradation and spectral regrowth, a new measure, NLS, which better characterizes the input signal to the PD-HPA in relation to the PD-HPA characteristics, was developed. This parameter represents a quality measure for the potential nonlinear distortion. Additionally, being a function in the IBO level gives the NLS measure a superior advantage over the existing measures as it can be adjusted to the IBO level required to work at according to design demands.

- The performance of the NLS measure was assessed by applying it as a selection criterion in the SLM technique in comparison with the PAR, where simulation results have shown better performance at all IBO levels of interest. Also, performance assessments have shown that the NLS measure is also effective with TWTA and SSPA models. This was justified through the investigation of the transfer function of a typical HPA, where it was apparent that the severest nonlinear distortion arises when the input signal exceeds the inversion/saturation threshold of the HPA. Accordingly, the NLS measure can be used as an indicator of the severest nonlinear distortion as it quantifies the amount of the signal portion that falls in the inversion/saturation region 
of the HPA's transfer function, where the data in the transmitted signal become unrecoverable at the receiver.

During the research work in this dissertation, other relevant contributions have been achieved that also have significant practical applications. In the following, we summarize such contributions.

- Synchronous CDMA signals with orthogonal spreading codes were analyzed as a special case. It was shown that the PSD of the CDMA signal depends primarily on the PSDs spreading functions assigned to each user in the system as it is approximately a scaled sum of the PSDs of the spreading functions assigned to all users. For the case of using Walsh codes in synchronous CDMA signals, it was shown that the PSD of Walsh functions and the PSD of the sum of Walsh functions depend on the specific properties of Walsh codes. Based on such properties of Walsh codes, an effective Walsh code assignment algorithm was presented to minimize spectral regrowth [106].

- It has been shown in the literature that the main drawback in search and optimization techniques such as SLM and PTS techniques is the computational complexity and exhaustive search. In this dissertation, we presented a low complexity technique CSS [101], as an alternative to the SLM technique. The concept of the presented technique is based on cyclic shifts of the symbol data sequences, as a replacement of the multiplication by the phase rotation vector, to reduce computational burden. 
Additionally, the search procedure is simplified by adopting a greedy algorithm. In this case, the number of generated representations, among which the best signal is selected, grows linearly with the number of users. As a result, an exhaustive search, among a huge number of representations growing exponentially with the number of users, is averted.

\subsection{Suggestions for future research}

The work presented at this stage of research lays a foundation for analyzing and characterizing nonlinear distortion effects on signals passing through nonlinear amplifier chain. In light of the current contributions, we shed light on some of the opened issues and suggest future areas of research as a follow-up to the work presented as follows.

- The approach in this dissertation provides a potential insight into the interaction between the signal characteristics and the amplifier characteristics, which results in the nonlinear distortion forms; BER degradation and spectral regrowth. Following such an approach, one would be able to control the signal characteristics in order to minimize the NLS measure and, in turn, reduce the severity of such distortion forms before the signal enters the HPA. We have shown one application to do so by using NLS as a selection criterion in the SLM technique. The SLM, like PTS, is flawed by its computational complexity and exhaustive search. Hence, we suggest adopting the NLS minimization in other less complexity techniques such as, for instance, the 
interesting linear block precoding introduced by Falconer [23] that addresses the minimization of the IPV.

- The NLS measure is formulated assuming the nonlinear amplifier chain includes a predistorter, PD-HPA, which is a realistic assumption. Also, we have shown that the NLS measure leads to performance improvement with other amplifier models, with no predistorter involved in the amplifier chain, as it tries to grab most of the HPA input signal to fall before the saturation/inversion region. Hence, it reduces the signal's vulnerability to the severe nonlinear distortion. Unlike the existing nonlinearity measures (PAR, IPV, and CM) that represent generic measures in the sense that they do not take the nonlinear behavior of the signal along the typical transfer function of the HPA into consideration, the NLS measure does characterize such a behavior by classifying the transfer function into two region; before and after the saturation/inversion threshold. However, we may say that the NLS measure is still a generic measure as it does not characterize the signal's nonlinear behavior before the threshold, which depends on the particular HPA characteristics. Therefore, we may also say that the performance improvement achieved by adopting the NLS measure is suboptimal. Hence, seeking the optimum performance requires accurate characterization of the signal's behavior in such a region. Actually, such a research avenue is very challenging and poses an analysis paradox to find a measure that characterizes the signal's nonlinear behavior along its dynamic range and, at the same time, is suitable for all HPA models. 
- The presented analyses were conducted under the assumption of memoryless nonlinearity. Memory nonlinear effect causes scattering and/or hysteresis of the AM$\mathrm{AM}$ and AM-PM curves, and asymmetry in the IMD products. Consequently, more sophisticated techniques that consider the frequency dependent behavior of the HPA are required to analyze and characterize its effect on the HPA input signal. 
Appendix $A$

\section{VARIANCE OF THE DERIVATIVE OF A RANDOM PROCESS WITH RECTANGULAR PASSBAND}

To get the variance of the derivative of a random process, it is convenient to introduce spectral moments since each moment of order $2 i$ is equal to the variance of the $i$ th derivative of the process [62];

$$
\lambda_{2 i}=\int_{-\infty}^{\infty} \omega^{2 i} S_{x x}(\omega) d \omega=\sigma_{x^{(i)}}^{2}
$$

in which $x^{(i)}$ denotes the $i$ th derivative of $x(t)$. Therefore, the variance of a derivative can be computed by integration in the frequency domain, which is much simpler than differentiating a process' variance in the time domain.

Since the PSD $S_{x x}(\omega)$ of the baseband filtered CDMA signal is nearly rectangular, it can be assumed constant over the entire bandwidth $B$ and zero outside such a band as follows

$$
S_{x x}(\omega) \cong\left\{\begin{aligned}
\lambda_{0} / B, & |\omega| \leq B / 2 \\
0, & \text { otherwise }
\end{aligned}\right.
$$


where $B \cong 2 \pi / T_{c}=2 \pi L / T$. Therefore, substituting $S_{x x}(\omega)$ of (A.2) in (A.1) for $i=0$ and 1 , gives

$$
\sigma_{\dot{x}}^{2} \cong \frac{B^{2}}{12} \sigma_{x}^{2} .
$$


Appendix $B$

JOINT PROBABILITY DENSITY FUNCTION OF

\section{THE ENVELOPE AND ITS FIRST DERIVATIVE OF}

\section{COMPLEX GAUSSIAN PROCESSES}

Consider $s(t)=x(t)+j y(t)$ is a zero-mean complex Gaussian process. Since $x(t)$ and $y(t)$ are zero-mean Gaussian processes with variances $\sigma_{x}^{2}=\sigma_{y}^{2}$, their derivatives $\dot{x}(t)$ and $\dot{y}(t)$ are also zero-mean Gaussian processes with variances $\sigma_{\dot{x}}^{2}=$ $\sigma_{\dot{y}}^{2}=E\left\{\dot{x}^{2}(t)\right\}$. Consider the zero-mean Gaussian random vector $\mathbb{X}=[x(t) \dot{x}(t) y(t) \dot{y}(t)]^{T}$ whose pdf can be written as $[60]$

$$
f_{X}(\mathbb{X})=\frac{1}{4 \pi^{2}|\mathbb{K}|^{1 / 2}} \exp \left(-\frac{1}{2} \mathbb{X}^{T} \mathbb{K}^{-1} \mathbb{X}\right)
$$

in which $\mathbb{K}=E\left\{\mathbb{X X}^{T}\right\}$ is the covariance of $\mathbb{X}$. Since $x(t)$ and $y(t)$ are uncorrelated and $E\{x \dot{x}\}=0$, this covariance matrix can be written as

$$
\mathbb{K}=\left[\begin{array}{cccc}
\sigma_{x}^{2} & 0 & 0 & 0 \\
0 & \sigma_{\dot{x}}^{2} & 0 & 0 \\
0 & 0 & \sigma_{x}^{2} & 0 \\
0 & 0 & 0 & \sigma_{\dot{x}}^{2}
\end{array}\right]
$$


and its determinant and its inverse are, respectively, given by

$$
|\mathbb{K}|=\sigma_{\dot{x}}^{4} \sigma_{\dot{x}}^{4}
$$

and

$$
\mathbb{K}^{-1}=\left[\begin{array}{cccc}
\frac{1}{\sigma_{x}^{2}} & 0 & 0 & 0 \\
0 & \frac{1}{\sigma_{\dot{x}}^{2}} & 0 & 0 \\
0 & 0 & \frac{1}{\sigma_{x}^{2}} & 0 \\
0 & 0 & 0 & \frac{1}{\sigma_{\dot{x}}^{2}}
\end{array}\right] .
$$

Since $s(t)=r(t) e^{j \theta(t)}=x(t)+y(t)$, therefore $x(t)$ and $y(t)$ can be written in the polar coordinate as

$$
x(t)=r(t) \cos \theta(t)
$$

and

$$
y(t)=r(t) \sin \theta(t)
$$

Therefore, the pdf of the random vector $\mathbb{V}=[r(t) \dot{r}(t) \theta(t) \dot{\theta}(t)]^{T}$ corresponding to $\mathbb{X}$ is given by 


$$
f_{\mathbb{V}}(\mathbb{V})=|J| f_{\mathbb{X}}(\mathbb{X})
$$

where

$$
J=\left|\frac{\partial \mathbb{X}}{\partial \mathbb{V}}\right|=r^{2}
$$

is the Jacobian determinant of the transformation. It can be found from (B.5) and (B.6) that $x^{2}+y^{2}=r^{2}$ and $\dot{x}^{2}+\dot{y}^{2}=\dot{r}^{2}+r \dot{\theta}^{2}$. Substituting in (B.7), gives

$$
\begin{aligned}
f_{\mathbb{V}}(\mathbb{V}) & \left.=f_{\mathrm{V}}(r, \dot{r}, \theta, \dot{\theta})\right) \\
& =\frac{1}{4 \pi^{2} \sigma_{x}^{2} \sigma_{\dot{x}}^{2}} \exp \left(-\frac{1}{2}\left(\frac{r^{2}}{\sigma_{x}^{2}}+\frac{\dot{r}^{2}}{\sigma_{\dot{x}}^{2}}+\frac{r^{2} \dot{\theta}^{2}}{\sigma_{\dot{x}}^{2}}\right)\right) .
\end{aligned}
$$

Integrating $\theta$ from 0 to $2 \pi$ and $\dot{\theta}$ from $-\infty$ to $\infty$, we obtain the joint pdf of $r$ and $\dot{r}$ as

$$
f_{r, \dot{r}}(r, \dot{r})=\frac{r}{\sqrt{2 \pi \sigma_{x}^{4} \sigma_{\dot{x}}^{2}}} \exp \left(-\frac{1}{2}\left(\frac{r^{2}}{\sigma_{x}^{2}}+\frac{\dot{r}^{2}}{\sigma_{\dot{x}}^{2}}\right)\right)
$$




\section{REFERENCES}

[1] G. Tong Zhou, H. Qian and N. Chen, Communication System Nonlinearities: Challenges and Some Solutions, in S. Marshall and G. L. Sicuranza., Advances in Nonlinear Signal and Image Processing, Hindawi, pp. 141-167, 2006.

[2] L. Frenzel, "Efficient digital RF power amp slashes basestation power, size, and cost," J. Electronic Design, vol. 54, no. 9, pp. 34-34, Apr 2006.

[3] D. Falconer, "Adaptive equalization of channel nonlinearities in QAM data transmission systems," Bell Syst. Tech. J., vol. 57, no. 7, pp. 2589-2611, Sept. 1978.

[4] S. Benedetto and E. Biglieri, "Nonlinear equalization of digital satellite channels," IEEE J. Select. Areas Commun., vol. 1, no. 1, pp. 57-62, Jan. 1983.

[5] G. Karam and H. Sari, "Analysis of predistortion, equalization, and ISI cancellation techniques in digital radio systems with nonlinear transmit amplifiers," IEEE Trans. Commun., vol. 37, no.12, pp. 1245-1253, Dec. 1989.

[6] A. Saleh and J. "Salz, Adaptive linearization of power amplifiers in digital radio systems," Bell Syst. Tech. J., vol. 62, no. 4, pp. 1019-1033, Apr. 1983. 
[7] A. M. Saleh, "Frequency independent and frequency dependent nonlinear models of TWT amplifiers," IEEE Trans. Commun., vol. COM-29, no. 11, pp. 1715$1720,1981$.

[8] X. Li and L. J. Cimini, Jr, "Effects of clipping and filtering on the performance of OFDM," IEEE Commun.. Lett., vol. 2, no. 5, May, 1998, pp. 131-133.

[9] Olli Vaananen, Jouko Vankka, and Kari Halonen, "Effect of baseband clipping in wideband CDMA system," in Proc. IEEE 7th Int. Symp. Spread Spectrum Tech.and App., vol. 2, 2002, pp. 445-449.

[10] S. C. Thompson, J. G. Proakis, and J. R. Zeidler, "The effectiveness of signal clipping for PAPR and total degradation reduction in OFDM systems," in Global Telecommun. Conf., GLOBECOM'05, vol. 5, 2005, pp. 2807-2811.

[11] X. Wang, T. Tjhung, and C. S. Ng, "Reduction of peak-to-average power ratio of OFDM system using a companding technique," IEEE Trans. Broadcast., vol. 45, no. 3, pp. 303-307, Sept. 1999.

[12] T. Jiang, Y. Yang, and Y.Song, "Exponential companding technique for PAPR reduction in OFDM systems," IEEE Trans. Broadcast., vol. 51, no. 2, pp. 244248, Jun. 2005. 
[13] S. A. Aburakhia, E. F. Badran, and D. A. E. Mohamed, "Linear companding transform for the reduction of peak-to-average power ratio of OFDM signals," IEEE Trans. Broadcast., vol. 55, no. 1, pp. 155-160, Mar. 2009.

[14] R. Bauml, R. F. H. Fischer, and J. B. Huber, "Reducing the peak-to-average power ratio of multicarrier modulation by selected mapping," IEE Electron. Lett., vol. 32 , no. 22 , pp. $2056-2057$, Oct. 1996.

[15] K. Sathananthan and C. Tellambura, "Peak-to-average power ratio analysis in multicode CDMA," in Proc. IEEE 56th Veh. Technol. Conf., vol. 1, Sept. 2002, pp. 500-504.

[16] R. J. Baxley, C. Zhao, and G. T. Zhou, "Magnitude-scaled selected mapping_a crest factor reduction scheme," in Proc. IEEE Intern. Conf. on Acoustics, Speech, and Signal Process. ICASSP 2007, vol. 3, 2007, pp. 141-144.

[17] S. G. Kang, J. G. Kim, and E. K. Joo, "A novel subblock partition scheme for partial transmit sequence OFDM," IEEE Trans. Broadcast., vol. 45, no. 3, pp. 333-338, Sept. 1999.

[18] L. J. Cimini and N. R Sollenberger, "Peak-to-average power ratio reduction of an OFDM signal using partial transmit sequences with embedded side information," in Proc. IEEE GLOBECOM, vol. 2, Nov. 2000, pp. 746-750. 
[19] W. S. Ho, A. S. Madhukumar, and F.Chin, "Peak-to-average power reduction using partial transmit sequences: A suboptimal approach based on dual layered phase sequencing," IEEE Trans. Broadcast, vol. 49, no. 2, pp. 225-231, Jun. 2003.

[20] V. Lau, "On the analysis of peak-to-average ratio (PAR) for IS95 and CDMA2000 systems," IEEE Trans. Veh. Technol., vol. 49, no. 6, pp. 2174-2188, Nov. 2000.

[21] H. Ochiai and H. Imai, "On the distribution of peak-to-average power ratio in OFDM signals," IEEE Trans. Commun., vol. 49, no. 2, pp. 282-289, Feb. 2001.

[22] A. Behravan and T. Eriksson, "Some statistical properties of multicarrier signals and related measures," in Proc. IEEE Veh. Technol. Conf., 2006, pp. 1854-1858.

[23] D. Falconer, "Linear precoding of OFDMA signals to minimize their instantaneous power variance," in Proc. IEEE Wireless Comm. and Networking Conf., Apr. 2010.

[24] He Heng and Yang Xiao Yun, "Cubic metric and PAPR of OFDM-CPM signal," in Proc. 4th Int. Conf. on Wireless Commun., Networking and Mobile Computing WiCOM'08, Oct. 2008. pp. 1-3. 
[25] R. Blum and M. Jeruchim, "Modeling nonlinear amplifiers for communication simulation," in Proc. IEEE Int. Conf. Commun. ICC'89, Jun. 1989. vol. 3, pp. 1468-1472.

[26] W. Bosch and G. Gatti, "Measurement and simulation of memory effects in predistortion linearizers," IEEE Trans. Microwave Theory and Tech., vol. 37, no. 12 , pp. $1885-1890,1989$.

[27] J. H. K. Vuolevi, T. Rahkonen, and J. P. A.Manninen, "Measurement technique for characterizing memory effects in RF power amplifiers," IEEE Trans. Microwave Theory and Tech., vol. 49, no. 8, pp. 1383-1389, 2001.

[28] R. Raich and G. T. Zhou, "On the modeling of memory nonlinear effects of power amplifiers for communication applications," in Proc. IEEE 10th Digital Signal Process. Workshop DSP '02, pp. 7-10, Pine Mountain, Ga, USA, Nov. 2002.

[29] C. Rapp, "Effects of HPA-nonlinearity on a 4-DPSK/OFDM-signal for a digital sound broadcasting system," in Proc. 2nd European Conference on Satellite Communications, 1991.

[30] A. J. Cann, "Nonlinearity model with variable knee sharpness," IEEE Trans. Aerosp. Electron. Syst., vol. AES-16, no. 6, pp. 874-877, 1980.

[31] S. C. Cripps, RF Power Amplifier for Wireless Communications, Artech House, Norwood, MA, USA, 1999. 
[32] A. Katz, "Linearization: reducing distortion in power amplifiers," IEEE Microwave Mag., vol. 2, no. 4, pp. 37-49, Dec. 2001.

[33] G. T. Zhou, H. Qian, L. Ding, and R. Raich, "On the baseband representation of a bandpass nonlinearity," IEEE Trans. Signal Process., vol. 53, no. 8, part 1, pp. 2953-2957, 2005.

[34] R. De Gaudenzi and F. Giannetti, "DS-CDMA satellite diversity reception for personal satellite communication: satellite-to-mobile link performance analysis," IEEE Trans. Veh. Technol., vol. 47, no. 2, pp. 658-672, May 1998.

[35] A. Conti, D. Dardari, and V. Tralli, "An analytical framework for CDMA systems with a nonlinear amplifier and AWGN," IEEE Trans. Commun., vol. 50, no. 7, pp. 1110-1120, Jul. 2002.

[36] J. Proakis, Digital Communications, $4^{\text {th }}$ ed., New York: McGraw-Hill Companies, Inc., Boston, 2001.

[37] Sergio Verdu, Multiuser Detection, New York: Cambridge University Press, 1998.

[38] R. W. Chang, "Synthesis of band-limited orthogonal signals for multichannel data transmission," Bell Syst. Tech. J., vol. 45, pp. 1775-1796, Dec. 1966. 
[39] S. B. Weinstein and P. M. Ebert, "Data transmission by frequency-division multiplexing using the discrete Fourier transform," IEEE Trans. Commun., vol. COM-19, pp. 628-634, Oct. 1971.

[40] A. Peled and A. Ruiz, "Frequency domain data transmission using reduced computational complexity algorithms," in Proc. IEEE Int. Conf. Acoust., Speech, Signal Process., Denver, 1980, pp. 964-967.

[41] Slawomir and Pietrzyk, OFDMA for Broadband Wireless Access, USA : Artech House Inc., 2006.

[42] Ramjee Prasad, OFDM for Wireless Communications Systems, Artech House universal personal communications series, Boston, 2004.

[43] L. Hanzo and T. Keller, OFDM and MC-CDMA-A Primer, John Wiley \& Sons, Ltd, 2006.

[44] Davide Dardari, Velio Tralli, and Alessandro Vaccari, "A theoretical characterization of nonlinear distortion effects in OFDM systems," IEEE Trans. Commun., vol. 48, no. 10, pp. 1755-1764, Oct. 2000.

[45] W.Y. Zou and Y. Wu, "COFDM: An overview," IEEE Trans. Broadcast., vol. 41, no. 1, pp. 1-8, Mar. 1995. 
[46] L. Ding, G. T. Zhou, D. R. Morgan, et al., "A robust digital baseband predistorter constructed using memory polynomials," IEEE Trans. Commun., vol. 52, no. 1, pp. 159-165, 2004.

[47] N. Chen, G. Zhou, and H. Qian, "Power efficiency improvements through peakto-average power ratio reduction and power amplifier linearization," in Proc. EURASIP J. Advances in Signal Process., vol. 2007, article ID 20463, 2007.

[48] T. Helaly, R. Dansereau, and M. El-Tanany, "Analysis of BER performance in presence of nonlinear distortion due to PD-HPA in downlink DS-CDMA signals," IEEE Commun. Lett., vol. 14, no. 4, pp. 273-275, Apr. 2010.

[49] Simon Haykin, An Introduction to Analogue and Digital Communications, 2nd ed. Wiley, 2006.

[50] R. Dinis and A. Palhau, "A class of signal-processing schemes for reducing the envelope fluctuations of CDMA signals," IEEE Trans. Commun., vol. 53, no. 5, pp. 882-889, May 2005.

[51] L. Rugini, P. Banelli, and S. Cacopardi, "Theoretical analysis and performance of the decorrelating detector for DS-CDMA signals in nonlinear channels," IEEE Trans. Wireless Commun., vol. 3, no. 2, pp. 367-372, Mar. 2004. 
[52] P. Banelli, and S. Cacopardi, "Theoretical analysis and performance of OFDM signals in nonlinear AWGN channels," IEEE Trans. Commun., vol. 48, no. 3, pp. 430-441, Mar. 2000.

[53] E. Geraniotis and M. Pursley, "Error probability for direct-sequence spread spectrum multiple access communications-Part II: Approximations," IEEE Trans. Commun., vol. COM-30, pp. 985-995, May 1982.

[54] R. Skaug and J. F. Hjelmstad, Spread Spectrum in Communications, London, U.K.: Peter Peregrinus Ltd., 1985.

[55] G. Zhou, "Analysis of spectral regrowth of weakly nonlinear power amplifiers," IEEE Commun. Lett., vol. 4, no. 11, pp. 357-359, Nov. 2000.

[56] G. Zhou, "Predicting spectral regrowth of nonlinear power amplifiers," IEEE Trans. Commun.., vol. 50, no. 5, pp. 718-722, May 2002.

[57] V. Aparin, "Analysis of CDMA signal spectral regrowth and waveform quality," IEEE Trans. Microwave Theory and Tech., vol. 49, no. 12, pp. 2306-2314, Dec. 2001.

[58] P. Banelli, and S. Cacopardi, "Theoretical analysis and performance of OFDM signals in nonlinear AWGN channels", IEEE Trans. Commun., vol. 48, no. 3, pp. 430-441, Mar. 2000. 
[59] K. Gard, L. Larson, and M. Steer, "The impact of RF front-end characteristics on the spectral regrowth of communications signals," IEEE Trans. Microwave Theory and Tech., vol. 53, no. 6, pp. 2179-2186, Jun. 2005.

[60] A. Papoulis, Probability, Random Variables, and Stochastic Processes, $3^{\text {rd }}$ ed., McGraw-Hill, Inc., 1991.

[61] S. O. Rice, "Distribution of the duration of fades in radar transmission: Gaussian noise model," Bell Syst. Tech. J., vol. 37, pp. 581-635, May 1958.

[62] G. Michaelov, S. Sarkani and L. D. Lutes, "Spectral characteristics of nonstationary random processes - a critical review," Structural Safety, vol. 21, no. 3, pp. 223-244, Sept. 1999.

[63] D. R. Morgan, "On level-crossing excursions of Gaussian low-pass random processes," IEEE Trans. Signal Process., vol. 55, no. 7, pp. 3623-3632, Jul. 2007.

[64] J. E. Mazo, "Asymptotic distortion spectrum of clipped, dc-biased, Gaussian noise," IEEE Trans. Commun., vol. 40, no. 8, pp. 1339-1334, Aug. 1992.

[65] M. D. Yacoub, C. R. C. M. da Silva, and J. E. Vargas B., "Level crossing rate and average fade duration for pure selection and threshold selection diversitycombining systems," Int. J. Commun. Syst., 14(10), pp. 897-907, Dec. 2001. 
[66] A. Abdi, K. Wills, H. A. Barger, M. S. Alouini, and M. Kaveh, "Comparison of the level crossing rate and average fade duration of Rayleigh, Rice and Nakagami fading models with mobile channel data," in Proc. IEEE $52^{\text {nd }}$ Veh. Technol. Conf., vol. 4 , Sept. 2000 , pp. $1850-1857$.

[67] K. G. Gard, H. M. Gutierrez, and M. B. Steer, "Characterization of spectral regrowth in microwave amplifiers based on the nonlinear transformation of a complex Gaussian process," IEEE Trans. Microwave Theory and Tech., vol. 47, no. 7, pp. 1059-1069, Jul. 1999.

[68] C. Chien, Digital radio systems on a chip-A system approach, Kluwer Academic Publishers, Norwell, MA, USA, 2000.

[69] R. N. Braithwaite, "Using Walsh code selection to reduce the power variance of band-limited forward-link CDMA waveforms," IEEE J. Select. Areas Commun., vol. 18 , no. 11 , pp. $2260-2269$, Nov. 2000.

[70] A. Shanbhag and E. Tiedemann, "Peak-to-average reduction via optimal Walsh code allocation in third generation CDMA systems", IEEE $6^{\text {th }}$ Intern. Symp. on Spread Spectrum Techniques \& Applications, vol. 2, Sept. 2000, pp. 560-564.

[71] I. M. Hussain, I.A. Tasadduq, "PAPR analysis in OFDM signals based on power variance," in Proc. 4th Int. Conf. on Wireless Commun., Networking and Mobile Computing WiCOM'08, Oct. 2008. pp. 1-4. 
[72] 3GPP TSG-RAN WG1 LTE, Motorola, "Cubic metric in 3GPP LTE," Tdoc R1060023, Tan. 2006.

[73] M. Deumal, A. Behravan, T. Eriksson, and J. L. Pijoan, "Evaluation of performance improvement capabilities of PAPR-reducing methods," Wireless Pers. Commun., vol.47, no. 1, pp. 137-147, 2008.

[74] H. Wu-Zhou and Y. Ming-shun, "Statistical property of threshold-crossing for zero-mean-valued, narrow-banded Gaussian processes," J. Appl. Math. Mech., vol. 22 , no. 6 , pp. 701-710, Jun. 2001.

[75] J. Armstrong, "Peak-to-average power reduction for OFDM by repeated clipping and frequency domain filtering," Electron. Lett., vol. 38, no. 5, pp. 246-247, Feb. 2002.

[76] H. J. Kim, S. C. Cho, H. S. Oh, and J. M. Ahn, "Adaptive clipping technique for reducing PAPR on OFDM systems," in Proc. IEEE 58th Veh. Technol. Conf., vol. 3, pp. 1478-1481, Fall. 2003.

[77] L. Wang and C. Tellambura, "A simplified clipping and filtering technique for PAR reduction in OFDM systems," IEEE Signal Process. Lett., vol. 12, no. 6, pp. 453-456, Jun. 2005. 
[78] R. J. Baxley, C. Zhao, and G. T. Zhou, "Constrained Clipping for Crest Factor Reduction in OFDM," IEEE Trans. Broadcast.., vol. 52, no. 4, pp. 570-575, Dec. 2006.

[79] W. Hafiza and W. Hassan, "Performance analysis and optimization of orthogonal frequency division multiplexing system with enhanced clipping technique," in Proc. Asia-Pacific Conf. on Applied Electromagnetics, APACE 2007, pp. 1-5, Dec. 2007.

[80] L. Wang, and C. Tellambura, "Analysis of clipping noise and tone-reservation algorithms for peak reduction in OFDM systems," IEEE Trans. Veh. Technol., vol. 57, no. 3, pp. 1675-1694, May 2008.

[81] X. Huang, J. Lu, J. Zheng, J. Chuang, and J. Gu, "Reduction of peak-to-average power ratio of OFDM signals with companding transform," Electron. Lett., vol. 37, no. 8, pp. 506-507, Apr. 2001.

[82] X. Huang, J. Lu, J. Zheng, K. B. Letaief, and J. Gu, "Companding transform for reduction in peak-to-average power ratio of OFDM signals," IEEE Trans. Wireless Commun., vol. 3, no. 6, pp. 2030-2039, Nov. 2004.

[83] T. Jiang and G. Zhu, "Nonlinear companding transform for reducing peak-toaverage power ratio of OFDM signals," IEEE Trans. Broadcast., vol. 50, no. 3, pp. 342-246, Sept. 2004 . 
[84] T. Jiang, W. Yao, P. Guo, Y. Song, and D. Qu, "Two novel nonlinear companding schemes with iterative receiver to reduce PAPR in multi-carrier modulation systems," IEEE Trans. Broadcast., vol. 52, no. 2, pp. 268-273, Jun. 2006.

[85] Y. Jiang, "New companding transform for PAPR reduction in OFDM," IEEE Commun.. Lett., vol. 14, no. 4, pp. 282-284, Apr. 2010.

[86] J. Davidson and I. Kalet, "Statistics of companding noise for QAM transmission," IEEE Trans. Commun., vol. 44, no. 3, pp. 306-311, Mar. 1996.

[87] J. X. Wang, T. T. Tjhung, and Y. Wu, "On the SER and spectral analyses of Alaw companded multicarrier modulation," IEEE Trans. Veh. Technol., vol. 52, no. 5, pp. 1408-1412, Sept. 2003.

[88] T. G. Pratt, N. Jones, L. Smee, and M. Torrey, "OFDM link performance with companding for PAPR reduction in the presence of non-linear amplification," IEEE Trans. Broadcast., vol. 52, no. 2, pp. 261-267, Jun. 2006.

[89] T. Jiang, W. Xiang, P. C. Richardson, D. Qu, and G. Zhu, "On the nonlinear companding transform for reduction in PAPR of MCM signals," IEEE Trans. Wireless Commun., vol. 6, no. 6, pp. 2017-2021, Jun. 2007.

[90] L. J. Cimini, Jr. and N. R. Sollenberger, "Peak-to-average power ratio reduction of an OFDM signal using partial transmit sequences," IEEE Commun. Lett., vol. 4, no. 3, pp. 86-88, Mar. 2000. 
[91] C. Tellambura and A. D. S. Jayalath, "PAR reduction of an OFDM signal using partial transmit sequences," in Proc. IEEE 54th Veh. Technol. Conf., pp. 465-469, Fall. 2001.

[92] Heung-Gyoon. Ryu, T. Hoa, K. Lee, S. Kim, and J. Park, "Improvement of power efficiency of HPA by the PAPR reduction and predistortion," IEEE Trans. Consumer Electron., vol. 50, no. 1, pp. 119-124, Feb. 2004.

[93] Yuh-Ren. Tsai and S. Huang, "PTS with Non-Uniform Phase Factors for PAPR Reduction in OFDM Systems," IEEE Commun. Lett., vol. 12, no. 1, pp. 20-22, Jan. 2008.

[94] C. Wang and Y. Ouyang, "Low-complexity selected mapping schemes for peakto-average power ratio reduction in OFDM systems," IEEE Trans. Signal Process., vol. 53, no. 12, pp. 4652-4660, Dec. 2005.

[95] J. Yang and L. Chen, "Reducing the Peak-to-average Power Ratio of OFDM Signal by a Modified Selected Mapping Technique," in Proc. 8th IEEE Intern. Conf. on Signal Process. ICSP 2006, vol. 3, 2006.

[96] G. T. Zhou and L. Peng, "Optimality condition for selected mapping in OFDM," IEEE Trans. Signal Process., vol. 54, no. 8, pp. 3159-3165, Aug. 2006. 
[97] Y. Jie, C. Lei, L. Quan, and C. De, "A modified selected mapping technique to reduce the peak-to-average power ratio of OFDM signal," IEEE Trans. Consumer Electron., vol. 53, no. 3, pp. 846-851, Aug. 2007.

[98] X. Yang, J. Wang, and D. Li, "Loosely synchronous code in selected mapping in OFDM," in Proc. Intern. Conf. on Wireless Commun., Networking and Mobile Computing WiCom 2007, Sept. 2007, pp. 141-144.

[99] B. K. Khoo, S. Y. Le Goff, C. C. Tsimenidis, and B. S. Sharif, "OFDM PAPR Reduction using selected mapping without side information," in Proc. IEEE Intern. Conf. on Commun. ICC'07, vol. 3, 2007, pp. 4341-4345.

[100] Yang Jie, C. Lei, L. Quan, and C. De, "A low complexity selected mapping scheme by use of time domain sequence superposition technique for PAPR reduction in OFDM system," IEEE Trans. Broadcast., vol. 54, no. 4, pp. 821824, Dec. 2008

[101] T. Helaly, R. Dansereau, and M. El-Tanany, “A low complexity PAR reduction technique using cyclic shifted data sequences in DS-CDMA signals," in Proc. 6th Adv. Intern. Conf. on Telecommun. AICT 2010, May 2010, pp. 1-5.

[102] P. E. Black, Greedy algorithm, in Dictionary of Algorithms and Data Structures, U.S. National Institute of Standards and Technology, Feb. 2005. 
[103] N. Hieu, Sang-Woo Kim, and Heung-Gyoon Ryu, "PAPR reduction of the low complexity phase weighting method in OFDM communication system," IEEE Trans. Consumer Electron., vol. 51, no. 3, pp. 776-782, Aug. 2005.

[104] N. Chen and G.T. Zhou, "Distortionless crest factor reduction for forward link CDMA," in Proc. 6th IEEE Workshop on Signal Process. in Wireless Commun, Jun. 2005, pp. 294-297.

[105] T. Helaly, R. Dansereau, and M. El-Tanany, "Factors affecting spectral regrowth in DS-CDMA signals due to PD-HPA nonlinear distortion," accepted in the IEEE 72nd Veh. Technol. Conf. VTC2010-Fall, Sept. 2010.

[106] T. Helaly, R. Dansereau, and M. El-Tanany, "Improved power amplifier efficiency via Walsh code assignment in synchronous CDMA systems," in Proc. 5th Adv. Intern. Conf. on Wireless and Mobile Commun. ICWMC 2009, Aug. 2009, pp. 179-184. 